\title{
The Role of BCL6 in Glioblastoma
}

\author{
By
}

Nicole Maree Jones

\begin{abstract}
A thesis
submitted to the Victoria University of Wellington in fulfilment of the requirements of the degree of Doctor of Philosophy
\end{abstract}

Victoria University of Wellington

2018 


\section{Abstract}

Glioblastoma (GBM) is the most common and most deadly brain tumour to occur in adults. Initially patients respond to radiation and chemotherapy, which primarily work by causing large amounts of DNA damage, leading to cell death. However, this process does not happen effectively in GBM and understanding how these cells resist cell death in response to therapy is key to improving the efficacy of treatment. BCL6 is a transcription factor that stops cell death in response to DNA damage, primarily through repressing transcription of DNA damage response genes. Recent work in our lab has shown BCL6 to be present in untreated GBM tumours and upregulated in GBM cells treated with chemotherapy or radiation, and inhibition of BCL6 leads to a profound loss in proliferative activity. These results indicate that BCL6 may be used as a mechanism of therapy resistance in GBM cells. The objective of this study was to establish a role for BCL6 in GBM cells using luciferase reporter assays, electrophoretic mobility shift assays (EMSA), quantitative chromatin immunoprecipitation (qChIP) and targeted inhibition of BCL6 with subsequent transcriptional analysis by RNA sequencing. We observed that BCL6 appeared to be a transcriptional activator in GBM, as shown by increased luciferase activity in GBM cells treated with radiation. EMSA experiments revealed that overexpressed BCL6 formed complexes with co-repressors, but endogenous BCL6 did not. qChIP experiments revealed that BCL6 was not bound to tradtional BCL6 target genes. Analysis of transcriptional profiles has identified a unique subset of genes which are downregulated when BCL6 is inhibited and upregulated in response to chemotherapy, and these genes were related to cell survival. These changes indicate that these genes may be regulated by BCL6 in chemotherapy treated cells. Together, these results illustrate that BCL6 appears to have an active and unique function in GBM cells, and reinforces this transcription factors position as an attractive therapeutic target in GBM. 


\section{Acknowledgements}

Well first I need to acknowledge my brilliant supervisor Dr. Melanie McConnell. Thank you for taking me on as your first PhD student, I hope I have done that honour justice. Thank you for believing in me, even when I didn't. You have given me every possible opportunity to further my personal and professional development throughout my time in your lab and I will be forever indebted to you for helping me to get where I am today. Thank you for your help, your encouragement and kindness, for pushing me, for making me a better scientist. I will never truly be able to thank you enough.

Mum and Dad, I would not be here without your never-ending love and support. It is impossible to express how grateful I am for everything you have done for me throughout my life, particularly in the last four years! Lance, I really appreciate your support over all these years I've been away from home.

I need to acknowledge all of my grandparents, who have always loved me and encouraged me to do my best, I hope I have made all of you proud. I must particularly acknowledge my Nan, whose untimely death is what inspired and drove me to study science, particularly to focus on cancer. This one is for you, and I'm so sorry that Grandad didn't quite get to see me finish it.

Everyone who has been in and is currently part of the MMc lab, you have put up with me, helped me, and supported me in ways you may not know. I have learned something from each and every one of you. I need to acknowledge a few members in particular:

Marie-Sophie Fabre, she pioneered this project and has been an invaluable resource for me, as well as a great cheerleader throughout my project.

Leticia Castro, who has helped raise me since I started in the lab, I hope I'm less of a pain than I once was!

Matthew Rowe, a long time friend and lab member who helped me with some experiments early on, and has always been great to talk to about my project, sorry for never remembering the details of yours. 
Sam Lee, who helped me so much with grappling with the RNA seq data, you made far too many sacrifices to help me out, your enthusiasm for your work will get you far in life.

Dini Senanayake, thank you for your input with my writing and your encouragement when I stayed late to write, you are a true gem.

My favourite women scientists, Amy Foster and Nikki Templeton, thank you so much for everything you have done for me over the years. You are both strong, incredibly talented women, who are destined for great things and I don't know what I would have done without your support.

AM304/312 crew thanks to everyone who I've shared an office/lab with over the past four years. We had lots of good times and I've learned a lot from being surrounded by people doing all sorts of different types of science.

MIMR PhD Club, particularly the dream team Alanna Cameron, Cam Field, Ryan Kyle, Cat Plunkett and Sou Ochiai. You all took me under your wing and taught me so much about science and managing life as a $\mathrm{PhD}$ student, I will be forever grateful for your help and advice.

My two best friends Emma Bagley and XinYa Zhou, thank you for keeping me sane and giving me a good excuse to get away from it all- it sucks not having you both here with me but it makes the visits all the more special. All of my other friends who are not in science (The Faves/Team Gold, Cult of Solon, Maccas crew, and many other individuals- you know who you are) thank you for listening to and sympathising with my rants about things you didn't quite understand, for reminding me that life exists outside of science and for always making me feel welcome and loved, I love you all!

Finally, my fiancé Cameron Stanton. I don't think either of us truly knew what I signed up for when I first started this $\mathrm{PhD}$, and I know that this would have been near impossible without you. I'm sorry for never giving you an accurate time I will be in the lab for (I'll just be 10 minutes, only to return 3 hours later.. oops!), spending so much time in the lab away from you, and being very emotionally 
invested in this thesis. You have been impossibly patient, kind, and understanding in the face of all that, and I truly appreciate it. 


\section{Table of Contents}

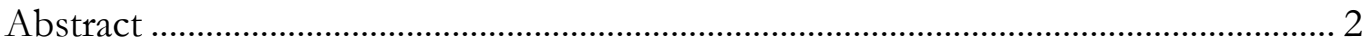

Acknowledgements.................................................................................................... 3

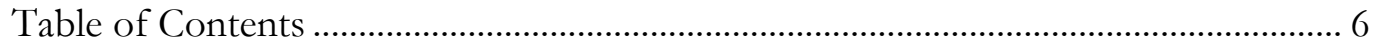

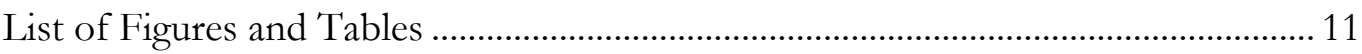

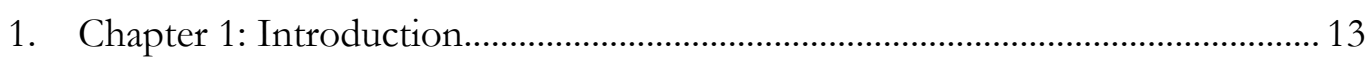

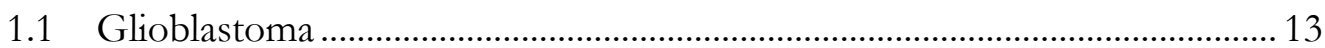

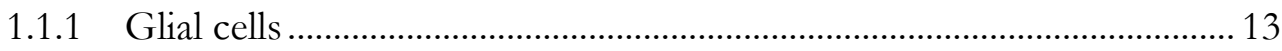

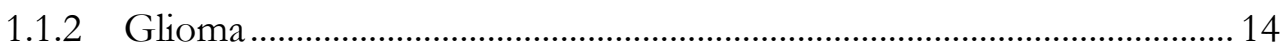

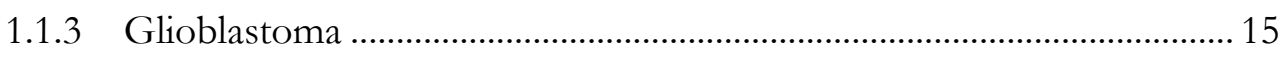

1.1.4 Molecular Features/Subtypes of GBM …………………........................ 16

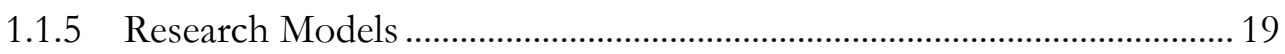

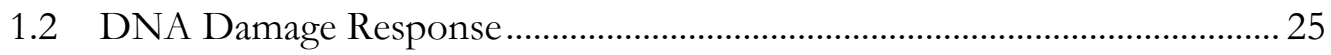

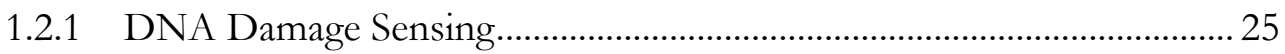

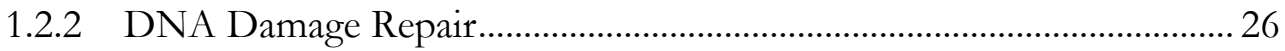

1.2.3 Apoptosis in Response to DNA Damage: The Mechanism of

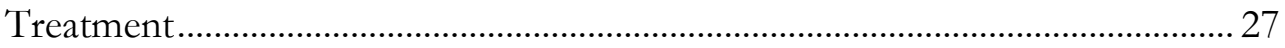

1.2.4 DNA Damage and Therapy Resistance in Glioblastoma....................... 30

1.2.5 Other Therapeutic Approaches ................................................................... 32

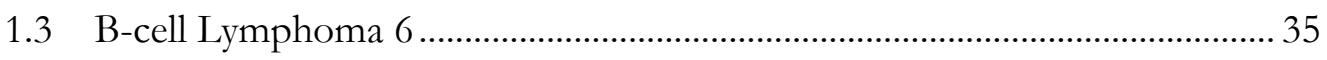

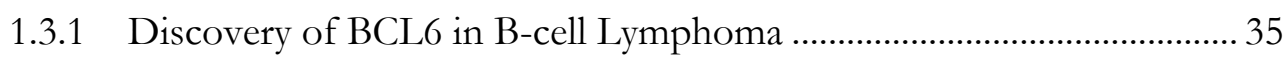

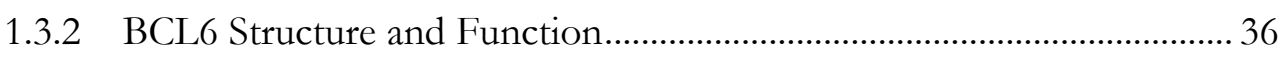

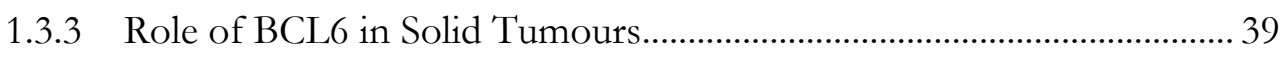

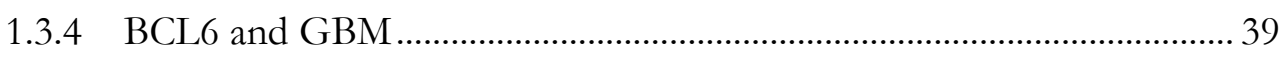

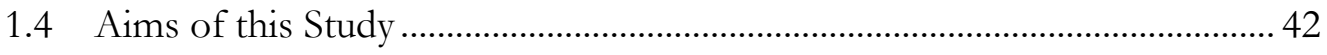

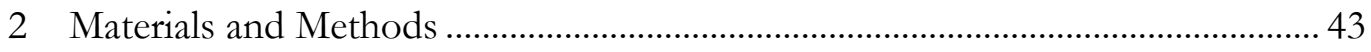

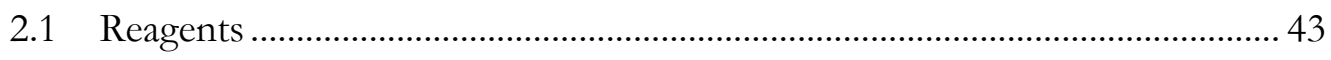

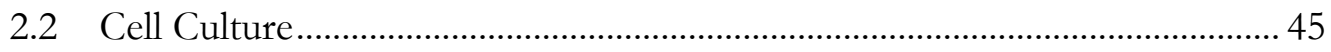

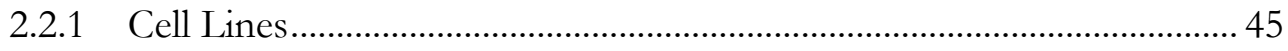

2.2.2 Cell Culture Media ............................................................................ 45

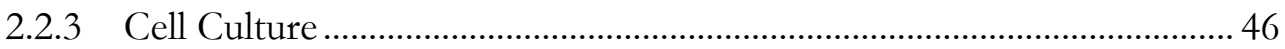

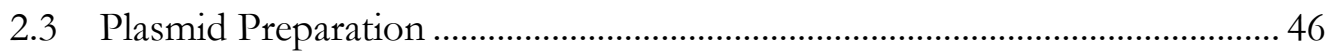


2.3.1 Bacterial Transformation and Culture

2.3.2 Plasmid Preparation and DNA Precipitation ............................................. 47

2.4 DNA, RNA and Protein Quantification............................................................. 48

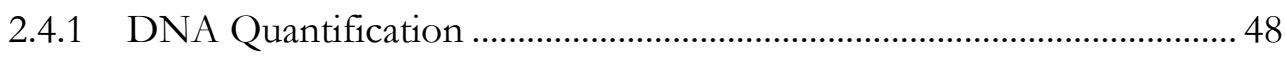

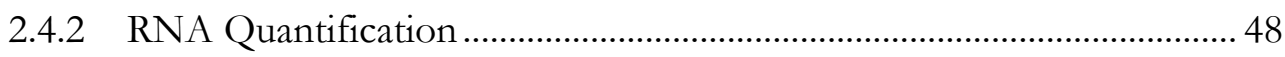

2.4.3 Protein Quantification .............................................................................. 49

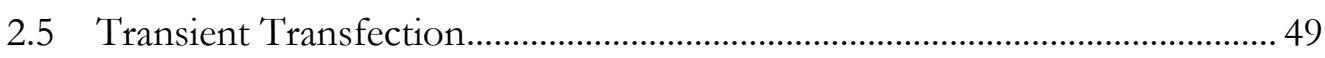

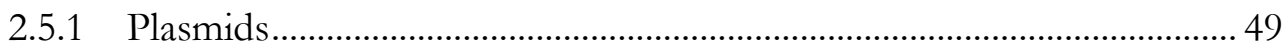

2.5.2 LTX and Plus Transfection..................................................................... 49

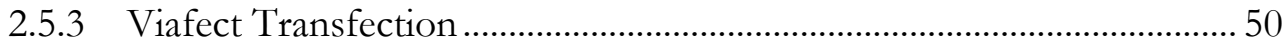

2.6 Luciferase Reporter Assay ................................................................................. 50

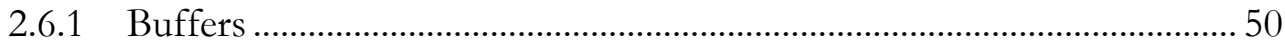

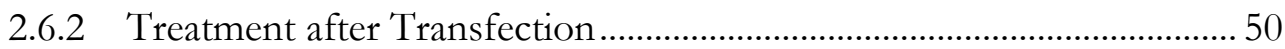

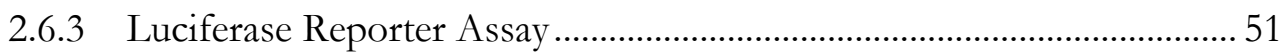

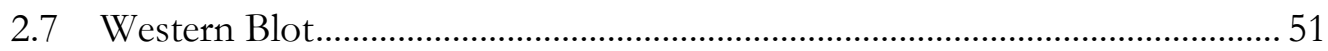

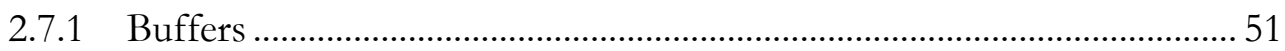

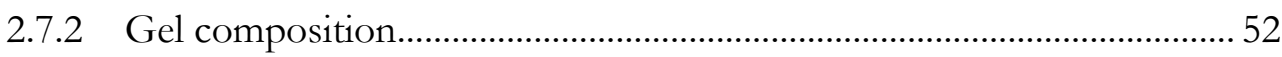

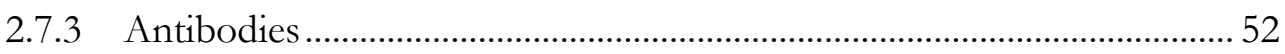

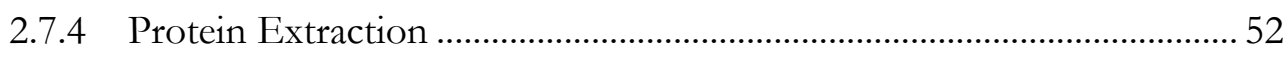

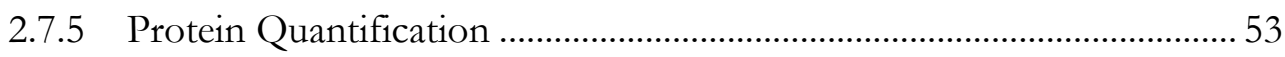

2.7.6 SDS-Acrylamide Gel Preparation................................................................... 53

2.7.7 SDS- Polyacrylamide Gel Electrophoresis................................................... 53

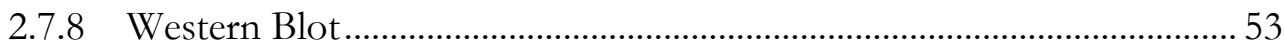

2.8 Electrophoretic Mobility Shift Assay ............................................................... 55

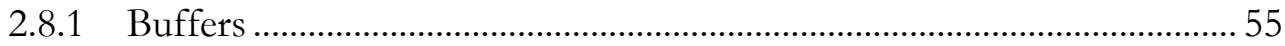

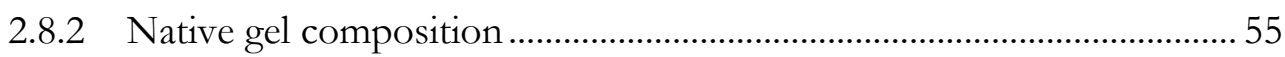

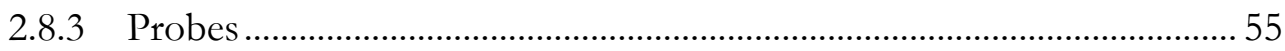

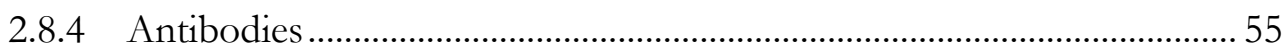

2.8.5 Cell preparation for EMSA …………………....................................... 56

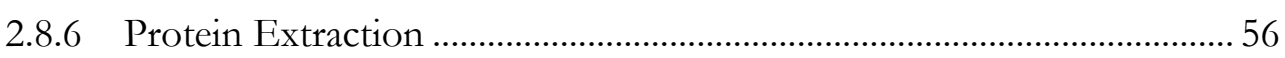

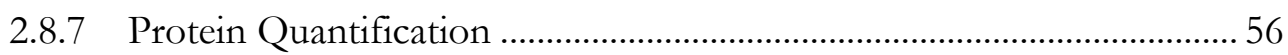

2.8.8 Native Acrylamide Gel Preparation.......................................................... 56

2.8.9 Labelled Probe and Unlabelled Competitor DNA Preparation............. 57

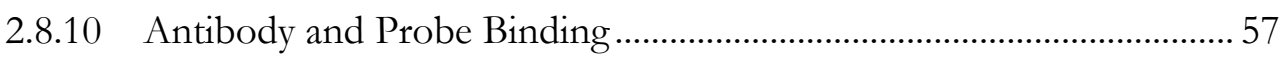


2.8.11 Gel Electrophoresis and Transfer

2.8.12 Cross-linking and Detection of Biotin-labeled DNA …........................58

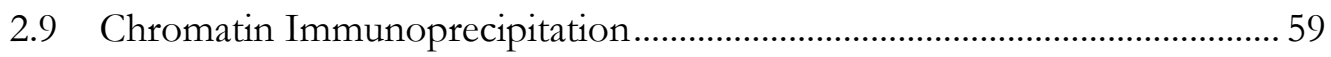

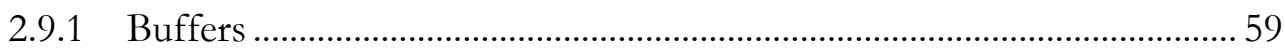

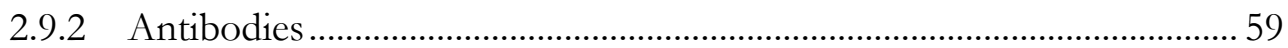

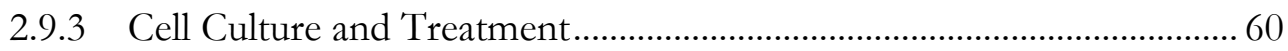

2.9.4 Agarose Bead Preparation (Melnick Lab) ………………………….......... 60

2.9.5 Dynabead $®$ Preparation (McConnell Lab)...............................................60

2.9.6 Cell Fixation, Harvest and Nuclear Preparation ........................................ 61

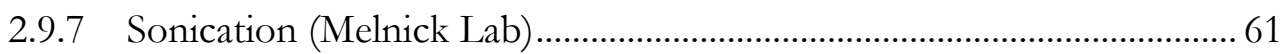

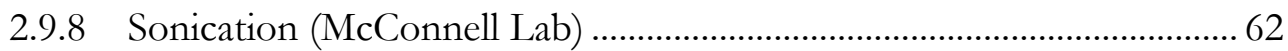

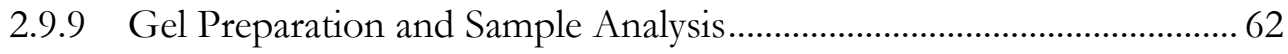

2.9.10 Preclearing and Immunoprecipitation (Melnick Lab) ............................ 62

2.9.11 Immunoprecipitation (McConnell Lab) .................................................... 63

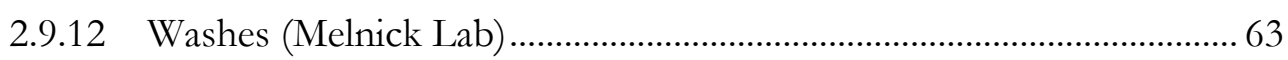

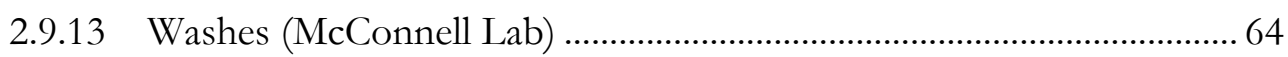

2.9.14 Reverse Cross-links and DNA Extraction.............................................. 64

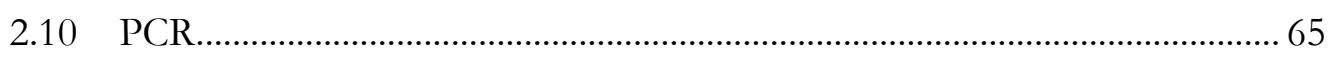

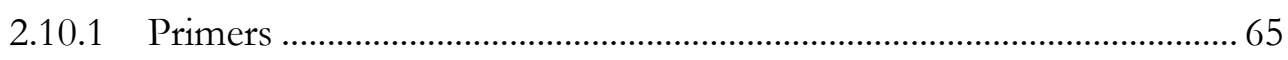

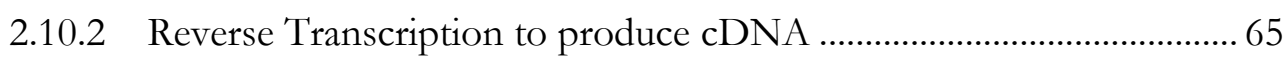

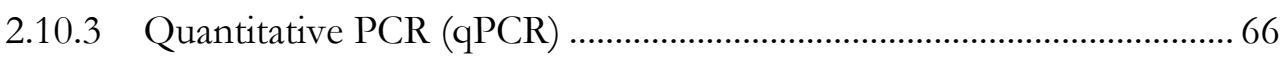

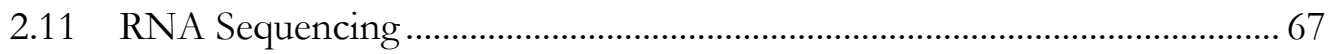

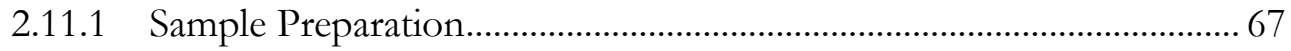

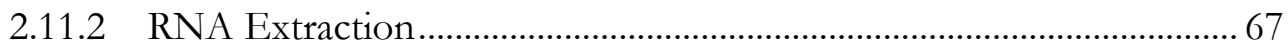

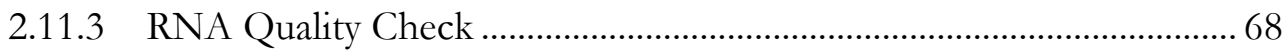

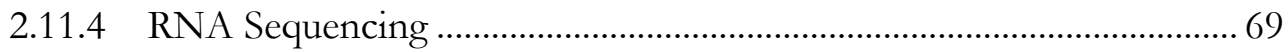

2.11.5 RNA Sequencing Data Processing............................................................. 69

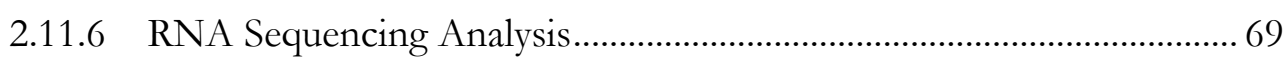

3 Expression and Activity of BCL6 in Glioblastoma …………………….................. 71

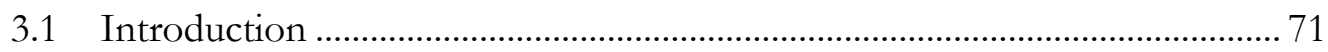

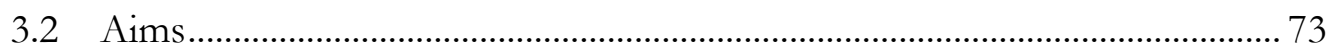

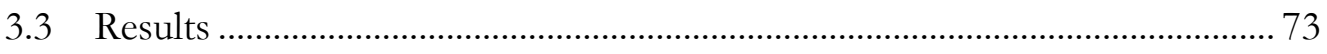

3.3.1 Expression of BCL6 and co-repressors in glioblastoma cells LN18 and

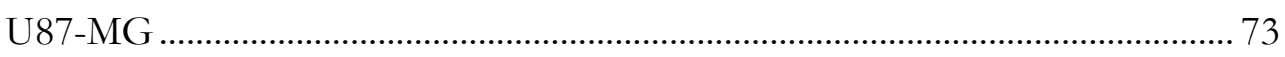


3.3.2 Transcriptional Activity of BCL6 in LN18 cells.

3.3.3 Transcriptional Activity of BCL6 in U87-MG cells ................................ 95

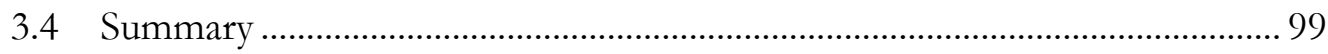

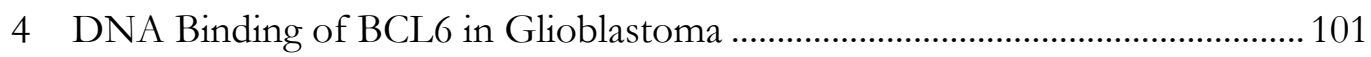

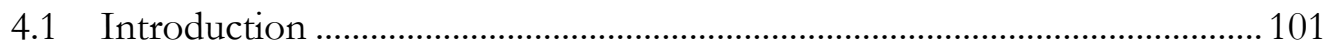

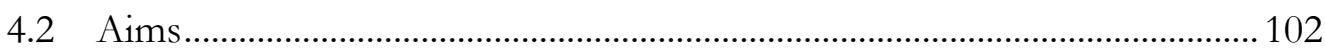

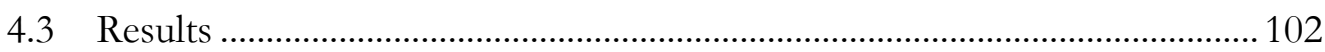

4.3.1 BCL6 interaction with DNA binding motif ........................................ 102

4.3.2 Establishment of chromatin immunoprecipitation in LN18 ............... 108

4.3.3 qChIP on Doxorubicin treated LN18 cells .............................................. 110

4.3.4 qChIP on Radiation treated LN18 cells .................................................... 116

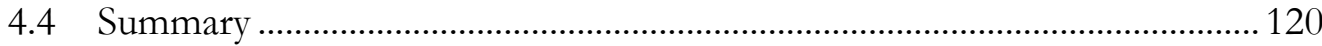

5 Effect of BCL6 on Gene Expression in Glioblastoma......................................... 123

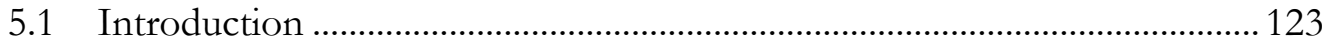

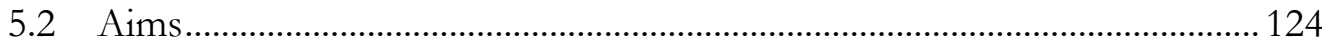

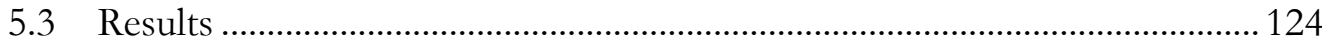

5.3.1 Planning and preparation of RNA samples for sequencing.................. 124

5.3.2 Quality of RNA Sequencing..................................................................... 129

5.3.3 Principal Component Analysis .............................................................. 130

5.3.4 Differential Expression Analysis .......................................................... 133

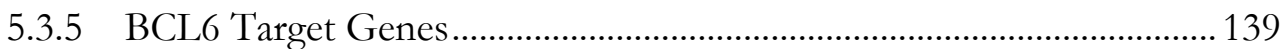

5.3.6 Gene Set Enrichment Analysis .................................................................. 142

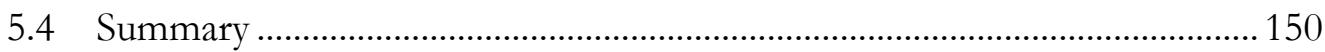

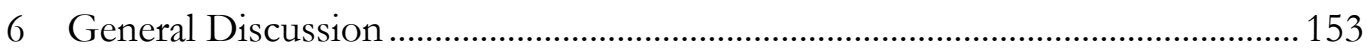

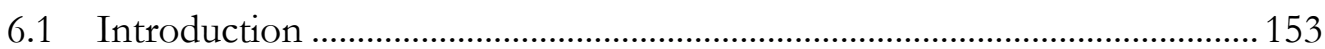

6.2 Determine that BCL6 is present and transcriptionally active in GBM cells 154

6.2.1 BCL6 is transcriptionally active in GBM cells, but it behaves differently

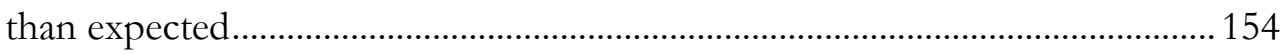

6.2.2 Choice of chemotherapy .......................................................................... 155

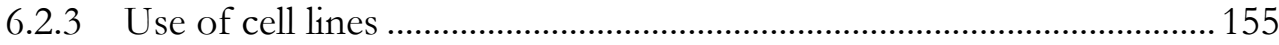

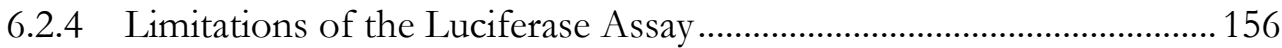

6.3 Investigate BCL6 binding to DNA and BCL6 target genes in GBM cells 157

6.3.1 Endogenous BCL6 did not bind its consensus sequence in LN18 .... 157 
6.3.2 Endogenous BCL6 does act as a transcriptional repressor at the TARS locus 157

6.3.3 Variation in chromatin immunoprecipitation protocols 158

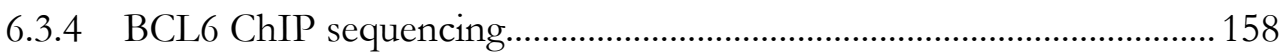

6.4 Investigate the effect of BCL6 inhibition on global transcription in GBM cells 159

6.4.1 BCL6 inhibition had no effect on AXL kinase 159

6.4.2 BCL6 inhibition had a profound effect on gene expression. 160

6.4.3 Limited relationship between BCL6 inhibition and ionising radiation 160

6.5 Future Directions 162

6.6 Summary 162

7 References 163

8 Appendix 183 


\section{List of Figures and Tables}

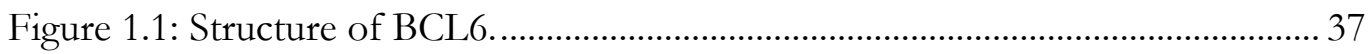

Figure 3.1: q-RT-PCR shows expression of BCL6 and co-repressors BCOR, NCOR, and SMRT in LN18 cells. ......................................................................... 76

Figure 3.2: q-RT-PCR shows expression of BCL6 and co-repressors BCOR,

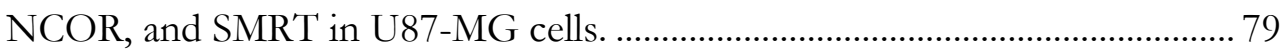

Figure 3.3: BCL6 is up-regulated in response to doxorubicin in LN18 cells. ........... 81

Figure 3.4: BCL6 is not up-regulated in response to treatment in U87-MG cells.... 82

Figure 3.5: The co-repressors BCOR, NCOR, and SMRT are present in LN18 cells.

Figure 3.6: The co-repressors BCOR, NCOR, and SMRT are present in U87-MG cells.

Figure 3.7: Effect of transfection of LN18 with LTX and Plus versus Viafect........ 88

Figure 3.8: Overexpressed BCL6 is a transcriptional repressor in LN18 cells.......... 90

Figure 3.9: Endogenous BCL6 is not transcriptionally active in LN18 cells............. 91

Figure 3.10: Therapy induced BCL6 is not a transcriptional repressor in LN18 cells.

Figure 3.11: Radiation treatment leads to an increase in BCL6 $6_{4}$ tkLUC activity...... 94

Figure 3.12: Effect of transfection on U87-MG cells with LTX and Plus versus Viafect 96

Figure 3.13: Overexpressed BCL6 is not a transcriptional repressor in U87-MG cells. 98

Figure 4.1: Establishment of Electrophoretic Mobility Shift Assay (EMSA) with control from kit.

Figure 4.2: Overexpressed BCL6 forms distinct complexes with co-repressors.... 106

Figure 4.3: Natively expressed BCL6 does not form complexes with BCL6 binding consensus sequence.

Figure 4.4: Optimisation of sonication protocol for Chromatin Immunoprecipitation (ChIP). 109

Figure 4.5: Establishment of BCL6 ChIP assay in Melnick Lab at Weill Cornell Medical College, New York City.

Figure 4.6: BCL6 and RNA Polymerase II ChIP on doxorubicin treated cells shows reproducible positive and negative controls. 
Figure 4.7: Binding of BCL6 at BCL6 Target Genes does not significantly change with doxorubicin treatment.

Figure 4.8: BCL6 and RNA Polymerase II ChIP on radiation treated cells shows reproducible positive and negative controls.

Figure 4.9: Binding of BCL6 at BCL6 Target Genes does not significantly change with radiation treatment.

Figure 5.1 Pictures of LN18 cells used for RNA sequencing just before harvest for RNA 128

Figure 5.2: Principal Component Analysis of the RNA sequencing data-set. 132

Figure 5.3 Volcano plots of differential expression 135

Figure 5.4: Significant differentially expressed genes between data-sets. (A) 138

Figure 5.5: Heatmap of BCL6 target gene expression in RNA seq data-sets. 141

Figure 5.6: Significant GSEA Hallmarks Data-sets. 148

Figure 5.7: Up- and Down-regulated GSEA Hallmarks data-sets. 149

Table 3.1: Average Ct values for BCL6 and co-repressors in LN18 cells 75

Table 3.2: Average Ct values for BCL6 and co-repressors in U87-MG cells............ 78

Table 5.1 RNA quality of samples used for sequencing. 127 


\section{Introduction}

\subsection{Glioblastoma}

There was once a time where the word "cancer" was only uttered in hushed tones behind closed doors. It was known that the "big C", in any form, was a death sentence. With advances in science and medicine, cancer is now less frequently a death sentence and some types are curable or are more of a chronic disease. Unfortunately, this has not happened for all types of cancer- a diagnosis of glioblastoma is still very much a death sentence.

\subsubsection{Glial cells}

The term neuroglia (ancient Greek for 'nerve glue'), originally coined by Rudolph Virchow in 1856, was used to describe what was thought to be extracellular matrix that supported neurons in the brain (1). It was later discovered that this extracellular matrix was in fact made up of many types of cells that are important for supporting neurons and thus the term neuroglia (and also the shortened term glia) has since been used to describe all of these cells.

Glial cells are derived from neural stem cells (NSCs), which are present in both the embryonic and adult brain. Glial cells can be further divided into two broad categories: oligodendrocytes and astrocytes. Oligodendrocytes are responsible for the formation of the myelin sheath on nerve fibres in the brain (2), and are derived from oligodendrocyte precursor cells (OPCs), which are derived from NSCs (3). OPCs are found in the adult brain and continue to divide into adulthood. Astrocytes provide broad homeostatic control of neurons, which maintains neuron health (4). A physiological precursor cell for astrocytes has not yet been identified, but astrocytes themselves are known to have proliferative capacity (5). Glioma is cancer in the brain that primarily involves glial cells and subsequently, NSCs, OPCs and astrocytes all have the potential to be cells of origin for glioma. 


\subsubsection{Glioma}

Glioma is a broad term that refers to all tumours in the brain that appear to be of glial origin. Glioma can occur in both adults and children, and childhood disease is different from adult disease. For the purpose of this thesis, the focus will be on adult glioma., and thus when the term "glioma" is used, it is referring to adult glioma.

Gliomas are graded from I-IV, with II-III classed as lower grade gliomas (LGGs) and IV classed as high-grade glioma (6,7). Grade I gliomas are distinct from all other gliomas; they are benign tumours with well-defined margins, which are curable by surgical resection. Lower grade gliomas are infrequent tumours, accounting for around 15\% of all gliomas, and are found in a younger population, with peak incidence between 35 and 44 years old $(8,9)$. LGGs are a collection of diffuse gliomas and are defined as either astrocytoma, oligodendroglioma, or oligoastrocytoma based on histology (7). The most recent study for the treatment of LGG showed that LGGs have a median overall survival time of 13.3 years when treated with surgical resection of the tumour followed by fractionated radiation therapy (30 fractions of $1.8 \mathrm{~Gy}$ ) and chemotherapy, which involves six 8-week cycles of procarbazine $\left(60 \mathrm{mg} / \mathrm{m}^{2}\right)$, CCNU $\left(110 \mathrm{mg} / \mathrm{m}^{2}\right)$ and vincristine (1.4 $\left.\mathrm{mg} / \mathrm{m}^{2}\right)(10)$. This is likely to become the standard of care for LGGs in the near future as this regime has a significant survival benefit over radiation treatment alone (7.8 years with radiation alone versus 13.3 years with combined treatment).

In 2016 there was an update of the World Health Organisation (WHO) classification of tumours of the CNS, which incorporated molecular information as part of the classification of gliomas and has lead to a profound reshuffling of the classification of LGGs (7). LGGs can be divided into three distinct molecular subtypes- isocitrate dehydrogenase $1 / 2$ (IDH) mutation with co-deletion of chromosome arms 1p/19q, IDH mutation without co-deletion of $1 \mathrm{p} / 19 \mathrm{q}$ and $I D H$ wild-type (6). LGGs with $I D H$ mutation and $1 \mathrm{p} / 19 \mathrm{q}$ deletion have the best survival (median 8.1 years), IDH mutation and no $1 \mathrm{p} / 19 \mathrm{q}$ deletion have relatively good survival (median 6.3 years), and IDH wild-type tumours have the worst survival, 
with a median of 1.7 years, which is more similar to the prognosis for grade IV gliomas (6).

Enzymes IDH1 and IDH2 catalyse the decarboxylation of isocitrate to $\alpha$ ketoglutarate $(\alpha-K G)$ alongside the reduction of cofactor nicotinamide adenine dinucleotide phosphate $\left(\mathrm{NADP}^{+}\right)$to $\mathrm{NADPH}$, and also catalyse the reverse reaction, depending on cellular conditions $(11,12)$. IDH1 performs this in the cytoplasm and IDH2 performs this in the mitochondria. This reaction is important primarily for the reduction of reactive oxygen species (ROS) through multiple mechanisms (13). IDH1 and 2 can be mutated in their active site, the most common mutation being $I D H 1^{\mathrm{R} 132 \mathrm{H}}$, which causes an incomplete reverse reaction to occur, leading to the production of a new molecule, 2-hydroxyglutarate (2-HG) instead of isocitrate (14). $2-\mathrm{HG}$ is a competitive inhibitor of $\alpha-K G$ dependent histone demethylases and TET 5-methylcytosine hydroxylases. As a result, high amounts of 2-HG, such as those seen in IDH mutant gliomas, lead to altered histone and DNA methylation (15). This leads to a hypermethylated state in glioma called G-CIMP (glioma-CpG island methylator phenotype) (16,17). G-CIMP is when CpG islands along DNA have more methylation than what would be considered normal (hypermethylated), and when this occurs at a gene promoter, it prevents transcription of the gene. This has been shown to be important in both LGGs and high-grade gliomas.

\subsubsection{Glioblastoma}

Glioblastoma (GBM) is the most common and most deadly form of brain tumour to occur in adults. GBM is a grade IV diffuse glioma, which means it is highly invasive and grows very quickly, resulting in a highly disorganised arrangement of tumour cells, blood vessels and other cells involved in the tumour microenvironment. GBM occurs in two forms, primary and secondary GBM. Primary GBM is diagnosed without evidence of a previous low grade tumour, is IDH1 wild type, and is the most common form of GBM, accounting for more than $90 \%$ of cases $(18,19)$. Secondary GBM develops after the diagnosis of a lower grade glioma, and is usually IDH1 mutant. Primary GBM and secondary GBM affect distinctly different populations of people. Primary GBM commonly occurs in older 
people, with an average age of 62 years and secondary GBM usually occurs in younger people with an average age of 45 years $(18,19)$.

The prognosis for patients diagnosed with GBM is often described as 'dismal' with a median overall survival of 19 months (with the most extensive surgical removal, followed by the most aggressive therapy regime) after diagnosis with current treatment and a 5 year survival rate of less than 10\% (20). The standard treatment currently used to treat people with GBM is known as the "Stupp Protocol" after Dr. Roger Stupp, the first author of the clinical trials that lead to the use of temozolomide (TMZ). The Stupp Protocol consists of complete surgical resection of the tumour, followed by concomitant ionising radiation (IR) (60 Gy total, 2 Gy daily Monday- Friday) and TMZ (75 mg/m² daily) followed by adjuvant TMZ (6 cycles consisting of $150-200 \mathrm{mg} / \mathrm{m}^{2}$ for 5 days during each 28 -day cycle) (20). The effectiveness of chemo-radiation is limited as the tumour will inevitably relapse, with median time to relapse 6.9 months with most extensive surgical resection (20). When the tumour relapses the patient can choose to have further adjuvant TMZ or other interventions, including further surgical resection, which may offer benefit for some patients. Ultimately the decision for further treatment comes down to the patient, as all treatments have side-effects and may not give them the quality of life they want in their final moments.

\subsubsection{Molecular Features/Subtypes of GBM}

Currently there are few known molecular features that make any clear difference to the clinical outcome of GBM. One of these is MGMT $\left(\mathrm{O}^{6}\right.$-methylguanine-DNA methyltransferase) promoter methylation. The function of MGMT is to repair $\mathrm{O}^{6}$ methylguanine lesions caused by TMZ by removing the lesion from the base, which reduces the efficacy of TMZ treatment substantially. Patients with MGMT promoter methylation have a significant survival benefit (median survival 23.4 months with MGMT methylation vs 12.6 months without), as the MGMT promoter is inactive, which means there is no transcription of MGMT and subsequently no active MGMT to prevent the action of TMZ (20). 
The other molecular feature of GBM that makes a difference to clinical outcome is IDH mutations, though it is not yet clear the exact mechanism by which this works. Less than $5 \%$ of primary GBMs and $>80 \%$ of secondary GBMS are IDH mutant, which is in line with secondary GBMs being a tumour that originated as a LGG, as LGGs have a high rate of IDH mutation $(18,21)$. While in LGGs IDH mutation and G-CIMP methylation tend to go hand in hand, IDH mutant GBMs have both GCIMP high and G-CIMP low groups, with G-CIMP high tumours having a more favourable prognosis (median survival 34.5 months) than G-CIMP low tumours (median survival 9.7 months) (16,22). This survival benefit of IDH mutant, GCIMP high tumours has been associated with MGMT methylation status (23), although this association is not consistent across different GBMs (24).

GBM is a heterogeneous tumour, which means that instead of containing cells that have all of the same mutations, it contains a variety of cells with different mutations, allowing it to adapt and grow regardless of its environment (25). This has made it complicated to describe GBM at a molecular level as many molecular entities exist within the same tumour, and sampling and subsequent molecular typing of the tumour may not accurately reflect this heterogeneity. Over the past decade, the cancer genome atlas (TCGA) has addressed this problem, using large cohorts of patient samples and a variety of molecular techniques (6,22,26-28). Subsequently they have made great progress into getting a complete molecular picture of GBM, which should prove to be useful in the development of new therapeutics.

In 2010, Verhaak et al. defined 4 distinct molecular subtypes of glioblastoma: classical, mesenchymal, proneural and neural (26). The classical subtype was characterised by epidermal growth factor receptor (EGFR) gene amplification, which occurred in $97 \%$ of classical tumours and homozygous deletion of $9 \mathrm{p} 2.13$, which targeted CDKN2A (cyclin-dependent kinase inhibitor 2A) and co-occurred with EGFR amplification $94 \%$ of the time (26). The mesenchymal subtype was characterised by deletions of neurofibromin 1 (NF1), which primarily co-occurred with phosphatase and tensin homolog (PTEN) loss, and was generally more necrotic and inflammatory than other subtypes of tumours as demonstrated by high expression of tumour necrosis factor superfamily members RELB proto-oncogene NF-KB subunit (RELB), TNFRSF1A associated via death domain (TRADD) and 
TNF receptor superfamily member $1 \mathrm{~A}$ (TNFRSF1A) (26). The proneural subtype was characterised by either amplifications in platelet derived growth factor receptor a (PDGFRA) or mutations in IDH1, and the IDH1 mutant subtype could be further split based on G-CIMP status (26). The neural subtype was characterised by expression of neural markers, for example neurofilament light (NEFL), gammaaminobutyric acid type A receptor alpha1 subunit $(G A B R A 1)$ and Synaptotagmin 1 (SYT1), which are normally expressed in differentiated neurons.

In 2013, the follow up paper was published by Brennan et al. which further broke down these 4 GBM subtypes into 5: proneural G-CIMP, proneural non G-CIMP, neural, classical, and mesenchymal (28). This paper also found that there were 6 methylation subtypes of GBM: M1, M2, M3, M4, G-CIMP, and M6. Clusters M1 and 2 were enriched for mesenchymal GBMs, M3 and 4 for classical GBMs, and the G-CIMP and M6 clusters are enriched for proneural GBMs. The proneural non GCIMP group had significant association with PDGFR $A$ amplification, and the proneural G-CIMP group had enrichment for ATRX mutations and MYC amplifications (28).

Brennan et al. also gave a comprehensive view of the somatic mutations in glioblastoma, highlighting mutations in at least one receptor tyrosine kinase (RTK) to be common (67.3\% of tumours analysed): either EGFR (57.4\%), PDGFRA (13.1\%), MET proto-oncogene, receptor tyrosine kinase (MET) (1.6\%), or fibroblast growth factor receptor 2/3 (FGFR2/3) (3.2\%). Mutations affecting the phosphatidylinositol-3-kinase (PI3K) pathway were common, affecting 59.4\% of tumours overall. Mutations in PI3K family members (particularly p110alpha and p85alpha subunits) were found in $25.1 \%$ of tumours, and PTEN mutations/deletions were mutually exclusive from PI3K mutations. When including the RTK mutations, $89.6 \%$ of all tumours analysed had at least one alteration in the PI3K pathway. The P53 pathway was dysregulated in $85.3 \%$ of tumours through mutations/deletion of TP53, amplification of MDM1/2/3 proto-oncogene (MDM1/2/3), or deletion of cyclin dependent kinase inhibitor 2A (CDKN2A).

Since the Verhaak et al. and Brennan et al. publications, further classification of a range of diffuse gliomas (grade II-IV) has been completed by Ceccarelli et al., which 
has lead to more accurate groups of tumours, which has allowed for more accurate predictions of treatment outcome(22). This strengthens the relationship across diffuse gliomas; even though a histological distinction can be made between low and high-grade tumours, having a clear molecular definition of gliomas gives a better picture of the clinical outcome of any individual tumour.

Ceccarelli et al. looked at a range of tumours (grade II-IV) and found that they can first be split into IDH mutant or wild type, which generally splits the lower grade (IDH mutant) from the high grade (IDH wild type) tumours. The IDH mutant group splits into three different subtypes. The first split is based on G-CIMP status $(16,29)$. As discussed earlier, people whose tumours have low G-CIMP have lower survival rates, while G-CIMP-high tumours have higher survival. The third subtype are those tumours with co-deletion of $1 \mathrm{p}$ and $19 \mathrm{q}$, which have the highest survival of all defined subtypes (median survival 9.7 years). The IDH1 wild type tumours split into 4 subtypes. The classic-like and mesenchymal-like subtypes both have low survival as defined in $(26,28)$. The LGm6-GBM subtype, which is defined by methylation subtype, and Pliocytic astrocytomalike (PA-like), both have higher survival than the classic-like and mesenchymal-like subtypes, with LGm6-GBM having lower survival than PA-like (22).

These studies have highlighted the complexity of gliomas and shown that grade does not necessarily determine the molecular phenotype or outcome of treatment. Complete molecular subtyping of GBM tumours is not used in the clinic, as it does not yet give clinicians useful information with regards to treatment for their patients. Despite a large amount of research in this area, it is not yet clear whether these molecular subtypes will provide useful information for the future treatment of GBM.

\subsubsection{Research Models}

GBM is an incredibly complex disease, with high inter- and intra-tumoural heterogeneity. Researchers face a huge challenge in the development of models that accurately recapitulate disease. As a result, many approaches have been used to model GBM, and over time as more information becomes available about GBM 
tumours these models get closer to fully reflecting the disease. A wide variety of GBM models are used by researchers, as not all models are appropriate to answer the kinds of questions that researchers are asking and some are much more expensive and technically challenging than others. In this thesis the well established cell lines LN18 (30) and U87-MG (31) have been used, which are derived from human tumours and have been used to model GBM in vitro for many years.

The cell line LN18 was established in 1981 by Diserens et al. from a tumour that had been surgically removed from a 61 year old male with a right malignant glioma (30). The cells were grown easily in Dulbecco's Modified Eagle Medium supplemented with foetal calf serum and were able to be serially transplanted subcutaneously in nude mice. LN18 has been characterised as TP53 mutant and PTEN wild-type, and has been classed as the mesenchymal subtype of GBM (32) (Dr. Jason Huse, personal communication). LN18 is also classified as TMZ resistant (33). Since the establishment of this cell line it has been used extensively for research into glioblastoma, primarily as a cell line grown in culture.

U87-MG is a popular cell line as it can be easily cultured in vitro and also grown both intracranially and subcutaneously in immunodeficient mice. U87-MG was first established by Pontén and Macintyre in 1968 (31) and has been used extensively for research into glioblastoma in the years following. U87-MG is TP53 wild-type and PTEN null and sensitive to TMZ (32,33). Recently, members of the lab where U87MG originated conducted an investigation into the U87-MG line that is held by the ATCC and compared it to the original tumour material. The investigation found that the ATCC U87-MG cell line did not match the tumour material (34). They did establish that the widely used U87-MG is likely a cell line of brain tumour origin, but were unable to determine what type of tumour the cells came from. Subsequently, publications that have used the model must be interpreted with caution, as it is unknown whether U87-MG is an accurate reflection of glioblastoma.

GBM cell lines that have been maintained in vitro for long periods of time are unlikely to fully encompass all the facets of the disease itself, and it has been known for a long time that these cells are changed by the in vitro environment (35). It has 
been shown that GBM cells growing in vitro have a vastly changed transcriptional profile, which reflects the fact that the environment cells are grown in in vitro does not reflect the in vivo environment of the tumour (36). However, glioblastoma cell lines are reproducible, relatively easy and cheap to cultivate, and they provide a starting point for research into glioblastoma before moving into in vivo models.

To address the issues with models of GBM that are primarily in vitro, multiple in vivo models of GBM have been developed, which usually involve tumour cells being implanted into the brains of mice. There are multiple ways of setting up this kind of model, most commonly using tumour material from a patient into an immunocompromised mouse or implanting a mouse tumour cell line into the same type of mouse.

A popular model of GBM is to take patient tumour material and propagate it in specialised stem cell media, which leads to the selection of glioma stem cells (GSCs). GSCs are highly infiltrative cells that repopulate the tumour after treatment. These cells are inherently therapy resistant, express high levels of neural stem cell markers and also tend to occur at infiltrating margin of GBMs, which makes it unlikely they will be removed during surgical resection (37-40). GSCs that are grown from primary tumour material in vitro have been shown to recapitulate the tumours they have come from when implanted in immunocompromised mice (4143). As a result, GSCs have become a popular in vitro and in vivo model of GBM. GSCs can be studied extensively in vitro before moving to in vivo work, reducing the number of animals required for this research.

A key problem with stem cell cultures is that it is a selective process and subsequently not all parts of the tumour are represented in the model. As a way to get around this, patient derived xenografts (PDX) were developed, where tumour material from a patient is directly grown in immunocompromised mice (either nude or NOD/SCID) (44). Initially the tumour is grown heterotopically in the flank of the mouse and tumours are serially passaged between mice to establish a long-term model (45). Once the tumours are established heterotopically they can be grown orthotopically. PDX models of GBM recapitulate the histology and molecular profile of their parent tumours, and have also been shown to reflect patient 
response to treatment $(44,46)$. However, a key problem with both stem cell and PDX models of GBM is that they need to be implanted into immunocompromised mice, which completely removes the interaction between the tumour and the immune system.

One way to get around the problem of using immunocompromised mice is to develop a tumour within the mouse itself so that the immune system stays intact. This approach was one of the earliest ways of modelling GBM (and many other tumours). One of the most established mouse cell lines used to model GBM is Glioma 261 (GL261), which was originally developed in 1939 by intracranial injection of the carcinogen 3-methylcholanthrene into C57BL/6 mice, and maintained by serial implantation of tumour fragments into syngeneic mice $(47,48)$. By the mid 1990s a cell line had been established from this tumour in multiple laboratories, primarily for the development of immunotherapies (49-51). GL261 cells grow easily in vitro and produce an aggressive tumour that mice succumb to quickly. The GL261 tumour does not fully recapitulate all of the characteristics of human GBM, as GL261 cells do not have a similar molecular profile to GBM, and are mildly immunogenic; vaccination with irradiated GL261 cells provides some level of protection against these tumours (52). Despite this, GL261 is still a popular mouse model of GBM, particularly when studying interactions between the immune system and the tumour.

To try and address the problems presented by the GL261 mouse model while keeping the immune system intact, genetically engineered mouse models (GEMMs) have been developed. GEMMs of GBM that recapitulate the disease well are difficult to develop, as it is not yet clear the order of mutational events that lead to GBM development, and mice will be different to humans in the way they develop disease. Researchers subsequently have to choose what the driver mutations are likely to be based on current molecular information. As a result, there are many different genetically engineered models of GBM, all with slightly different characteristics.

The first GEMM to be developed for brain tumours was by Brinster et al., where SV-40 T-antigen plasmids were microinjected into fertilised mouse eggs, which lead 
to reproducible development of tumours in the choroid plexus (53). Initially, most models were developed like this through germline mutations, which modelled familial predisposition to disease. Since then, many different, more complex models have been developed to target brain specific cells, all with varying success at producing truly "GBM-like" tumours (54-56). These models use modifications of pathways commonly mutated in GBM in different combinations, for example EGFR, PDGFR, P53 and PTEN. One of the earliest models of this is the v-src model, where v-src is targeted to glial fibrillary acidic protein (GFAP) expressing cells, which are primarily astrocytes. V-src interacts with EGFR and PDGFR, which are commonly upregulated in GBM, and expression of v-src in GFAP expressing cells results in the development of astrocytoma-like tumours (57). As technology progressed in this area, more techniques to target genetic changes in somatic cells in specific organs became available like tv-a/RCAS viral vectors, flox/cre, and most recently the CRISPR/Cas9 system (58-60). CRISPR/Cas9 is new technology that is relatively easy to use to target specific sites of the genome for modification, and will likely lead the way for the development of new GEMMs in the future. An example of this is use of the CRISPR/Cas9 system to target and delete TP53, NF1, and PTEN in the forebrain, which had previously been shown to lead to GBM with a flox/cre system $(61,62)$. This approach successfully produced GBM tumours in all mice with the triple deletion and none of the mice with any of the single deletions (60).

GEMMs, like GL261 have the major advantage of producing GBM tumours in mice without having to be immunocompromised, allowing for interactions between the tumour and the immune system to be studied. A further advantage of GEMMS is that these tumours more accurately reflect molecular changes and histology of GBMs than chemically induced tumours like GL261. The problem with GEMMs is that there are so many slightly different models of disease that it is difficult to know which is the best to use. It is likely that all GEMMs have some relevance to GBM in humans since GBM is such a heterogeneous disease. As a result, any GEMM used by researchers currently is unlikely to reflect every aspect of human GBM.

GBM is a difficult disease to model, as shown by the many different approaches used by researchers. In vitro culture of GBM cells is relatively cheap and easy, and 
does reflect some aspects of disease, but the cells are changed from the tumours they originally came from. Chemical induction of disease in animals is useful to study carcinogenesis but is not very reflective of GBM in humans. GBM is very complex and can differ substantially between patients, which makes patient derived models useful, but difficult to extrapolate from the patient they have come from. The molecular events leading up to the development of GBM are still not completely understood, which means that it is not yet known how much GEMMs accurately reflect the development of GBM in humans. 


\subsection{DNA Damage Response}

The DNA damage response (DDR) is important for the efficacy of both chemotherapy and radiation for the treatment of glioblastoma, and most other cancers. It is important to understand how this process works to understand how DNA damaging treatment of cancers work.

\subsubsection{DNA Damage Sensing}

DNA is the blueprint for the cell, which means it relies on its DNA for all the information it needs to function. DNA is under a constant barrage of attacks by environmental and intrinsic factors, like ultraviolet (UV) and ionising radiation, ROS, and spontaneous hydrolysis of nucleotides. This damage can prevent accurate replication, which can introduce mutations that lead to malignant transformation of the cells. A comprehensive DDR is required so that a cell can retain its genomic stability.

The DDR is made up of a multitude of complex processes, all of which are designed to repair specific kinds of lesions, including damaged bases, single strand breaks (SSBs) and double strand breaks (DSBs). Lesions are first detected by sensors, particularly the MRE11A/RAD50/NBS1 (MRN) complex. If the MRN complex is activated it then activates ATM-and Rad3-Related (ATR) and ataxiatelangiectasia mutated (ATM) kinases. ATR and ATM are known as master regulators of the DDR as they employ a large network of cellular processes to maintain genomic stability (63-65). ATR is activated in response to a wide variety of DNA lesions, including DSBs, and ATM is activated just in response to DSBs (66). As part of the DDR, ATR and ATM phosphorylate checkpoint 1 (CHEK1) and checkpoint 2 (CHEK2) kinases respectively, which then go on to phosphorylate P53. P53 is a key transcription factor that determines cell fate in response to DNA damage by regulating the expression of both pro- and anti-apoptotic genes.

P53 plays a key role in cancer development as mutations in P53 can inactivate it, which prevents its ability to induce cell cycle arrest or apoptosis in response to DNA damage. Cells that have mutant P53 can then go on to form a tumour, and it 
has been shown that mice with mutant forms of P53 develop an array of different tumours (67-69).

In a normal cell, once the DNA damage has been detected, there are two things that can happen next: DNA damage repair or apoptosis.

\subsubsection{DNA Damage Repair}

DNA damage repair is a complex and crucial part of the DDR, as it allows a cell to repair a range of lesions through different mechanisms and subesquently retain genomic stability of the cell, which prevents the need to apoptose.

Single base lesions like $\mathrm{N}^{7}$-methylguanine $\left(\mathrm{N}^{7}\right.$-meG) and $\mathrm{N}^{3}$-methyladenine $\left(\mathrm{N}^{3}\right.$ meA) caused by TMZ, and 8-oxoguanine (8-oxoG) and SSBs caused by ROS are removed by base excision repair (BER). BER is used to replace damaged bases and also SSBs that have been recognised by poly(ADP-ribose) polymerase family members such as PARP1 (70). BER involves the removal of the damaged base by a DNA glycosylase, followed by replacement of the base by DNA polymerase $\beta$ and sealing by the XRCC/Ligase III complex (71). BER involves lesions on only one strand of the DNA, which means that DNA polymerases always have a template to work off when replacing the damaged DNA, which reduces the error rate of this process.

DNA damage repair gets more complicated and cytotoxic once a cell sustains DSBs, which are the primary kind of DNA damage that TMZ and IR aim to inflict. There are two primary mechanisms used to repair DSBs: homologous repair (HR) and non-homologous end joining (NHEJ). NHEJ is the most common DDR pathway used by cells as it is fast and does not require a sister chromatid. However, this also means that NHEJ tends to be more error prone than HR. In the NHEJ pathway, breaks are recognised by the the Ku70/80 protein which recruits DNA-dependent protein kinase (DNA-PK), and the ends of the DSB are stabilised by these complexes. DNA-PK recruits ARTEMIS nuclease to the break and all three factors$\mathrm{Ku}, \mathrm{DNA}-\mathrm{PK}$ and ARTEMIS are phosphorylated by ATM/ATR. A number of other enzymes are recruited to the site, which includes polynucleotide kinase 3'- 
phosphatase (PKNP) and DNA polymerases $\mu$ and $\lambda$ for DNA processing. After DNA processing, the XRCC/DNA ligase IV complex seals the break $(72,73)$.

In homologous repair, heteroduplex DNA must be formed between two sister chromatids, so first the DSB is stabilised by the MRN complex, which recruits ATM to the break to start the signalling cascade. CtIP nuclease is recruited by ATM and resects the end of the DNA in combination with exonuclease I (EXO1) (7476). Replication protein A (RPA) binds to the single stranded region, which recruits ATR/ATR interacting protein (ATRIP) and then hands it off to RAD51 recombinase (RAD51), which is essential for strand invasion into the homologous sister chromatid. A heteroduplex then forms, which allows the DNA to be repaired either by crossover or non-crossover pathways (77).

\subsubsection{Apoptosis in Response to DNA Damage: The Mechanism of Treatment}

If a cell has sustained too much DNA damage to be repaired, the cell could apoptose. Apoptosis is a programmed form of cell death that usually occurs as part of normal cellular maintenance of multicellular organisms. Apoptosis, as opposed to necrosis, is a tightly controlled, non-inflammatory process.

There are two primary pathways of apoptosis: the extrinsic pathway and the intrinsic (also known as mitochondrial) pathway. Both apoptotic pathways can be induced by DNA damage. The extrinsic apoptotic pathway that is activated in response to DNA damage involves activation of the membrane receptor FasR (also known as Apo-1 or CD95) by P53 in the case of DNA damage, which leads to assembly of the death-inducing signalling complex (DISC), composed of Fasassociated protein with death domain (FADD) and pro-caspases 8 and 10. The procaspases are cleaved, which causes them to become activated, and these in turn activate executing caspases 3 and 7, known as a caspase cascade. These executing caspases are responsible for the degradation of proteins in the cell as well as activating caspase activating DNAse (CAD) which breaks down cellular DNA, leading to apoptosis $(78,79)$. 
The intrinsic, or mitochondrial apoptotic pathway is triggered by disruption of the outer membrane of mitochondria by BCL2 family proteins BCL-2-associated X protein (BAX) and BCL-2 antagonist/killer (BAK). This leads to a release of factors that trigger apoptosis, particularly cytochrome c. Cytochrome c release leads to the oligomerisation of apoptotic protease activating factor-1 (APAF-1) to form the apoptosome, which goes on to activate caspase 9 via dimerisation. Active caspase 9 then activates executing caspases 3 and 7, leading to apoptosis in the same way as the extrinsic pathway (80). It is interesting to note that there are a number of BCL2 family proteins upstream of BAX and BAK, which work in opposing ways to either activate or prevent apoptosis (81).

Different types of DNA damage lead to activation of different pathways that lead to apoptosis. IR and TMZ are used to treat GBM concurrently, and work in different ways to cause DNA damage.

Ionising radiation was the first non-surgical treatment used for GBM, and is used on the basis that rapidly proliferating tumour cells will be more susceptible to large amounts of DNA damage $(82,83)$. IR causes DNA damage by either direct attack on the DNA or by the formation of ROS within the cell, which then go on to attack the DNA and cause base modifications, as well as single and double stranded breaks (78). IR causes a wide variety of DNA lesions, so it is likely a combination of these leads to apoptosis in the cell. Double stranded breaks are accepted to be the most lethal lesion caused by IR. IR has been shown to lead to apoptosis via P53, which up-regulates the expression of Noxa. Noxa is a pro-apoptotic protein of the BCL2 family that is upstream of $\mathrm{BAX} / \mathrm{BAK}$, and activation of Noxa leads to apoptosis via the intrinsic pathway (84).

Temozolomide is an effective treatment for GBM alongside radiation and has been used as a standard of care since the publication of the Stupp et al. clinical trial in $2009(20,85)$. TMZ is a DNA alkylating agent that works by adding $\mathrm{N}^{7}-\mathrm{meG}, \mathrm{N}^{3}$ meA and $\mathrm{O}^{6}$-methylguanine $\left(\mathrm{O}^{6}-\mathrm{meG}\right)$ lesions to DNA. $\mathrm{N}^{7}$-meG and $\mathrm{N}^{3}$-meA lesions are primarily removed by BER. The $\mathrm{O}^{6}-\mathrm{meG}$ lesion is the most lethal caused by TMZ, as this leads to a mismatch to thymine instead of cytosine, which induces a 
mismatch repair loop and ultimately causes single (SSB) and double (DSB) stranded breaks (86). This lesion causes G2/M cell cycle arrest and ultimately apoptosis, via the activation of the extrinsic FasR pathway by P53 (87). $\mathrm{O}^{6}$-meG lesions can be directly repaired by the enzyme MGMT, which is quite different to the other DDR pathways as it is a single reaction to fix the lesion (88). As discussed earlier, GBM patients who have methylation of the MGMT promoter have a better response to TMZ than those without methylation (89).

Temozolomide is the chemotherapeutic drug used to treat patients with GBM, but is not widely used in in vitro experiments, as it is difficult to work with. TMZ is highly insoluble, which makes it difficult to achieve optimal concentration in vitro. As a result, experiments completed in this thesis were conducted with doxorubicin as a chemotherapeutic as it is soluble in media and subsequently much easier to work with.

Doxorubicin is used to treat many different cancers, including breast cancer, ovarian cancer and multiple myeloma (90). It is not currently used to treat GBM as it does not pass through the blood brain barrier, but much effort has been made to overcome this hurdle, and we may see successful development of a formulation of doxorubicin for GBM in the future (91-94). Doxorubicin causes DNA damage through multiple mechanisms, including topoisomerase II inhibition, DNA intercalation and oxidative damage, and is best known as a topoisomerase II inhibitor (95). Topoisomerase II performs an important function in cells as it binds DNA supercoils and entangled DNA, breaks one of the strands to unwind and release the tension on the DNA, and then reseals the two strands together. This allows cells to be able replicate and transcribe DNA effectively. Doxorubicin stops topoisomerase II from resealing the break formed after the DNA has been unwound, which leads to DSBs (96). Doxorubicin does not simply cause DNA damage; it also has a wide range of effects on the cell, which means that there are multiple pathways through which it can cause apoptosis of the cell. Doxorubicin treatment has been shown to cause increased levels of BAX in the cell, which pushes the cell towards apoptosis via the intrinsic pathway, and it can also lead to the activation of AMP-activated protein kinase (AMPK), which activates P53 leading to apoptosis via the extrinsic pathway (97). 


\subsubsection{DNA Damage and Therapy Resistance in Glioblastoma}

A key aspect of tumour formation is the series of mutations that lead to uncontrolled cell division (98). This means that DNA damage repair mechanisms were not working effectively in these cells and were subsequently unable to repair the DNA lesions that lead to these mutations. Paradoxically, in order to resist DNA damaging treatment, cancer cells, which often have a high mutational load, must be able to effectively repair or ignore this DNA damage in order to keep dividing. GBM is well known to be highly resistant to treatment, which is demonstrated by the short median survival time ( $\sim 15$ months) and time to relapse ( $\sim 6$ months) seen in patients (20). Chemo-radiation used to treat GBM damages DNA and has been shown to induce apoptosis in cells in a multitude of different ways as previously discussed, yet it seems that there are GBM cells that are able to overcome this damage and avoid apoptosis. This would indicate that GBM cells have employed mechanisms to resist DNA damage induced cell death, and understanding these mechanisms could lead to the development of more effective treatments for GBM.

MGMT expression is one of the most well documented resistance mechanisms for TMZ. GBM patients with MGMT promoter methylation have a survival benefit compared to those who do not, but most will still go on to develop treatment resistant tumours (89). There are other mechanisms that GBM tumour cells employ to resist DNA damage caused by TMZ, which include mutS homolog 6 (MSH6) mutation and alkylpurine-DNA-N-glycosylase (APNG) expression. MSH6 is a DNA mismatch repair protein, and the inactivated mutated form is selected for by TMZ treatment in GBM, which allows cells to tolerate $\mathrm{O}^{6}$-meG lesions $(99,100)$. MSH6 inactivating mutations occur in $26 \%$ of GBMs and this has been shown to be a mechanism of TMZ resistance independent of MGMT promoter methylation $(101,102)$. APNG is a base excision repair protein, that functions in the repair of $\mathrm{N}^{3}$-meA and $\mathrm{N}^{7}$-meG lesions caused by TMZ treatment. Expression of APNG in GBM cells leads to TMZ resistance and is associated with poorer survival in GBM patients with MGMT promoter methylation $(103,104)$. 
P53 is a key part of the DDR as it determines whether a cell will apoptose or repair in response to DNA damage, and when cells gain P53 mutations this leads to the formation of tumours (67-69). Brennan et al. showed that the P53 pathway was dysregulated in $85.3 \%$ of tumours through mutations/deletion of TP53, amplification of $M D M 1 / 2 / 3$, or deletion of cyclin dependent kinase inhibitor $2 \mathrm{~A}$ $(C D K N 2 A)(28)$. Given this information, it is likely that P53 plays a significant role in resistance to DNA damaging treatment of GBM, as mutant P53 allows cells to tolerate large amounts of DNA damage and continue proliferating.

The EGFR is a receptor tyrosine kinase that is frequently altered in GBM (57\% of all GBM, $\sim 80 \%$ of classical subtype). The most common mutation is the EGFRvIII activating mutation, which leads to constitutive activation of the survival pathways downstream of EGFR (28). The PI3K/AKT/MTOR (mammalian target of rapamycin) and RAS/MEK/ERK (extracellular signal-regulated kinase) pathways are both activated via EGFR and among many other downstream effects, activation of both pathways lead to an increase in DNA repair activity. This increase in DNA repair activity results in increased cell survival and proliferation $(105,106)$. As shown by Brennan et al., $89.6 \%$ of all GBM tumours analysed had at least one alteration in the PI3K pathway, which highlights that this is a key pathway for therapy resistance in GBM (28). In addition to constitutive activation of EGFR in GBM, the EGFR can be activated by IR, which leads to activation of downstream signalling pathways, leading to radioresistance $(107,108)$. IR can also lead to EGFR translocation to the nucleus where it activates DNA-PK, which in turn activates DSB repair, leading to cell survival and therapy resistance $(109,110)$. As a result of this evidence, several small-molecule EGFR inhibitors have been trialled in GBM, including erlotinib and gefitinib, with no great success so far (111). Trials of new inhibitors are ongoing.

GBM has many other characteristics that contribute to therapy resistance, which do not directly involve the DDR. These include glioma stem cells and the tumour microenvironment.

GBMs are well-documented to have a stem cell population that contributes to therapy resistance $(112,113)$. These cells have many of the characteristics of noncancerous stem cells: self renewal, expression of stem cell markers and a low rate of 
proliferation, and their function is to maintain the tumour cell population $(114,115)$. Glioma stem cells that have been selected for have been shown to accurately recapitulate GBM tumours in mice, which reinforces their role as cells that maintain the tumour cell population (41-43). As a result, GSCs have been studied extensively to find ways of targeting these cells with treatment (115-117).

The tumour microenvironment plays a large role in therapy resistance in GBM. Tumour vasculature is highly disorganised, leading to hypoxia and necrosis in various areas of the tumour (118). The problem with this is twofold: hypoxia is radioprotective, as oxygen is required to create ROS to cause DNA damage, and any drugs against the tumour that are able to pass through the blood brain barrier (BBB) are unable to reach the majority of the tumour as there is no vasculature to take them there (119). Hypoxia is not the only problem with the microenvironment, as tumour infiltrating macrophages are recruited to the tumour and create an extremely immunosuppressive environment (120). This makes immunotherapeutic approaches to treatment difficult. Given the success of checkpoint inhibitor immunotherapies anti-CTLA4 (ipilimumab) and anti-PD1 (nivolumab/pembrolizumab) in other cancers, this is still a large and developing area of GBM research (121-123).

Many factors contribute to therapy resistance in any GBM tumour, and these are all inter-related, making it impossible to tie therapy resistance to just one protein, signalling pathway or cell type. When paired with the large amount of heterogeneity both within and between GBM tumours, this leads to a large number of possible resistance mechanisms at a tumours disposal. These resistance mechanisms can also interact in many complex ways, making therapy resistance itself difficult to target.

\subsubsection{Other Therapeutic Approaches}

There has been little change to the treatment regimen and prognosis for GBM patients in the past $30+$ years (111). As a result, a large part of research into GBM is in the development of new treatments. 
Bevacizumab is an anti-vascular endothelial growth factor (VEGF) monoclonal antibody that has been extensively studied, and is an option available for the treatment of GBM. GBM has high expression of VEGF-A, which plays a large part in the abnormal vascularity of GBM tumours (124). Bevacizumab is a humanised monoclonal antibody against VEGF-A that prevents VEGF-A from interacting with its receptor, and this leads to vascular normalization in the tumour, which allows for more effective chemotherapy and radiation (125). Following a number of successful phase I and II clinical trials, the US Food and Drug Administration (FDA) approved bevacizumab for the treatment of GBM in 2009 via a fast-tracked process, but it is not yet approved for use in Europe (126-128). Two phase III clinical trials were completed and reported in 2014 for the treatment of GBM with bevacizumab: the American Radiation Therapy Oncology Group (RTOG) 0825 trial, and the European Avastin in Glioblastoma (AVAGlio) trial $(129,130)$. Both trials showed similar results, with improved progression free survival with bevacizumab alongside standard treatment, but no major improvement in overall survival. Bevacizumab is currently used in the clinic to treat GBM, but given the results of the phase III clinical trials this is not routine (131).

Treating GBM with immune-based therapies is an increasingly popular area of research, fuelled by the recent success of immunomodulatory agents anti-CTLA4 and anti-PD1 for treatment of metastatic melanoma- a disease that is highly resistant to conventional treatment (132). There are multiple vaccination approaches being tested that are trying to activate the immune system against the tumour, which include adoptive transfer of autologous dendritic cells (DCs) or chimeric antigen receptor (CAR) T-cells, synthetic tumour antigen vaccines, and immunomodulators like anti-PD1 $(122,133)$. Each of these approaches hold promise for the future treatment of GBM, but so far have not made it past early phase clinical trials.

One of the most revolutionary new treatments for GBM is called "Tumor Treating Fields" (TTF). Recently, R. Stupp has published results of a phase III clinical trial, which shows clinical benefit for the use of TTF (134). TTF is a unique modality of treatment, which involves the placement of a cap over the scalp that contains a series of electrodes that run alternating electric fields through the brain of the patient at high frequency (135). These electric fields are undetectable to the person 
being treated. TTF disrupt the mitotic spindle in dividing cells, particularly affecting anaphase and cytokinesis, leading to aberrant mitotic exit (136). TTF presents a unique mechanism of action compared to other therapeutic approaches, and proposed mechanisms of resistance, primarily cell size, could be easily overcome by adjustment of the frequency of the device (137). TTF has been approved for use by the FDA and it is likely that TTF will become standard of care alongside adjuvant TMZ for treatment of GBM in the near future $(138,139)$.

Development of treatments for GBM is ongoing, with many clinical trials currently registered with a variety of approaches (111). One of the main problems with clinical trials for GBM is the relatively low frequency of GBM in the population, meaning recruitment for trials takes a long time, and with most trials failing this means that time and money are being wasted on drugs that don't work. As a result of this, a new trial system has been developed for GBM called the Adaptive Global Innovative Learning Environment for Glioblastoma (GBM AGILE) (140). This is an adaptive phase II/III clinical trial, that allows the testing of multiple treatments at one time and consists of two stages. The first stage pairs multiple treatments with a common control. If a treatment does well, it moves to the second stage, where it will be tested on a larger cohort of patients. If a treatment does not do well, it will be dropped from the trial. The idea is that this unique trial design allows for rapid identification of beneficial therapeutics for GBM and helps get them to phase III trials and FDA approval quicker. 


\section{$1.3 \quad$ B-cell Lymphoma 6}

GBM is a disease that is highly resistant to the DNA-damaging therapies used to treat it, namely ionising radiation and temozolomide. Many avenues of therapy resistance have been investigated for GBM, and a new potential contributor to GBM therapy resistance is B-cell lymphoma 6 (BCL6).

\subsubsection{Discovery of BCL6 in B-cell Lymphoma}

Diffuse large B-cell lymphoma (DLBCL) is the most common form of lymphoma, and BCL6 was first discovered in DLBCL due to the translocation of the BCL6 gene on chromosome 3q27 (141-144). BCL6 is a $95 \mathrm{kDa}$ protein and a member of the bric-a-brac, tramtrack, broad complex/pox virus zinc finger (BTB/POZ) family of transcription factors. BCL6 is generally seen to be an oncogenic protein, as it represses genes involved in apoptosis and the DNA damage response, and thus prevents these processes from happening, for example TP53 (145), ATR (146) and CHEK1 (147). Different types of B-cell lymphomas have high levels of BCL6 expression, and are hugely dependent on BCL6 expression for maintenance of tumour cells, particularly DLBCL and follicular lymphoma (FL) (148). Conversely, BCL6 also represses pro-oncogenic genes like BCL2 (149) and c-MYC (150), which is thought to be a mechanism to balance out the pro-oncogenic properties of BCL6 and prevents transformation of B-cells into B-cell lymphoma (151). When lymphoma develops, it is often due to promoter mutations and chromosomal translocations of pro-oncogenic genes like BCL2 and $c-M Y C$ which circumvents their inhibition by BCL6 (152). This then combines with the pro-oncogenic actions of BCL6 and leads to the transformation of B-cells into lymphoma.

Since the discovery of the key role of BCL6 in lymphoma, other types of haematological tumours have been looked at for BCL6 expression and involvement in tumour development. Acute lymphoblastic leukaemia (ALL) has been shown to have BCL6 involvement in tumour development and resistance to treatment $(153,154)$. 


\subsubsection{BCL6 Structure and Function}

BCL6 is characterised by a N-terminus BTB/POZ domain, a central PEST domain, and 6 C-terminal zinc finger DNA-binding motifs (Figure 1.1) (155). BCL6 must dimerise to be functional and the $\mathrm{BTB} / \mathrm{POZ}$ domain is required for this dimerisation to occur. The BTB/POZ domain is the primary location for BCL6 to recruit co-repressors, which is how it carries out its transcriptional repressor activity.

The most well-known mechanism for transcriptional repression with BCL6 is the recruitment of BCL6 co-repressor (BCOR) (156,157), nuclear co-repressor 1 (NCOR) (158), or silencing mediator for retinoid or thyroid-hormone receptors (SMRT, also known as NCOR2) (159).

BCOR was first characterised by Huynh et al. as a BCL6 specific co-repressor (156). BCOR forms a polycomb repressor complex 1 (PRC1)-like complex with polycomb group ring finger 1 (PCGF1), lysine demethylase 2B (KDM2B), ring finger protein 1 (RING1), S-phase kinase associated protein 1 (SKP1), RING1 and YY1 binding protein (RYBP) and ring finger protein 2 (RNF2) (160-163), and binds a completely different part of the BTB/POZ domain to NCOR and SMRT $(157,164)$. NCOR and SMRT form a complex with transducin $\beta$-like 1 (TBL1), TBL1-related protein (TBLR1), G protein pathway suppressor 2 (GPS2) and histone deacetylase 3 (HDAC3), and enhance HDAC3 deacetylation activity (165). BCOR and NCOR/SMRT can be simultaneously recruited by BCL6 to repress transcription by histone deacetylation at the transcription start site. BCL6 can also recruit SMRT alone to enhancers, which leads to $\mathrm{H} 3 \mathrm{~K} 27$ de-acetylation via HDAC3, and also shuts down gene expression downstream. Both of these events can happen simultaneously in one cell at different loci and this shows that BCL6 is capable of different mechanisms of gene repression (164).

The PEST domain (also known as regulatory domain 2 (RD2) or the "middle domain") can recruit co-repressors metastasis associated 1 family member 3 (MTA3) and c-terminal binding protein 1 (CTBP1). When MTA3 is recruited to the PEST domain it leads to the repression of PR/SET domain 1 (PRDM1), a gene involved in plasma cell differentiation $(166,167)$. CTBP1 is involved in 
autoregulation of BCL6 (168). The PEST domain is also a key site for inactivation of the BCL6 protein via both acetylation by E1A binding protein P300 (EP300) and phosphorylation by mitogen activated protein-kinase 1 (MAPK), which leads to BCL6 degradation $(169,170)$.

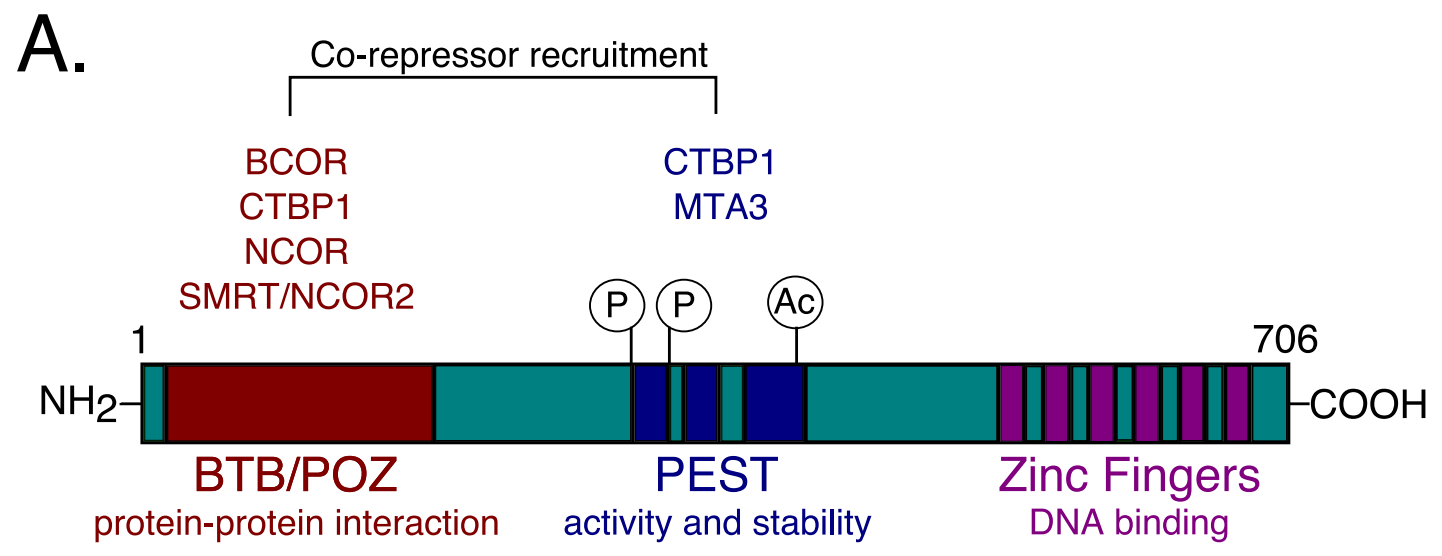

B.

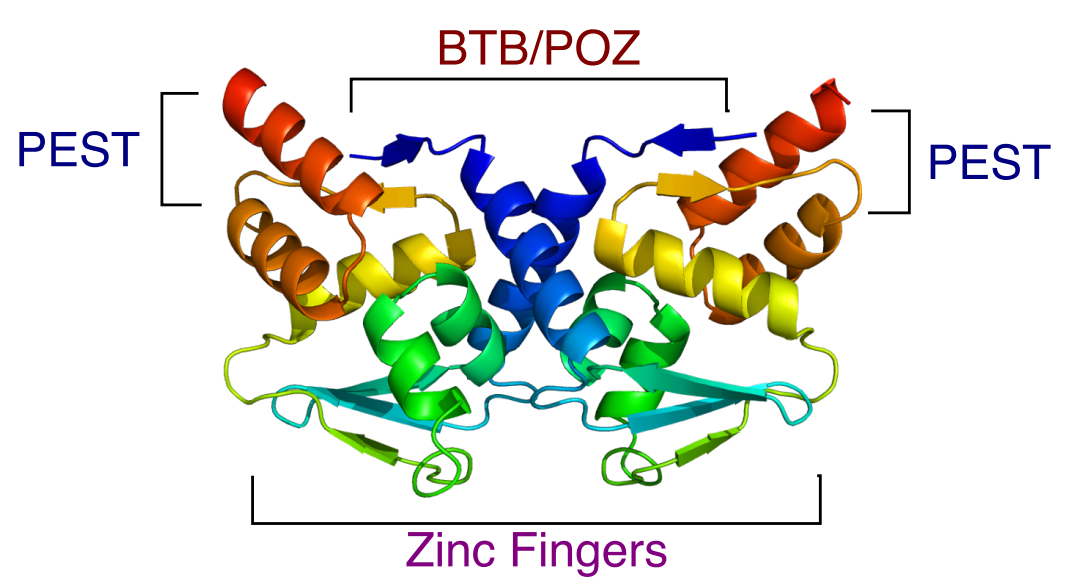

Figure 1.1: Structure of BCL6. (A) Schematic representation of BCL6 functional domains, showing where co-repressors are recruited and the phosphorylation/acetylation domains. Adapted from Basso and Dalla-Favera (155). (B) 3D representation of BCL6 homodimer, showing the functional domains. 3D image adapted from File:Protein BCL6 PDB 1r28.png/User:Enw/Wikimedia Commons/CC-BY-SA 3.0.

Shortly after the discovery that BCL6 plays a key role in the development of B-cell lymphoma, it was shown that BCL6 is crucial for the formation of germinal centers (GC) (171). GCs are formed in lymphoid organs like lymph nodes or the spleen, and are where B-cells mature to create more specific antibodies against the agent of infection, or antigen. The formation of GCs is a key part of the immune response to infection. The reason why BCL6 plays a key role in GCs is that B-cell maturation in 
the germinal center requires two key processes: somatic hypermutation (SHM) and class switch recombination (CSR). SHM is the process of B-cells further mutating the variable region of the antibodies they produce, in an effort to produce more specific antibodies than they produced before. CSR is the process of changing the class of antibody B-cells produce, to one more specific for the type of antigen that has been encountered. Both SHM and CSR require activation-induced cytidine deaminase (AID) to induce DNA breaks, and BCL6 allows B-cells to tolerate this genomic instability by repressing expression of differentiation (BLIMP1, IRF4, PRDM1), pro-apoptotic (BCL2, NFKB1), and DNA damage response genes (ATR, TP53) $(148,155,167,172,173)$. As a result, BCL6 expression is crucial for the germinal center reaction, as it prevents apoptosis in response to DNA damage and it also prevents differentiation and activation of B-cells before they leave the germinal center, which allows B-cells to develop high affinity antibodies against the infection that the body is fighting.

BCL6 has also been shown to play an essential role in the differentiation of Tfollicular helper (Tfh) cells (174-176). Tfh cells are important for the formation of germinal centers, and the maturation of GC B-cells into memory B-cells or plasma cells. Tfh cells interact with GC B-cells after they have gone through SHM and ensure that the B-cells that have the highest affinity B-cell receptors (BCR) are retained in the GC (177). This process is essential to ensure the B-cells that mature from the GC produce the most effective antibodies.

As a result of BCL6's crucial role in the development of lymphoma, much investigation has gone into therapies that could inhibit the action of BCL6, with the primary focus of research being on preventing the interaction between BCL6 and its co-repressors. It was discovered that mice that had a mutation in the BCL6 $\mathrm{BTB} / \mathrm{POZ}$ domain that prevents co-repressor recruitment, were unable to form germinal centers (GCs), but were otherwise healthy. This was in complete opposition to BCL6 knockout mice, which suffered from lethal inflammatory disease (178). This finding encouraged the approach of developing inhibitors that bound in the BTB/POZ domain and prevented co-repressor recruitment. One of the most recently developed inhibitors with this mechanism of action is called FX1. FX1 has been shown to have a stronger affinity for the BTB/POZ binding domain 
than the BCL6 co-repressors and induced regression of DLBCL xenografts in mice (179). Investigations are ongoing with FX1 and there is a hope it will go to clinical trial in the future.

\subsubsection{Role of BCL6 in Solid Tumours}

Solid tumours have been investigated for BCL6 involvement with their development, and the most established tumour type in this area is breast cancer. BCL6 is highly expressed in breast tumours, as over 50\% have BCL6 amplification (180), and BCL6 expression has been shown to increase proliferation, migration and invasion of breast cancer cell lines (181). BCL6 is also correlated with disease progression and survival, and promotes epithelial-to-mesenchymal transition (EMT) in breast cancer by activating transcription of zinc finger E-box binding homeobox 1 (ZEB1), an EMT marker (182).

Many other solid tumours have been investigated for BCL6 involvement, and much of this research is in its infancy. Recently, it has been shown that BCL6 expression correlates closely with downregulation of TGF- $\beta$ signalling in non-small cell lung cancer (NSCLC), and combination inhibition of STAT3 and BCL6 significantly reduced tumour growth in a subset of these tumours (183). Increased BCL6 protein has been shown to predict poor prognosis in ovarian cancer (184), and has also been associated with an increased grade of colorectal cancer (185). These reports of BCL6 in solid tumours are few and far between, with almost no follow up papers published on any of the studies discussed here. All of these studies have limitations to their approach, so it is difficult to know if BCL6 is playing a significant role in these tumours until there is further evidence reported on the role of BCL6 in these tumours.

\subsubsection{BCL6 and GBM}

Given the fundamental role that BCL6 plays in the germinal center to help cells resist death in response to DNA damage, its involvement in the development of DLBCL, and newly described involvement in solid tumours such as breast cancer, is 
it possible that BCL6 expression is being utilised by GBM cells to resist DNA damage?

In 2014, Ruggieri et al. reported that BCL6 translocations had an association with IDH1 mutant GBM (186). Sections of patient tumours taken prior to treatment were stained for IDH1 expression and fluorescence in-situ hybridisation (FISH) analysis for BCL6 translocation. Immunohistochemistry (IHC) and quantitative polymerase chain reaction (qPCR) was also performed to assess BCL6 expression and P53 status. All tumours that had BCL6 translocations were positive for IDH1 expression, had BCL6 expression, and mutant/wild type P53 (11/30 GBM tumours). The authors compared their glioblastoma samples to less aggressive grade II and III astrocytoma samples and found that the lower grade gliomas had lower frequency of BCL6 translocation and expression (5/36 tumours, versus 11/30). Subsequently, the authors argued that BCL6 translocation and expression correlated with the aggressiveness of the tumour. IDH1 expression is generally associated with a secondary form of GBM, which makes up a small proportion of total GBMs, but it is unclear from this publication if the tumours that came up positive for both IDH1 mutation and BCL6 translocation were defined as secondary GBMs. The findings in this paper suggest that BCL6 may be important in secondary, IDH1 positive GBMs.

In 2017, Xu et al. published a significant report into the mechanism of action and in vivo targetability of BCL6 in GBM (187). They showed that BCL6 is upregulated in both established GBM cell lines (DBTRG, T98G, U87-MG, U138, U251, U343) as well as their own primary tumour cell lines, and expressed at similar levels to breast cancer cell lines. BCL6 knockdown with short hairpin RNA (sh-RNA) in GBM cell line U87-MG lead to decreased growth of subcutaneous tumours, and inhibition of BCL6 with retroinverso BCL6 peptide inhibitor (RI-BPI) also decreased growth of a primary cell line set of tumours grown subcutaneously. To look at the role of BCL6 in vivo, they used the KrasG12V genetic model of GBM, where EGFPKrasG12V plasmids are transfected into neural precursor cells via electroporation, which leads to the formation of a tumour. When this plasmid was coupled with a BCL6 sh-RNA, a significant reduction in tumour growth and increase in survival was seen in these mice, which indicated that BCL6 was playing a significant role in 
the development of these tumours. Chromatin immunoprecipitation and sequencing (ChIP seq) revealed a new potential target gene of BCL6 in GBM: AXL receptor tyrosine kinase (AXL), which is upregulated in GBM. Interestingly, they found that BCL6 binding at the AXL locus correlated with RNA polymerase II binding, which indicated that BCL6 may activate transcription of AXL, leading to upregulation of downstream pathways such as MEK/ERK and PI3K/AKT, which are commonly upregulated in GBM. The findings in this paper suggest that BCL6 plays an important role in GBM and is a potential new therapeutic target.

These two papers provide some evidence that BCL6 plays a significant role in the biology of GBM, and it may prove to be a good therapeutic target. 


\subsection{Aims of this Study}

This introduction has outlined the challenges of treating Glioblastoma, a highly heterogeneous disease with a dismal prognosis that is resistant to current therapeutic approaches. Treatment for GBM hasn't changed significantly in 30+ years, and there is a desperate need for new therapeutic approaches. There is some evidence of BCL6 playing a role in GBM, and given that GBM cells are highly resistant to DNA damage, it is possible that BCL6 plays a role in this part of therapy resistance. This thesis will evaluate whether BCL6 is playing this role in GBM.

Aim 1: Determine that BCL6 is present and transcriptionally active in GBM cells.

Aim 2: Investigate BCL6 binding to DNA and BCL6 target genes in GBM cells.

Aim 3: Investigate the effect of BCL6 inhibition on global transcription in GBM cells. 


\section{$2 \quad$ Materials and Methods}

\section{$2.1 \quad$ Reagents}

\section{Cell Culture}

$100 \mathrm{~mm}$ dishes

$100 \mathrm{mM}$ pyruvate

12 well plates

$150 \mathrm{~mm}$ dishes

6 well plates

96 well solid white flat bottom plates

Dulbecco's Phosphate-Buffered Saline (DPBS)

eMyco-mycoplasma detection PCR kit

Foetal Bovine Serum (FBS)

Roswell Park Memorial Institute-1640 media

(RPMI-1640)

T25 flasks

T75 flasks

Trypan Blue

Trypsin-EDTA $0.05 \%$
Corning, NZ

Life Technologies, NZ

Corning, NZ

Corning, NZ

Corning, NZ

Corning, NZ

Life Technologies, NZ

iNtron Biotech, Korea

Life Technologies, NZ

Life Technologies, NZ

Corning, NZ

Corning, NZ

Life Technologies, NZ

Life Technologies, NZ

\section{Bacteria}

Ampicillin

LB Broth

One Shot ${ }^{\circledR}$ OmniMAX ${ }^{\mathrm{TM}} 2$ T1R Chemically Competent

E. coli
Sigma-Aldrich, NZ

Life Technologies, NZ

Life Technologies, NZ

\section{Chemicals/Miscellaneous}

1,2-diaminocyclohexane-N,N,N',N,-tetraacetic acid

100 bp marker

$30 \%$ Acrylamide solution

37\% Formaldehyde

Acetic acid

Agarose
Sigma-Aldrich, NZ

Life Technologies, NZ

BioRad, NZ

Sigma-Aldrich, NZ

Univar, USA

Life Technologies, NZ 
Ammonium Persulphate (APS)

BioRad, NZ

Bovine Serum Albumin (BSA)

ICP Biologicals, NZ

Complete protease inhibitor

Sigma-Aldrich, NZ

Dithiothreitol

Sigma-Aldrich, NZ

Doxorubicin

Calbiochem, NZ

Ethylene glycol-bis( $\beta$-aminoethyl ether)-N,N,N',N'-tetraacetic acid (EGTA)

Ethylenediaminetetraacetic acid (EDTA)

Glycerol

Sigma-Aldrich, NZ

HEPES

Hydrochloric acid

Calbiochem, NZ

Thermo Fisher, NZ

Thermo Fisher, NZ

IGEPAL

J.T.Baker, USA

Isopropanol (2-propanol)

Sigma-Aldrich, NZ

Ny'Tran SuPer Charge Nylon Membrane

Sigma-Aldrich, NZ

Orange G

Sigma-Aldrich, NZ

PageRuler Plus Prestained Protein Ladder

Sigma-Aldrich, NZ

Parafilm

Thermo-Fisher, NZ

Phosphoric acid

Thermo Fisher, NZ

Polyvinylidene Fluoride (PVDF) membrane

Merck-Millipore, NZ

Ponceau S

BioRad, NZ

Potassium hydroxide

Sigma-Aldrich, NZ

Protein A Agarose

Thermo Fisher, NZ

Protein G Dynabeads ${ }^{\circledR}$

Sigma-Aldrich, USA

Sodium chloride

Life Technologies, NZ

Sodium deoxycholate (Na-DOC)

Thermo Fisher, NZ

Sodium dodecyl sulphate (SDS)

Sigma-Aldrich, NZ

SYBRTM Safe

Calbiochem, NZ

TEMED

Life Technologies, NZ

Tris

BioRad, NZ

Triton X-100

Sigma-Aldrich, NZ

Tween 20

Thermo Fisher, NZ

Viafect

Thermo-Fisher, NZ

Promega, USA 
Kits

$\begin{array}{ll}\text { Agilent RNA 6000 Nano kit } & \text { Agilent, NZ } \\ \text { BioRad protein assay } & \text { BioRad, NZ } \\ \text { iScript }{ }^{\mathrm{TM}} \text { cDNA Synthesis kit } & \text { BioRad, NZ } \\ \text { KAPA SYBR }{ }^{\circledR} \text { FAST Universal One-Step qRT-PCR kit } & \text { KAPA Biosystems, USA } \\ \text { LightShift Chemiluminescent EMSA kit } & \text { Life Technologies, NZ } \\ \text { LTX and PLUS } & \text { Thermo-Fisher, NZ } \\ \text { Luciferase Assay System 10 pack } & \text { Promega, USA } \\ \text { MinElute PCR purification kit } & \text { Qiagen, USA } \\ \text { PCR purification kit } & \text { Qiagen, USA } \\ \text { Phusion High-Fidelity PCR kit } & \text { Thermo-Fisher, NZ } \\ \text { Purelink HiPure Plasmid Maxiprep kit } & \text { Life Technologies, NZ } \\ \text { Qubit quantification assays } & \text { Life Technologies, NZ } \\ \text { Restore Western Blot Stripping buffer } & \text { Pierce, NZ } \\ \text { Western Lightning Ultra } & \text { Perkin Elmer, USA } \\ \text { Zymo Quick RNA MiniPrep kit } & \text { Zymo Research, USA }\end{array}$

\subsection{Cell Culture}

\subsubsection{Cell Lines}

Human glioblastoma cell lines LN18 and U87-MG were obtained from the American Type Culture Collection (USA). The human leukaemia cell line K562 was a gift from Michael Berridge, Malaghan Institute of Medical Research. The human lymphoma cell line Raji was a gift from Ian Morison, University of Otago.

\subsubsection{Cell Culture Media}

LN18 and U87-MG for the luciferase assay and K562 cells were grown in RPMI 1640 media supplemented with 5\% (v/v) heat inactivated FBS. LN18 and U87-MG for all other assays were grown in RPMI-1640 supplemented with 10\% (v/v) FBS (complete media). Raji cells were grown in RPMI-1640 media supplemented with $5 \%(\mathrm{v} / \mathrm{v})$ FBS and $1 \mathrm{mM}$ pyruvate. 


\subsubsection{Cell Culture}

All cell lines were grown as adherent cultures and were grown to 70-90\% confluency before passage. All cells were grown in a SANYO MCO-20AIC $\mathrm{CO}_{2}$ Incubator, the environment was humidified and maintained at $37^{\circ} \mathrm{C}$ with $5 \% \mathrm{CO}_{2}$. All cultures were passaged under sterile conditions in a Labconco Purifier Biological Safety Cabinet. To passage cultures, media was aspirated and cells were washed with DPBS, followed by addition and aspiration of trypsin-EDTA $0.05 \%$ and incubated at $37^{\circ} \mathrm{C}$ for 5 minutes. Trypsin was inactivated and cells were put into suspension by washing the surface with RPMI complete media. Cell counting was performed using Trypan Blue to determine cell viability and a haemocytometer. Checks were performed every 2-3 months to ensure cells were mycoplasma free using eMycomycoplasma detection PCR kit.

\subsection{Plasmid Preparation}

\subsubsection{Bacterial Transformation and Culture}

A tube of One Shot ${ }^{\circledR}$ OmniMAX ${ }^{\mathrm{TM}} 2$ T1R chemically competent Escherichia coli were thawed on ice and $5 \mu \mathrm{l}$ of plasmid was added to the cells. The mixture was incubated on ice for 20 minutes, heated to $42{ }^{\circ} \mathrm{C}$ for 90 seconds, then put on ice for another 2 minutes. $250 \mu \mathrm{l}$ of LB broth was added to the mixture, and the mixture was transferred to a $50 \mathrm{ml}$ tube. The mixture was then placed in a shaking incubator at $37^{\circ} \mathrm{C}$ for approximately 45 minutes, or until the culture had sufficiently grown. Once the culture had expanded, $50 \mu \mathrm{l}$ of the culture was spread onto a prepared Luria broth-ampicillin (LB-Amp) agar plate $(50 \mathrm{mg} / \mathrm{ml})$ and incubated at $37{ }^{\circ} \mathrm{C}$ overnight. After overnight incubation, one single colony was selected and put in 5 $\mathrm{ml}$ of LB-Amp $(50 \mathrm{mg} / \mathrm{ml})$ media and placed in a shaking incubator at $37^{\circ} \mathrm{C}$ until bacterial growth reached the exponential phase. Once the bacteria had sufficiently grown, the culture was transferred into $500 \mathrm{ml}$ of LB-Amp media in a large conical flask and placed in a shaking incubator at $37^{\circ} \mathrm{C}$ overnight, until the culture had sufficiently grown. Once the culture had sufficiently grown, the bacteria were pelleted by centrifugation and prepared for plasmid extraction. 


\subsubsection{Plasmid Preparation and DNA Precipitation}

Preparation of the plasmids was completed using the PureLink ${ }^{\mathrm{TM}}$ HiPure Plasmid Maxiprep Kit, according to the manufacturers instructions. Briefly, the HiPure Maxi column was equilibrated, while the bacteria were pelleted by centrifugation (4000 xg for 10 minutes). The cells were resuspended until homogenous in $10 \mathrm{ml}$ Resuspension Buffer with RNase A and $10 \mathrm{ml}$ of Lysis Buffer was then added. The tube was mixed gently by inversion until homogenous and incubated at room temperature for 5 minutes. To precipitate the lysate, $10 \mathrm{ml}$ of precipitation buffer was added and mixed until homogenous. The lysate was then clarified by transfer of the lysate to the column, and allowed to filter through by gravity flow. The flow through was passed through the column once more before the inner filtration cartridge of the column was discarded. To wash the column, $50 \mathrm{ml}$ of Wash Buffer was added and allowed to drain by gravity flow, the flow through was discarded. The DNA was eluted from the column by the addition of $15 \mathrm{ml}$ Elution Buffer. The DNA was precipitated with the PureLink ${ }^{\text {TM}}$ HiPure Precipitator Module, according to the manufacturers instructions. Briefly, $10.5 \mathrm{ml}$ isopropanol was added to the eluate and incubated for 2 minutes at room temperature (RT). The plunger was revoved from a $30 \mathrm{ml}$ syringe and the PureLink ${ }^{\text {TMHiPure Precipitator was }}$ attached to the end of the syringe. The precipitated DNA mixture was added to the syringe and the plunger was then used to slowly pass the eluate through the precipitator. The flow through was discarded and the precipitator detatched from the end of the syringe. The plunger was removed again from the syringe and the preciptator re-attached to the end of the syringe. To wash the precipitator, $5 \mathrm{ml}$ of $70 \%$ ethanol was added to the syringe and pushed through the precipitator using the plunger. To remove the ethanol from the precipitator, the precipitator was taken off the end of the syringe, the plunger removed, the precipitator re-attached and air was then blown through the precipitator by pushing the plunger in the syringe. To elute the DNA, the precipitator was attached to the end of a $5 \mathrm{ml}$ syringe which had already had the plunger removed. The precipitator was placed over a sterile $1.5 \mathrm{ml}$ centrifuge tube and $1 \mathrm{ml}$ of TE buffer was added to the syringe and pushed through the precipitator with the plunger. The DNA was then quantified with the Broad Range reagents as detailed in 2.4.1. 


\subsection{DNA, RNA and Protein Quantification}

\subsubsection{DNA Quantification}

The Qubit ${ }^{\mathrm{TM}}$ Fluorometer was used for DNA quantification. The DNA Broad Range Assay was used for plasmids and the High Sensitivity Assay was used for all other samples. These assays were used according to the manufacturers instructions. Briefly, the two DNA standards were brought to room temperature while the working solution was prepared. Working solution was prepared as a 1:200 dilution of the Broad Range/ High Sensitivity DNA Qubit ${ }^{\mathrm{TM}}$ Reagent in Broad Range/ High Sensitivity DNA Qubit ${ }^{\mathrm{TM}}$ Buffer. $200 \mu \mathrm{l}$ of working solution was prepared per sample, including the standards. For the standards, $190 \mu \mathrm{l}$ of working solution was added to a Qubit ${ }^{\mathrm{TM}}$ Assay Tube followed by $10 \mu \mathrm{l}$ of the standard. For the sample, $199 \mu \mathrm{l}$ of working solution was added to a Qubit ${ }^{\mathrm{TM}}$ Assay Tube, followed by $1 \mu \mathrm{l}$ sample. Once standard or sample was added to the working solution, tubes were vortexed briefly and incubated for 2 minutes at RT. Once incubated, the standards were first read by the Qubit ${ }^{\mathrm{TM}} 2.0$ Fluorometer to create a standard curve. Once the standard curve was created, samples were read by the fluorometer and the dilution calculator was used to determine the stock concentration.

\subsubsection{RNA Quantification}

The Qubit ${ }^{\mathrm{TM}}$ Fluorometer and RNA High Sensitivity Assay was used for RNA quantification. The RNA High Sensitivity Assay was used according to the manufacturers instructions. The two RNA standards were brought to room temperature while the working solution was prepared. Working solution was prepared as a 1:200 dilution of the High Sensitivity RNA Qubit ${ }^{\mathrm{TM}}$ Reagent in High Sensitivity RNA Qubit ${ }^{\mathrm{TM}}$ Buffer. $200 \mu \mathrm{l}$ of working solution was prepared per sample, including the standards. For the standards, $190 \mu \mathrm{l}$ of working solution was added to a Qubit ${ }^{\mathrm{TM}}$ Assay Tube followed by $10 \mu \mathrm{l}$ of the standard. For the sample, $199 \mu \mathrm{l}$ of working solution was added to a Qubit ${ }^{\mathrm{TM}}$ Assay Tube, followed by $1 \mu \mathrm{l}$ sample. Once standard/sample was added to working solution, tubes were vortexed briefly and incubated for 2 minutes at RT. Once incubated, the standards were first read by the Qubit ${ }^{\mathrm{TM}}$ 2.0 Fluorometer to create a standard curve. Once the standard curve was created, samples were read by the fluorometer and the dilution calculator 
was used to determine the stock concentration. As the RNA High Sensitivity Assay has a limited range, sometimes a dilution of the original sample was used.

\subsubsection{Protein Quantification}

The Qubit ${ }^{\mathrm{TM}}$ Fluorometer and Protein Assay was used for protein quantification. The Protein assay was used according to the manufacturers instructions. The two RNA standards were brought to RT while the working solution was prepared. Working solution was prepared as a 1:200 dilution of the Protein Assay Qubit ${ }^{\mathrm{TM}}$ Reagent in Protein Assay Qubit ${ }^{\mathrm{TM}}$ Buffer. $200 \mu$ l of working solution was prepared per sample, including the standards. For the standards, $190 \mu \mathrm{l}$ of working solution was added to a Qubit ${ }^{\mathrm{TM}}$ Assay Tube followed by $10 \mu \mathrm{l}$ of the standard. For the sample, $199 \mu \mathrm{l}$ of working solution was added to a Qubit ${ }^{\mathrm{TM}}$ Assay Tube, followed by $1 \mu \mathrm{l}$ sample. Once standard/sample was added to working solution, tubes were vortexed briefly and incubated for 2 minutes at RT. Once incubated, the standards were first read by the Qubit ${ }^{\mathrm{TM}}$ 2.0 Fluorometer to create a standard curve. Once the standard curve was created, samples were read by the fluorometer and the dilution calculator was used to determine the stock concentration.

\subsection{Transient Transfection}

\subsubsection{Plasmids}

\begin{tabular}{ll}
\hline BCL64-tkLUC(1) & Prof. Ari Melnick, Weill Cornell Medical College, NY, USA \\
BCL6wt & Prof. Ari Melnick, Weill Cornell Medical College, NY, USA \\
pcDNA3-GFP & Prof. Ari Melnick, Weill Cornell Medical College, NY, USA \\
PGL3 & Promega, USA
\end{tabular}

\subsubsection{LTX and Plus Transfection}

For initial transfections in the development of the luciferase assay, LTX and plus reagent was used, and the transfection media was prepared according to manufacturers instructions. Briefly, $7.5 \times 10^{4}$ cells were seeded into a 12 well plate 24 hours before transfection. For transfection, $2.5 \mu \mathrm{g}$ of plasmid DNA was mixed 
with $2.5 \mu \mathrm{l}$ PLUS reagent in $500 \mu \mathrm{l}$ RPMI without serum per well. This was left to complex for 5 minutes, before $2.5 \mu \mathrm{LTX}$ reagent was added to the mixture and allowed to complex for 20 minutes. The complex was then added to the cells in a final serum concentration of $1 \%(\mathrm{v} / \mathrm{v})$ for 4 hours. After 4 hours the complex was removed and RPMI media with 5\% (v/v) serum was added to the wells.

\subsubsection{Viafect Transfection}

For all transfections, Viafect reagent was used according to the manufacturers instructions. Briefly, cells were plated to achieve $70 \%$ confluence 24 hours after plating. Cells were transfected 24 hours after plating. For transfection, $2.5 \mu \mathrm{g}$ of plasmid was mixed with $5 \mu$ l of Viafect reagent in $500 \mu$ RPMI without serum per $7.5 \times 10^{4}$ cells and allowed to complex for 20 minutes. The complex was then added to the media of the cells to be transfected. 4 hours later the media was replaced. In all experiments pcDNA-GFP was transfected as a control to assess transfection efficiency, which was checked via fluorescence imaging 24 hours after transfection.

\subsection{Luciferase Reporter Assay}

\subsubsection{Buffers}

Cell Culture Lysis Reagent (CCLR): $25 \mathrm{mM}$ tris-phosphate, $2 \mathrm{mM}$ dithiothreitol, 2 $\mathrm{mM}$ 1,2-diaminocyclohexane-N,N,N',N,-tetraacetic acid, 10\% (v/v) glycerol, 1\% (v/v) Triton X-100

\subsubsection{Treatment after Transfection}

After the 4 hour incubation period with the transfection reagents, plates were either irradiated with $10 \mathrm{~Gy}$ and the media replaced or the media was replaced with RPMI containing $3 \mu \mathrm{M}$ doxorubicin. Cells were harvested 48 hours after treatment. 


\subsubsection{Luciferase Reporter Assay}

The luciferase assay was measured using the Promega Luciferase Assay system. Forty-eight hours after transfection with $\mathrm{BCL6}_{4}$-tkLUC or PGL3 or pcDNA-GFP plasmid and treatment, each well was photographed on the Olympus IX51 microscope with the Olympus cellSens standard software at $10 \mathrm{x}$ magnification and cells were harvested. Media was removed and cells were washed in $1 \mathrm{ml}$ cold PBS. Three-hundred $\mu \mathrm{l}$ cell culture lysis reagent (CCLR) was added to each well before cells were lysed and lysate collected using the rubber bung of a $2 \mathrm{ml}$ syringe as a scraper. Twenty $\mu$ l of cell lysate of each sample was added to a 96 well solid white flat bottom plate. The luciferase assay reagent (LAR) was prepared according to manufacturers instructions. The plate was read with the Tecan Infinite M1000 Pro Plate Reader (Tecan, Switzerland), where the plate reader injected $100 \mu$ of LAR into a well and then took readings every 5 seconds for a total of 20 seconds. This process was repeated for all wells. Light values were first normalised to cell number as counted from the well photographs to give a "luciferase per cell" value. These values were then normalised to the untreated, single transfected control equivalent (either $\mathrm{BCL6}_{4}$-tkLUC or PGL3 plasmid) and plotted as a $\%$ relative activity for each treatment.

\subsection{Western Blot}

\subsubsection{Buffers}

Crude Lysis Buffer: $70 \mathrm{mM} \mathrm{NaCl}, 20 \mathrm{mM}$ tris, 0.1\% (v/v) IGEPAL

Lamelli Buffer: 20\% (v/v) glycerol, $15 \mathrm{mg} / \mathrm{ml}$ Tris-Cl ( $\mathrm{pH}$ 6.8), 0.01\% (w/v) bromophenol blue, $20 \mathrm{mM}$ dithiothreitol, $40 \mathrm{mg} / \mathrm{ml}$ SDS

SDS Running Buffer: $120 \mathrm{mM}$ glycine, $40 \mathrm{mM}$ tris, $0.1 \%(\mathrm{w} / \mathrm{v})$ sodium dodecyl sulphate

Transfer Buffer: $120 \mathrm{mM}$ glycine, $40 \mathrm{mM}$ tris, 10/20\% (v/v) methanol

Ponceau Red: 0.2\% (w/v) Ponceau S, 3\% (v/v) acetic acid

PBS-T: $137 \mathrm{mM} \mathrm{NaCl}, 2.68 \mathrm{mM} \mathrm{KCl}, 10.1 \mathrm{mM} \mathrm{Na}_{2} \mathrm{HPO}_{4}, 1.76 \mathrm{mM} \mathrm{KH}_{2} \mathrm{PO}_{4}, 0.1 \%$ (v/v) Tween-20 


\subsubsection{Gel composition}

6\% resolving gel: $454 \mathrm{mM}$ Tris- $\mathrm{Cl}(\mathrm{pH} 8.8), 347 \mathrm{mM} \mathrm{SDS}, 6 \%(\mathrm{v} / \mathrm{v})$ acrylamide solution, $2.2 \mathrm{mM}$ ammonium persulphate, $0.05 \%(\mathrm{v} / \mathrm{v})$ tetramethylethylenediamine 4\% stacking gel: $125 \mathrm{mM}$ Tris- $\mathrm{Cl}(\mathrm{pH}$ 6.8), $6.9 \mathrm{mM} \mathrm{SDS,} \mathrm{4 \% (v/v)} \mathrm{acrylamide}$ solution, $2.2 \mathrm{mM}$ ammonium persulphate, $0.05 \%(\mathrm{v} / \mathrm{v})$ tetramethylethylenediamine

\subsubsection{Antibodies}

\begin{tabular}{|c|c|c|c|}
\hline Antibody & Supplier & & Concentration \\
\hline Goat anti-rabbit HRP & $\begin{array}{l}\text { Santa Cruz } \\
\text { USA }\end{array}$ & Biotechnology, & $1: 7000$ \\
\hline Mouse monoclonal anti-Actin & Sigma-Aldrich, & NZ & $1: 1000$ \\
\hline Mouse monoclonal anti-BCL6 (D8) & $\begin{array}{l}\text { Santa Cruz } \\
\text { USA }\end{array}$ & Biotechnology, & $1: 500$ \\
\hline Mouse monoclonal anti-BCOR & Sigma-Aldrich, & $\mathrm{NZ}$ & $1: 1000$ \\
\hline $\begin{array}{l}\text { Mouse monoclonal anti-NCOR2 } \\
\text { (SMRT) }\end{array}$ & Sigma-Aldrich, & NZ & $1: 1000$ \\
\hline Mouse monoclonal anti- $\alpha$ tubulin & Sigma-Aldrich, & NZ & $1: 1000$ \\
\hline Rabbit anti-mouse HRP & $\begin{array}{l}\text { Santa Cruz } \\
\text { USA }\end{array}$ & Biotechnology, & $1: 7000$ \\
\hline $\begin{array}{l}\text { Rabbit polyclonal anti-NCOR1 } \\
\text { (ab24552) }\end{array}$ & Abcam, UK & & $1: 1000$ \\
\hline
\end{tabular}

\subsubsection{Protein Extraction}

Media was removed from cells and they were scraped off the tissue culture surface into cold Dulbecco's PBS (DPBS). Cells were pelleted in a microcentrifuge and the supernatant removed. They were then resuspended in 40-500 $\mu$ l Crude Lysis Buffer supplemented with complete protease inhibitor. The cells were left to lyse on ice for an hour, before centrifugation at top speed for 10 minutes. The supernatant was kept and a small amount was used for protein quantification. The remaining supernatant was heated in Laemelli Buffer added to a $1 \mathrm{x}$ concentration at $95^{\circ} \mathrm{C}$ for 5 minutes. 


\subsubsection{Protein Quantification}

Protein was quantified by Qubit ${ }^{\mathrm{TM}}$ fluorometer as detailed in 2.4.3.

\subsubsection{SDS-Acrylamide Gel Preparation}

The plates of $1.5 \mathrm{~mm}$ gel cassettes were cleaned thoroughly with Kimwipes ${ }^{\circledR}$ and $\mathrm{ddH}_{2} \mathrm{O}$ followed by $100 \%$ isopropanol (2-Propanol) to remove any residues from previous use. The plates were installed into cassette holders and the bottom sealed with Parafilm ${ }^{\circledR}$ before they were installed into the gel casting rack. The resolving gel solution was prepared (2.7.2) and poured into the prepared gel cassettes, with room left for the stacking gel to be poured. 100\% isopropanol was carefully poured on top of the gel solution in the cassette to remove bubbles and the gel was left for approximately 1 hour to set. Once set, a stacking gel solution was prepared (2.7.2) and isopropanol removed from the top of the resolving gel. The stacking gel was then poured on top of the resolving gel to fill the cassette, and a clean $1.5 \mathrm{~mm}$ comb was carefully inserted into the top of the gel cassette. The stacking gel was then left to set for approximately 1 hour.

\subsubsection{SDS- Polyacrylamide Gel Electrophoresis}

The gel cassettes were loaded into a BioRad mini PROTEAN® 3 cell gel tank and the tank was filled with chilled SDS Running Buffer. The combs of the gels were carefully removed to ensure the wells were straight. $5 \mu$ l of PageRuler ${ }^{\mathrm{TM}}$ Plus prestained protein ladder (Thermo Fisher, NZ) was loaded in lane 1, followed by 50 $\mu \mathrm{g}$ of sample protein per well. Once all samples were loaded, the gel was run at 160 $\mathrm{V}$ for 1 hour, or until sufficient separation was seen, using a Bio-Rad Powerpac 300.

\subsubsection{Western Blot}

Once the gel was run, PVDF membrane was cut to the correct size and hydrated in $100 \%$ methanol. The transfer cassette was then assembled while submerged in cold 
Transfer Buffer. A sponge soaked in transfer buffer was first placed on the plastic cassette, followed by filter paper, then the gel, then the PVDF membrane. At this point all bubbles were removed by using a roller to gently roll out any bubbles between the gel and membrane. The transfer cassette was completed by another piece of filter paper, followed by a transfer buffer soaked sponge. The transfer cassette was then placed into a BioRad mini PROTEAN® 3 cell gel tank and run at $300 \mathrm{~mA}$ for 2 hours at $4{ }^{\circ} \mathrm{C}$ for blotting BCOR and BCL6, or at $20 \mathrm{~V}$ for 16 hours at $4{ }^{\circ} \mathrm{C}$ for blotting NCOR1 and NCOR2 (SMRT). Once the transfer was complete, the membrane was removed and stained with Ponceau Red to check for protein transfer. The stain was removed by washing with PBS-T for 5 minutes. Once the stain was removed, the membrane was blocked with 5\% (w/v) BSA in PBS-T for 1 hour at RT on a rocker. After the membrane was blocked, the primary antibody was diluted to the appropriate concentration (see 2.7.3) in blocking solution and incubated with the membrane on a rocker at $4^{\circ} \mathrm{C}$ overnight.

After overnight incubation, the blot was washed in PBS-T for 5 minutes 3 times. Once washed, the appropriate HRP conjugated secondary antibody was diluted 1:7000 and incubated with the blot for 1 hour at RT. After the secondary incubation, the blot was washed in PBS-T for 5 minutes 3 times.

Once washed, chemiluminescent detection of the bands was performed using the Western Lightning Ultra ECL kit (PerkinElmer, USA). The reagents were mixed 1:1 in a volume appropriate for the membrane size and pipetted onto the blot. Once even coverage of the blot was ensured, the blot was imaged using a CCD camera (Amersham Imager 600, GE Healthcare Life Sciences). After the blot was imaged it was then incubated with Restore Western Blot Stripping Buffer for 15 minutes at RT to remove primary and secondary antibodies. Once stripped, the membrane was blocked in BSA and the process repeated for all remaining proteins to be investigated. 


\subsection{Electrophoretic Mobility Shift Assay}

\subsubsection{Buffers}

Buffer A: 10 mM HEPES-KOH (pH 7.9), 1.5 mM MgCl, $10 \mathrm{mM} \mathrm{KCl,} 0.5 \mathrm{mM}$ dithiothreitol

Buffer B: 20 mM HEPES-KOH (pH 7.9), $420 \mathrm{mM} \mathrm{NaCl}, 1.5 \mathrm{mM} \mathrm{MgCl} 20.2 \mathrm{mM}$ EDTA, $0.5 \mathrm{mM}$ dithiothreitol

1x TBE: $80 \mathrm{mM}$ tris, $90 \mathrm{mM}$ boric acid, $2 \mathrm{mM}$ EDTA

Probe Buffer: $10 \mathrm{mM}$ tris-Cl, $10 \mathrm{mM} \mathrm{MgCl}, 50 \mathrm{mM} \mathrm{NaCl}, 1 \mathrm{mM}$ DT'T

Binding Buffer (10x): $100 \mathrm{mM}$ tris, $500 \mathrm{mM} \mathrm{KCl,} 10 \mathrm{mM}$ DTT

\subsubsection{Native gel composition}

$6 \%$ native gel: $0.5 \mathrm{x}$ TBE, $6 \%(\mathrm{v} / \mathrm{v})$ acrylamide solution, $2.2 \mathrm{mM}$ ammonium persulphate, $0.05 \%(\mathrm{v} / \mathrm{v})$ TEMED

\subsubsection{Probes}

\begin{tabular}{lcl}
\hline Top Strand & 5'-GAAAATTCCTAGAAAGCATA-3' & $\begin{array}{l}\text { Integrated DNA Technologies, } \\
\text { Singapore }\end{array}$ \\
Bottom Strand & 5'-TATGCTTTCTAGGAATTTTC-3' & $\begin{array}{l}\text { Integrated DNA Technologies, } \\
\text { Singapore }\end{array}$
\end{tabular}

Bold indicates BCL6 binding sequence, probes were biotinylated at the 3' end

\subsubsection{Antibodies}

\begin{tabular}{ll} 
Antibody & Supplier \\
\hline Goat polyclonal anti-BCOR (ab5276) & Abcam, UK \\
Mouse monoclonal anti-BCL6 (N3) & Santa Cruz Biotechnology, USA \\
Mouse monoclonal anti-SMRT & GeneTex, USA \\
Rabbit polyclonal anti-NCOR1 (ab24552) & Abcam, UK
\end{tabular}




\subsubsection{Cell preparation for EMSA}

Cells were transfected with wild-type BCL6 expression plasmid as a positive control (see 2.5.3) and harvested 24 hours after transfection.

\subsubsection{Protein Extraction}

Cells were washed with cold PBS and pelleted by centrifugation. Cells were resuspended in $400 \mu \mathrm{l}$ cold Buffer A and allowed to swell on ice for 10 minutes. Cells were then vortexed for 10 seconds and centrifuged briefly to pellet the nuclei. The pellet was resuspended in $100 \mu \mathrm{l}$ cold Buffer B and incubated on ice for 20 minutes. The insoluble material was pelleted by centrifugation and the supernatant kept on ice.

\subsubsection{Protein Quantification}

Due to Buffer B interacting with the Qubit ${ }^{\mathrm{TM}}$ Protein Assay, protein for EMSA was quantified using the BioRad protein assay, according to the manufacturers instructions. Briefly, 4 BSA standards at $0,0.25,0.5$ and $1 \mu \mathrm{g} / \mathrm{ml}$ were thawed while the Dye Reagent Concentrate was diluted 1:4 in $\mathrm{ddH}_{2} \mathrm{O}$. The protein samples were diluted 1:20 (final volume $40 \mu \mathrm{l}, 2 \mu \mathrm{l}$ of sample used) in $\mathrm{ddH}_{2} \mathrm{O}$ before $10 \mu \mathrm{l}$ of either standard or sample was pipetted in triplicate into a 96 well clear flat bottom plate. Once the sample was added, $200 \mu$ of the Dye Reagent was added to each well. The plate was incubated at RT for 5 minutes before the absorbance was read at $595 \mathrm{~nm}$. Once the plate was read, Microsoft Excel was used to calculate the equation of the line of the standards, which was then used to calculate the concentration of the samples.

\subsubsection{Native Acrylamide Gel Preparation}

The plates of $1.5 \mathrm{~mm}$ gel cassettes were cleaned thoroughly with Kimwipes ${ }^{\circledR}$ and $\mathrm{ddH}_{2} \mathrm{O}$ followed by $100 \%$ isopropanol (2-Propanol) to remove any residues from previous use. The plates were installed into cassette holders and the bottom sealed with Parafilm ${ }^{\circledR}$ before they were installed into the gel casting rack. The native gel 
solution was prepared (2.8.2) and poured into the prepared gel cassettes. Once filled, a clean $1.5 \mathrm{~mm}$ comb was carefully inserted into the top of the gel cassette. The gel was then left to set for approximately 1 hour. If the gel was stored for future use, the gel cassette was then wrapped in a damp paper towel and placed in a plastic bag with a small amount of $0.5 \mathrm{x}$ TBE in the cold room for up to a week before use.

\subsubsection{Labelled Probe and Unlabelled Competitor DNA Preparation}

Labelled probe was prepared by dilution of both the top and bottom strands to a concentration of $0.01 \mu \mathrm{M}$ in a total volume of $50 \mu \mathrm{l}$ Probe Buffer. Unlabelled competitor was prepared by dilution of bot the top and bottom strands to a concentration of $1 \mu \mathrm{M}$ in a total volume of $50 \mu \mathrm{l}$ Probe Buffer. Both solutions were heated to $95{ }^{\circ} \mathrm{C}$ to fully denature the strands and allowed to cool to RT in the heat block for the probes to anneal.

\subsubsection{Antibody and Probe Binding}

The LightShift Chemiluminescent EMSA kit was used for the binding reactions and detection of biotin-labelled DNA. For all binding reactions, $5 \mu \mathrm{g}$ of nuclear extract was incubated with $20 \mathrm{fmol}$ of biotin-labelled probe, $50 \mathrm{ng} / \mu \mathrm{l}$ poly-dIdC (in $10 \mathrm{mM}$ tris and $1 \mathrm{mM}$ EDTA, pH7.5), 2.5\% (v/v) glycerol, $5 \mathrm{mM} \mathrm{MgCl}_{2}, 0.05 \%$ (v/v) NP40 and $2 \mu \mathrm{l}$ of 10x Binding Buffer in a total volume of $20 \mu \mathrm{l}$ for 20 minutes at RT. For the competition assay, 4 pmol of unlabelled competitor DNA was added to this reaction. For the supershift assay, $2 \mu \mathrm{g}$ of antibody was added.

\subsubsection{Gel Electrophoresis and Transfer}

The gel cassettes were loaded into a BioRad mini PROTEAN® 3 cell gel tank and the tank was filled with chilled $0.5 \mathrm{x}$ TBE. The combs of the gels were carefully removed to ensure the wells were straight. Once the wells were checked, $2 \mu \mathrm{l}$ of 100 bp DNA ladder was loaded in lane 1, EMSA samples were mixed with $2 \mu$ EMSA loading dye and $20 \mu \mathrm{l}$ of each sample was loaded in the following wells. Once all samples were loaded, the gel was run at $100 \mathrm{~V}$ for 60 minutes, or until sufficient 
separation was seen, using a Bio-Rad Powerpac 300. While the gel ran, nylon membrane cut to the correct size was soaked in 0.5x TBE. Once the gel had finished, it was sandwiched with the nylon membrane between two clean transfer sponges (not previously used for western blotting) and clean filter paper in cold $0.5 \mathrm{x}$ TBE. Transfer was performed in a clean transfer apparatus at $4{ }^{\circ} \mathrm{C}$ in $0.5 \mathrm{x}$ TBE, at $380 \mathrm{~mA}$ for 40 minutes.

\subsubsection{Cross-linking and Detection of Biotin-labeled DNA}

After transfer, the membrane was carefully removed and allowed to dry briefly on a paper towel. The membrane was placed face down on a transilluminator set to "high, $302 \mathrm{~nm}$ " for 15 minutes to cross-link the DNA to the membrane. While the membrane was being cross-linked, the 4x Wash Buffer and Blocking Buffer components of the Lightshift Chemiluminescent EMSA Kit were heated to $37^{\circ} \mathrm{C}$ or until all particulate dissolved into solution. After the membrane was cross-linked, it was blocked by incubation in $20 \mathrm{ml}$ Blocking Buffer for 15 minutes with gentle shaking at RT. While it was being blocked, Conjugate/Blocking Buffer was prepared by the addition of $66.7 \mu$ of stabilised strepdavidin-horseradish peroxidase conjugate to $20 \mathrm{ml}$ Blocking Buffer. After the membrane was blocked, the Blocking Buffer was removed and replaced with Conjugate/Blocking Buffer, which was incubated for 15 minutes with gentle shaking at RT. Wash buffer was diluted to a $1 \mathrm{x}$ concentration, and once incubation with Conjugate/Blocking Blocking Buffer was complete, the membrane was washed 4 times for 5 minutes with $20 \mathrm{ml}$ Wash Buffer and gentle shaking. After the wash steps, the membrane was incubated with $30 \mathrm{ml}$ Substrate Equilibrium Buffer for 5 minutes at RT with gentle shaking. For imaging the membrane, Substrate Working Solution was prepared by the addition of $3 \mathrm{ml}$ Luminol/Enhancer solution to $3 \mathrm{ml}$ Stable Peroxide solution, and the membrane was incubated with this solution for 5 minutes with no shaking. The membrane was then imaged with a CCD camera (Amersham Imager 600, GE Healthcare Life Sciences). 


\subsection{Chromatin Immunoprecipitation}

\subsubsection{Buffers}

Szak RIPA Buffer: $50 \mathrm{mM} \mathrm{NaCl}, 1 \%$ (v/v) IGEPAL, 0.5\% (w/v) Na-DOC, 0.1\% (w/v) SDS, 50 mM tris-Cl (pH 8.0), 5 mM EDTA (pH 8.0)

Swelling Buffer: 50 mM Hepes-KOH (pH 7.5), $140 \mathrm{mM} \mathrm{NaCl}, 10 \%$ glycerol, $1 \mathrm{mM}$ EDTA (pH 8.0), 0.5\% (v/v) IGEPAL, 0.25\% (v/v) Triton X-100

Washing Buffer: 10 mM tris-Cl (pH 8.0), 200 mM NaCl, 1 mM EDTA (pH 8.0), 0.5 mM EGTA (pH 8.0)

Shearing Buffer: 50 mM tris-Cl (pH 8.1), 0.1\% (w/v) SDS, 10 mM EDTA (pH 8.0)

Dilution Buffer: $300 \mathrm{mM} \mathrm{NaCl}, 2 \%$ (v/v) IGEPAL, 1\% (w/v) Na-DOC, $0.1 \%$ (v/v) SDS, $50 \mathrm{mM}$ tris-Cl (pH 8.0)

1x TAE: $40 \mathrm{mM}$ tris, $2 \mathrm{mM}$ EDTA (pH 8.0), $20 \mathrm{mM}$ Acetic Acid

6x Orange G loading dye: $10 \mathrm{mM}$ tris- $\mathrm{HCl}(\mathrm{pH} 7.6$ ), 0.15\% (w/v) orange $\mathrm{G}, 60 \%$ (v/v) glycerol, $60 \mathrm{mM} \mathrm{EDTA}$

Elution Buffer: 1\% (w/v) SDS, $100 \mathrm{mM} \mathrm{NaHCO}$

Low Salt Wash Buffer: $150 \mathrm{mM} \mathrm{NaCl}, 0.5 \%$ (w/v) Na-DOC, 0.1\% (w/v) SDS, 1\% (v/v) IGEPAL, 1 mM EDTA (pH 8.0), 50 mM tris-Cl (pH 8.0)

High Salt Wash Buffer: $500 \mathrm{mM} \mathrm{NaCl}, 0.5 \%$ (w/v) Na-DOC, 0.1\% (w/v) SDS, 1\% (v/v) IGEPAL, 1 mM EDTA (pH 8.0), 50 mM tris-Cl (pH 8.0)

Lithium Chloride Wash Buffer: 250mM LiCl, 0.5\% (w/v) Na-DOC, 0.1\% (w/v) SDS, $1 \%$ (v/v) IGEPAL, 1 mM EDTA (pH 8.0), 50 mM tris-Cl (pH 8.0)

TE: 0.25 mM EDTA (pH 8.0), $10 \mathrm{mM}$ tris- $\mathrm{Cl}$ (pH 8.0)

\subsubsection{Antibodies}

Antibody Supplier

$\begin{array}{ll}\text { Mouse monoclonal anti-RNA Polymerase II } & \text { EMD-Millipore, USA } \\ \text { Mouse monoclonal anti- } \beta \text {-actin } & \text { Sigma-Aldrich, NZ } \\ \text { Rabbit IgG } & \text { Santa Cruz Biotechnology, USA } \\ \text { Rabbit polyclonal anti-BCL6 (N3) } & \text { Santa Cruz Biotechnology, USA }\end{array}$




\subsubsection{Cell Culture and Treatment}

LN18 were plated at $1 \times 10^{7}$ per $150 \mathrm{~mm}$ dish or $4 \times 10^{6}$ per $100 \mathrm{~mm}$ dish and allowed to adhere for 24 hours. They were then treated with $3 \mu \mathrm{M}$ doxorubicin or 10Gy radiation or left untreated. Treated cells were harvested 8 or 48 hours after treatment, untreated cells were harvested 72 hours after plating.

\subsubsection{Agarose Bead Preparation (Melnick Lab)}

Protein A agarose beads were used for all ChIP experiments conducted in the Melnick lab. In advance of the ChIP procedure, $1 \mathrm{ml}$ of beads was centrifuged at 10,000 rpm for 10 minutes at $4{ }^{\circ} \mathrm{C}$ and supernatant discarded. The beads were washed by rocking with $1 \mathrm{ml}$ Szak RIPA Buffer for 5 minutes at $4{ }^{\circ} \mathrm{C}$, followed by centrifugation at $10,000 \mathrm{rpm}$ for 10 minutes at $4{ }^{\circ} \mathrm{C}$ and the supernatant was discarded. The beads were then resuspended in $1 \mathrm{ml}$ Szak RIPA Buffer with 1 $\mathrm{mg} / \mathrm{ml} \mathrm{BSA}$ and $1 \mathrm{x}$ protease inhibitor, and left to rock overnight at $4{ }^{\circ} \mathrm{C}$. After the rocking overnight, the beads were centrifuged at $10,000 \mathrm{rpm}$ for 10 minutes at $4{ }^{\circ} \mathrm{C}$ and supernatant discarded. The beads were then washed by rocking with $1 \mathrm{ml}$ Szak RIPA Buffer for 5 minutes at $4{ }^{\circ} \mathrm{C}$, repeated 3 times. After the final wash, Szak RIPA Buffer was added to a final volume of $1 \mathrm{ml}$ and the beads were ready for use.

\subsubsection{Dynabead® Preparation (McConnell Lab)}

Protein G Dynabeads ${ }^{\circledR}$ were used for all ChIP experiments conducted in the McConnell lab with $1 \mathrm{mg}$ of Protein G Dynabeads ${ }^{\circledR}$ used per antibody. The beads were placed on a magnet and the supernatant was removed. The beads were then washed in Szak RIPA Buffer twice, before being resuspended in $40 \mu$ of Szak RIPA Buffer per antibody. $5 \mu \mathrm{g}$ of antibody (either BCL6 (N3), RNA Polymerase II or $\beta$ actin) was added to the beads and they were rotated at $4{ }^{\circ} \mathrm{C}$ while the cells were prepared for immunoprecipitation. 


\subsubsection{Cell Fixation, Harvest and Nuclear Preparation}

For fixation, 37\% Formaldehyde was added to each plate to a final concentration of $1 \%$ and rocked for 10 minutes at RT. For quenching, glycine was added to a final concentration of $0.125 \mathrm{M}$ and rocked for 10 minutes at RT. Plates were then washed twice with cold PBS and scraped into cold PBS. Cells were collected into one tube per treatment and were pelleted by centrifugation at $500 \mathrm{xg}$ for 10 minutes at $4^{\circ} \mathrm{C}$. For nuclear preparation, cells were gently resuspended in $1 \mathrm{ml}$ Swelling Buffer supplemented with protease inhibitors per sample and rotated for 10 minutes at $4^{\circ} \mathrm{C}$. Nuclei were pelleted by centrifugation at $1700 \mathrm{xg}$ for 5 minutes at $4{ }^{\circ} \mathrm{C}$, and supernatant was carefully aspirated to ensure nuclei were not disturbed. Nuclei were gently resuspended in $1 \mathrm{ml}$ Wash Buffer supplemented with protease inhibitor per sample and rotated for 10 minutes at $4^{\circ} \mathrm{C}$. Nuclei were pelleted by centrifugation at $1700 \mathrm{~g}$ for 5 minutes at $4^{\circ} \mathrm{C}$, and supernatant was carefully aspirated to ensure nuclei were not disturbed.

\subsubsection{Sonication (Melnick Lab)}

Shearing Buffer supplemented with protease inhibitor was carefully added to the tube without disturbing the nuclei and then aspirated. Nuclei were resuspended in $975 \mu$ l Shearing Buffer supplemented with protease inhibitors and transferred to a 1 $\mathrm{ml}$ AFA millitube. Each tube was added to the 24 well plate of the Covaris E220 ultrasonicator and sonicated for 20 minutes per tube with the following conditions: $200 \mathrm{cpb}, 5 \% \mathrm{DF}, 140 \mathrm{~W}$, water level 1-5 mm below tube cap. Once sonicated, sonication efficiency was checked. $20 \mu \mathrm{l}$ of the sonicated sample was taken and $20 \mu \mathrm{l}$ of Elution Buffer with $0.3 \mathrm{M} \mathrm{NaCl}$ was added. The sample was heated to $95^{\circ} \mathrm{C}$ for 5 minutes before DNA extraction with Qiagen minElute PCR purification kit. DNA extraction was completed according to the manufacturers instructions. Briefly, sample was diluted in $200 \mu \mathrm{l}$ buffer PB and added to the column. The column was centrifuged at 13,000 $\mathrm{g}$ for 1 minute, the flow though was discarded and $750 \mu \mathrm{l}$ buffer PE was added to the column. The column was centrifuged at 13,000 xg for 1 minute, the flow through discarded and the column was centrifuged again for another minute to remove remaining buffer. $15 \mu \mathrm{l}$ of buffer EB was added to the 
column and it was left to stand for 5 minutes at RT. The column was then centrifuged at $13000 \mathrm{xg}$ for 1 minute to elute DNA.

\subsubsection{Sonication (McConnell Lab)}

As in the Melnick lab, Shearing Buffer supplemented with protease inhibitor was carefully added to the tube without disturbing the nuclei and then aspirated. Nuclei were resuspended in Shearing Buffer supplemented with protease inhibitors and sonicated on ice $(50 \%$ amplitude, $20 \mathrm{~s}$ pulse, $20 \mathrm{~s}$ rest, for 20 minutes on a SONOPULS mini20 ultrasonic homogeniser). Once sonicated, sonication efficiency was checked using the same protocol and kit as used in the Melnick lab.

\subsubsection{Gel Preparation and Sample Analysis}

For a 1\% agarose gel, $0.3 \mathrm{~g}$ of agarose was mixed with $30 \mathrm{ml}$ of $1 \mathrm{x}$ TAE Buffer and microwaved until completely dissolved. $10 \mu \mathrm{l}$ of Sybr ${ }^{\circledR}$ Safe was added to the gel before pouring. Once the gel was set, samples were prepared by the addition of $3 \mu \mathrm{l}$ of 6x Orange $\mathrm{G}$ loading dye to the $15 \mu \mathrm{l}$ of sample before loading the entire sample into the well. $100 \mathrm{bp}$ marker was used as a guide for fragment size. The gel was run at $100 \mathrm{~V}$ for 40 minutes in $1 \mathrm{x}$ TAE buffer. Once the run was complete, the gel was visualised under UV light on either the ChemiDoc MP (Melnick Lab) or on a transilluminator and images were captured using Point Grey software (McConnell Lab).

\subsubsection{Preclearing and Immunoprecipitation (Melnick Lab)}

Once sonication efficiency was determined, and before addition of antibody, the chromatin sample was precleared with the Protein A agarose beads. The chromatin sample was diluted 1:1 in Dilution Buffer and $40 \mu \mathrm{l}$ of blocked beads was added per $1 \mathrm{ml}$ of chromatin sample. The samples were then rocked at $4{ }^{\circ} \mathrm{C}$ for 2 hours. The samples were centrifuged at $4000 \mathrm{xg}$ for 1 minute at $4{ }^{\circ} \mathrm{C}$ and the supernatant was removed to be incubated with antibody overnight. An aliquot of $100 \mu \mathrm{l}$ of the chromatin sample was taken for input (10\% input sample), and the remainder of the chromatin was sample separated into $1 \mathrm{ml}$ aliquots (equivalent of $1 \times 10^{7}$ cells). For 
ChIP, $5 \mu \mathrm{g}$ of each antibody (either BCL6(N3) or Rabbit IgG) was added per $1 \mathrm{ml}$ chromatin sample and rocked overnight at $4{ }^{\circ} \mathrm{C}$. After overnight rocking, $30 \mu \mathrm{l}$ of blocked Protein A agarose beads were added to each tube and the samples were rocked for 2 hours at $4{ }^{\circ} \mathrm{C}$. After rocking the samples were centrifuged at $4000 \mathrm{xg}$ for 1 minute at $4{ }^{\circ} \mathrm{C}$, the supernatant discarded and proceeded with washes.

\subsubsection{Immunoprecipitation (McConnell Lab)}

Once sonication efficiency was determined, samples were divided to give the equivalent of $1 \times 10^{7}$ cells per target antibody. Samples were then diluted 1:1 in Dilution Buffer and the antibody/Dynabead ${ }^{\circledR}$ combination prepared in 2.9.5 was added to the appropriate sample. Samples were then rotated overnight at $4{ }^{\circ} \mathrm{C}$.

\subsubsection{Washes (Melnick Lab)}

Beads were washed in $1 \mathrm{ml}$ Szak RIPA Buffer and rotated at $4{ }^{\circ} \mathrm{C}$ for 5 minutes. Beads were then centrifuged at $4000 \mathrm{~g}$ for 5 minutes at $4{ }^{\circ} \mathrm{C}$. The supernatant was removed, and $1 \mathrm{ml}$ of cold Low Salt Wash Buffer was added. Beads were rocked for 5 minutes at $4{ }^{\circ} \mathrm{C}$, and were then centrifuged at $4000 \mathrm{xg}$ for 5 minutes at $4{ }^{\circ} \mathrm{C}$ and the supernatant was removed. This step was repeated once for a total of two washes with Low Salt Wash Buffer. Once this step was repeated, $1 \mathrm{ml}$ of cold High Salt Wash Buffer was added to each sample. Beads were rocked for 5 minutes at $4{ }^{\circ} \mathrm{C}$, and were then centrifuged at $4000 \mathrm{xg}$ for 5 minutes at $4{ }^{\circ} \mathrm{C}$ and the supernatant was removed. This step was repeated once for a total of two washes with High Salt Wash Buffer. Once this step was repeated, $1 \mathrm{ml}$ of cold Lithium Chloride Wash Buffer was added to each sample. Beads were rocked for 5 minutes at $4{ }^{\circ} \mathrm{C}$, and were then centrifuged at $4000 \mathrm{xg}$ for 5 minutes at $4{ }^{\circ} \mathrm{C}$ and the supernatant was removed. This step was repeated once for a total of two washes with Lithium Chloride Wash Buffer. Once this step was repeated, $1 \mathrm{ml}$ of cold TE was added to each sample. Beads were rocked for 5 minutes at $4{ }^{\circ} \mathrm{C}$, and were then centrifuged at $4000 \mathrm{xg}$ for 5 minutes at $4{ }^{\circ} \mathrm{C}$ and the supernatant was removed. This step was repeated once for a total of two washes with TE. 


\subsubsection{Washes (McConnell Lab)}

After overnight incubation with the antibody/beads, sample tubes were placed on a magnet and the supernatant removed. Beads were then washed with Szak RIPA Buffer, Low Salt Wash Buffer, High Salt Wash Buffer, Lithium Chloride Wash Buffer and TE as detailed in Washes (Melnick Lab), with a magnet used instead of centrifugation.

\subsubsection{Reverse Cross-links and DNA Extraction}

Once the wash steps were completed, $100 \mu \mathrm{l}$ of Elution Buffer was added to the beads. The beads were then vortexed for 30 seconds and heated at $65^{\circ} \mathrm{C}$ for 1 hour. Once heated, the sample tubes were placed on a magnet and supernatant was removed and put into a fresh tube, the beads discarded. $\mathrm{NaCl}$ was added to a final concentration of $300 \mathrm{mM}$ to the supernatants and $10 \%$ input sample. All samples were then heated to $65^{\circ} \mathrm{C}$ overnight. After overnight incubation, DNA extraction was performed with the Qiagen PCR purification kit, according to the manufacturers instructions. Briefly, sample was diluted in $500 \mu \mathrm{l}$ buffer PB and added to the column. The column was centrifuged at 13,000 $\mathrm{xg}$ for 1 minute, the flow though was discarded and $750 \mu \mathrm{l}$ buffer PE was added to the column. The column was centrifuged at 13,000 $\mathrm{xg}$ for 1 minute, the flow through discarded and the column was centrifuged again for another minute to remove remaining buffer. $100 \mu$ of buffer EB was added to the column and it was left to stand for 5 minutes at RT. The column was then centrifuged at 13,000 xg for 1 minute to elute DNA. qPCR was then performed as detailed in $\mathbf{2 . 1 0 . 3}$ for genes BCL6 Exon 1, BCL6 Intron 9, TARS, CCND2 and ATR. 


\subsection{PCR}

\subsubsection{Primers}

\begin{tabular}{lll} 
& Forward & Reverse \\
\hline ATR & CAGGCTTGAGAACAGCAG & CAGGCTTGAGAACAGCAG \\
& AG & AG \\
BCL6 Exon 1 & GCAGTGGTAAAGTCCGAA & AGCAACAGCAATAATCACC \\
& GC & TG \\
BCL6 Intron & AACCTGCAAAACATGGTT & AATTTGCCCAAACAGCAA \\
9 & ATTT & GT \\
CCND2 & GTTGTTCTGGTCCCTTTAA & ACCTCGCATACCCAGAGAT \\
& TCG & AAA \\
TARS & TATCTACGGTGTCCGGGA & CCTACTCTCCGCTGACCTT \\
& AG & G \\
\hline
\end{tabular}

All primers for qChIP were purchased from Integrated DNA Technologies, Singapore

\section{Quantitect Primer Assays}

\begin{tabular}{ll}
\hline BCL6 & Qiagen, NZ \\
BCOR & Qiagen, NZ \\
HPRT1 & Qiagen, NZ \\
NCOR1 & Qiagen, NZ \\
NCOR2 (SMRT) & Qiagen, NZ \\
\hline
\end{tabular}

\subsubsection{Reverse Transcription to produce cDNA}

Reverse transcription was performed using the iScript cDNA synthesis kit according to the manufacturers instructions. Briefly, $250 \mathrm{ng}$ of RNA was diluted to a final volume of $15 \mu \mathrm{l}$ in a PCR tube followed by $4 \mu \mathrm{l}$ of $5 \mathrm{x}$ Reaction mix and $1 \mu \mathrm{l}$ iScript Reverse Transcriptase. The tube was centrifuged briefly to ensure the entire mixture was at the bottom of the tube. The tube was then placed in a thermocycler and the following program was run: 
1. $25{ }^{\circ} \mathrm{C} 5$ minutes (priming)

2. $42{ }^{\circ} \mathrm{C} 30$ minutes (reverse transcription)

3. $85^{\circ} \mathrm{C} 5$ minutes (inactivation of reverse transcriptase)

4. $\quad 4^{\circ} \mathrm{C}$ hold

Once the reaction had finished, the cDNA was transferred to a $1.5 \mathrm{ml}$ microcentrifuge tube and diluted to a final volume of $100 \mu \mathrm{l}$ before proceeding with qPCR.

\subsubsection{Quantitative PCR (qPCR)}

Quantitative PCR was performed in a $10 \mu \mathrm{l}$ reaction volume with the KAPA SYBR ${ }^{\circledR}$ FAST Universal One-Step qRT-PCR Kit in a 96 well $10 \mu \mathrm{l}$ qPCR plate. Each well contained $5 \mu \mathrm{l} 2 \mathrm{x}$ KAPA SYBR ${ }^{\circledR}$ FAST Master Mix, $0.4 \mu \mathrm{l}$ of forward and reverse primers (200 nM final conc), $0.2 \mu \mathrm{l} 50 \mathrm{x}$ ROX Low, $0.4 \mu \mathrm{l}$ DEPC water and $5 \mu$ l DNA.

For qChIP, the following protocol was used:

1. $95{ }^{\circ} \mathrm{C} 3$ minutes (activation of polymerase)

2. $95{ }^{\circ} \mathrm{C} 1$ second (denaturation)

3. $60{ }^{\circ} \mathrm{C} 20$ seconds (priming/extension)

Steps 2-3 were repeated 40 times

For q-RT-PCR with Qiagen primer assays, the following protocol was used:

1. $94{ }^{\circ} \mathrm{C} 15$ minutes (activation of polymerase)

2. $94{ }^{\circ} \mathrm{C} 15$ seconds (denaturation)

3. $55^{\circ} \mathrm{C} 30$ seconds (priming)

4. $72{ }^{\circ} \mathrm{C} 35$ seconds (extension)

Steps 2-4 were repeated 40 times.

For qChIP data fold change values were generated with the $\Delta \mathrm{Ct}$ method. Average Ct values for each gene of interest were subtracted from the $\mathrm{IgG}$ control to generate a $\Delta \mathrm{Ct}$. This $\Delta \mathrm{Ct}$ value was then put into the formula $-2^{\wedge}(\Delta \mathrm{Ct})$ to generate a fold change value. 
Fold change values for q-RT-PCR were calculated using the $\Delta \Delta \mathrm{Ct}$ method. Average Ct values for each gene of interest were subtracted from the HPRT housekeeping gene which gave a $\Delta \mathrm{Ct}$, and then subtracted from the untreated or DMSO control, as appropriate to give a $\Delta \Delta \mathrm{Ct}$. The $\Delta \Delta \mathrm{Ct}$ was then put into the formula $-2^{\wedge}(\Delta \Delta \mathrm{Ct})$ to give a fold change value.

\subsection{RNA Sequencing}

\subsubsection{Sample Preparation}

$1 \times 10^{6} \mathrm{LN} 18$ were plated on a $100 \mathrm{~cm}^{3}$ flask 24 hours before treatment. Cells were treated with either $3 \mu \mathrm{M}$ doxorubicin or $10 \mathrm{~Gy}$ radiation or left untreated and harvested 24 hours later. For harvest, cells were trypsinised and washed in DPBS before RNA extraction.

\subsubsection{RNA Extraction}

RNA was extracted using the Zymo Quick RNA MiniPrep kit according to the manufacturers instructions. Briefly, cells were suspended in $600 \mu \mathrm{l}$ RNA Lysis Buffer and added to a Spin-Away ${ }^{\mathrm{TM}}$ Filter to remove genomic DNA. The column was centrifuged at $13000 \mathrm{xg}$ for 1 minute. $600 \mu \mathrm{l}$ of $100 \%$ ethanol was added to the flow through, and then loaded onto the Zymo-Spin IIICG column. The column was centrifuged at $13000 \mathrm{xg}$ for 1 minute and the flow through discarded. $400 \mu \mathrm{l}$ of RNA Prep Buffer was added to the column and the column was centrifuged at 13,000 xg for 1 minute. The flow through was discarded and $700 \mu$ RNA Wash Buffer was added to the column. The column was centrifuged at 13,000 xg for 1 minute and the flow through discarded. $400 \mu$ of RNA was buffer was then added to the column and was centrifuged at 13,000 xg for 1 minute. The flow through was discarded and the column was centrifuged again at 13,000 $\mathrm{xg}$ to remove all remaining wash buffer. The column was transferred to a DNase/RNase free microtube and $50 \mu \mathrm{l}$ of DNase/RNase free water was added to the column. The column was incubated at RT for 5 minutes before it was centrifuged at 13,000 xg for 1 minute to elute the RNA. Eluted RNA was stored at $-80^{\circ} \mathrm{C}$. 


\subsubsection{RNA Quality Check}

The Qubit ${ }^{\mathrm{TM}}$ fluorometer was used to determine RNA concentration and the procedure detailed in 2.4.2 was used. For RNA purity the NanoDrop spectrophotometer was used to determine the 260/280 and 260/230 absorption ratio. A ratio of 2 indicates uncontaminated RNA. To determine the level of degradation of the RNA, the Agilent Bioanalyzer 2100 was used with the Agilent RNA 6000 Nano kit according to the manufacturers instructions. Reagents were allowed to equilibrate to RT for 30 minutes, before the gel matrix was prepared. To prepare the gel matrix, $550 \mu$ l of Agilent RNA 6000 Nano gel matrix was added to the top of the spin filter, which was then centrifuged at $1500 \mathrm{xg}$ for 10 minutes. Once the matrix was filtered, $65 \mu \mathrm{l}$ of matrix was mixed with $1 \mu \mathrm{l}$ of dye and vortexed to ensure it was properly mixed. The mixture was then centrifuged at $13000 \mathrm{xg}$ for 10 minutes. The chip to be loaded was removed from its packaging and installed in the chip priming station, and $9 \mu$ of gel-dye mix was added to the bottom of the well marked "G". To pressurise the chip, the syringe plunger was adjusted to $1 \mathrm{ml}$, before the chip priming station was closed. The plunger of the syringe was pushed down until it reached the clip and was held in this position for 30 seconds. The plunger was then released, and then pulled back into the $1 \mathrm{ml}$ position. A further $9 \mu$ of gel-dye mix was then pipetted into the top-right well and the well below it. The RNA 6000 Nano marker was then loaded onto the chip, with $5 \mu \mathrm{l}$ pipetted into the bottom of each of the wells not loaded with the gel-dye mix. Before loading, the RNA sample to be loaded was heat denatured in a water bath at $70{ }^{\circ} \mathrm{C}$ for 2 minutes. The chip was loaded with $1 \mu \mathrm{l}$ heat denatured RNA ladder in the well with the ladder symbol (bottom right) and $1 \mu$ l of sample or water was loaded into each of the 12 sample lanes. The chip was then vortexed for 60 seconds at $2400 \mathrm{rpm}$ before it was loaded into the 2100 Bioanalyzer. The Bioanalyzer program was run for the RNA 6000 Nano chip and the RNA Integrity Number (RIN) was used to determine the level of degradation of the RNA. The RIN has a range of 1-10, where 1 indicates highly degraded RNA and 10 indicates intact RNA. All RNA that was used for RNA sequencing had a RIN of 9.0 or above. 


\subsubsection{RNA Sequencing}

RNA was sent to New Zealand Genomics Limited (NZGL) for sequencing on the Illumina HiSeq platform, with TruSeq V2 stranded mRNA chemistry, run on a single lane. The average sequence length was $125 \mathrm{bp}$.

\subsubsection{RNA Sequencing Data Processing}

Processing of the raw data files, quality control, alignment, and quantification was completed by Samuel Lee, whose methods can be found at: https://doi.org/10.5281/zenodo.1022032

Briefly, FastQC was used to examine the quality of the raw sequencing reads, both before and after trimming (188). Trimmomatic (v0.36) (189) was used for the filtering and trimming of the RNA-seq reads in the fastq format prior to alignment. Illumina adapter sequences were removed, and all reads under 35 bp were discarded. Kallisto (v0.43.1) (190) was used for pseudoalignment and quantification of the sequence reads, against the Ensembl Human reference cDNA transcriptome release 89 (191). The individual transcript abundances produced by Kallisto were collapsed to the gene level using the R package tximport (v1.4.0) (192).

\subsubsection{RNA Sequencing Analysis}

Principal component, differential expression and gene set enrichment analyses were also performed by Samuel Lee.

DESeq2 (v1.16.1) (193) was used to perform principal component analysis (PCA) and differential expression analysis (DEA). Regular log transformation of count data was used for PCA. Condition comparisons for DEA were extracted using the Wald test, and reported p-values were then adjusted by the Benjamini-Hochberg method to correct for multiple testing. Gene set enrichment analysis (GSEA) was performed in Pre-Ranked mode (v3.0) with the classic enrichment statistic and 1,000 permutations. Gene lists to test were processed using $\mathrm{R}$ and gene-sets downloaded from MSigDB (v6.1) for local access before enrichment testing 
$(194,195)$. Unless otherwise stated, gene lists were curated by filtering transcript level DESeq2 results for an adjusted p-value (padj) $<0.05$ and a $\log 2$ fold change $(\log 2 \mathrm{fc})<-1.5$ and $>1.5$, and ranks calculated by the following formula: $\operatorname{sign}(\log 2 \mathrm{fc})$ $*_{-} \log 10$ (padj). 


\section{Expression and Activity of BCL6 in Glioblastoma}

\subsection{Introduction}

Glioblastoma is a disease that is remarkably difficult to treat. Standard treatment has barely changed in the past 30 years and offers limited extension of life after diagnosis $(20,85,111)$. Glioblastoma is highly resistant to treatment, and current treatments for GBM rely on the DNA damage response to induce death of GBM cells. In this study GBM cell lines LN18 and U87-MG were treated with the chemotherapeutic drug doxorubicin, or radiation, and BCL6 expression was investigated as a new potential mechanism of therapy resistance. BCL6 is known to function as a transcriptional repressor (196) and survival factor in response to DNA damage, specifically in the context of B-cell maturation (151). This function is important in the context of glioblastoma, as BCL6 may be the reason for GBM's resistance to DNA damage induced cell death. In order to determine if BCL6 is important in GBM, the first thing to examine is whether BCL6 is present and active in GBM. This has important implications for the treatment of GBM, as BCL6 inhibitors could be a promising mode of treatment for this notoriously difficult to treat disease.

Experiments in this thesis were only conducted in an in vitro setting, i.e. in cell lines, as opposed to an in vivo setting, i.e. in animal models. In vivo and in vitro models are fundamentally different experimental systems, which mean that drugs or other treatments that are fine to use in vivo may not work in the in vitro setting. Subsequently, in order to perform experiments in vitro that mimic treatments that occur in patients, adaptations must be made. For the experiments conducted in this thesis, a whole blood irradiator (Gammacell 3000 Elan, Theratronics, Ottawa, Canada) was used to irradiate the culture plates, irradiation was performed without media, and fresh warm media was put on the cells after irradiation. This is different to targeted treatment that is used on patients, which may not hit all GBM cells equally.

TMZ is the chemotherapeutic drug used to treat GBM patients in the clinic, however it was not used in the experiments conducted in this thesis. Experiments 
conducted before the commencement of this thesis (See Appendix: Fabre, Jones, et al., in revision) showed that BCL6 upregulation caused by TMZ dissolved in DMSO was difficult to distinguish from DMSO alone. TMZ is not particularly soluble in PBS, and other experiments done for this work found that when TMZ was dissolved in PBS, multiple doses were required to see an upregulation of BCL6. Consequently doxorubicin was chosen to be the chemotherapeutic used for this work instead as it is soluble in media and causes strong BCL6 upregulation with one dose, which meant it reduced the timeline for experiments and allowed for the extensive optimisation required for these techniques. Doxorubicin's primary mechanism of action is to intercalate DNA and inhibit topoisomerase II (96), which leads to breaks in the DNA that are difficult for the cell to repair, and when enough damage is done this results in cell death. Doxorubicin is used to treat a number of different cancers (90), but does not easily cross the blood-brain barrier alone so is not used to treat GBM, though there has been a number of approaches to formulate doxorubicin and deliver it across the blood brain barrier (91-94). Doxorubicin has a different mechanism of action to temozolomide, however the final result is the same, with both treatments leading to breaks in the DNA and subsequent cell death.

In order to function as a transcriptional repressor, BCL6 needs to recruit corepressors BCOR, NCOR, and SMRT $(157,197)$. These co-repressors are recruited to the BTB domain of BCL6 to form a complex that ultimately recruits HDAC3 to deacetylate the histone and shut down gene expression (158). It is this interaction with co-repressors that is targeted by most BCL6 inhibitors (198-200), which bind to this BTB site in BCL6 and prevent co-repressor recruitment.

The most recent, and most effective inhibitor of this interaction is called FX1, which was developed by the Melnick lab and first published in 2016 (179). FX1 has been shown to have high affinity for the BTB domain and prevent the recruitment of co-repressors. Lymphoma cells that are BCL6 dependent are sensitive to FX1, with an average $\mathrm{IC}_{50}$ of $36 \mu \mathrm{M}$, as compared with BCL6 independent lymphomas, which have an $\mathrm{IC}_{50}$ value of $>125 \mu \mathrm{M}$ (179). While FX1 is one of the most specific inhibitors of interaction with co-repressors to BCL6, there are limitations for its use. It has been shown that FX1 does inhibit non-target kinases at a concentration of 10 
$\mu \mathrm{M}$ (179), so it is possible that when used above this concentration, effects seen are not necessarily just related to BCL6 inhibition, and this effect may contribute to the $\mathrm{IC}_{50}$ value seen for BCL6 dependent lymphomas. Work in our lab on a panel of GBM cell lines has shown that GBM cells are similarly sensitive to FX1 treatment, with an $\mathrm{IC}_{50}$ of $40 \mu \mathrm{M}$ (See appendix: Fabre, Jones, et al., in revision) for LN18 cells. In this study FX1 was used to establish if BTB targeted inhibition affected the ability of BCL6 to function as a transcriptional repressor in GBM.

\subsection{Aims}

The aims of this chapter are to establish the presence of BCL6 and co-repressors BCOR, NCOR, and SMRT in GBM cells, and to determine if BCL6 is able to act as a transcriptional repressor when expressed in GBM cells.

\subsection{Results}

\subsubsection{Expression of BCL6 and co-repressors in glioblastoma cells LN18 and U87-MG}

To establish whether BCL6 and co-repressors BCOR, NCOR, and SMRT were expressed in GBM cells, mRNA was examined for BCL6, BCOR, NCOR, and SMRT transcripts in both LN18 and U87-MG cells under treated and untreated conditions. Cells were treated with $3 \mu \mathrm{M}$ doxorubicin, $10 \mathrm{~Gy}$ radiation or $25 \mu \mathrm{M}$ FX1 plus DMSO and untreated controls, and were harvested 48 hours after treatment. This long treatment time may mean that effects on transcript seen may be due to factors unrelated to treatment, such as different cells being at different stages of the cell cycle. When harvested, half of the sample was processed for RNA, the other half for protein. RNA was converted to cDNA and qPCR was run for BCL6, BCOR, NCOR, and SMRT, alongside the housekeeping gene HPRT. HPRT has been previously established in our lab as the least affected by cytotoxic treatment (201). Delta Ct $(\Delta \mathrm{Ct})$ values were calculated in relation to HPRT expression in each sample, and these have been plotted as $1 / \Delta \mathrm{Ct}$ to give a positive correlation with expression level. 
In Table 3.1 Ct values of qPCR for HPRT, BCL6, BCOR, NCOR, and SMRT are shown for LN18 cells. All of these values were below 30 cycles, which indicated transcript for all of these proteins was present regardless of treatment. In Figure 3.1 transcript of BCL6, BCOR, NCOR, and SMRT were measured in LN18 cells. Untreated LN18 cells did have detectable BCL6 transcript, which indicated that there was endogenous expression of BCL6 in LN18 cells. Doxorubicin treated cells had a higher transcript level of BCL6 as compared to untreated LN18 cells, which indicated that BCL6 expression is upregulated by doxorubicin treatment, even after 48 hours. Radiation treated cells had a similar level of BCL6 mRNA to the untreated cells, which indicated that radiation treatment did not affect BCL6 expression in LN18, but it is possible that transcript was increased at an earlier time point after radiation. FX1 treated cells also had a similar level of BCL6 mRNA to the DMSO treated cells, which indicated that FX1 did not have an effect additional to DMSO on BCL6 expression in LN18. DMSO control cells had a similar level of BCL6 transcript to the untreated cells, which indicated that DMSO did not have an effect on BCL6 expression in LN18. BCOR, NCOR, and SMRT expression were similar for doxorubicin, radiation, and FX1 treated cells, as compared to the untreated and DMSO controls, which indicated that co-repressor expression was not affected by these treatments.

RNA from K562 and Raji cells was harvested at the same time as LN18 cells to provide negative and positive controls for BCL6 expression. K562 is a chronic myelogenous leukaemia cell line that is BCL6 negative, and Raji is a Burkitt's lymphoma cell line that is BCL6 positive (202). In Figure 3.1 transcript of BCL6, BCOR, NCOR, and SMRT were measured in K562 and Raji cells. As expected, Raji cells had a much higher expression of BCL6 than K562 cells, but transcript for BCL6 was still detected in K562 cells. K562 appeared to have a much higher expression of BCOR than the Raji cells, and expression of NCOR and SMRT was similar between the two lines. 


\begin{tabular}{llllll} 
& Doxorubicin & Radiation & FX1 & DMSO & Untreated \\
\hline HPRT & $22.9 \pm 1.05$ & $21.2 \pm 0.36$ & $21.4 \pm 0.44$ & $22.0 \pm 0.22$ & $21.8 \pm 0.41$ \\
BCL6 & $28.0 \pm 0.95$ & $28.9 \pm 0.22$ & $29.4 \pm 0.80$ & $29.7 \pm 0.81$ & $29.8 \pm 0.85$ \\
BCOR & $28.0 \pm 1.63$ & $26.9 \pm 1.02$ & $28.4 \pm 0.39$ & $28.4 \pm 0.72$ & $27.9 \pm 1.06$ \\
NCOR & $27.3 \pm 1.63$ & $24.8 \pm 0.27$ & $25.6 \pm 0.21$ & $25.6 \pm 0.09$ & $25.5 \pm 0.07$ \\
SMRT & $28.2 \pm 2.02$ & $25.6 \pm 1.56$ & $26.9 \pm 1.43$ & $27.1 \pm 2.02$ & $26.6 \pm 1.46$
\end{tabular}

Table 3.1: Average Ct values for BCL6 and co-repressors in LN18 cells. LN18 cells were treated with either $3 \mu \mathrm{M}$ doxorubicin, $10 \mathrm{~Gy}$ irradiation, $25 \mu \mathrm{M}$ FX1, volume equivalent DMSO or left untreated and harvested 48 hours after treatment. Average $\mathrm{Ct}$ values are shown, \pm standard deviation, of at least 2 independent experiments. 


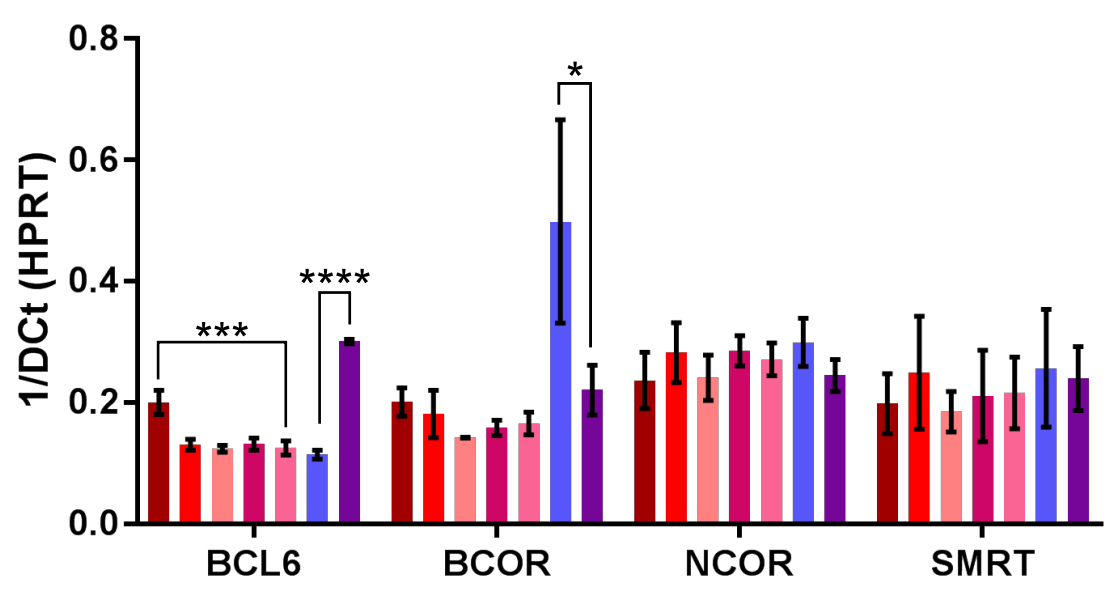

Figure 3.1: q-RT-PCR shows expression of BCL6 and co-repressors BCOR, NCOR, and SMRT in LN18 cells. LN18 cells were treated with either $3 \mu \mathrm{M}$ doxorubicin, $10 \mathrm{~Gy}$ irradiation, $25 \mu \mathrm{M}$ FX1, volume equivalent DMSO or left untreated and harvested 48 hours after treatment. K562 (BCL6 negative), and Raji (BCL6 positive) cells were harvested at the same time to serve as controls for BCL6 expression. Expression is shown as $1 / \mathrm{DCt}$, where DCt represents $\mathrm{Ct}$ value subtracted from the housekeeping gene HPRT. Bars represent the mean of at least 2 independent experiments and error bars represent \pm standard deviation. ${ }^{*} \mathrm{p}<0.05$, $* * * \mathrm{p}<0.005, * * * * \mathrm{p}<0.001$ Student's t-test. 
In Table 3.2 Ct values of qPCR for HPRT, BCL6, BCOR, NCOR, and SMRT are shown for U87-MG cells treated with doxorubicin, radiation, and FX1 with accompanying controls. Doxorubicin treatment had a significant effect on the mRNA of U87-MG, as housekeeping gene HPRT had a much higher Ct value (26.6 $\pm 0.89)$ as compared to the untreated control $(23.9 \pm 0.44)$. The Ct values for BCL6, BCOR, NCOR, and SMRT were all above 30 cycles in doxorubicin treated cells, and this low level of transcript can be explained by the doxorubicin effect seen on HPRT as compared to untreated cells. For all other treatment samples, Ct values were below 30 cycles, so we could be confident that transcript was present.

In Figure 3.2 transcript of BCL6, BCOR, NCOR, and SMRT was measured in U87-MG cells. Untreated U87-MG cells did appear to have a slightly higher level of BCL6 transcript as compared to K562 cells, which indicated that there was endogenous expression of BCL6 in U87-MG cells. Radiation treated cells had a similar level of mRNA to untreated cells, which indicated that BCL6 expression levels were not altered by radiation in U87-MG, but may have been altered at an earlier time point, as mentioned earlier. FX1 treated cells had a similar level of BCL6 mRNA to the DMSO treated cells, which indicated that FX1 did not have an effect on BCL6 expression additional to DMSO in U87-MG. DMSO control cells had a similar level of BCL6 expression as compared to untreated cells, which indicated that BCL6 expression was not affected by DMSO. BCOR, NCOR, and SMRT expression was similar between radiation and FX1 treated cells, as compared to the untreated and DMSO controls, which indicated that the expression of these co-repressors was not affected by these treatments. 


\begin{tabular}{llllll} 
& Doxorubicin & Radiation & FX1 & DMSO & Untreated \\
\hline HPRT & $26.6 \pm 0.89$ & $24.1 \pm 0.85$ & $23.5 \pm 1.34$ & $23.9 \pm 087$ & $23.9 \pm 0.44$ \\
BCL6 & $32.9 \pm 0.53$ & $29.4 \pm 1.22$ & $29.0 \pm 1.65$ & $30.3 \pm 1.14$ & $29.9 \pm 0.49$ \\
BCOR & $32.2 \pm 0.98$ & $29.6 \pm 1.57$ & $28.6 \pm 1.17$ & $29.1 \pm 0.84$ & $29.5 \pm 0.90$ \\
NCOR & $31.0 \pm 0.13$ & $27.9 \pm 1.38$ & $27.5 \pm 1.37$ & $27.8 \pm 1.01$ & $27.7 \pm 0.73$ \\
SMRT & $33.2 \pm 0.70$ & $27.8 \pm 0.64$ & $27.0 \pm 2.77$ & $27.8 \pm 2.09$ & $27.8 \pm 2.03$
\end{tabular}

Table 3.2: Average Ct values for BCL6 and co-repressors in U87-MG cells. U87MG cells were treated with either $3 \mu \mathrm{M}$ doxorubicin, $10 \mathrm{~Gy}$ irradiation, $25 \mu \mathrm{M}$ FX1, volume equivalent DMSO or left untreated and harvested 48 hours after treatment. Average $\mathrm{Ct}$ values are shown, \pm standard deviation, of at least 2 independent experiments. 


\section{Expression of BCL6 and \\ Co-Repressors in U87-MG}

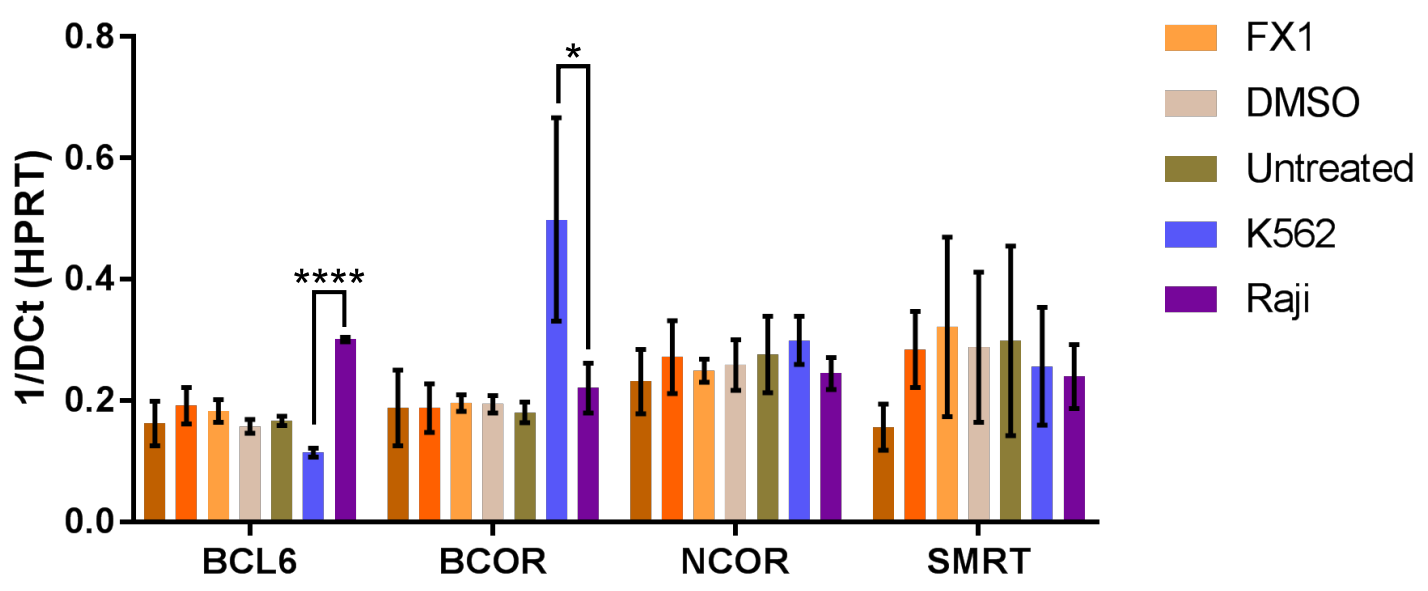

Figure 3.2: q-RT-PCR shows expression of BCL6 and co-repressors BCOR, NCOR, and SMRT in U87-MG cells. U87-MG cells were treated with either 3 $\mu \mathrm{M}$ doxorubicin, 10 Gy irradiation, $25 \mu \mathrm{M}$ FX1, volume equivalent DMSO or left untreated and harvested 48 hours after treatment. K562 (BCL6 negative) and Raji (BCL6 positive) cells were harvested at the same time to serve as controls for BCL6 expression. Expression is shown as $1 / \mathrm{DC}$, where DCt represents $\mathrm{Ct}$ value subtracted from the housekeeping gene HPRT. Bars represent the mean of at least 2 independent experiments and error bars represent \pm standard deviation. ${ }^{*} \mathrm{p}<0.05$, $* * * * \mathrm{p}<0.001$ Student's t-test. 
To see if transcript levels observed in LN18 and U87-MG cells reflected protein levels, BCL6, BCOR, NCOR, and SMRT proteins were assessed by western blot. The other half of the same samples harvested for RNA in Figure 3.1 and Figure 3.2 were processed for protein and blotted for BCL6, BCOR, NCOR, and SMRT as detailed in Section 2.7. Both $\alpha$-tubulin and $\beta$-actin proteins were used as loading controls due to potential differences in expression between the adherent GBM cell lines and the non-adherent K562 and Raji cells and the potential for alteration in protein level with drug treatment.

BCL6 protein was observed in all LN18 samples, with an increase in expression in cells treated with doxorubicin (Figure 3.3). These results were consistent with the RNA levels of BCL6 shown in Figure 3.1, and indicated that doxorubicin treatment lead to increased expression of BCL6 transcript and protein in LN18 cells.

BCL6 protein was observed in U87-MG cells treated with radiation, FX1 and DMSO, and untreated cells (Figure 3.4). However, unlike LN18, BCL6 protein was not observed in U87-MG cells treated with doxorubicin. This result was observed in 3 independent experiments and was consistent with the transcript levels of BCL6 (Figure 3.2) and indicated that BCL6 was not expressed and possibly downregulated in U87-MG cells in response to doxorubicin. 

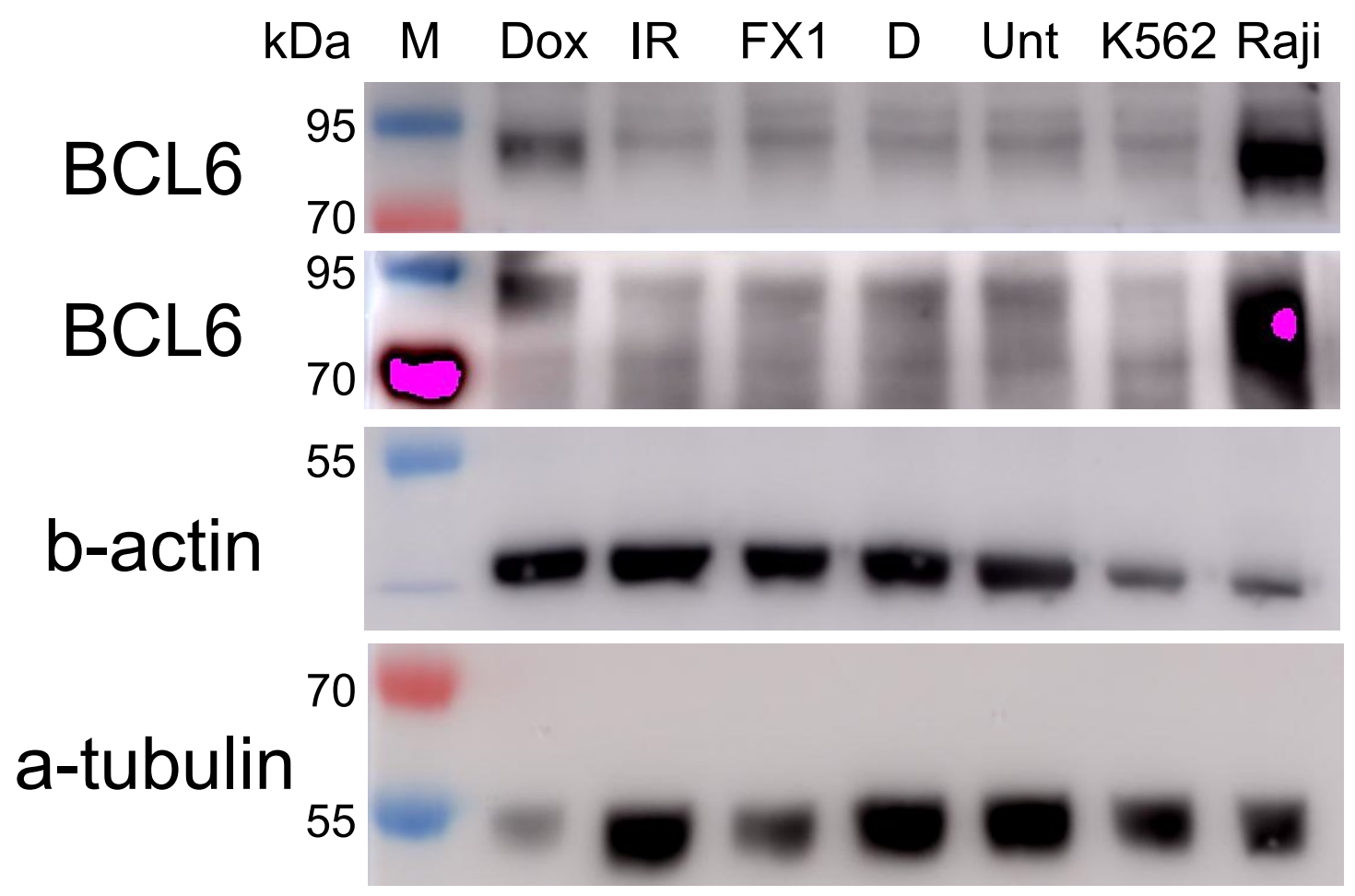

Figure 3.3: BCL6 is up-regulated in response to doxorubicin in LN18 cells. Western Blot was performed for BCL6, $\beta$-actin and $\alpha$-tubulin. LN18 cells were treated with either $3 \mu \mathrm{M}$ doxorubicin (Dox), 10 Gy irradiation (IR), $25 \mu \mathrm{M}$ FX1 (FX1), volume equivalent DMSO (D) or left untreated (Unt) and harvested for protein 48 hours after treatment. K562 (BCL6 negative) and Raji (BCL6 positive) cells were harvested at the same time to serve as controls for BCL6 expression. Both $\beta$-actin and $\alpha$-tubulin are shown as loading controls. M indicates size marker. Blot shown is representative of 3 independent experiments. 

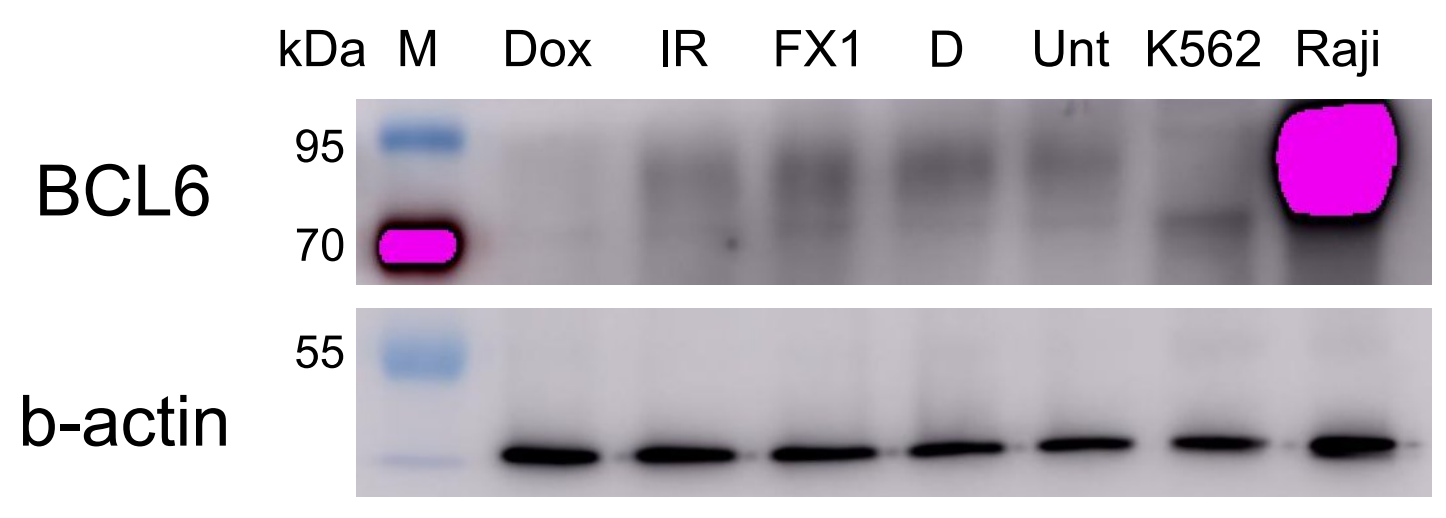

70

a-tubulin

55

Figure 3.4: BCL6 is not up-regulated in response to treatment in U87-MG cells. Western Blot was performed for BCL6, $\beta$-actin and $\alpha$-tubulin. U87-MG cells were treated with either $3 \mu \mathrm{M}$ doxorubicin (Dox), 10 Gy irradiation (IR), $25 \mu \mathrm{M}$ FX1 (FX1), volume equivalent DMSO (D) or left untreated (Unt) and harvested for protein 48 hours after treatment. K562 (BCL6 negative) and Raji (BCL6 positive) cells were harvested at the same time to serve as controls for BCL6 expression. Both $\beta$-actin and $\alpha$-tubulin are shown as loading controls. M indicates size marker. Blot shown is representative of 3 independent experiments. 
Protein expression of BCOR, NCOR, and SMRT was observed in LN18 cells under all treatment conditions except for doxorubicin treatment (Figure 3.5). Cells treated with doxorubicin appeared to have reduced or no expression of all three corepressors. This was unusual as transcript levels for all three co-repressors in doxorubicin treated cells were at a similar level to the other treatments, which indicated that something had prevented the translation of these transcripts.

In Figure 3.6, protein expression of BCOR, NCOR, and SMRT was observed in U87-MG cells. U87-MG cells had a slight reduction in expression of all corepressors with doxorubicin treatment, similar to what was observed with LN18 cells. U87-MG cells seemed to have lower expression of the BCOR and SMRT corepressors across all treatment groups compared to NCOR expression, which reflected the slightly lower transcript observed in Figure $\mathbf{3 . 2}$ for these corepressors. 


\section{kDa M Dox IR FX1 D Unt K562 Raji BCOR $_{130}^{250}-\cdots \cdots$}

NCOR

\section{0}

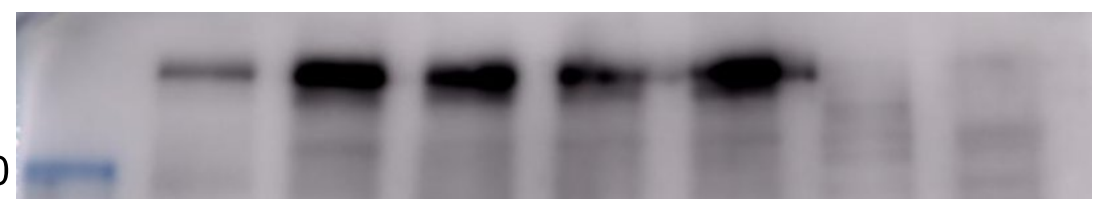

SMRT
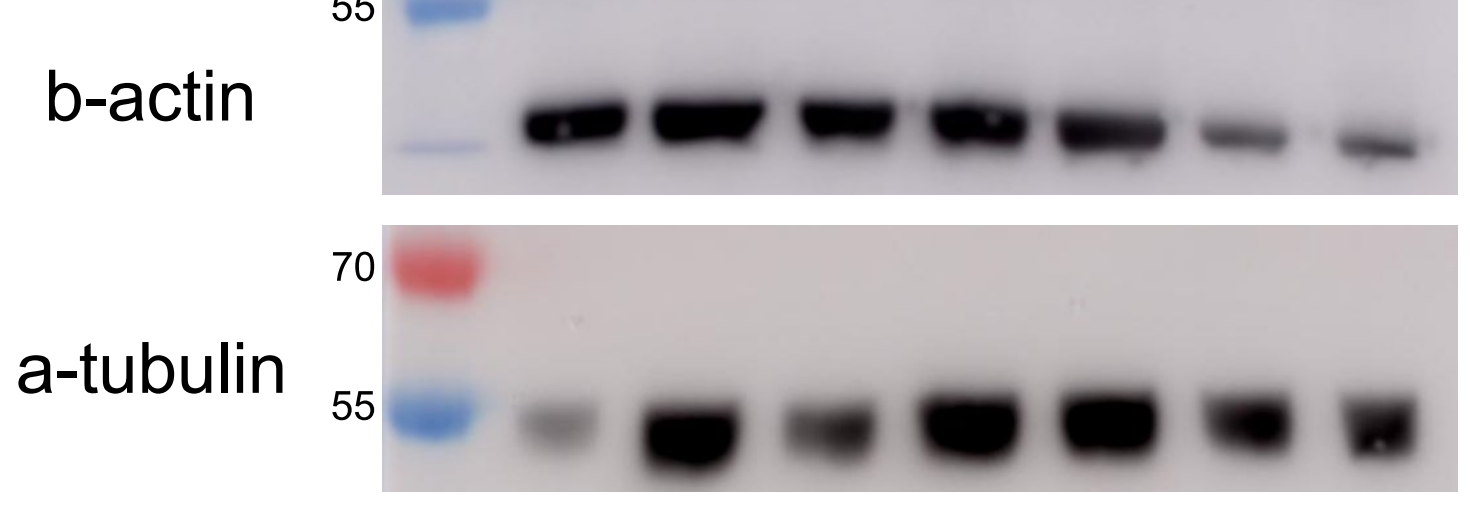

Figure 3.5: The co-repressors BCOR, NCOR, and SMRT are present in LN18 cells. Western Blot was performed for BCOR, NCOR, SMRT, $\beta$-actin and $\alpha$-tubulin. LN18 cells were treated with either $3 \mu \mathrm{M}$ doxorubicin (Dox), 10 Gy irradiation (IR), $25 \mu \mathrm{M}$ FX1 (FX1), volume equivalent DMSO (D) or left untreated (Unt) and harvested for protein 48 hours after treatment. K562 (BCL6 negative) and Raji (BCL6 positive) cells were harvested at the same time to serve as controls for BCL6 expression. Both $\beta$-actin and $\alpha$-tubulin are shown as loading controls. M indicates size marker. Blot shown is representative of 3 independent experiments. 


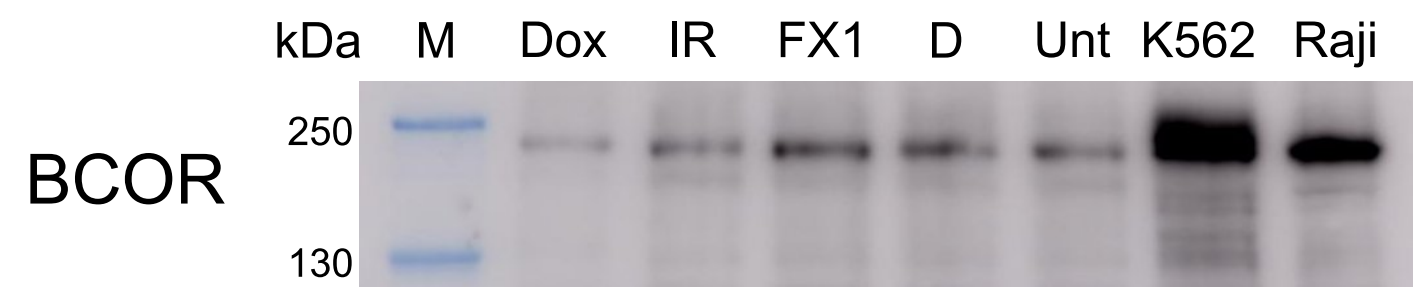

NCOR

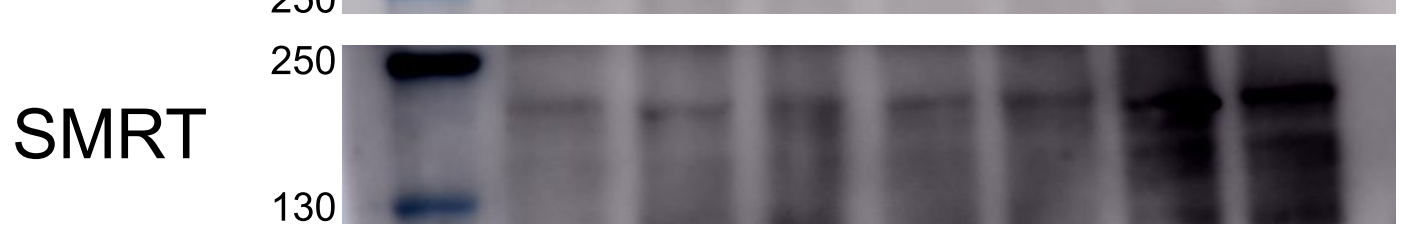

b-actin

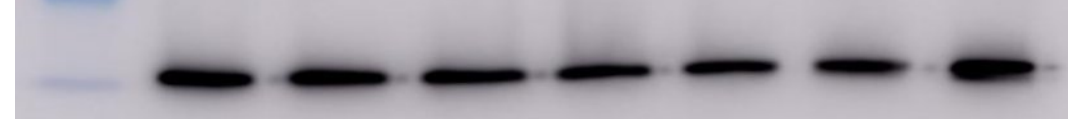

70

a-tubulin

55

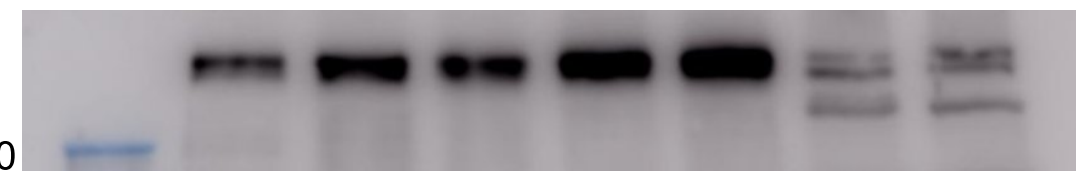

(1)

Figure 3.6: The co-repressors BCOR, NCOR, and SMRT are present in U87MG cells. Western Blot was performed for BCOR, NCOR, SMRT, $\beta$-actin and $\alpha$ tubulin. U87-MG cells were treated with either $3 \mu \mathrm{M}$ doxorubicin (Dox), $10 \mathrm{~Gy}$ irradiation (IR), $25 \mu \mathrm{M}$ FX1 (FX1), volume equivalent DMSO (D) or left untreated (Unt) and harvested 48 hours after treatment. K562 (BCL6 negative) and Raji (BCL6 positive) cells were harvested at the same time to serve as controls for BCL6 expression. Both $\beta$-actin and $\alpha$-tubulin are shown as loading controls. $\mathrm{M}$ indicates size marker. Blot shown is representative of 3 independent experiments. 


\subsubsection{Transcriptional Activity of BCL6 in LN18 cells}

Once presence of BCL6 and its co-repressors was established in LN18 cells, transcriptional activity of BCL6 was assessed by luciferase assay. This required the transfection of GBM cells with a BCL6 linked luciferase plasmid (BCL6 4 -tkLUC) (196). This plasmid has 4 BCL6 consensus binding sites that are upstream of the thymidine kinase (TK) promoter, which initiates luciferase expression. If BCL6 was present and active as a transcriptional repressor it shut down expression of this plasmid, which lead to a reduction in luciferase expression. This reduced expression was observed as less light emitted in the reaction with luciferin as compared to a control where BCL6 was not present. Initially, experiments were conducted with the $\mathrm{BCL6}_{4}$-tkLUC plasmid alongside a GFP control for transfection efficiency, however it was found that doxorubicin and irradiation treatment decreased the expression of GFP, so the reduction in luciferase initially observed may not have been due to BCL6 expression. As a result of this, a control luciferase plasmid was used and the experiments were repeated side-by-side. The control luciferase plasmid used for this assay was the pGL3-Control plasmid (PGL3) (Promega, USA), which has no BCL6 consensus binding sequences, and a Simian Virus 40 (SV-40) promoter for luciferase expression.

In the past, transfection of GBM cells has been proven to be quite difficult, as transfection reagents can have a profound effect on cell health. In Figure 3.7, a comparison between Thermo Fisher's LTX and Plus and Promega's Viafect is shown. In (Figure 3.7A) pictures of GFP, BCL6 ${ }_{4}$-tkLUC, and PGL3 transfected LN18 cells are shown for both reagents. With LTX and Plus there were many floating cells and the cells that were attached did not appear to be healthy. However, with Viafect there were hardly any floating cells and the cells appeared to be healthy. In (Figure 3.7B) transfection efficiency with both reagents was assessed by flow cytometry for GFP positive cells. Cells were first gated to exclude debris and then transfection efficiency was determined by percent positive GFP positive cells. The non-fluorescent cells transfected with Viafect have considerably more debris than the non-transfected cells exposed to LTX and Plus as they have been transfected with a BCL6 dominant negative plasmid which negatively affects the cells. LTX and Plus gave a transfection efficiency of $37.7 \%$, whereas Viafect gave a transfection efficiency of $83.6 \%$, with much less debris seen compared to the non-fluorescent 
control. Given the improved cell health and high transfection efficiency with the Viafect reagent, all transfections with LN18 cells were conducted with Viafect reagent. 
A.

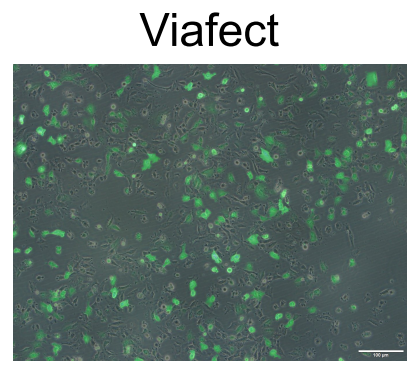

\section{BCL6 4 -tkLUC}
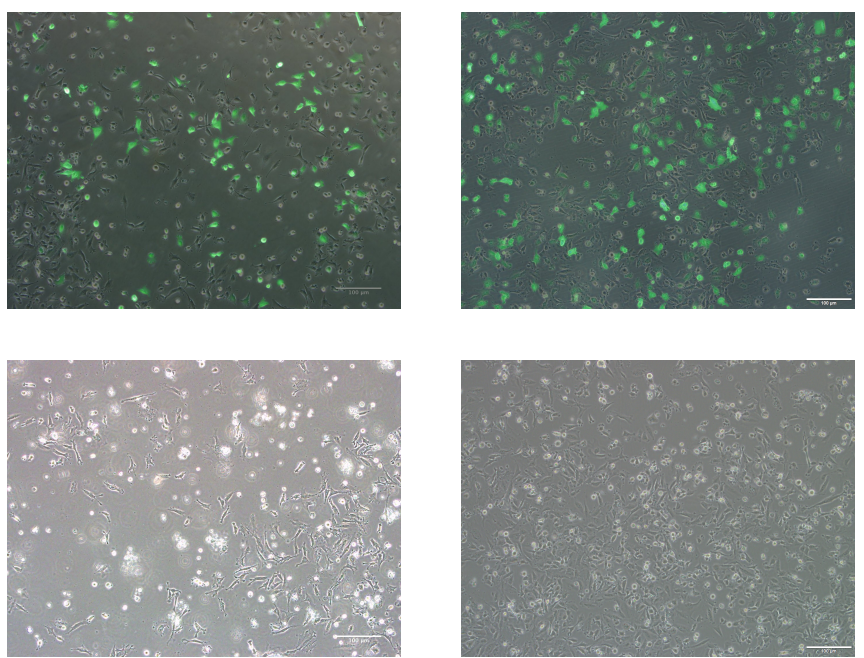

PGL3
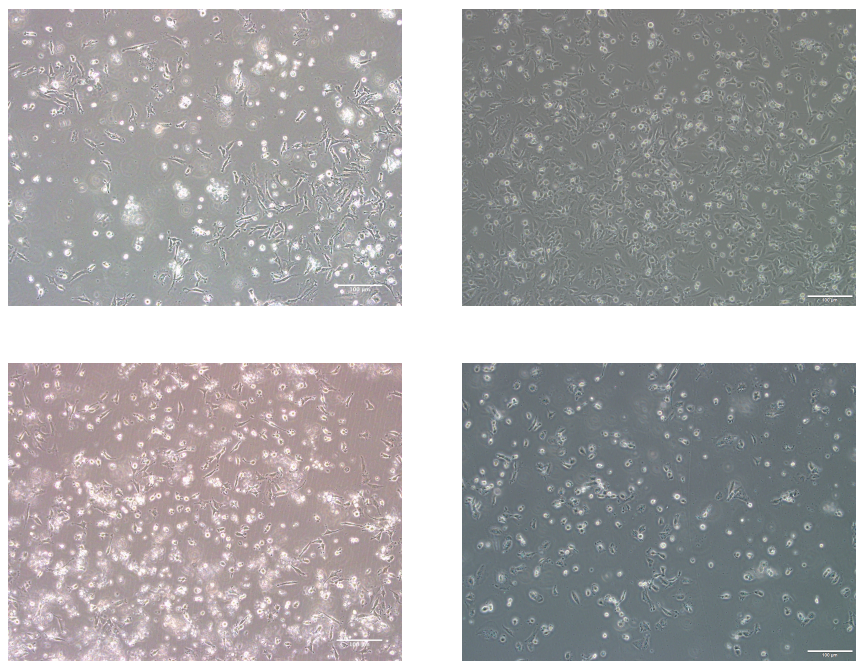

B.
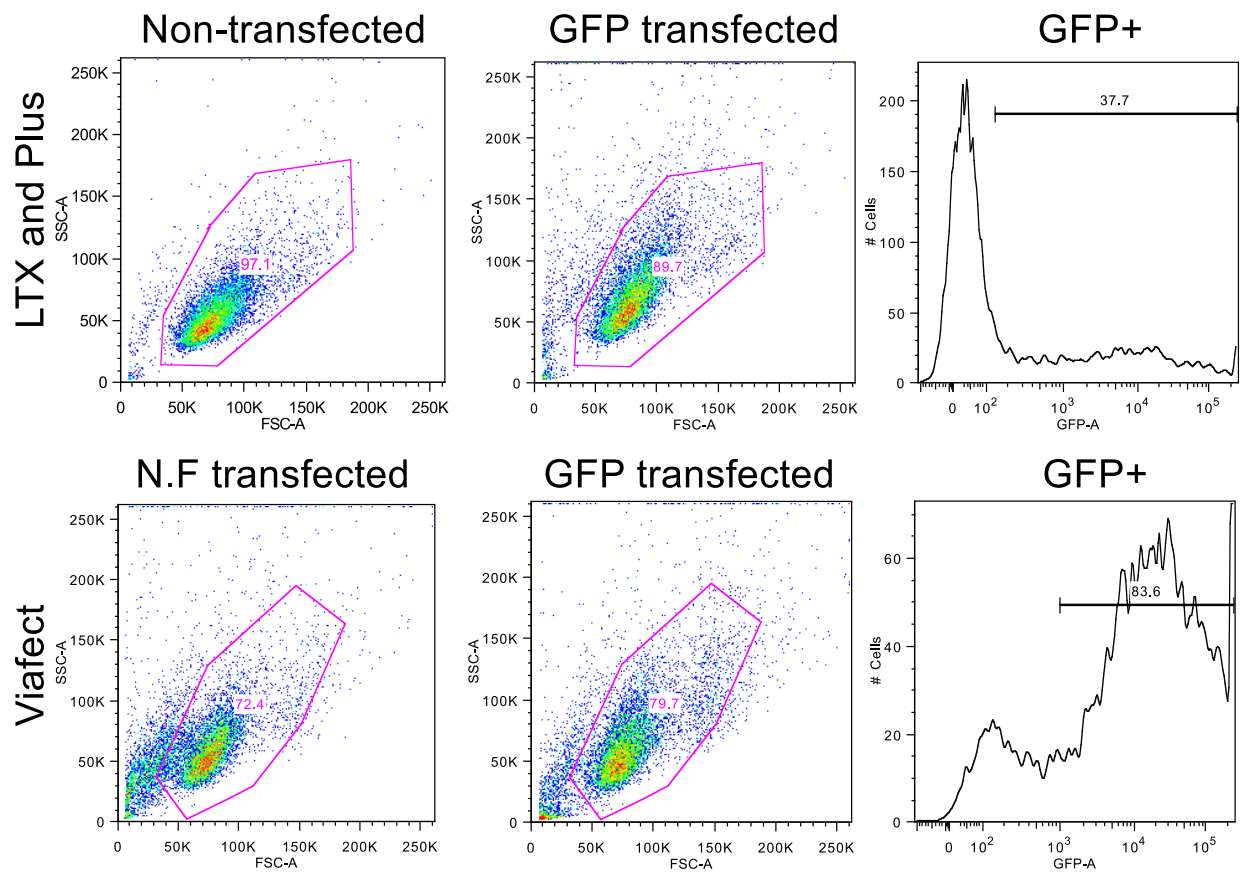

Figure 3.7: Effect of transfection of LN18 with LTX and Plus versus Viafect. (A) LN18 cells transfected with GFP (merge of bright field and fluorescence images), BCL6 ${ }_{4}$-tkLUC or PGL3 plasmids using either LTX and Plus or Viafect. Scale bars represent $100 \mu \mathrm{m}$. (B) LN18 cells transfected with GFP with LTX and Plus or Viafect as indicated were analysed by flow cytometry to determine the transfection efficiency. Scatter plots show whole population of cells recorded, histograms show percent GFP+ cells as compared to a non-transfected or nonfluorescent plasmid (N.F) control. 
To determine if BCL6 was capable of acting as a transcriptional repressor in LN18, cells were co-transfected with a BCL6 overexpression plasmid with a CMV promoter (BCL6wt) and the $\mathrm{BCL6}_{4}$-tkLUC vector (Figure $3.8 \mathrm{~A}$ ).

Expression of BCL6 in co-transfected LN18 cells was able to repress expression of the $\mathrm{BCL6}_{4}$-tkLUC as seen by a significant reduction in luminescence (Figure $\mathbf{3 . 8}$ B).This was shown to be BCL6 specific as co-transfection of BCL6wt with PGL3 did not show the same reduction in light.

To determine if the endogenous BCL6 observed in LN18 (Figure 3.3) was also a transcriptional repressor, cells transfected with the $\mathrm{BCL}_{4}$-tkLUC plasmid were treated with the BCL6 inhibitor FX1 (Figure 3.9). Compared to the non-specific PGL3 plasmid, luciferase activity with the $\mathrm{BCL}_{4}$-tkLUC plasmid was reduced with FX1 treatment, but not to the same extent as seen with overexpressed BCL6. FX1 prevents the binding of co-repressors to BCL6, so it would be expected that treatment with FX1 would inhibit the transcriptional repressor activity of BCL6. As a result, BCL6 would not be able to shut down luciferase expression and an increase in luciferase would be observed with FX1 treatment. Instead, a slight reduction in activity was observed as compared to the untreated control, which indicated that endogenous BCL6 was acting differently to the overexpressed BCL6. 


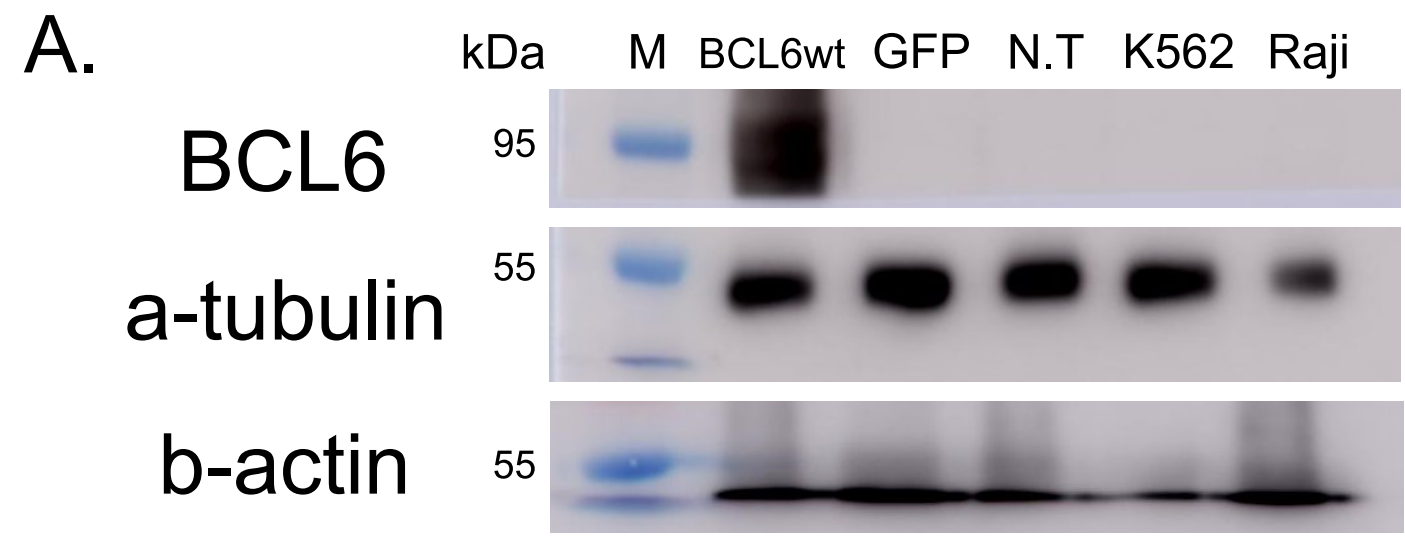

B.

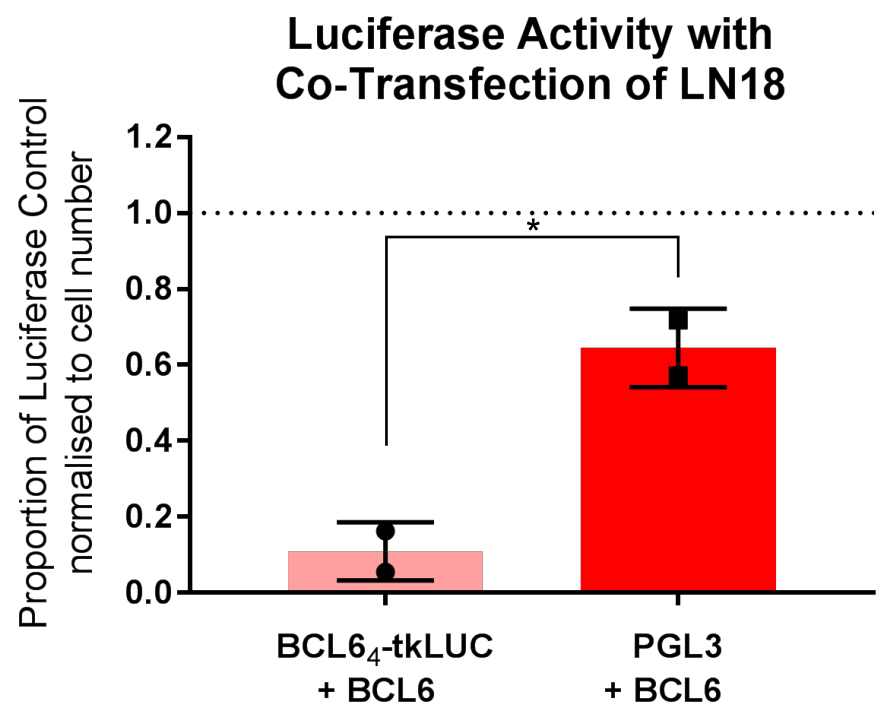

Figure 3.8: Overexpressed BCL6 is a transcriptional repressor in LN18 cells. (A) Western Blot for BCL6 of LN18 transfected with pcDNA-BCL6 (BCL6wt), pcDNA3-GFP (GFP) and non-transfected cells (N.T) alongside K562 (BCL6 positive) and Raji (BCL6 negative) cell lysate. (B) Luciferase assay was performed on LN18 cells co-transfected with either BCL6 $6_{4}$-tkLUC and pcDNA-BCL6 or PGL3 and pcDNA-BCL6 harvested 48 hours after transfection. Luciferase values were normalised to cell number and then compared to single transfected luciferase (either $\mathrm{BCL6}_{4}$-tkLUC or PGL3) control, as indicated by the dotted line on the graph. Bars represent the mean of 2 independent experiments and error bars represent \pm standard deviation. ${ }^{*} \mathrm{p}<0.05$, Student's t-test. 


\section{Luciferase Activity in LN18 cells after $25 \mu \mathrm{M}$ FX1}

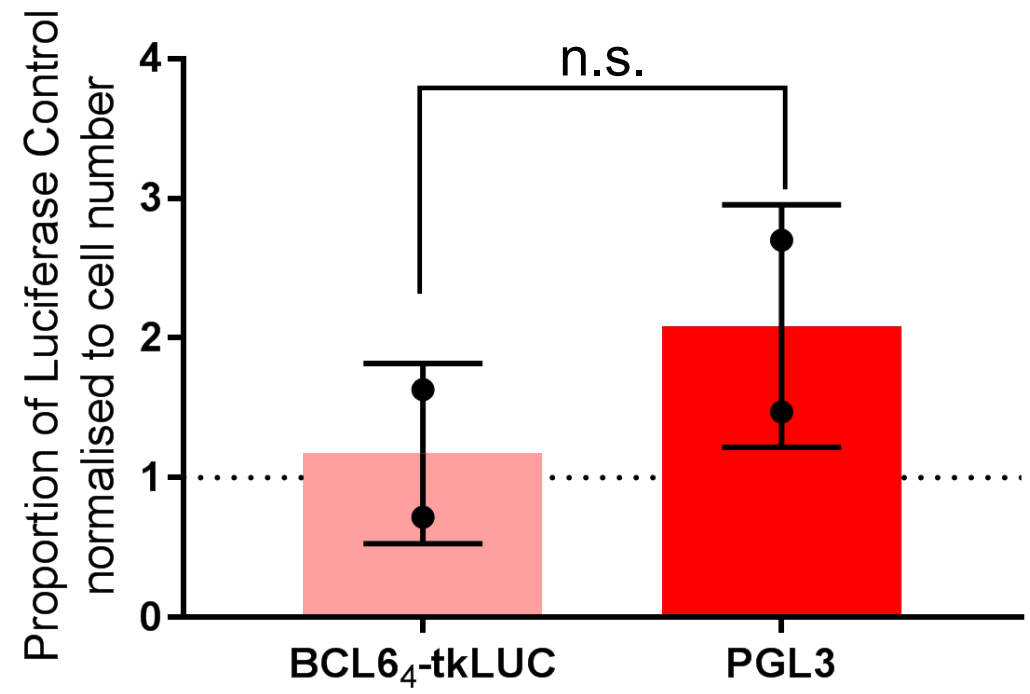

Figure 3.9: Endogenous BCL6 is not transcriptionally active in LN18 cells. Luciferase assay was performed on LN18 cells transfected with either BCL6 $4^{-}$ tkLUC or PGL3 and treated with $25 \mu \mathrm{M}$ FX1 for 48 hours. Luciferase values were normalised to cell number and then compared to equivalent volume DMSO treated and transfected luciferase (either $\mathrm{BCL}_{4}$-tkLUC or PGL3) control, as indicated by the dotted line. Bars represent the mean of 2 independent experiments and error bars represent \pm standard deviation. ns $=$ not significant, Student's t-test. 
As seen in Figure 3.3, BCL6 expression was increased by doxorubicin treatment in LN18 cells. To determine if this therapy induced BCL6 was able to act as a transcriptional repressor, LN18 cells were transfected with BCL6 $_{4}$-tkLUC and treated with doxorubicin (Figure 3.10).

Doses of $50 \mathrm{nM}, 100 \mathrm{nM}$, and $200 \mathrm{nM}$ doxorubicin were used so that the cells were not killed by the combination of doxorubicin and transfection. Doxorubicin treatment of transfected LN18 cells led to a slight but not significant increase in luciferase activity as compared to the non-specific PGL3 control, which may have indicated that BCL6 induced by doxorubicin in LN18 could be a transcriptional activator.

Cells transfected with $\mathrm{BCL6}_{4}$-tkLUC and treated with radiation saw a significant 3fold increase in luciferase activity compared to non-specific PGL3 control plasmid (Figure 3.11). This increase occurred even though no increase in BCL6 expression was seen with radiation in LN18 (Figure 3.1 and Figure 3.3), and provided stronger evidence that BCL6 in LN18 could be a transcriptional activator. 


\section{Luciferase Activity in LN18 cells after Doxorubicin Treatment}

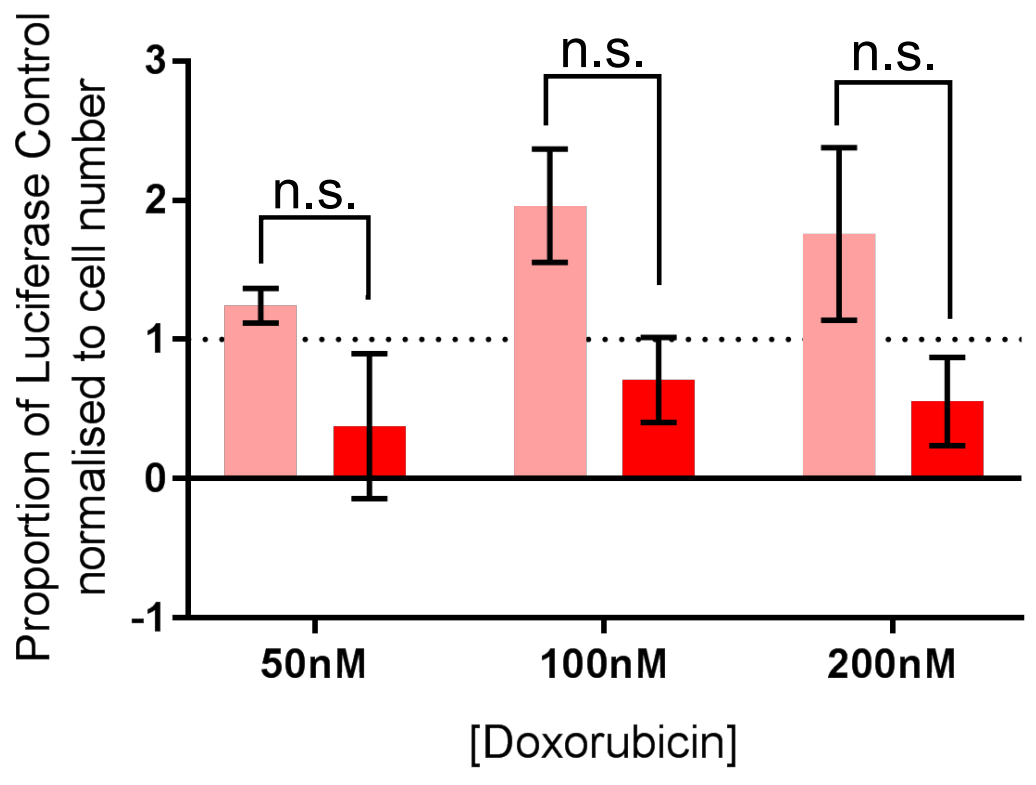

Figure 3.10: Therapy induced BCL6 is not a transcriptional repressor in LN18 cells. Luciferase assay was performed on $\mathrm{LN} 18$ cells transfected with either BCL6 $4^{-}$ tkLUC or PGL3 and treated with $50 \mathrm{nM}, 100 \mathrm{nM}$ or $200 \mathrm{nM}$ doxorubicin for 48 hours. Luciferase values were normalised to cell number and then compared to untreated transfected luciferase (either $\mathrm{BCL}_{4}$-tkLUC or PGL3) control. Bars represent the mean of 2 independent experiments and error bars represent \pm standard deviation. n.s. $=$ not significant, Student's t-test. 


\section{Luciferase Activity in LN18 cells after 10 Gy Radiation}

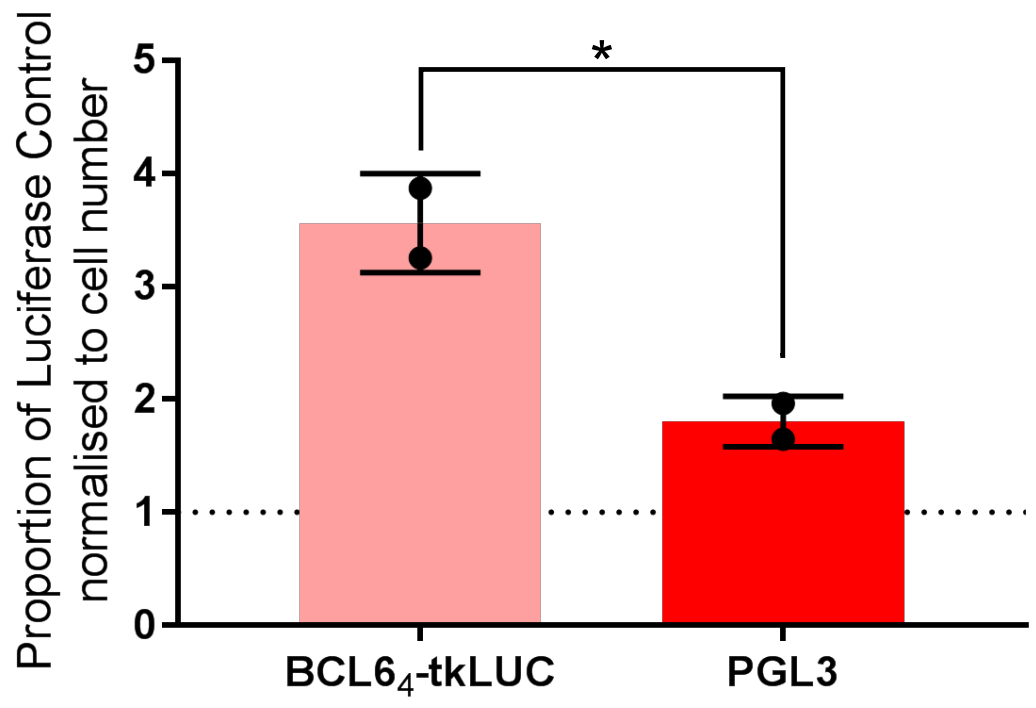

Figure 3.11: Radiation treatment leads to an increase in BCL6 $_{4}$-tkLUC activity. Luciferase assay was performed on LN18 cells transfected with either BCL6 $_{4}$-tkLUC or PGL3 and treated with 10 Gy ionising radiation and harvested 48 hours after treatment. Luciferase values were normalised to cell number and then compared to untreated transfected luciferase (either BCL6 ${ }_{4}$ tkLUC or PGL3) control. Bars represent the mean of 2 independent experiments and error bars represent \pm standard deviation. $* \mathrm{p}<0.05$, Student's t-test. 


\subsubsection{Transcriptional Activity of BCL6 in U87-MG cells}

To determine if BCL6 was able to act as a transcriptional repressor in U87-MG cells, BCL6 transcriptional activity was investigated with the BCL6 $6_{4}$ tkLUC plasmid. As in Figure 3.7, transfection in U87-MG was assessed with both LTX and Plus and Viafect (Figure 3.12). In Figure 3.12A pictures of U87-MG cells transfected with either LTX and Plus or Viafect are shown. Like with LN18 cells, U87-MG cells seemed quite unhealthy with LTX and Plus, with many floating cells. With Viafect the cells were much more adherent, and seemed much healthier. Transfection efficiency was assessed in U87-MG cells transfected with LTX and Plus by flow cytometry for GFP positive cells in Figure 3.12B, which showed a transfection efficiency of $27.5 \%$. U87-MG cells transfected with Viafect were assessed for transfection efficiency by visual inspection of GFP (Figure 3.12A), which showed an increase in GFP positive cells, to $\sim 40 \%$. Overall, Viafect was a far superior transfection reagent to LTX and Plus and was used in all transfections with U87-MG. 
A.

GFP

\section{$\mathrm{BCL6} 6_{4} \mathrm{tkLUC}$}

PGL3
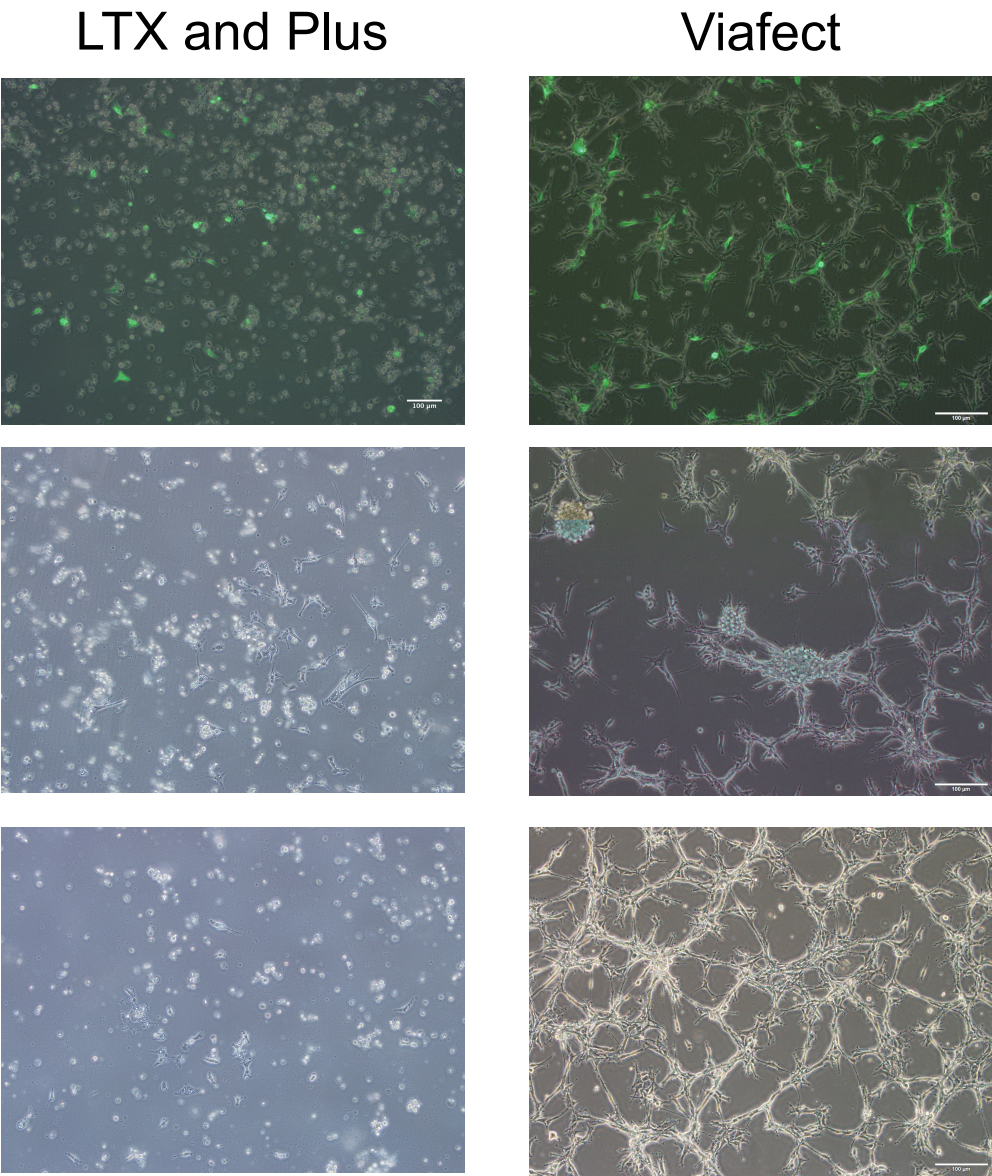

\section{B. Non-Transfected}

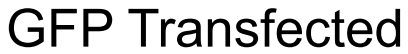

GFP+
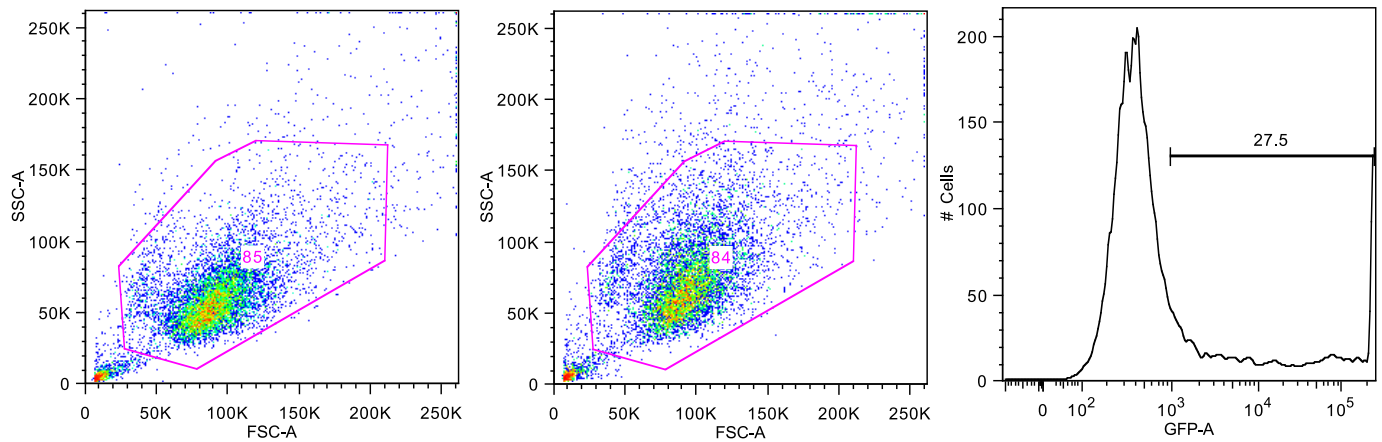

Figure 3.12: Effect of transfection on U87-MG cells with LTX and Plus versus Viafect. (A) U87-MG cells transfected with GFP (merge of bright field and fluorescence images), BCL6 $6_{4}$-tkLUC or PGL3 plasmids using either LTX and Plus or Viafect. Scale bars represent $100 \mu \mathrm{m}$. (B) U87-MG cells transfected with GFP with LTX and Plus were analysed by flow cytometry to determine the transfection efficiency. Scatter plot shows whole population of cells recorded, gated to exclude debris. Histogram shows percent GFP+ cells as compared to the non-transfected control. 
To determine if BCL6 was capable of acting as a transcriptional repressor in U87MG, cells were co-transfected with a BCL6 overexpression plasmid (BCL6wt) and the $\mathrm{BCL6}_{4}$-tkLUC vector. Co-transfected U87-MG cells were not able to repress expression of the $\mathrm{BCL6}_{4}$-tkLUC as shown by no reduction in light (Figure 3.13B) similar to the non-specific PGL3 control plasmid. This would indicate that even when large amounts of BCL6 was present in U87-MG cells (Figure 3.13A) it was unable to affect transcription in a meaningful way, and subsequently was not a useful model for the study of the transcriptional activity of BCL6 in glioblastoma. 


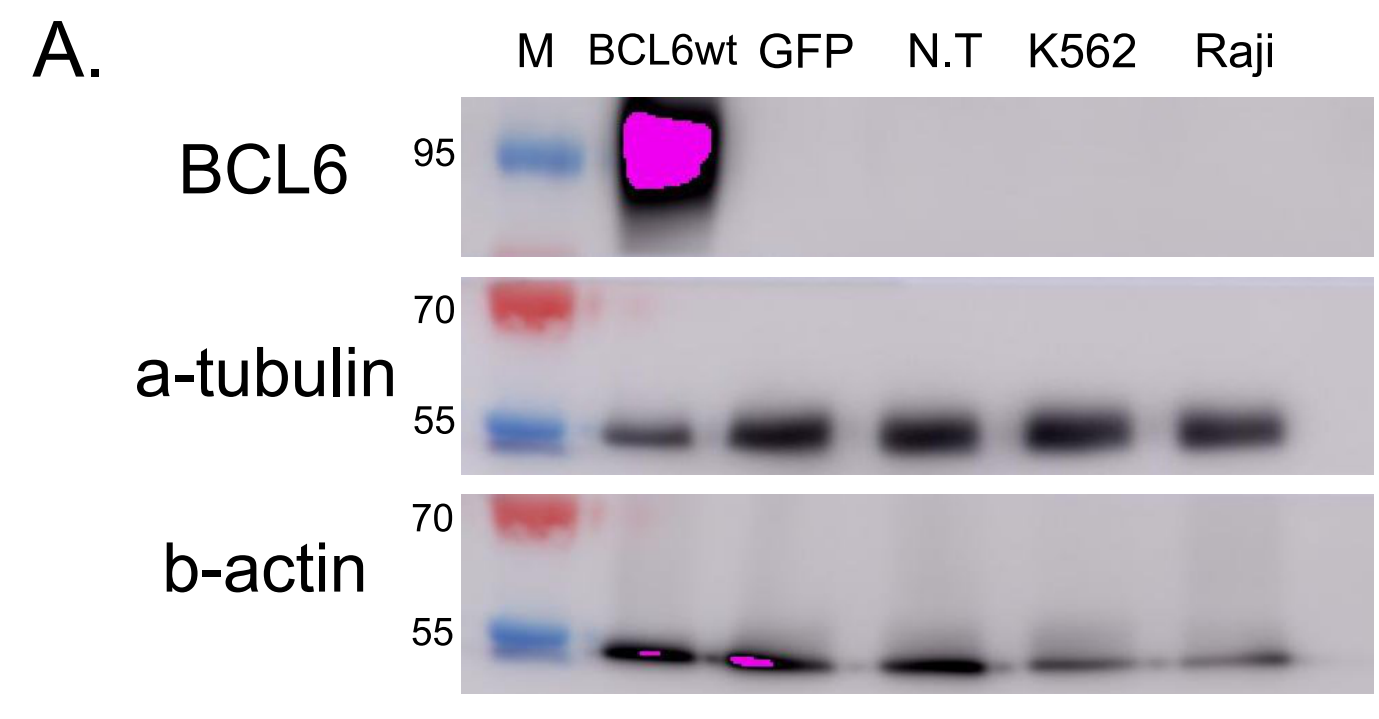

B.

\section{Luciferase Activity with Co-Transfection of U87}

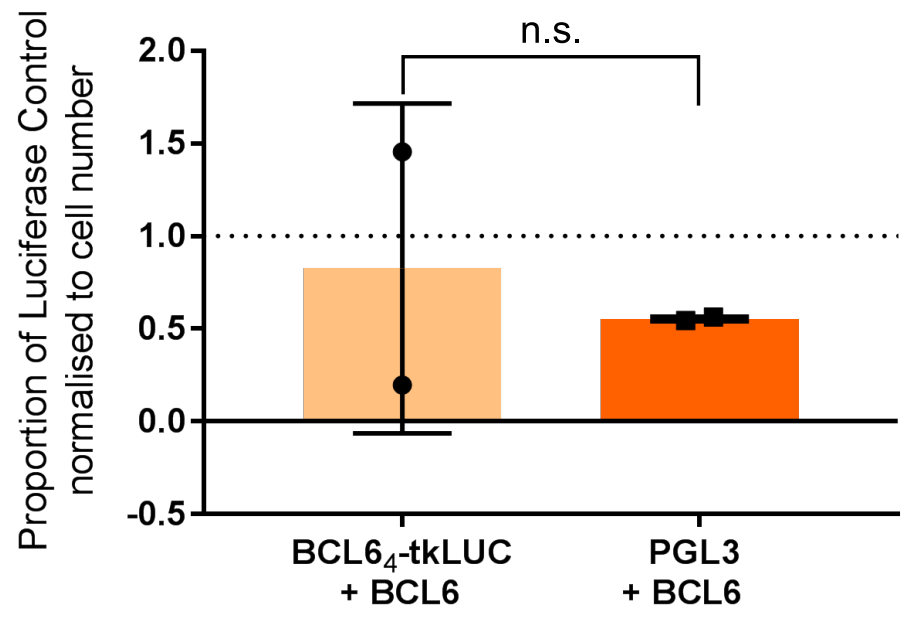

Figure 3.13: Overexpressed BCL6 is not a transcriptional repressor in U87MG cells. (A) Western Blot for BCL6 of U87-MG transfected with pcDNA-BCL6 (BCL6wt), pcDNA3-GFP (GFP) and non-transfected cells (N.T) alongside K562 (BCL6 positive) and Raji (BCL6 negative) cell lysate. (B) Luciferase assay was performed on U87-MG cells co-transfected with either BCL6 $6_{4}$-tkLUC and BCL6 or PGL3 and BCL6 harvested 48 hours after transfection. Luciferase values were normalised to cell number and then compared to single transfected luciferase (either BCL6 $_{4}$-tkLUC or PGL3) control. Bars represent the mean of 2 independent experiments and error bars represent \pm standard deviation. n.s. $=$ not significant, Student's t-test. 


\subsection{Summary}

BCL6 plays an important role in prevention of cell death in response to DNA damage and therapy for glioblastoma relies on this response to DNA damage to induce cell death. Consequently, expression of BCL6 is a good candidate for a mechanism of therapy resistance in glioblastoma. BCL6 is generally described as a transcriptional repressor that operates by the recruitment of co-repressor proteins in order to shut down DNA damage response genes (151). Based on this known mechanism, it would be expected that if BCL6 expression were a mechanism of therapy resistance in glioblastoma it would act as a transcriptional repressor.

The results presented in this chapter have shown that BCL6 levels were increased in LN18 in response to doxorubicin, but not radiation induced DNA damage, which indicated a different response to these two types of therapies. It is unclear whether this increase observed in LN18 is due to an increase in transcription or simply a decrease in $\mathrm{mRNA} /$ protein turnover, as a small increase in transcription of BCL6 was observed with doxorubicin treatment, but this doesn't seem to match the large increase in protein observed. Conversely, BCL6 levels were not increased by doxorubicin treatment in U87-MG cells, but U87-MG maintained endogenous BCL6 expression in all other treatment groups. Co-repressors BCOR, NCOR, and SMRT were all expressed in both LN18 and U87-MG under all treatment conditions, with the exception of doxorubicin treated LN18 cells, which had reduced expression of the co-repressors. It seems unlikely that the reduced expression of the co-repressors in doxorubicin treated LN18 cells is related to the increased BCL6 expression in LN18 cells with doxorubicin treatment, but this may partially explain why no transcriptional repressor activity was seen with doxorubcin treatment in LN18 cells; they don't have the machinery.

Overexpressed BCL6 in LN18 cells operated effectively as a transcriptional repressor, but this was not the case in U87-MG cells, despite expression of corepressors being similar in both cell lines. These differences reflect the heterogeneity of GBM tumours. BCL6 expression has been observed in a number of different GBM cell lines (187) (See Appendix: Fabre, Jones, et al., in revision), but whether this translates into transcriptionally active BCL6 has yet to be determined for many of 
these cell lines. BCL6 expression has been shown to vary widely in primary tumours (See Appendix: Fabre, Jones, et al., in revision), so it is likely that BCL6 activity will also vary widely.

When BCL6 was overexpressed in LN18 cells it was able to act as a transcriptional repressor, however this was not the case with BCL6 in doxorubicin or radiation treated cells. In fact a significant increase in transcriptional activity of the reporter was seen with radiation, and there appeared to be an increase in transcriptional activity seen with doxorubicin treatment, though this was not significant. These results indicated that endogenous BCL6 has the potential to act as a transcriptional activator in treated LN18 cells, even when no increase in transcript or protein was seen with treatment. This result is contrary to the hypothesis that BCL6 acts as a transcriptional repressor in GBM, but it is important to note that this is only a single result on one cell line with one treatment, and it is not yet clear whether an increase in transcriptional activity would be seen with this assay in other cells or with other treatments.

As discussed earlier, BCL6 was not present with doxorubicin treatment in U87-MG cells, but was present at a basal level with other treatments and was not transcriptionally active when overexpressed in U87-MG cells. These results indicated that U87-MG is not a good model for the study of the BCL6 mechanism in glioblastoma. Accordingly, the remaining chapters of this thesis will focus on the cell line LN18. 


\section{DNA Binding of BCL6 in Glioblastoma}

\subsection{Introduction}

BCL6 binds to its consensus binding sequence (TTCCTAGAA) directly through its 6 zinc fingers (141), and acts as a transcriptional repressor by the recruitment of corepressors BCOR/NCOR/SMRT to its BTB domain while bound to DNA (164). The co-repressors then recruit HDAC3, which de-acetylates the histone and shuts down gene expression. This mechanism of action has been established in primary B-cells (164), T-follicular helper cells (203), and lymphoma (204). In the context of GBM, it is not known whether BCL6 is able to bind its consensus binding sequence and recruit co-repressors.

BCL6 has a number of target genes that are well described in lymphoma, which include CCND2 (150), ATR (146), TP53 (145), and CHEK1 (147). BCL6 is also autoregulatory, and prevents expression of itself by binding to the promoter of BCL6 Exon 1 (204). When ChIP seq for BCL6 was performed on breast cancer cells and compared to lymphoma, it was found that breast cancer had vastly different target genes for BCL6, with 1079 unique targets identified (180). More recently, studies on T-follicular helper cells have shown that BCL6 has different target genes in these cells, as compared to lymphoma (205). Subsequently it is likely that BCL6 will have different target genes in GBM as compared to lymphoma.

In Chapter 3 it was established that in most conditions co-repressors BCOR, NCOR, and SMRT are present in GBM cells and when BCL6 is overexpressed, it is able to act as a transcriptional repressor. BCL6 was shown to be present in both untreated and treated GBM cells (with doxorubicin and radiation), and it was also was shown that treatment of LN18 with doxorubicin and radiation lead to an increase in reporter activity, which suggested that it may be a transcriptional activator. This result conflicted with BCL6's traditional role of transcriptional repression. To determine if BCL6 is a DNA binding protein in GBM, the DNA binding and target genes of BCL6 were investigated in LN18. 


\subsection{Aims}

The aims of this chapter are to investigate the ability of BCL6 to bind DNA and interact with co-repressors while bound, as well as to see if BCL6 is bound to wellknown BCL6 target genes.

\subsection{Results}

\subsubsection{BCL6 interaction with DNA binding motif}

In order to act as a transcriptional repressor, BCL6 needs to bind DNA and its corepressors BCOR, NCOR, and SMRT. In chapter 3 it was established that corepressors are present in LN18 cells, except when treated with doxorubicin. It was also shown that when BCL6 was overexpressed it was able to act as a transcriptional repressor, but endogenous BCL6 was not. The electrophoretic mobility shift assay (EMSA) technique was used to investigate whether overexpressed/endogenous BCL6 is able to bind its consensus binding sequence and recruit co-repressors to this complex.

As the EMSA technique had not been established in our lab, a control system was set up first with the Lightshift Chemiluminescent EMSA kit (Thermo Fisher, NZ). This kit employed the Epstein-Barr virus nuclear antigen (EBNA) and had included components for producing a shift and competition assay to show that it was specific. In Figure 4.1 this is shown, in lane 1 there was no EBNA protein, and no shift of the probe was seen. In lane 2 there was EBNA protein and a clear shifted band was seen. In lane 3, additional probe that was not labelled with biotin was added in excess to the mix and this competition eliminated the shifted band, as the EBNA protein was bound to the unlabelled probe. This shows that the kit and protocol used was working correctly and that EMSAs with the protein of interest could be performed. 
Lane

EBNA protein

Unlabelled competitor DNA

Biotin labelled probe

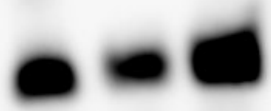

90

$\begin{array}{lll}1 & 2 & 3 \\ - & + & + \\ - & - & + \\ + & + & +\end{array}$

Figure 4.1: Establishment of Electrophoretic Mobility Shift Assay (EMSA) with control from kit. Electrophoretic Mobility Shift Assay with EBNA control system provided in Lightshift Chemiluminescent EMSA kit. 
In chapter 3, BCL6 was shown to be a transcriptional repressor in LN18 cells when overexpressed (Figure 3.8). To show that this repression was caused by the recruitment of co-repressors to BCL6, EMSA on BCL6wt transfected cells was performed. Cells were harvested 24 hours after transfection and a nuclear preparation was made of the cells before incubation with a biotin labelled probe containing the BCL6 consensus binding sequence (206). EMSA was performed in the same manner as Figure 4.1, and control reactions were run alongside every EMSA reaction.

In Figure 4.2 EMSA of BCL6wt transfected LN18 cells is shown. In lane 1, no nuclear extract was added to the probe, and no complexes were seen. In lane 2 nuclear extract was added and 3 distinct complexes were seen $(\mathbf{a}, \mathbf{b}$ and $\mathbf{c})$. In lane 3 unlabelled probe was added in excess to the reaction and successfully competed out the complexes seen in lane 2, which showed that these bands were bound to the sequence specifically. In lane 4, BCL6 antibody was added and complexes a and c were disrupted, which indicated that BCL6 was likely present in these complexes. In lane 5 BCOR antibody was added and complexes $\mathbf{a}$ and $\mathbf{b}$ were disrupted, which indicated that BCOR was likely present in these complexes. In lane 6 NCOR antibody was added, and complex $\mathbf{a}$ was shifted and complex $\mathbf{b}$ was disrupted, which indicated that NCOR was likely present in these complexes. In lane 7 SMRT antibody was added and complexes $\mathbf{b}$ and $\mathbf{c}$ were disrupted, which indicated that SMRT was likely present in these complexes. In lanes 8 and 9 GFP transfected nuclear preparation was added to complex with the BCL6 consensus sequence and no complexes were seen. Overall, complex a appeared to contain BCL6, BCOR and NCOR, complex $\mathbf{b}$ appeared to contain BCOR, NCOR, and SMRT and complex $\mathbf{c}$ appeared to contain BCL6 and SMRT. This showed that BCL6 was able to recruit co-repressors when overexpressed, and this was likely the mechanism of the transcriptional repression seen in Figure 3.8.

To investigate the ability of endogenous BCL6 in GBM to bind the consensus sequence, EMSA was performed on nuclear extracts of untreated and doxorubicin treated LN18. Cells were treated with $0.05 \mu \mathrm{M}, 0.1 \mu \mathrm{M}, 0.2 \mu \mathrm{M}$, and $3 \mu \mathrm{M}$ 
doxorubicin or left untreated, and EMSA was performed 48 hours after treatment, as BCL6 protein level was increased at this time-point post doxorubicin treatment.

In Figure 4.3 EMSA of doxorubicin treated LN18 cells is shown. In lane 1, no nuclear extract was added with the probe, and no complexes were seen. In lanes 26 , nuclear extract of cells untreated, or treated with $0.05 \mu \mathrm{M}, 0.1 \mu \mathrm{M}, 0.2 \mu \mathrm{M}$ or 3 $\mu \mathrm{M}$ doxorubicin, respectively were incubated with the BCL6 specific probe and no complexes were seen. This result indicated that both endogenous and doxorubicininduced BCL6 in LN18 cells was unable to bind its consensus binding sequence. 

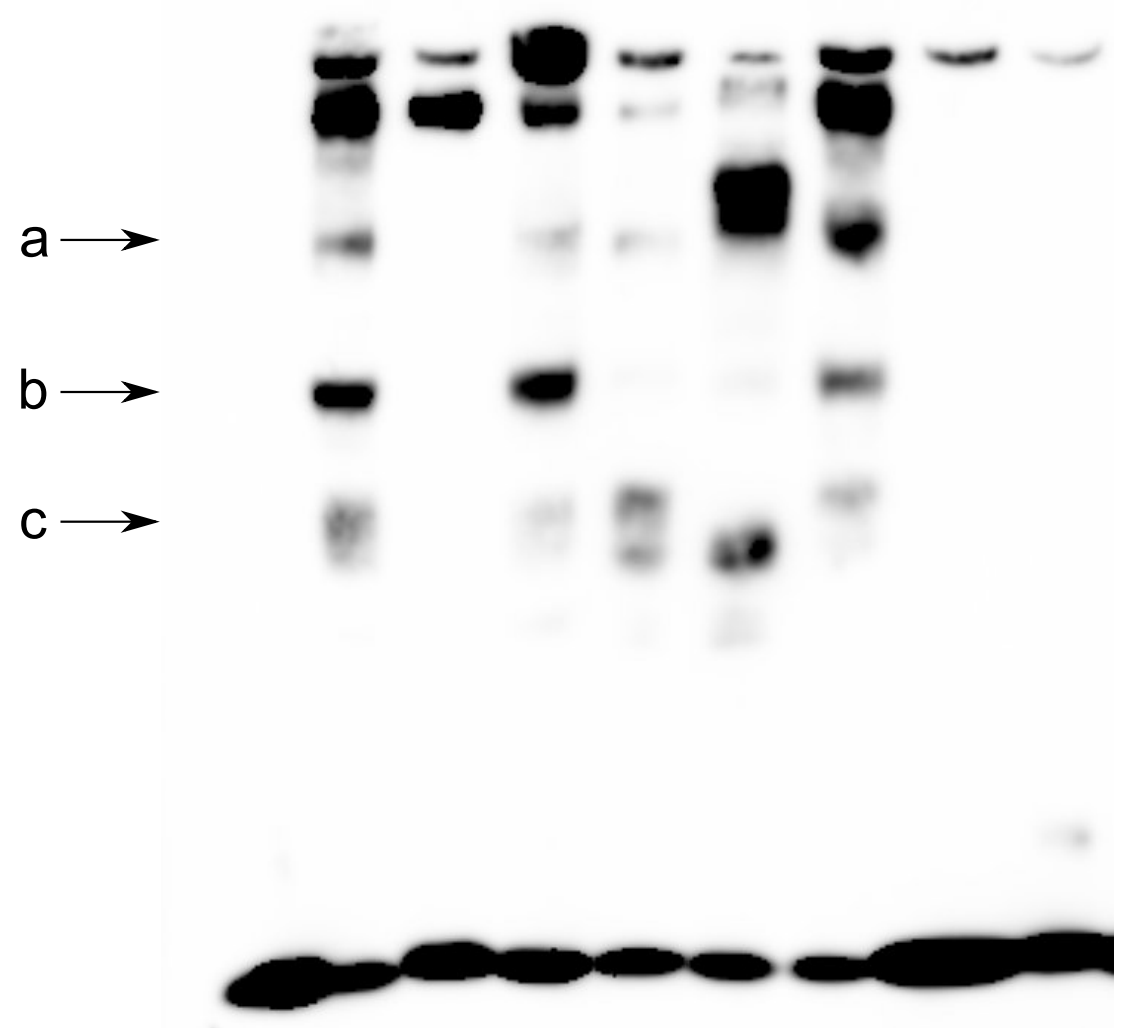

Lane

pcDNA-BCL6

pcDNA3-GFP

Unlabelled competitor DNA

Antibody

Biotin labelled probe

$\begin{array}{ccccccccc}1 & 2 & 3 & 4 & 5 & 6 & 7 & 8 & 9 \\ - & + & + & + & + & + & + & - & - \\ - & - & - & - & - & - & - & + & + \\ - & - & + & - & - & - & - & - & + \\ - & - & - & \text { B1 } & \text { B2 } & \text { N } & \text { S } & - & - \\ + & + & + & + & + & + & + & + & +\end{array}$

Figure 4.2: Overexpressed BCL6 forms distinct complexes with corepressors. Electrophoretic mobility shift assay (EMSA) of LN18 nuclear lysate transfected with pcDNA-BCL6 (lanes 2-7) or pcDNA3-GFP (lanes 8 \& 9). Complexes were formed with nuclear lysate and biotin labelled probe alone (lanes 2 and 8), with unlabelled competitor DNA (lanes 3 and 9), with BCL6 antibody (B1) (lane 4), with BCOR antibody (B2) (lane 5), with NCOR antibody (N) (lane 6), and with SMRT antibody (S) (lane 7). (a) indicates complex formed with BCL6, BCOR, and NCOR. (b) indicates complex formed with BCOR and NCOR. (c) indicates complex formed with BCL6, BCOR and SMRT. Blot shown is representative of 2 independent experiments. 
Lane

Untreated

[Doxorubicin] $(\mu \mathrm{M})$

Biotin labeled probe

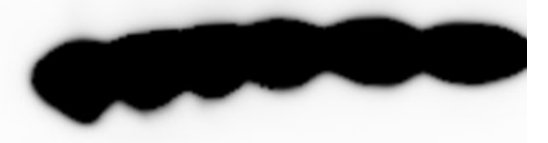

Figure 4.3: Natively expressed BCL6 does not form complexes with BCL6 binding consensus sequence. EMSA of LN18 nuclear lysate treated with 0.05 , $0.1,0.2$ and $3 \mu \mathrm{M}$ doxorubicin (lanes 2-6). Blot shown is representative of 2 independent experiments. 


\subsubsection{Establishment of chromatin immunoprecipitation in LN18}

Chromatin immunoprecipitation (ChIP) is used to investigate the binding of transcription factors or the occurrence of histone alterations along the genome. It involves cross-linking of protein to the DNA followed by immunoprecipitation for the protein of interest and then isolation of the associated DNA for further analysis. ChIP for BCL6 has been well established in lymphoma and BCL6 target genes have been validated using this technique. ChIP had not been done in our lab previously, so every aspect of this technique required optimisation, starting with sonication. Sonication is used to break up the DNA into small fragments after fixing and performing a nuclear preparation on the cells. The fragments need to be around 300 bp in length for optimal resolution, as this is equivalent to 1-2 nucleosomes and is an ideal length for accurate sequencing. Many machines exist to perform sonication, which vary in efficiency, so sonication needs to be optimised for every machine used.

In Figure 4.4 sonication optimisation for LN18 cells is shown. Sonicated DNA is fragmented and able to move freely in an agarose gel, as compared to unsonicated DNA, which cannot move much through an agarose gel. In Figure 4.4A unsonicated DNA is shown (U.S) which had not migrated through the agarose gel. In Figure 4.4B an optimisation of probe sonication with 10, 15 and 20 cycles of 20 s on/ 20 s off at $50 \%$ amplitude is shown. Sonication efficiency increased with every increase in cycles, with the majority band around 300 bp for 20 cycles. In Figure 4.4C and $\mathbf{D}$, representative samples of sonication on untreated, doxorubicin treated and radiation treated LN18 cells are shown (30 cycles of 20s on/ 20s off). Sonication efficiency was not affected by treatment of the cells, as seen by the majority band consistently around 300 bp. Sonication efficiency was checked in every ChIP experiment before proceeding with immunoprecipitation. 

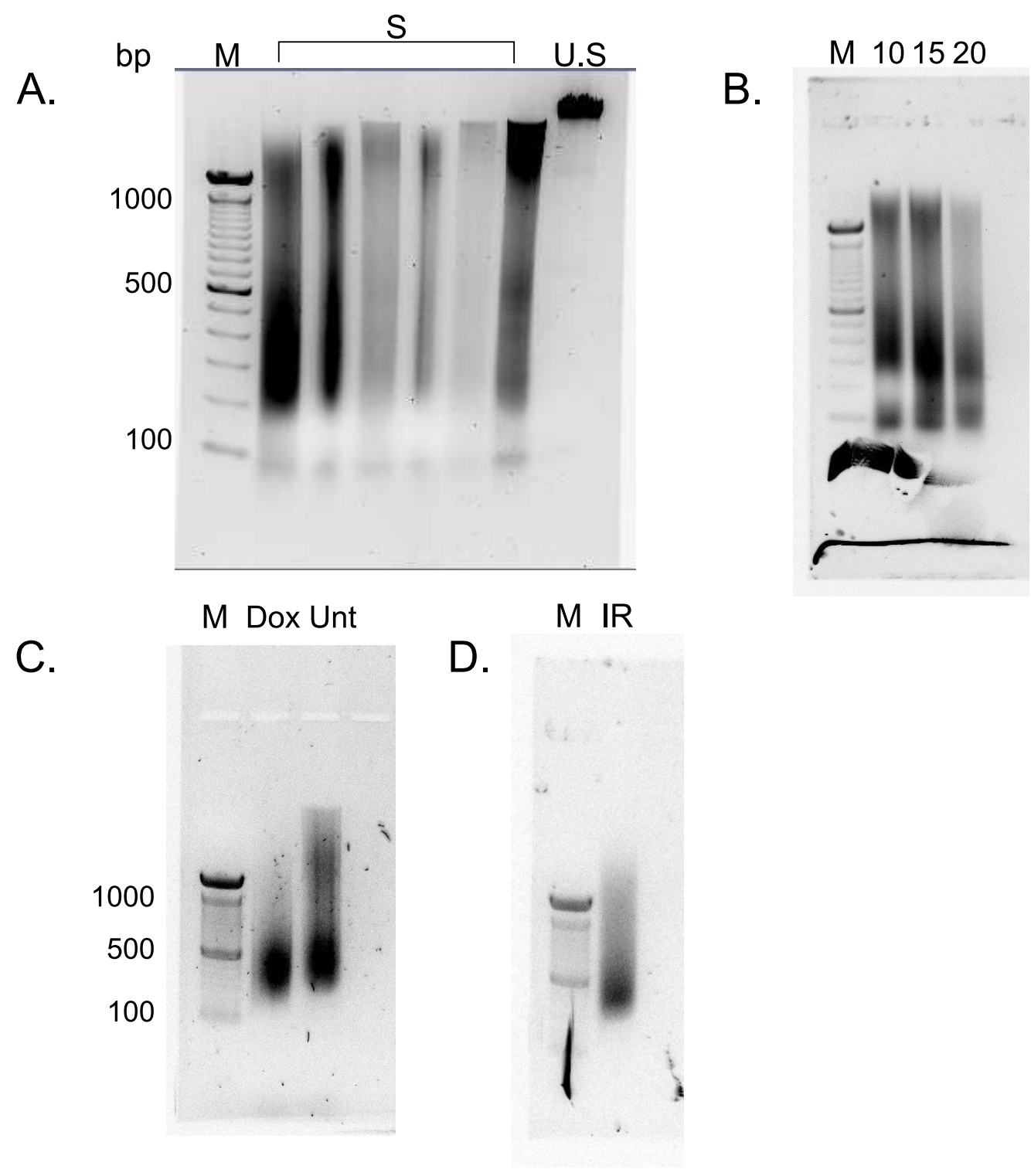

Figure 4.4: Optimisation of sonication protocol for Chromatin Immunoprecipitation (ChIP). LN18 samples were fixed, quenched and had undergone nuclear preparation before sonication. (A) $2 \%$ agarose gel with samples treated with waterbath sonication. $\mathrm{S}$ indicates various sonicated samples, U.S indicates unsonicated DNA sample. (B) $2 \%$ agarose gel of samples treated with 10, 15 , and 20 cycles of $20 \mathrm{~s}$ on $/ 20$ s off sonication at $50 \%$ amplitude with SONOPULS probe sonicator. (C) 1\% agarose gel showing sonication efficiency of representative samples from $3 \mu \mathrm{M}$ doxorubicin (Dox) and untreated (Unt) samples used for ChIP, sonicated for 20 minutes (20s on $/ 20$ s off, $50 \%$ amplitude). (D) $1 \%$ agarose gel showing sonication efficiency of representative sample from a 10 Gy irradiation (IR) sample used for ChIP, sonicated in the same manner as (C). 


\subsection{3 qChIP on Doxorubicin treated LN18 cells}

ChIP experiments were completed at two different sites, with initial ChIP experiments completed in the Melnick lab, under the supervision of Dr. Katerina Hatzi (164,205). The preliminary qChIP (quantitative ChIP) experiments completed in the Melnick lab were used to establish the control genes and investigate potential target genes for BCL6 in LN18. LN18 cells were plated, treated after 24 h with 3 $\mu \mathrm{M}$ doxorubicin, and harvested $48 \mathrm{~h}$ after treatment. Four experiments were completed, with multiple samples taken from each experiment for testing. Different genes were tested in each independent experiment, as detailed in the figure legend. qPCR was completed for target genes on BCL6 and rabbit IgG ChIP samples, $\Delta \mathrm{Ct}$ was calculated against the $\operatorname{IgG}$ sample and fold change was calculated using the $-2^{\Delta \mathrm{Ct}}$ method (207).

In Figure 4.5, the result of qPCR performed on LN18 ChIP samples generated in the Melnick lab is shown. In Figure 4.5A fold change values for positive control BCL6 exon 1 and negative control BCL6 intron 9 are shown. BCL6 appeared to be bound at BCL6 exon 1 with some variation, and not bound at BCL6 intron 9 which validated these as good positive and negative controls for BCL6 ChIP in LN18. In Figure 4.5B fold change values for potential target genes TARS, CCND2, ATR, TP53, PTEN, and CHK1 are shown. BCL6 appeared to be bound at TARS, CCND2, and ATR, though this varied between experiments. BCL6 did not appear to be bound at TP53, PTEN, and CHK1. From these experiments, BCL6 exon 1, BCL6 intron 9, TARS, CCND2 and ATR were chosen to use for experiments conducted back in the McConnell lab at Victoria University of Wellington. 
A.

\section{BCL6 ChIP $3 \mu \mathrm{M}$ Doxorubicin 48 hours}

(Melnick Lab)

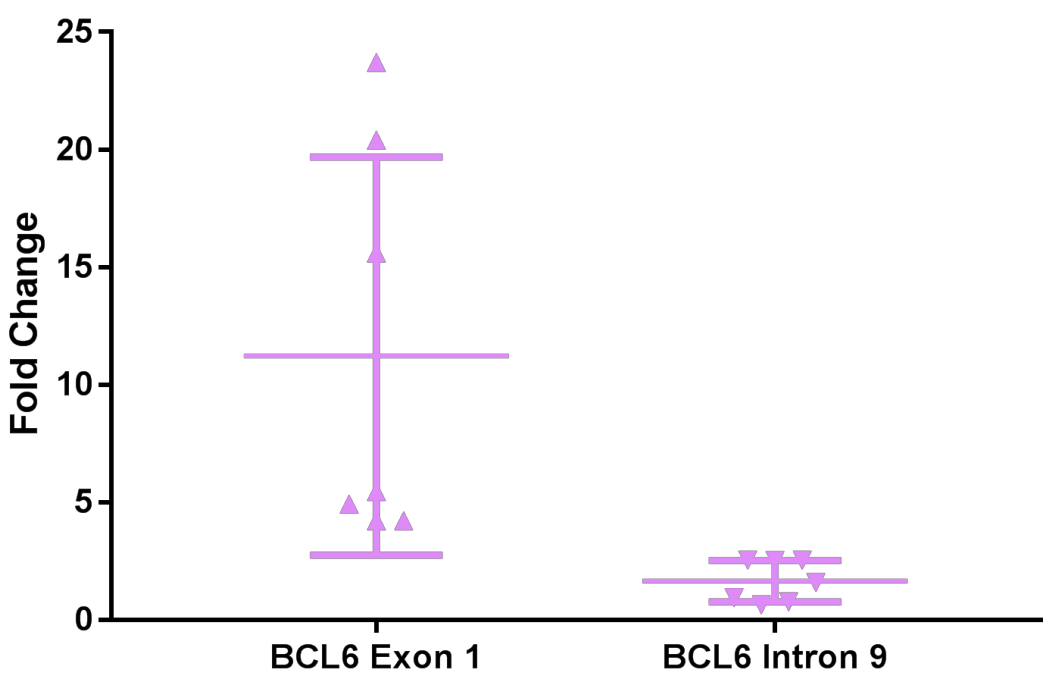

B.

\section{BCL6 ChIP $3 \mu$ M Doxorubicin 48 hours}

(Melnick Lab)

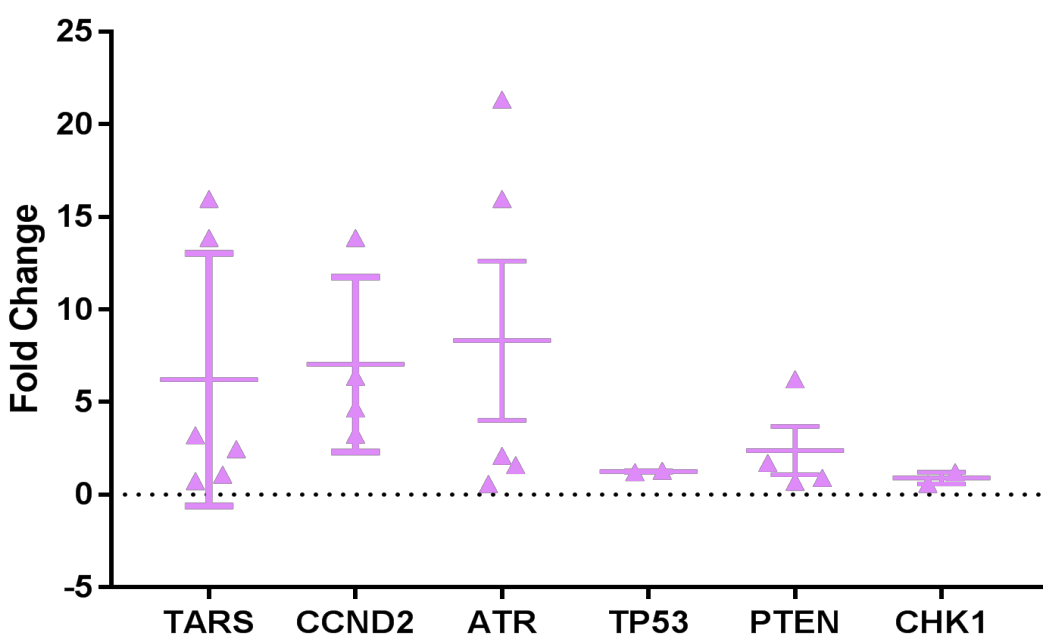

Figure 4.5: Establishment of BCL6 ChIP assay in Melnick Lab at Weill Cornell Medical College, New York City. BCL6 ChIP of LN18 cells treated with $3 \mu \mathrm{M}$ doxorubicin and harvested 48 hours after treatment. (A) qPCR for positive control (BCL6 Exon 1) and negative control (BCL6 intron 9). 3 independent experiments were completed for each. (B) qPCR for known BCL6 target genes TARS, CCND2, ATR, TP53, PTEN, and CHK1. For CCND2 3 independent experiments were completed. For TARS and $A T R 2$ independent experiments were completed. For TP53, PTEN, and CHK1 1 independent experiment was completed. Fold change values calculated using $\Delta \mathrm{Ct}$ method with rabbit IgG as control. Long horizontal lines represent mean \pm standard deviation. 
Once back in New Zealand, the experimental procedure learned in the Melnick lab was adapted to our laboratory. Some changes were made to the protocol- the sonication was with a different machine, but had already been optimised as demonstrated in Figure 4.4, an RNA polymerase II ChIP was performed alongside BCL6 ChIP as a positive control, and a $\beta$-actin antibody was used as the negative control ChIP. LN18 cells were either treated with doxorubicin and harvested 8 hours or 48 hours after treatment, or left untreated and harvested 72 hours after plating.

In Figure 4.6 BCL6 ChIP and RNA polymerase II ChIP qPCR for BCL6 Exon 1 and BCL6 intron 9 are shown. In Figure 4.6A BCL6 ChIP showed binding of BCL6 to BCL6 Exon 1 to be similar across untreated, $8 \mathrm{~h}$ and $48 \mathrm{~h}$ doxorubicin samples $(3.71 \pm 2.79,2.17 \pm 1.68$ and $1.57 \pm 0.728$ fold respectively) though this was at a much lower fold change than that seen in the Melnick lab experiments. It appeared that no binding was seen at BCL6 Intron 9 for all untreated cells and both doxorubicin time-points, which was consistent with results seen in the Melnick lab, and indicated this was a good negative control. In Figure 4.6B there is more binding of RNA polymerase II at BCL6 Exon 1 in untreated cells compared to $8 \mathrm{~h}$ and $48 \mathrm{~h}$ doxorubicin treated cells $(127 \pm 66.4,34.3 \pm 12.3$, and $43.6 \pm 26.3$ fold respectively). No binding was seen at BCL6 Intron 9 with RNA polymerase II ChIP in all samples, which indicated that it was a good negative control. 
A.

BCL6 ChIP $3 \mu$ M Doxorubicin

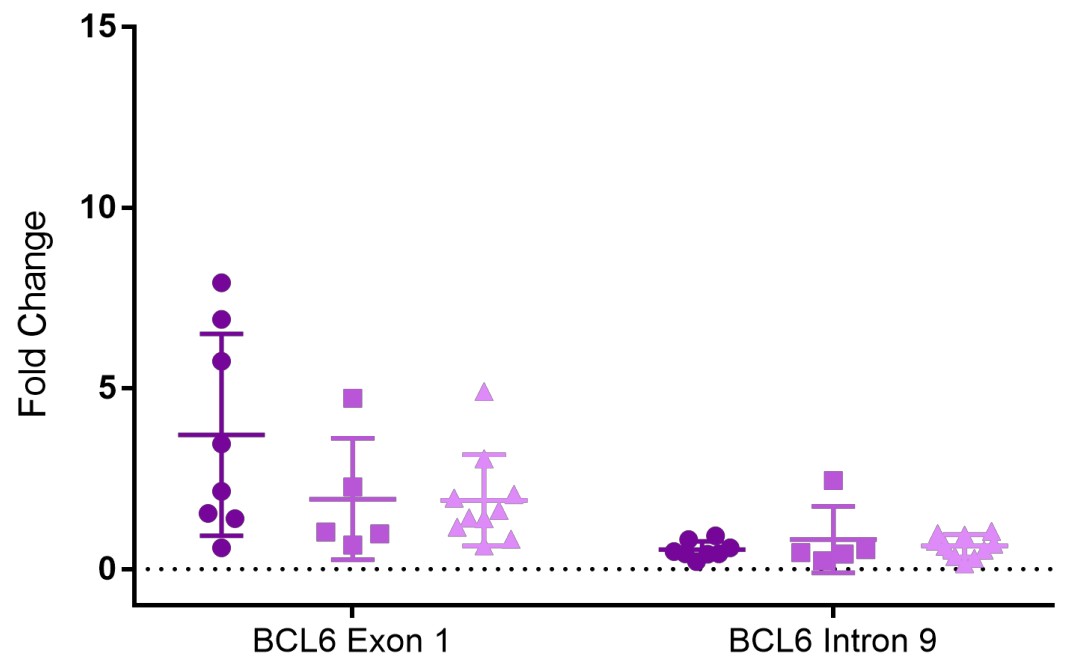

B.

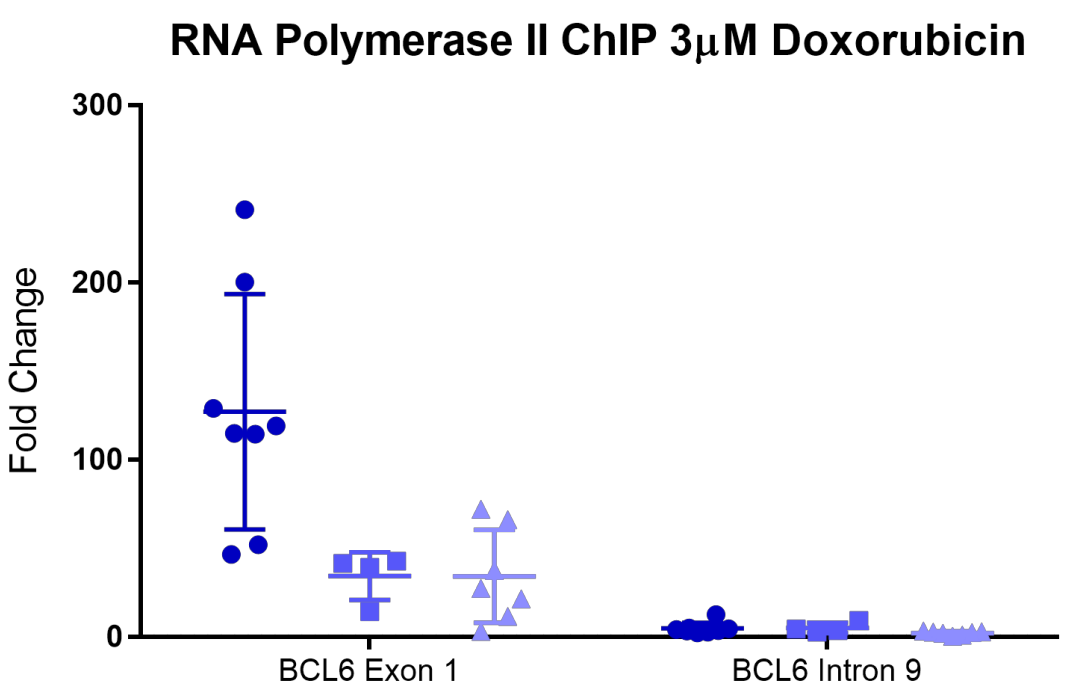

Figure 4.6: BCL6 and RNA Polymerase II ChIP on doxorubicin treated cells shows reproducible positive and negative controls. LN18 cells were treated with $3 \mu \mathrm{M}$ doxorubicin and harvested 8 or 48 hours after treatment, or left untreated, before ChIP was performed. qPCR was performed for all samples for BCL6 Exon 1 (positive control) and BCL6 intron 9 (negative control) (A) qPCR for BCL6 ChIP. (B) pPCR for RNA polymerase II ChIP. Fold change values calculated using $\Delta \mathrm{Ct}$ method with $\beta$-actin as control. Long horizontal lines represent mean \pm standard deviation of at least 4 independent experiments. 
In Figure 4.7 BCL6 and RNA polymerase II ChIP on doxorubicin treated LN18 cells is shown for candidate BCL6 target genes TARS, CCND2, and ATR, which were identified as candidates in the preliminary experiments conducted in the Melnick lab. In Figure 4.7A BCL6 was bound to TARS in untreated cells to a greater degree than in either the $8 \mathrm{~h}$ or $48 \mathrm{~h}$ doxorubicin treated time-points ( $2.21 \pm 1.22$ fold vs. $1.89 \pm 1.67$ and $1.25 \pm 0.853$ fold respectively). BCL6 binding of $C C N D 2$ and $A T R$ was low for untreated and the two doxorubicin treatment timepoints. In Figure 4.7B, RNA polymerase II was highly bound ( $86.0 \pm 40.6$ fold) to TARS in untreated LN18 cells. RNA polymerase II binding to TARS seemed to have dropped with both 8 and 48 hours doxorubicin treatment (20.9 \pm 7.31 and 19.6 \pm 16.5 fold respectively). RNA polymerase II was not bound to CCND2 in untreated and 8 and 48 hours doxorubicin treated cells. RNA polymerase II was

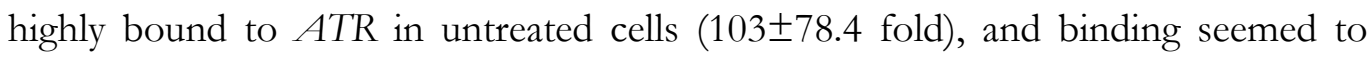
have dropped with 8 and 48 hours doxorubicin treatment $(28.1 \pm 13.3$ and $21.3 \pm 16.4$ fold respectively). 
A.

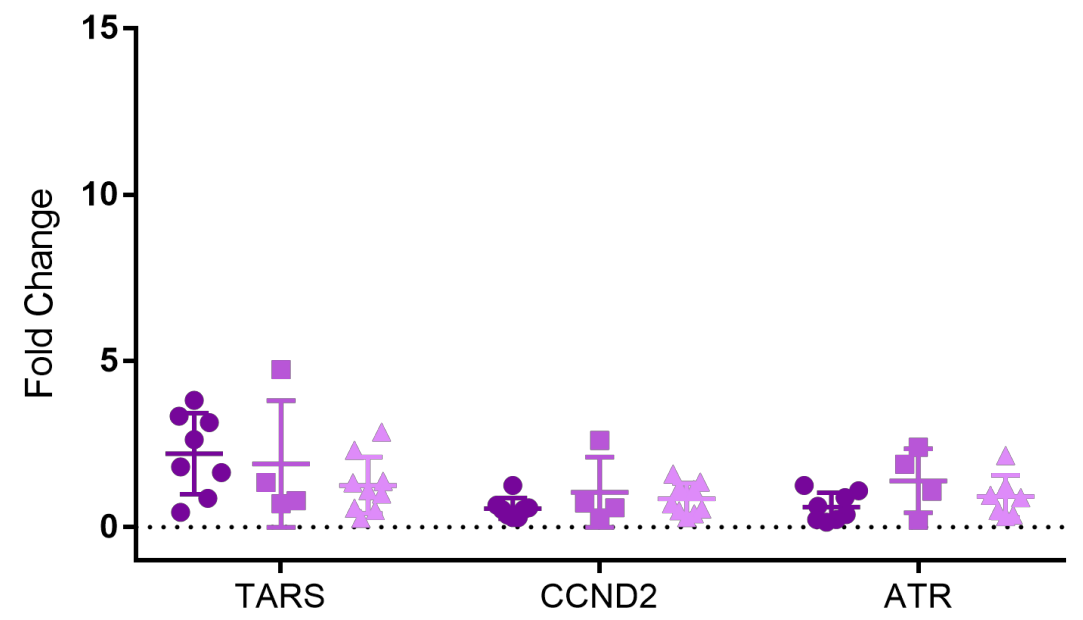

B.

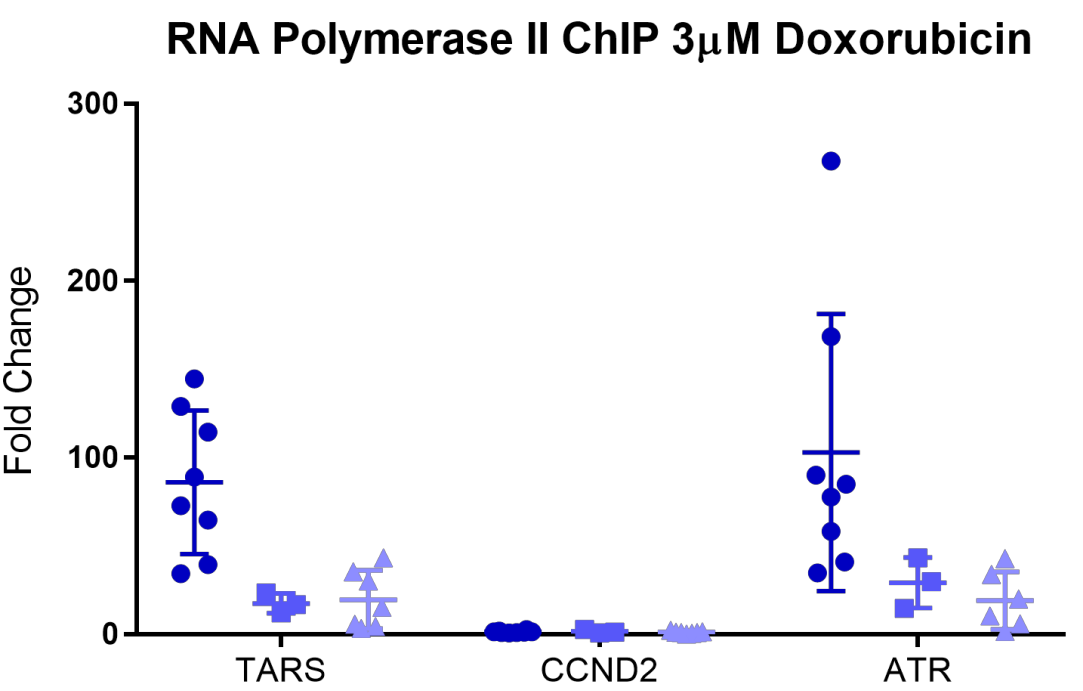

Figure 4.7: Binding of BCL6 at BCL6 Target Genes does not significantly change with doxorubicin treatment. LN18 cells were treated with $3 \mu \mathrm{M}$ doxorubicin and harvested 8 or 48 hours after treatment, or left untreated, before ChIP was performed. qPCR was performed for all samples for TARS, CCND2, and ATR. (A) qPCR for BCL6 ChIP. (B) qPCR for RNA Polymerase II ChIP. Fold change values calculated using $\Delta \mathrm{Ct}$ method with $\beta$-actin as control. Long horizontal lines represent mean \pm standard deviation of at least 4 independent experiments. 


\subsection{4 qChIP on Radiation treated LN18 cells}

Irradiation appeared to have an effect on BCL6 activity, so we wanted to investigate if BCL6 was binding to the target genes investigated with doxorubicin treated cells. LN18 cells were treated with 10 Gy ionising radiation and harvested either 8 hours or 48 hours after treatment. The untreated data is the same as presented alongside the doxorubicin data in the previous two figures.

In Figure 4.8 BCL6 ChIP and RNA polymerase II ChIP on LN18 cells treated with 10 Gy irradiation and harvested either 8 or 48 hours after treatment is shown. In Figure 4.8A BCL6 appeared to be bound to BCL6 Exon 1 fairly consistently in both time-points of irradiated cells ( 8 h $2.30 \pm 1.55$ fold and 48 h $2.65 \pm 0.525$ fold) and to a similar level as in untreated cells (3.71 \pm 2.79 fold). BCL6 Intron 9 worked well as a negative control, with no binding seen in the irradiated cells. In Figure 4.8B RNA polymerase II binding with both 8 and 48 hours post-irradiation $(65.7 \pm 41.2$ and $83.5 \pm 11.4$ fold respectively) was high and similar to the untreated cells (127士66.4 fold). RNA polymerase II was not bound to BCL6 Intron 9 in irradiated cells, which further validated this as a negative control. 
A.

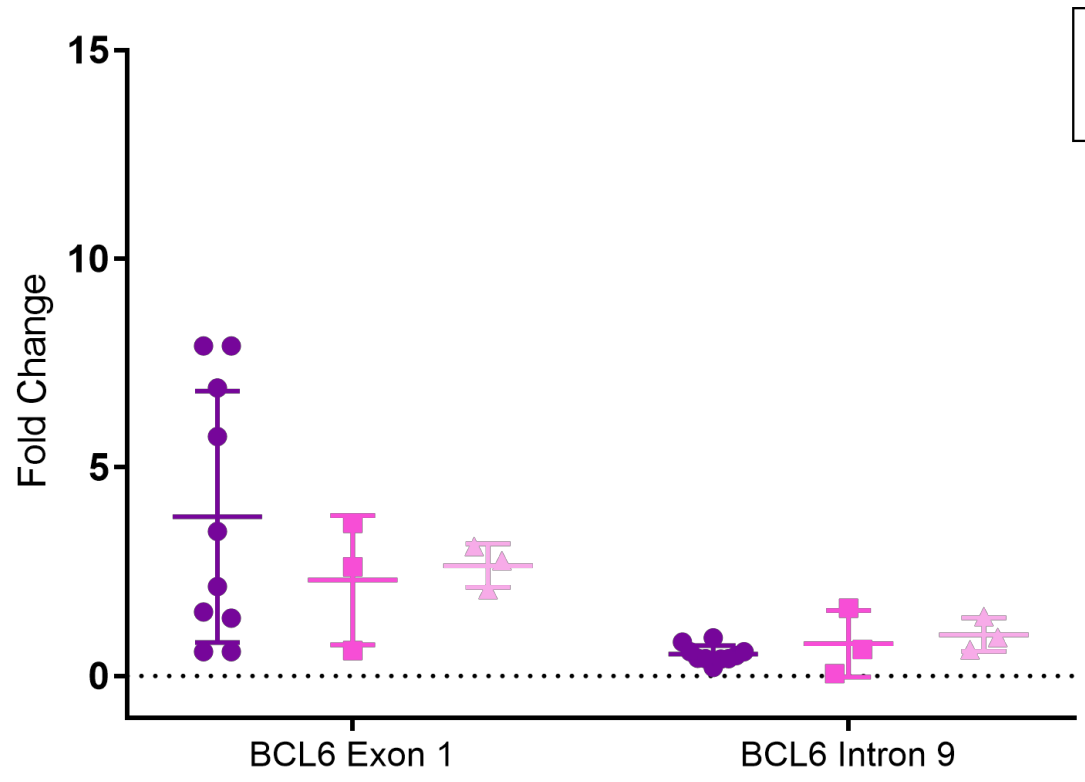

B.

RNA Pol II ChIP 10Gy Irradiation

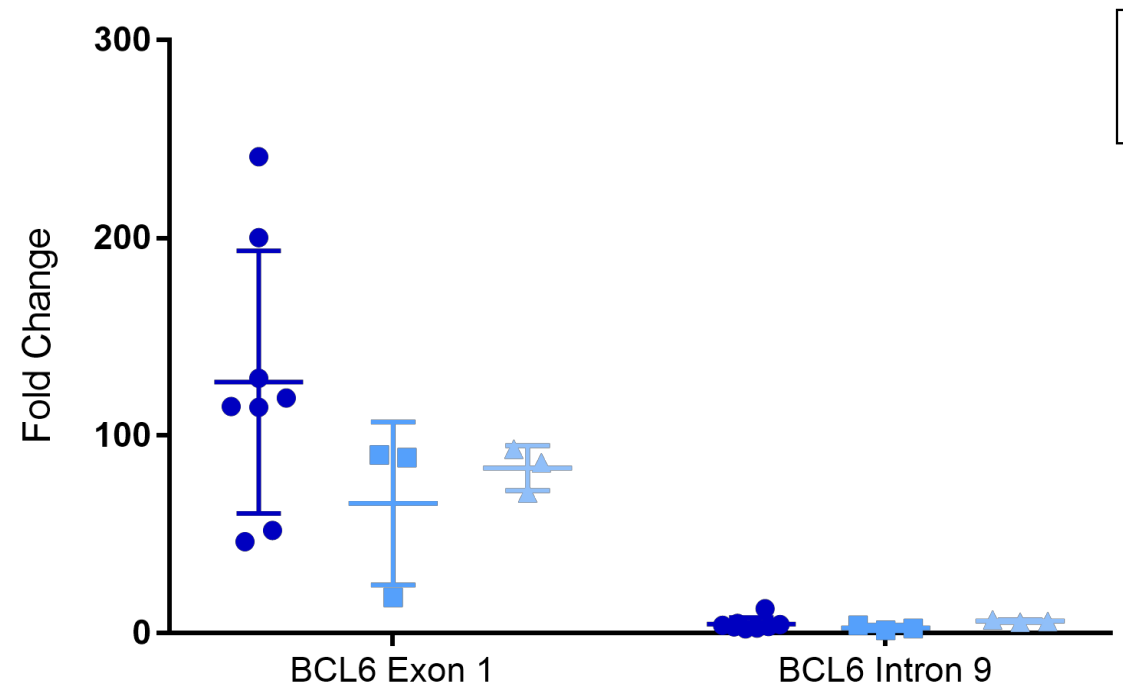

- Untreated

- 8h IR 48h IR

Figure 4.8: BCL6 and RNA Polymerase II ChIP on radiation treated cells shows reproducible positive and negative controls. LN18 cells were treated with $10 \mathrm{~Gy}$ irradiation and harvested 8 or 48 hours after treatment, or left untreated, before ChIP was performed. qPCR was performed for all samples for BCL6 Exon 1 (positive control) and BCL6 intron 9 (negative control) (A) qPCR for BCL6 ChIP. (B) pPCR for RNA polymerase II ChIP. Fold change values calculated using $\Delta \mathrm{Ct}$ method with $\beta$-actin as control. Long horizontal lines represent mean \pm standard deviation of at least 3 independent experiments. 
In Figure 4.9 BCL6 and RNA polymerase II ChIP is shown for candidate BCL6 target genes TARS, CCND2, and ATR on irradiation treated LN18 cells. In Figure 4.9A BCL6 appears to be bound to TARS in both 8 and 48 hours post-irradiation cells $(2.19 \pm 2.13$ and $2.14 \pm 0.828$ fold respectively) to a similar level as seen in the untreated cells (2.21 \pm 1.22 fold). BCL6 binding of CCND2 and ATR was low in both 8 and 48 hours post-irradiation cells and similar to the untreated cells. In Figure 4.9B RNA polymerase II was highly bound to TARS in both 8 and $48 \mathrm{~h}$ post irradiation cells $(95.1 \pm 71.5$ and $61.1 \pm 28.7$ fold respectively) and to a similar level seen in untreated cells $(86.0 \pm 40.6$ fold). RNA polymerase II was not bound to CCND2 in 8 or $48 \mathrm{~h}$ post-irradiation cells. RNA polymerase II was highly bound to ATR in both 8 and $48 \mathrm{~h}$ post-irradiation samples (121 \pm 86.2 and $85.4 \pm 38.5$ fold respectively) and to a similar level as untreated cells (103 \pm 78.4 fold). 
A.

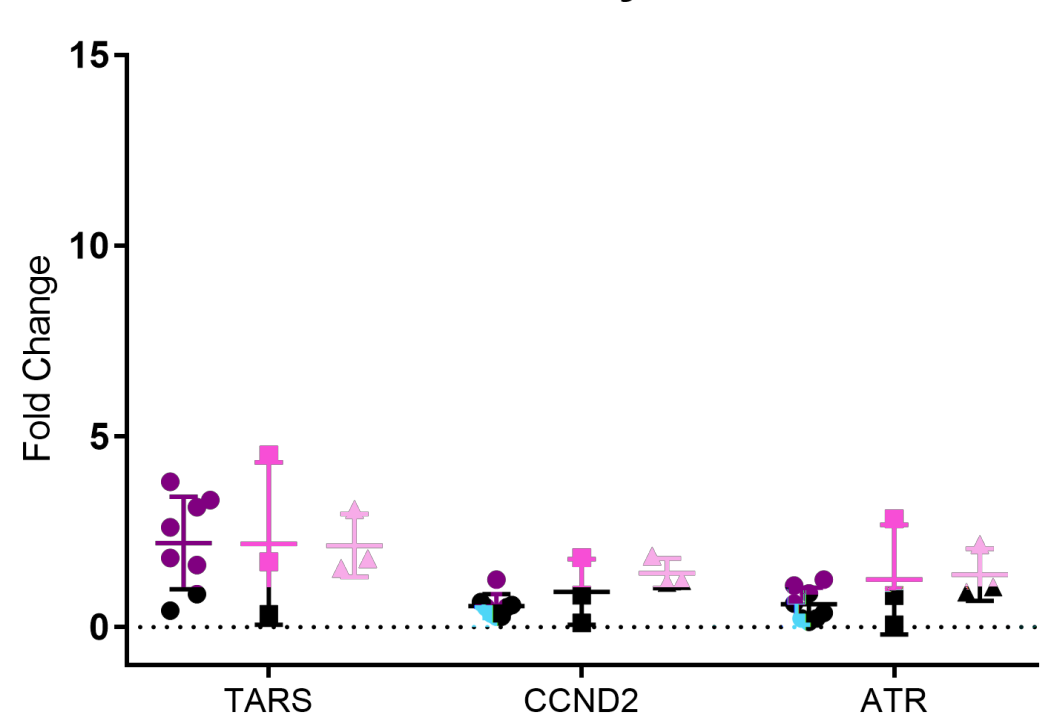

B.

\section{RNA Pol II ChIP 10Gy Irradiation}

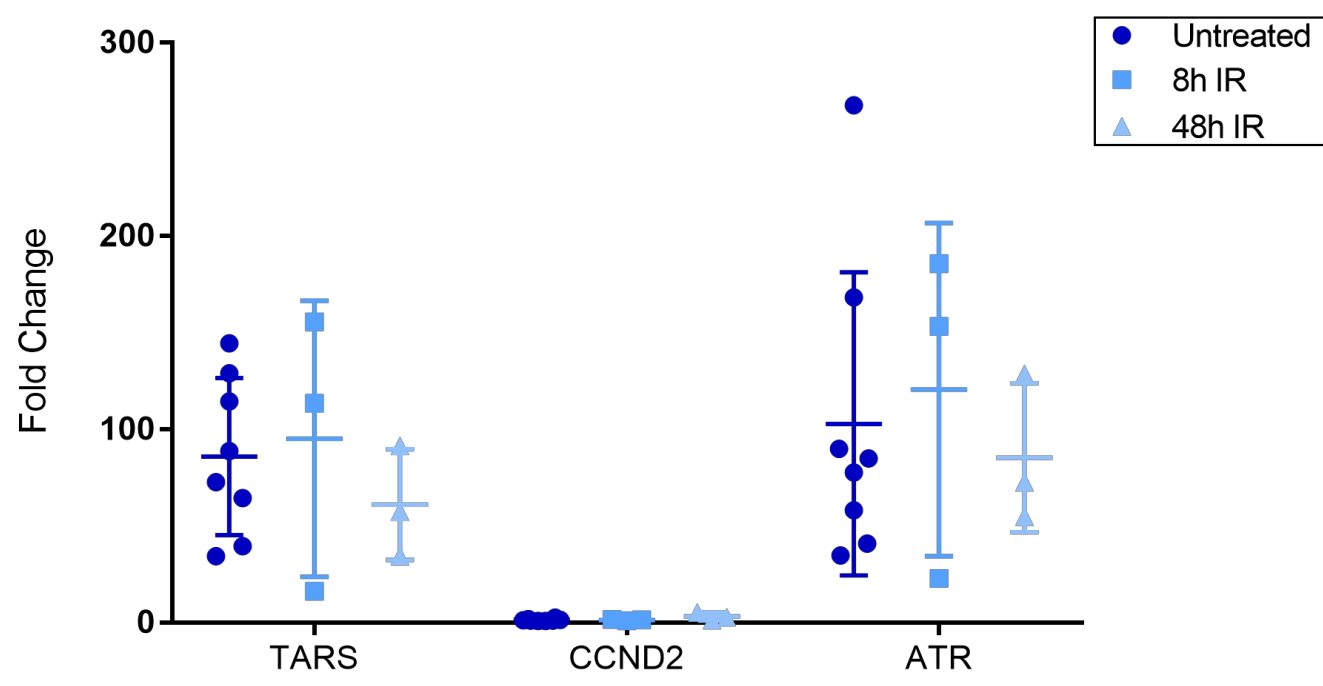

Figure 4.9: Binding of BCL6 at BCL6 Target Genes does not significantly change with radiation treatment. LN18 cells were treated with 10 Gy irradiation and harvested 8 or 48 hours after treatment, or left untreated, before ChIP was performed. qPCR was performed for all samples for TARS, CCND2, and ATR. (A) qPCR for BCL6 ChIP. (B) qPCR for RNA Polymerase II ChIP. Fold change values calculated using $\Delta \mathrm{Ct}$ method with $\beta$-actin as control. Long horizontal lines represent mean \pm standard deviation of at least 3 independent experiments. 


\subsection{Summary}

BCL6 is a transcriptional repressor that exerts this activity by binding to DNA and recruiting co-repressors and HDAC3 to shut down gene expression. Work completed in Chapter 3 showed that BCL6 may not in fact be a transcriptional repressor in GBM and subsequently in this chapter it was investigated if BCL6 was able to bind DNA, and specifically well-known BCL6 target genes.

When BCL6 is overexpressed in LN18 cells it is able to bind an isolated single consensus sequence and form complexes with its co repressors BCOR, NCOR, and SMRT as expected. However, when looking at endogenous or doxorubicin induced BCL6, it was unable to bind its consensus sequence. This may be because there was only one BCL6 binding sequence on a short oligonucleotide, which would have reduced the chances of endogenous BCL6 interacting with its binding site, whereas when BCL6 was overexpressed there was an excess of BCL6 so it is more likely it would interact with the one binding site that was on the oligonucleotide. Given the results seen with the ChIP experiments, it is probable that endogenous BCL6 in LN18 cells interacts with its binding site, but the oligonucleotide used to assess this in the EMSA needed to have multiple copies of the binding site for this to be seen.

This work was followed up with a ChIP assay to further investigate if BCL6 was bound to well known target genes under different treatment conditions. The ChIP experiments were performed initially in the Melnick lab in order to establish the ChIP technique in LN18, and due to differing technologies being available, a number of adaptations had to be made to be able to do the technique in the McConnell lab. One of the adaptations was the sonication. In the Melnick lab the sonication was completed with the Covaris E220 ultrasonicator, which is designed for DNA shearing and produces very accurate and consistent shearing of the DNA. In the McConnell lab the sonication was completed with a SONOPULS mini20 ultrasonic homogeniser, which is not designed specifically for DNA shearing, and much optimisation was required to shear the DNA accurately and consistently with this machine. The difference between these machines has likely introduced some differences between the experiments completed in the two laboratories. 
Another potentially major change that was made to the protocol was changing the control antibody from a polyclonal non-specific rabbit IgG to a monoclonal mouse $\beta$-actin. This was done as it was believed to be a better control to use, since actin is predominantly cytoplasmic, and this control would also match well with the RNA polymerase II antibody used, as this was also a mouse monoclonal antibody. Since the completion of these experiments research came to light that shows that actin can be a nuclear protein, and in fact appears to co-localise with RNA polymerase II (208-210). The $\beta$-actin precipitation was intended as a control with no binding to DNA. If actin was bound to DNA, subtracting this from the BCL6 signal would inappropriately decrease the apparent level of BCL6 binding. This may explain why the results from BCL6 ChIP experiments in the Melnick lab were on a different scale to the experiments completed in the McConnell lab: 5-23 fold enrichment in the Melnick lab versus 1-5 fold enrichment in the McConnell lab.

However, considering that actin has been shown to co-localise with RNA pol II, it would be expected that if there were significant amounts of actin cross-linked to DNA, subtracting the actin ChIP signal from the RNA pol II ChIP would show little or no enrichment. This was not the case, as massive enrichment of binding was found with RNA pol II ChIP on most loci investigated. The use of actin as a negative control does not completely explain why significant enrichment at CCND2 and ATR was not seen with BCL6 ChIP, and it is more likely that BCL6 has low binding of these genes in LN18 cells.

In the McConnell lab, it was observed that BCL6 was bound to BCL6 Exon 1 and TARS in untreated LN18 cells, and this pattern was similar in radiation treated cells. Doxorubicin treated cells had reduced BCL6 binding of BCL6 Exon 1 compared to untreated, with 1.5 fold enrichment with doxorubicin versus 3.7 fold enrichment in untreated cells. Similar binding was seen at TARS with doxorubicin compared to untreated, with 1.2 fold enrichment with doxorubicin versus 2.2 fold enrichment in untreated cells. Given that radiation treated cells have similar levels of BCL6 expression to untreated cells, it made sense that the binding of BCL6 would be similar across all genes for both radiation treated and untreated cells. It is interesting that a decrease in binding of BCL6 Exon 1 was seen with doxorubicin, as there was an increase in BCL6 protein with this treatment. This may indicate that BCL6 DNA 
binding changes with treatment, and has perhaps has moved to different target genes not investigated in this study. ChIP sequencing would reveal what these targets would be.

RNA polymerase II was bound to BCL6 Exon 1, TARS, and ATR in untreated cells, and this binding was reduced by doxorubicin, but not by radiation, which further showed the difference between these two treatments. This could indicate that there is some kind of non-specific "dox effect" on RNA polymerase II binding, rather than a BCL6 specific effect on transcription of these genes in the doxorubicin treated cells. 


\section{Effect of BCL6 on Gene Expression in Glioblastoma}

\subsection{Introduction}

BCL6 is a transcription factor, and affects the transcription of genes. In most settings, it is known as a transcriptional repressor, which means it shuts down the expression of genes. It does this through the recruitment of the co-repressor proteins BCOR, NCOR, and SMRT, which then recruits HDAC3. HDAC3 deacetylates the histones that the DNA is attached to. This reduces DNA availability for transcription by RNA pol II, shutting down gene expression.

Transcriptional repressor activity is essential for BCL6 function in B-cells. During maturation of germinal centre B-cells, their genome undergoes somatic hypermutation and class switch recombination to create cell-unique specific antibody genes. This is crucial for the development of a wide immune repertoire. While the DNA breaks and is reorganised, BCL6 represses a whole host of DNA damage response and cell cycle checkpoint genes, to allow the cell to survive this process. This is a key part in the development of lymphoma, where translocations of other anti-apoptotic genes like BCL2 in combination with BCL6 expression lead to the over proliferation of GC B-cells, resulting in lymphoma. However, BCL6 is not always a transcriptional repressor, and has been shown in other settings to activate gene expression $(187,206)$.

BCL6 is expressed in response to DNA damage in GBM, but it is not clear what effect BCL6 has on gene expression in GBM. Earlier results presented in this thesis suggest that BCL6 can bind DNA in GBM and therapy-induced BCL6 is not a transcriptional repressor in response to DNA damage, but may be a transcriptional activator.

It is not yet clear whether BCL6 expression is important to the DNA damage response and subsequent therapy resistance in GBM. If BCL6 plays an important role in GBM cells, BCL6 expression is likely to have a whole host of effects on the transcriptional profile of the cells. In order to take a broad look at the transcriptional effect of BCL6 in GBM cells, RNA sequencing was undertaken on 
cells that had been treated with BCL6 inhibitor FX1, alongside the vehicle control. RNA sequencing is an unbiased approach to look at the transcriptome of cells, and changes in the transcriptome seen with FX1 treatment will allow the identification of genes that are affected by BCL6 in GBM cells, but it is also likely it will identify changes related to off-target effects of FX1.

RNA sequencing was also completed on GBM cells treated with DNA damaging therapies doxorubicin and IR, alongside the untreated control. Changes in transcription seen with these DNA damaging agents will show the transcriptional program used by GBM cells in response to these agents, and should highlight differences and similarities between these two treatments.

Comparison between the cells that have sustained DNA damage and the cells that have been treated with BCL6 inhibitor FX1 should expose how the genes and pathways affected by BCL6 are also altered in response to DNA damage, but this may also include pathways that are not related to BCL6, due to the potential offtarget effects of FX1.

\subsection{Aims}

The aims of this chapter are to observe global gene expression changes in LN18 cells in response to BCL6 inhibition, and compare to treatment with doxorubicin or ionising radiation.

\subsection{Results}

\subsubsection{Planning and preparation of RNA samples for sequencing}

RNA sequencing can be used to sequence all types of RNA that exist within a cell. The most common use of RNA sequencing is to sequence all of the mRNA transcripts expressed within a pool of cells, which produces a snapshot in time of the global gene expression of that group of cells. RNA sequencing projects must be carefully planned in order to produce data that is accurate and useful to the researcher. 
For this project, the most useful information would come from the comparison between the DNA damaging agents doxorubicin and IR and the BCL6 inhibitor FX1. Doxorubicin and IR treatment lead to increased expression of BCL6 as well as increased transcriptional activity in a BCL6-specific luciferase assay, and irradiation lead to a 3-fold increase in transcriptional activity in a BCL6-specific luciferase assay (Chapter 3, and Appendix: Fabre, Jones, et al., in revision). FX1 is a highly specific BCL6 inhibitor as it prevents the interaction between BCL6 and its co-repressors (179). FX1 has a similar $\mathrm{IC}_{50}$ in GBM cells compared to BCL6 dependent lymphoma lines (See Appendix: Fabre, Jones, et al., in revision). The comparison between the gene expression of cells treated with DNA damaging agents and FX1 would indicate genes and pathways that BCL6 affected in these cells, but it is important to approach this with caution, as it is also likely it will identify changes related to off-target effects of FX1. This information could lead future research into BCL6 in GBM, as it would give an indication of important genes/pathways to focus on, to start to determine the transcriptional function of BCL6 in GBM. Doxorubicin was used at a concentration of $3 \mu \mathrm{M}$ and ionising radiation at a dose of $10 \mathrm{~Gy}$, as these induce BCL6 expression and/or increase transcriptional activity of BCL6, as seen in chapter 3. While the $\mathrm{IC}_{50}$ of FX1 for LN18 cells was $40 \mu \mathrm{M}$ (See Appendix: Fabre, Jones, et al., in revision), treatment at this concentration led to poor quality RNA. Instead, FX1 was used at a concentration of $25 \mu \mathrm{M}$. As noted in Chapter 3, FX1 can inhibit other kinases above a concentration of $10 \mu \mathrm{M}$ particularly FAK, FGFR1 and SYK (179), which may mean that effects of FX1 seen are not necessarily related to BCL6 inhibition. All cells were collected for RNA extraction 24 hours after treatment, as this time point has been shown to capture changes in the transcription of BCL6 target genes caused by FX1 treatment in lymphoma cells (179).

RNA sequencing is highly sensitive and accurate, but can only be truly reflective of gene expression if high quality RNA is used for sequencing. RNA extraction was completed using the Zymo Quick RNA MiniPrep kit, and 3 different checks were used to determine RNA quality and quantity. First, RNA was quantified using the Qubit ${ }^{\mathrm{TM}}$ fluorometer, which uses dyes that fluoresce when they bind RNA, and the fluorescence can be measured relative to a set of standards that give accurate 
quantification. The purity of the RNA was determined by absorbance using the NanoDrop spectrophotometer, using the ratio of absorbance of the RNA at $260 / 280 \mathrm{~nm}$ and secondarily the ratio of $260 / 230$. A 260/280 ratio of 2.0 and 260/230 of 2.0-2.2 for RNA is considered to be pure. The integrity of the RNA was determined by Agilent Bioanalyzer RNA 6000 Nano chip, which calculated an RNA Integrity Number (RIN) based on how intact or degraded the RNA is. RIN is valued from 1-10 where 10 indicated completely intact RNA. All of these parameters needed to be within an acceptable range to give good quality sequencing data:

Concentration $\quad>100 \mathrm{ng} / \mu \mathrm{l}$

Absorbance $(260 / 280) \quad>1.8$

Absorbance $(260 / 230) \quad>1.5$

RNA Integrity Number $\quad>7$

In Table 5.1 RNA quality values are shown for the samples sent for library preparation and sequencing. All of these values are well above the limits set by NZGL, and close to the values that would indicate high concentration, pure, and intact RNA. In Figure 5.1 pictures of the cells harvested for RNA are shown. The three treatments (doxorubicin, IR and FX1) have an effect on the morphology or division of the cells as compared to their respective controls. Doxorubicin appears to have reduced the division of the cells as compared to the untreated control and the cells were flatter and larger. Despite this effect of doxorubicin on overall cell health, good quality RNA was obtained from these samples (Table 5.1). Irradiation did not affected cell division very much, but the cells were morphologically changed, appearing flat and elongated compared to the untreated control. The FX1 treated cells are flatter and larger compared to the DMSO control, but there was not any obvious effect on cell division. 


\begin{tabular}{|l|r|r|r|r|}
\hline & ng/ $\mathbf{\mu l}$ & A (260/280) & A (260/230) & RIN \\
\hline Untreated 1 & 1264 & 2.05 & 2.1 & 8.7 \\
\hline Untreated 2 & 1420 & 2.04 & 2.02 & 9.3 \\
\hline Untreated 3 & 1624 & 2.03 & 2 & 8.4 \\
\hline $\mathbf{3} \boldsymbol{\mu M}$ Doxorubicin 2 & 1252 & 2.04 & 2.15 & 10 \\
\hline $\mathbf{3} \boldsymbol{\mu M}$ Doxorubicin 3 & 956 & 2.05 & 2.11 & 10 \\
\hline $\mathbf{3} \boldsymbol{\mu M}$ Doxorubicin 4 & 1848 & 2.05 & 2.13 & 10 \\
\hline $\mathbf{1 0}$ Gy Irradiation 2 & 1784 & 2.05 & 2.15 & 10 \\
\hline $\mathbf{1 0}$ Gy Irradiation 3 & 1908 & 2.05 & 1.94 & 9.9 \\
\hline $\mathbf{1 0}$ Gy Irradiation 4 & 2640 & 2.05 & 1.76 & 10 \\
\hline $\mathbf{2 5} \boldsymbol{\mu M}$ FX1 2 & 122 & 2.03 & 2.22 & 9.2 \\
\hline $\mathbf{2 5} \boldsymbol{\mu M}$ FX1 3 & 336 & 2.04 & 2.25 & 9.3 \\
\hline $\mathbf{2 5} \boldsymbol{\mu M}$ FX1 4 & 370 & 2.1 & 2.23 & 9.6 \\
\hline DMSO 1 & $\mathbf{6 8 2}$ & 2.03 & 2.22 & 9.4 \\
\hline DMSO 2 & 526 & 2.02 & 2.17 & 9.4 \\
\hline DMSO 3 & 750 & 1.98 & 2.11 & 9.2 \\
\hline
\end{tabular}

Table 5.1 RNA quality of samples used for sequencing. Sample names reflect sample numbers used, 4 replicates were produced for each treatment and the 3 best samples were chosen for sequencing. Concentration was measured using the Qubit fluorometer, absorbance was measured using the Nanodrop spectrophtometer and RNA Integrity Number (RIN) was calculated by the Agilent Bioanalyzer 2100. 
A.

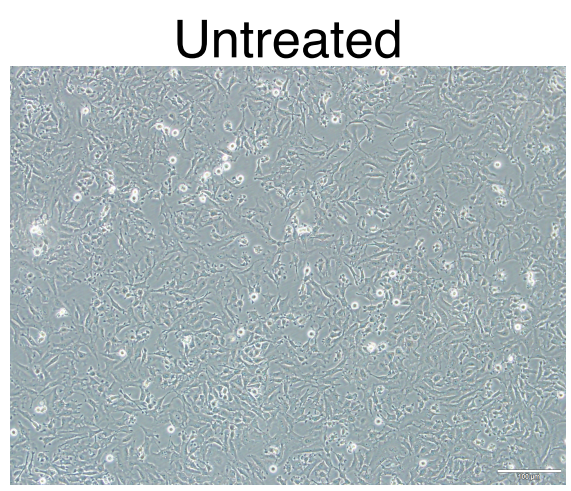

C.
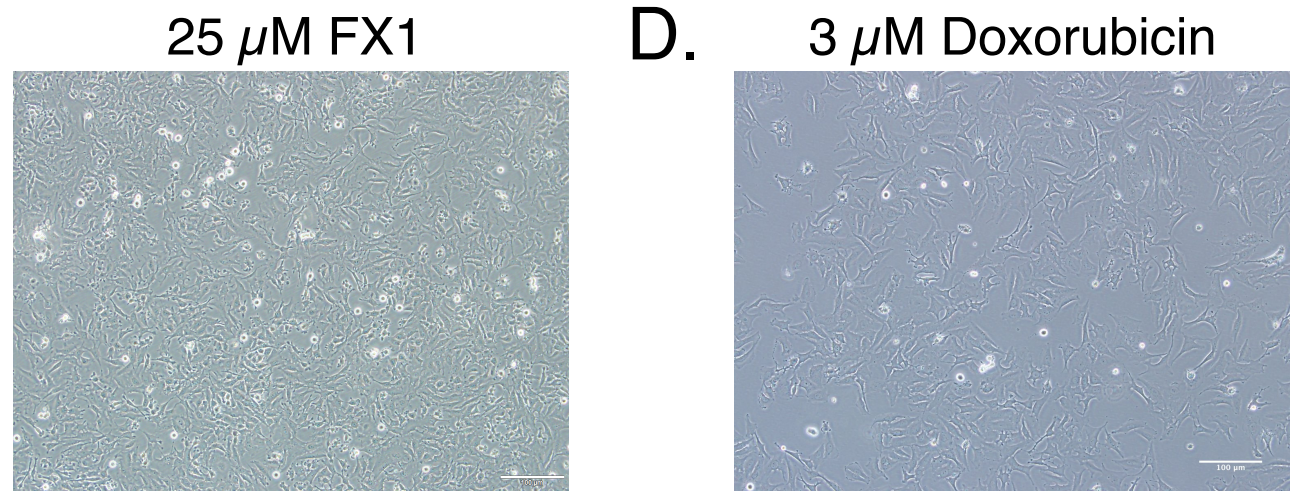

D.

B.

DMSO

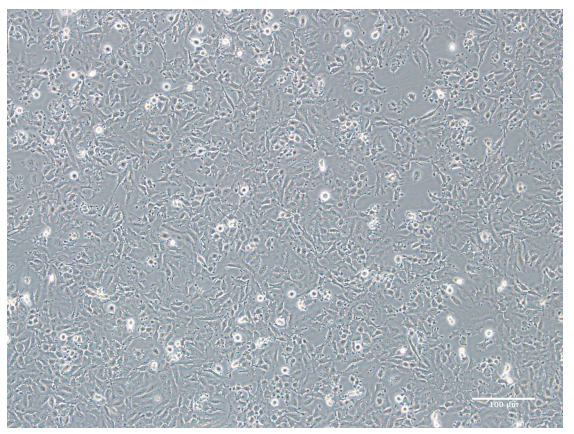

$\mu \mathrm{M}$ Doxorubicin

E. 10 Gy Irradiation

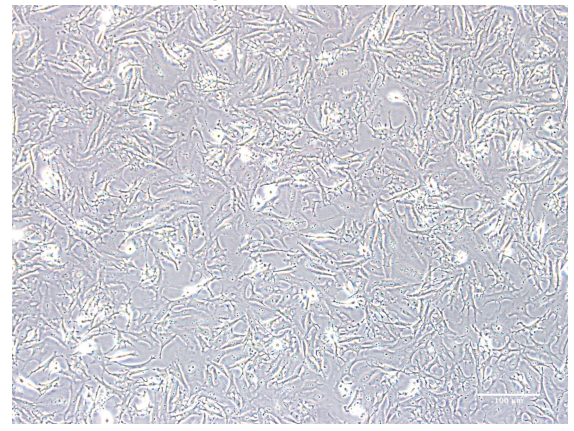

Figure 5.1 Pictures of LN18 cells used for RNA sequencing just before harvest for RNA. (A) Untreated cells. (B) Cells treated with volume equivalent DMSO to FX1 treated cells. (C) Cells treated with $25 \mu \mathrm{M}$ FX1. (D) Cells treated with $3 \mu \mathrm{M}$ doxorubicin. (E) Cells treated with $10 \mathrm{~Gy}$ irradiation. Scale bars on all pictures indicate $100 \mu \mathrm{m}$. 


\subsubsection{Quality of RNA Sequencing}

The library preparation and sequencing was completed by NZGL, and Dinindu Senanayake completed initial quality control (QC), mapping and differential expression on the data received. Samuel Lee performed the entire pipeline from initial QC through to differential expression and produced the graphs presented for analysis under the guidance of Nicole Jones.

FastQC was used to examine the quality of the raw sequencing reads by producing a series of statistics that allow researchers to quickly identify any problems with the raw sequencing data (188). Trimmomatic was used to remove overly represented adapter sequences and low quality reads (189). Once the data had been trimmed and passed the FastQC tests it was pseudoaligned and quantified with Kallisto (190), and the individual transcript abundances produced by Kallisto were collapsed to the gene level using the $\mathrm{R}$ package tximport (192). These gene level abundances were used for all downstream analysis presented in this thesis. 


\subsubsection{Principal Component Analysis}

Principal component analysis (PCA) is used to reduce the dimensionality of multidimensional data-sets to allow for visualisation of the variance between datasets, without categorical information. In the case of PCA for RNA-sequencing data, each gene in a data-set is a dimension, which means there are thousands of dimensions in any one data-set. It makes sense to try to reduce this dimensionality to start to visualise the data. The PCA will also inform whether there are differences between different experimental conditions. In a PCA, clustering of samples together indicates they are similar, and the further apart a data point is from others, the more dissimilar it is. The first few components are usually the only ones considered for PCA, as they account for the majority of the variance of a data-set.

In Figure 5.2, PCA plots are shown for the first three principal components of the RNA sequencing data-set. Each point represents one RNA sample, and as every treatment/control was performed in triplicate, this results in a total of 15 sets of sequencing data. In Figure 5.2A it can be observed that PC1 on the x-axis accounts for $91 \%$ of the variance of the samples, with the doxorubicin treated data points clustering together tightly, but as an outlier far from the other treatments and controls. This highlights that the effect of doxorubicin on the cells was vastly different to that of the other treatments used. The IR, FX1, untreated and DMSO data points all cluster together nicely within each treatment group, but generally are similar for PC1. PC2 along the y-axis of Figure 5.2A accounts for 5\% of the variance between the data points and shows that each treatment group is different, with the untreated and DMSO controls clustering together in the middle, and the FX1 and IR groups being quite distant from each other along this axis. DMSO and FX1 are close together as are the untreated and IR data points, which makes sense as these are their respective controls. In Figure 5.2B PC2 and PC3 are shown on the $\mathrm{x}$ - and $\mathrm{y}$-axis respectively. The doxorubicin data points cluster tightly in the middle of PC2, almost exactly between the FX1 and IR data points, which further demonstrates the difference between each of these treatments. PC3 accounts for $2 \%$ of the total variance, and along PC3 (y-axis) the treatments and controls seem to separate further, with doxorubicin, IR and FX1 clustering at the lower half of PC3 and untreated and DMSO clustering at the upper half of PC3. The FX1 data points seem to vary the most compared to the other treatments and controls, but they still 
cluster together, and there is no overlap between any of the clusters for each treatment or control data points. Together, these two plots in Figure 5.2 account for $98 \%$ of the variance of the data points, and overall they show good clustering of each of the 3 data points for each treatment/control, which indicates that the treatment/control replicates are more similar to each other than to other treatment/control groups. These plots indicate that there is only a small amount of variation between the replicates and subsequently these are likely to be good representations of what is happening within the cells. The variation observed between groups is most likely due to the different treatments used. 
A.

PC1 v PC2

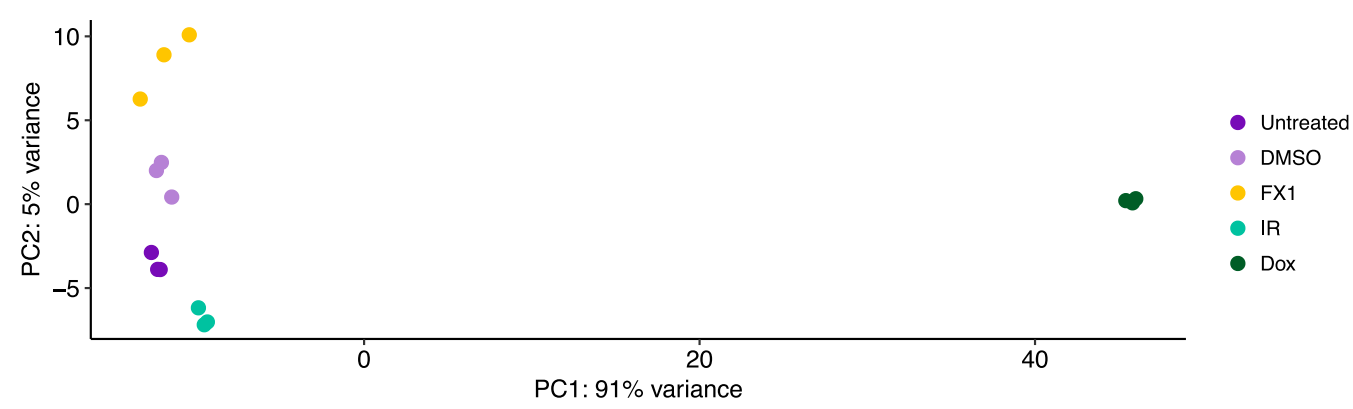

B.

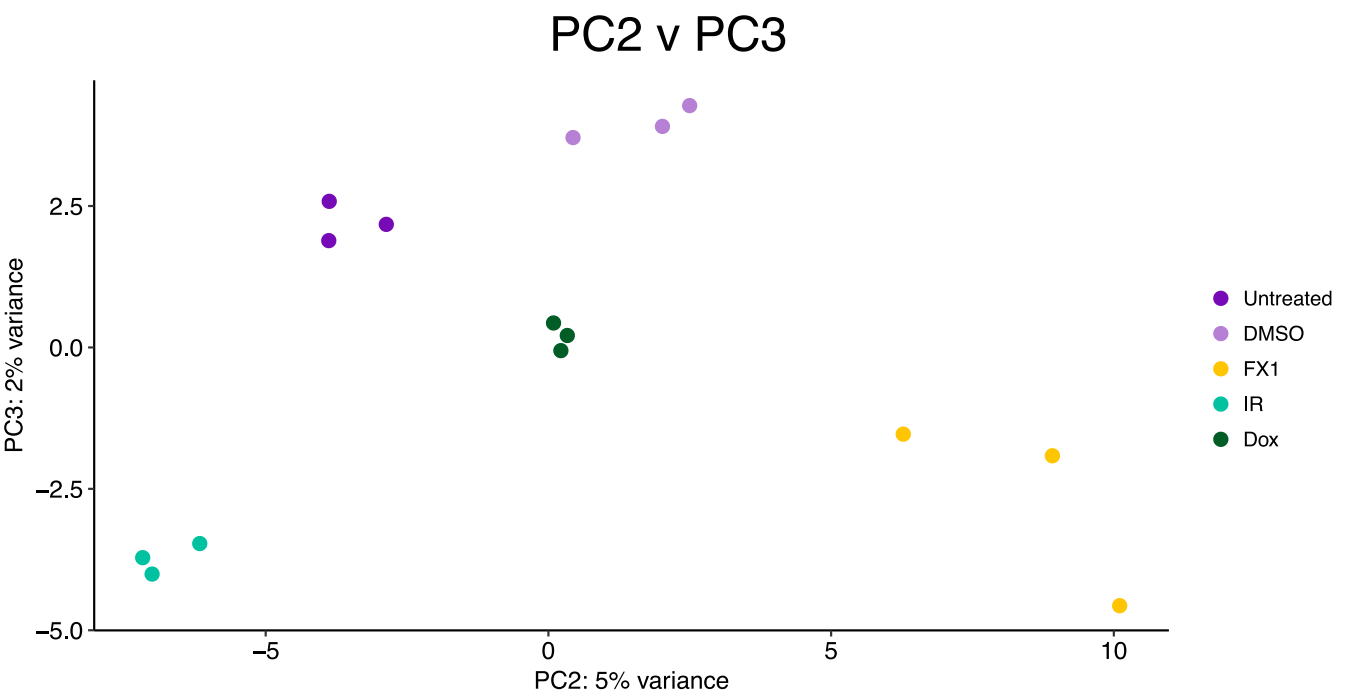

Figure 5.2: Principal Component Analysis of the RNA sequencing data-set. (A) Principal component 1 (x-axis) v principal component 2 (y-axis). (B) Principal component 2 (x-axis) v principal component 3 (y-axis). Scale for both graphs is indicative of variance, and $\%$ variance is the total amount of variation of the data set that can be explained by the principal component. For both graphs, (Dox) doxorubicin and (IR) ionising radiation. 


\subsubsection{Differential Expression Analysis}

Differential expression analysis (DEA) was performed on the three treatment datasets, which is where the treatments are compared to their respective controlsDMSO for FX1, and untreated for doxorubicin and IR. This gives a final result of up- and down-regulated genes as compared to the controls. DEA was performed using the DESeq2 method (211), and a gene was defined as differentially expressed if it had a $\log 2$ fold change $(\log 2 \mathrm{fc})<-1.5,>1.5$ and a Benjamini Hochberg adjusted p-value $<0.05$ (padj). One of the most common plots to use to examine this kind of data from a "birds eye view" is a volcano plot. Volcano plots show the relationship between the padj and $\log 2 \mathrm{fc}$, as they present the data as the $-\log 10$ of padj on the $\mathrm{y}$ axis, and the $\log 2 \mathrm{fc}$ on the $\mathrm{x}$-axis. The data points (in this case the genes) are represented as dots, and the most statistically significant genes with the largest fold change values will be located in the top left- and right-hand corners of this type of plot. The left-hand side of the plot indicates down-regulated genes, the right-hand side of the plot indicates up-regulated genes. As a result, volcano plots can give an idea of whether treatment caused an overall increase or decrease in global gene expression.

In Figure 5.3 volcano plots of the FX1 vs. DMSO, doxorubicin vs. untreated, and ionising radiation vs. untreated are shown. Figure 5.3A shows the volcano plot for the FX1 vs. DMSO genes, and the genes that reach the significance cut-offs are represented as yellow dots. There may be a slight skew towards overall decrease in gene expression with FX1 treatment (42 up, 64 down).

Figure 5.3B shows the volcano plot for the doxorubicin vs. untreated genes, and the genes that reach the significance cut-offs are represented as dark green dots. Doxorubicin had a profound effect on gene expression with large numbers of genes both up and down-regulated in response to treatment, and there are more significantly up-regulated genes than down-regulated (3228 up, 934 down). This massive effect of doxorubicin on gene expression is unsurprising, given how vastly different the doxorubicin data was shown to be in the principal component analysis (Figure 5.2). 
Figure 5.3C shows the volcano plot for the ionising radiation vs. untreated genes, and the genes that reach the significance cut-offs are represented as turquoise dots. The dramatic changes in gene expression seen with doxorubicin are not seen with ionising radiation, which highlights the differences in cellular response to these two DNA-damaging agents. Though the padj and $\log 2 \mathrm{fc}$ values observed are overall smaller than those observed in the doxorubicin data, there are more genes significantly up-regulated than down-regulated (103 up, 28 down). 

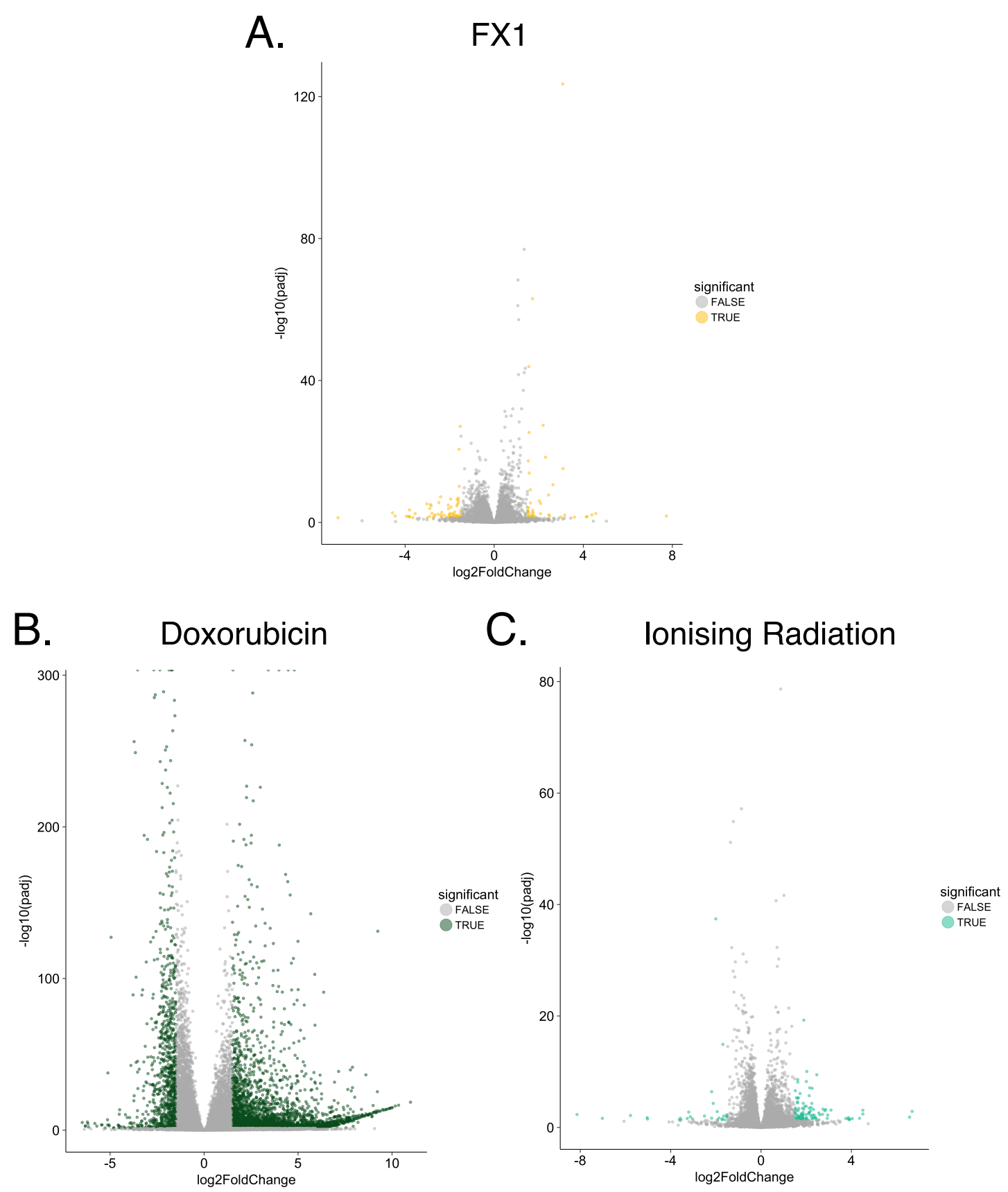

Figure 5.3 Volcano plots of differential expression. Volcano plots with $\log 2$ fold change on the $\mathrm{x}$-axis and $-\log 10$ Benjamini Hochberg adjusted $\mathrm{p}$-value on the $\mathrm{y}$-axis of differential expression for three treatment groups as compared to their respective controls. (A) FX1 treated cells as compared to DMSO treated cells. (B) Doxorubicin treated cells as compared to untreated cells. (C) Ionising radiation treated cells as compared to untreated cells. For all graphs, grey dots represent genes that don't meet the significance threshold and coloured dots represent genes that do. The significance threshold used here is $\log 2$ fold change $<-1.5,>1.5$ and a Benjamini Hochberg adjusted p-value $<0.05$. 
Another way of visualising the differential expression analysis is to look at how many genes each data-set has in common. This can be done using a Venn diagram. Figure 5.4A is a 3-way Venn diagram that shows the number of genes that have reached the significance cut-offs used for DEA for each data-set and the number of genes in common between these data-sets.

FX1 and IR treated data-sets have similar numbers of unique genes (54 and 53 respectively), whereas there are a high number of unique genes in the doxorubicin data-set (4036). The high number of unique genes in the doxorubicin data-set further reflects the dramatic effect that doxorubicin has on global gene expression as shown by the PCA (Figure 5.2) and volcano plot (Figure 5.3A).

Nearly half of the total significant genes in the FX1 data-set are also found in common with the doxorubicin data-set (51 out of 106 total). This is not the case with IR, as only $\sim 3 \%$ of the significant genes ( 3 out of 106) in the FX1 data-set are in common with the IR data-set. The IR and doxorubicin data-sets have more in common (77 genes) than FX1 and IR (3 genes), which is likely related to the fact that doxorubicin and IR both cause DNA damage. There are 2 genes that are differentially expressed in all conditions, which are naked cuticle homolog 1 (NKD1) and yippee like 1 (YPEL1). NKD1 is a member of the NKD (naked cuticle) family of genes, which regulate the Wnt signalling pathway $(212,213)$. NKD1 is up-regulated with doxorubicin treatment and down regulated with FX1 and IR. The function of YPEL1 has yet to be fully elucidated, but it has been shown to induce an epithelial-like transition in NIH3T3 cells (214). YPEL1 is up-regulated with both doxorubicin and IR, and down-regulated with FX1 treatment.

To begin to understand the interaction between BCL6 inhibition and BCL6 induction by doxorubicin treatment, the intersection between FX1 and doxorubicin was looked at in more detail. In Figure 5.4B a heatmap is shown of the differentially expressed genes in the intersection between FX1 and doxorubicin. Around half of the genes in the intersection between FX1 and doxorubicin were going in opposite directions (24 out of 51), with 20 genes up-regulated in the doxorubicin data-set and down-regulated in the FX1 data-set, and 4 genes downregulated in the doxorubicin data-set and up-regulated in the FX1 data-set. This was 
an interesting finding as it indicated that BCL6 may be involved in the regulation of these differentially expressed genes. Further interpretation of this data is complex, as it is possible that a proportion of these genes in common are related to the potential off-target effects of FX1, and the other half of the genes in common between FX1 and doxorubicin were going in the same direction. 
A.

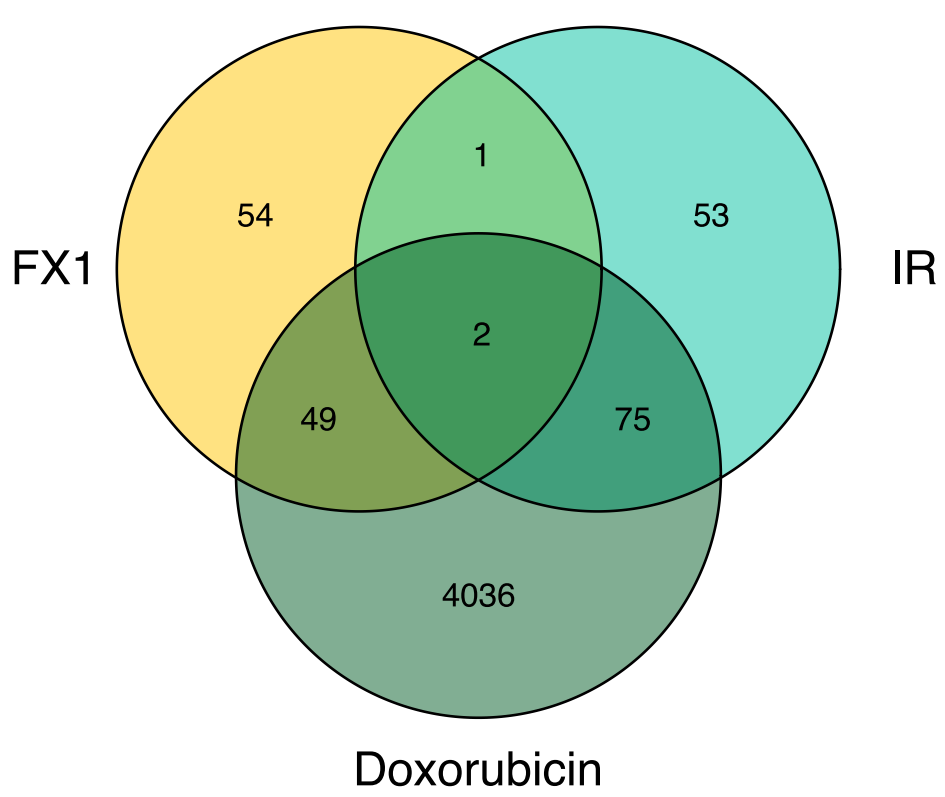

B.

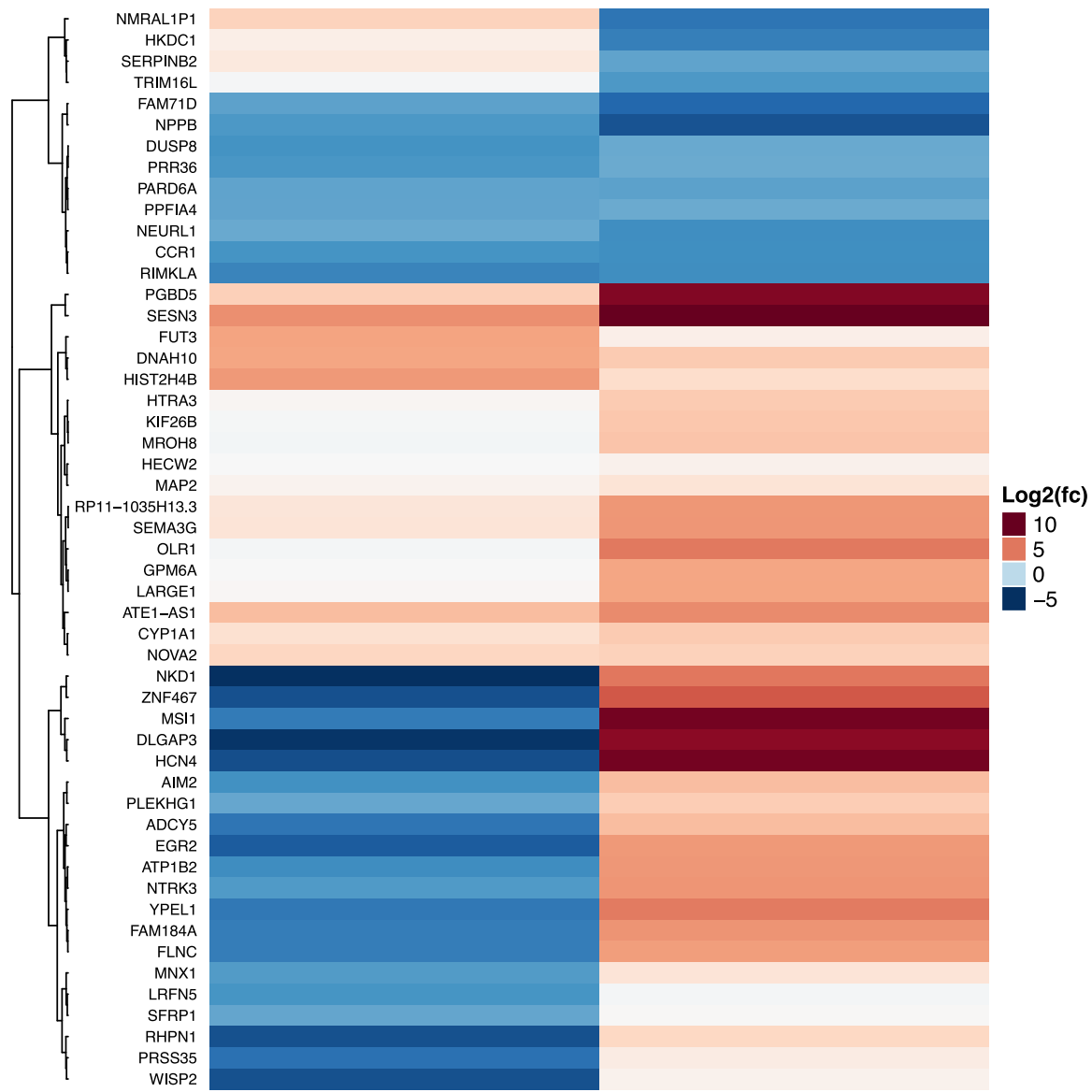

FX1

Doxorubicin

Figure 5.4: Significant differentially expressed genes between data-sets. (A) Venn diagram of significantly differentially expressed genes showing intersects between FX1, doxorubicin and IR data-sets. (B) Heatmap showing $\log 2 \mathrm{fc}$ of the differentially expressed genes in common between the FX1 and doxorubicin datasets. Shades from light red to maroon indicate a $\log 2 \mathrm{fc}$ between $5-10$, light blue to navy blue represents between 0 and -5 , white indicates no expression data. 


\subsubsection{BCL6 Target Genes}

One of the key questions to investigate was: does BCL6 inhibition with FX1 lead to the up-regulation of known BCL6 target genes? If an up-regulation of these genes were seen with FX1 treatment, it would indicate that BCL6 was acting as a transcriptional repressor on these genes in GBM cells, which was not shown by earlier experiments. If no change is seen in these genes, then it backs up what has been observed in previous experiments- that BCL6 is not a transcriptional repressor in GBM cells. A list of BCL6 target genes was created based on the ChIP work completed in this thesis (CCND2, ATR, TARS) and genes that were shown to be de-repressed in lymphoma cells treated with FX1 (CD69, CASP8, CXCR4, CDK1NA, DUSP5)(179) and breast cancer cells (KLF6, PRDM1, SH3PXD2B, HERC5, MED24) (180) and their expression determined.

In Figure 5.5 a heatmap shows z-scores for every replicate of each treatment for each of the BCL6 target genes from the Cardenas et al. (179) and Walker et al. (180) papers, with the BCL6 target genes tested in this thesis (CCND2, ATR, TARS) included in the Cardenas et al. list. The Cardenas et al. genes are a selection of BCL6 target genes previously identified and validated in lymphoma that are de-repressed by FX1. The Walker et al. genes are BCL6 target genes in breast cancer that have been validated by both ChIP and BCL6 inhibition. The z-score describes how many standard deviations away a sample is from the mean expression for a particular gene across all of the samples. In the case of this heatmap, if a sample has high expression of a gene compared to the mean expression of that gene across all samples it will have a positive z-score, represented by shades of the colour red. If a sample has low expression of a gene compared to the mean expression of that gene across all samples it will have a negative $z$-score, represented by shades of the colour blue.

Up-regulation of the BCL6 target genes TARS and KLF6 was seen in the FX1 treated cells. For the remainder of the target genes investigated a slight decrease or no change in transcript was observed in GBM, unlike what has been observed with FX1 treatment in lymphoma cells (179). 
Doxorubicin treatment led to strong up-regulation of most of the BCL6 target genes investigated, with the exception of TARS, KLFG, and PRDM1 which were strongly down-regulated, and ATR, which is slightly up-regulated in one sample and slightly down-regulated in the other two. The strong up-regulation of the remainder of these may not be related to BCL6 expression directly, as doxorubicin tended to increase gene expression overall (Figure 5.3).

In combination with the ChIP data presented in chapter 4 (Figure 4.7), which showed some BCL6 binding to TARS with doxorubicin treatment, this data would suggest that BCL6 acts as a transcriptional repressor on the TARS gene. Though not investigated with ChIP, KLF6 shows a similar pattern to TARS so it seems likely that KLF6 is targeted in the same way by BCL6. The ChIP data presented in Figure 4.7 also showed that BCL6 is not bound to ATR or CCND2 in doxorubicin treated cells, which is further evidence that the changes seen here with doxorubicin treatment are unlikely to be regulated by doxorubicin-induced BCL6.

IR treatment lead to strong up-regulation of SH3PXD2B and a slight up-regulation was also seen with doxorubicin treatment. This was accompanied by a slight downregulation of SH3PXD2B with FX1 treatment, which may indicate that BCL6 is acting as a transcriptional activator in this context. Slight down-regulation of TARS was seen with IR treatment, though this pattern was not as strong as that seen with doxorubicin, and this also reflected the ChIP data presented in Figure 4.9, which showed some BCL6 binding to the TARS gene after irradiation. Overall, few changes were seen in the expression of BCL6 target genes with IR treatment, with most genes slightly decreased in expression, similar to what was observed with the FX1 treated samples. Variability between replicates was seen with the ATR, CD69, and PRDM1 genes, ATR had one strongly and one slightly up-regulated sample, and the third sample slightly down-regulated, CD69 had one sample up-regulated, one sample slightly down-regulated and one sample not changed, and PRDM1 had one sample up-regulated, one sample slightly down-regulated and one sample unchanged. Given that BCL6 was not bound to ATR with BCL6 ChIP, it is unlikely that this variability seen was due to BCL6 regulation of gene expression. 


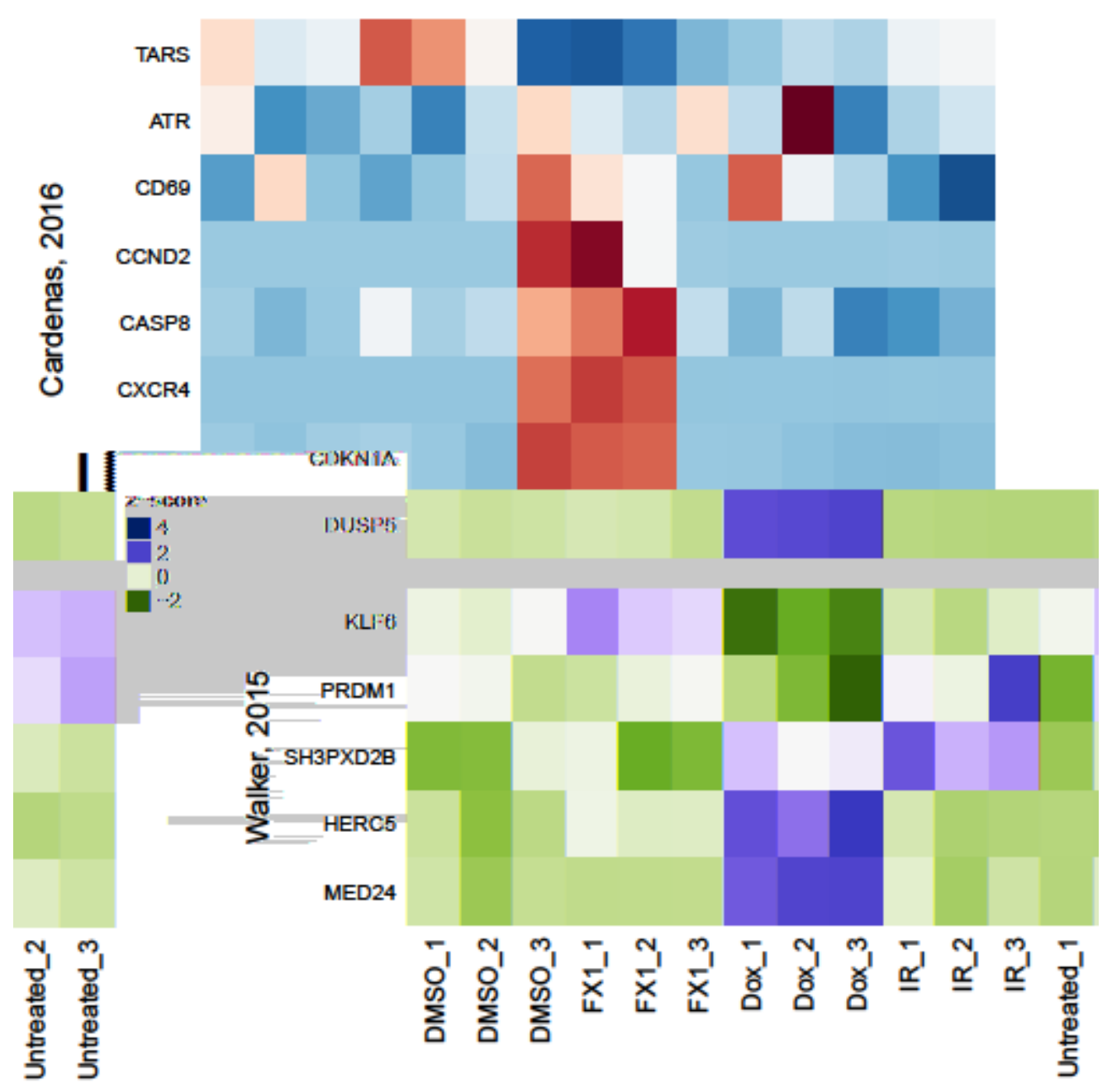

Figure 5.5: Heatmap of BCL6 target gene expression in RNA seq data-sets. BCL6 target genes are listed on the y-axis, with target genes from the Cardenas et al. paper (179) (lymphoma) in the top half and target genes from the Walker et al. paper (180) (breast cancer) in the bottom half. Each set of genes are clustered hierarchically using Ward's method. Each replicate of each treatment group is represented on the $\mathrm{x}$-axis. The values represented are $\mathrm{z}$-scores, based on the standard deviation across all treatments per gene. Shades from light red to maroon indicate a $z$-score between 2-4, and light blue to navy blue represents between 0 and -2 . 


\subsubsection{Gene Set Enrichment Analysis}

Technologies like RNA sequencing give researchers large amounts of information about the gene expression of a cell population under particular conditions. This information usually comprises of large lists of genes, and after DEA is completed, fold change and p-values for these genes. It is extremely difficult to interpret this data in this form, primarily due to the sheer volume of it, and a lack of contextualisation: an increase or decrease in expression of any one gene is unlikely to translate into a meaningful effect on the cells. Gene set enrichment analysis (GSEA) is a tool that was developed by the Broad Institute to address this problem (194). GSEA works by comparing a gene list to others that have been curated and placed in the molecular signatures database (MSigDB), which has over 10,000 genesets that have known functions (195). A list is submitted to GSEA that has been ranked (usually by p-value) to be compared to one (or all) of the databases within the MSigDB and the GSEA software will then calculate an enrichment score (ES), which describes how much of any gene-set that it has been compared against appears at the top or bottom of the list. The ES is normalised based on gene-set size to give a normalised enrichment score (NES). The higher the NES, the more genes at the top of the list are found in that gene-set. Significance of each gene-set is assigned based on the false discovery rate (FDR), which is the estimated probability that a gene-set that is assigned an NES is a false positive, and an FDR $\leq$ 0.25 is the accepted cut-off to reduce false positives without losing true positives. FDR is then used to rank each gene-set within the data-set, and as each gene-set is related to a cellular function or pathway, this gives information about which of these are significantly enriched in the data-set. From here, you now have an idea of the biological consequences of your gene expression data and can start to build hypotheses based on this information.

GSEA is an incredibly powerful tool, and running samples against every gene-set in the MSigDB provides researchers with a large amount of functional information about their data-set. Many of the gene-sets in the MSigDB have functional or gene overlap, which may lead to an overrepresentation of certain gene-sets. This may effectively hide other gene-sets that are significant, as they end up much further down the list of significant gene-sets. As a way of addressing this problem, the Hallmark gene-set collection was developed (215). The Hallmarks collection is a 
collection of 50 gene-sets that were first identified by finding all of the sets in the MSigDB that had genes in common, and experts then manually collated these into biological themes, with a limit of 200 genes per gene-set. The "raw" Hallmark lists were then validated on biological data-sets with known signatures, and from this information the 50 gene-sets were finalised. The Hallmarks collection allows researchers to effectively identify different biological processes represented in their data, without losing information from overrepresentation of a few biological processes.

GSEA was carried out on our RNA seq data using the Hallmarks collection, to get an idea of the biological processes affected by FX1, doxorubicin and IR treatment in GBM cells. Figure $\mathbf{5 . 6}$ shows a dot plot representing the results of the GSEA for each treatment compared to the Hallmarks collection. The cut-offs used for the differentially expressed RNAseq data were padj $<0.05, \log 2 \mathrm{fc}>0.5,<-0.5$. All Hallmark gene-sets shown had a FDR $\leq 0.25$. For this plot, the RNA seq data put into the GSEA was ranked by padj, with the most significant genes at the top of the list, regardless of $\log 2 \mathrm{fc}$. The list shown is ranked by gene ratio, which is the number of genes in the data-set also in the Hallmark gene-set/total number of genes in the Hallmark gene-set. The Hallmarks on the left hand side are ranked in order of gene ratio first based on the FX1, then doxorubicin, then IR, then FX1_Dox data. The size of the dot represents the NES, the larger it is the higher the NES. The colour of the dot represents the FDR, red represents the most significant $(\sim 0)$, pink $(\sim 0.1)$, purple $(\sim 0.15)$ and blue $(\sim 0.2)$ represents the least significant that are still $\leq 0.25$.

In the FX1 column of Figure 5.6, 17 Hallmark gene-sets reached the significance cut-off, and the majority of these were also found with at least one of the other treatments. The 'Myogenesis' and 'Coagulation' Hallmarks were the only ones unique to the FX1 data. The 'Coagulation' gene-set had both a relatively high FDR (0.2) and low NES (1.28) meaning that while it still met the FDR cut-off it is one of the least significant and lowest enriched gene-sets presented. FX1-treated cells had 14 gene-sets in common with the doxorubicin treated cells, 7 gene-sets in common with both doxorubicin and IR, and one gene-set in common with just IR and not doxorubicin ('KRAS Signaling Up'). Many of the gene-sets that were significant 
between just doxorubicin and FX1 were unexpected, particularly the metabolic pathways 'Cholesterol Homeostasis' and 'Fatty Acid Metabolism' and signalling pathways 'Estrogen Response Early' and 'Estrogen Response Late', as it is not clear how these pathways would relate directly to doxorubicin or FX1 treatment, but these may be related to the off target effects of FX1. The 'MTORC1 pathway' hallmark was the most interesting one to be shared between the FX1 and doxorubicin datasets, as the MTOR pathway plays a key role in cell survival and MTOR related proteins frequently have activating mutations in GBM.

Doxorubicin had the most hits for significant Hallmark gene-sets, with 37 in total. Nearly half of these were unique to doxorubicin $(17 / 37,46 \%)$, which further reflected the unique effect that doxorubicin had on these cells. Importantly, the doxorubicin data had many Hallmarks in common with both the FX1 and IR data sets (15 and 12, respectively), which shows that doxorubicin treatment has effects on GBM cells in common with these treatments.

In the IR data-set, 16 Hallmark gene-sets reached the significance cut-off, which is a similar number of Hallmarks seen with FX1 treatment. Also similar to FX1, only two of these are unique to the IR column: 'IL2 STAT5 Signaling' and 'Inflammatory Response'. The majority of the Hallmarks that reached significance in the IR column are in common with doxorubicin (12/15). 'E2F Targets' and 'G2M Checkpoint' are both cell cycle related gene-sets and are both significant in just the doxorubicin and IR data-sets. This makes sense as both treatments cause DNA damage, which results in cell cycle arrest.

The FX1_Dox column has 7 Hallmark gene-sets that reached the significance cutoff, with 4 Hallmarks that were also significant in FX1, Dox, and IR columns. The 'MTORC1 Signaling' and 'Estrogen Response Late' Hallmarks were significant in the FX1_Dox column and in the FX1 and Dox columns. The 'KRAS Signaling Up' Hallmark was significant in the FX1_Dox column and in the FX1 and IR columns.

Figure 5.7 shows a dot plot representing the results of the GSEA for each treatment compared to the Hallmarks collection, this time with the analysis taking 
into account the direction of the fold change values from DEA. For this plot, the RNA seq DEA data put into the GSEA was ranked by padj, with the sign of the $\log 2 \mathrm{fc}$ added (i.e. + if up-regulated, - if down-regulated). The padj and $\log 2 \mathrm{fc}$ cutoffs are the same as in Figure 5.6. The split for up- and down-regulated Hallmarks in this plot was based on NES, if the NES was positive the Hallmark was upregulated, if the NES was negative then the Hallmark was down-regulated, and the negative NES values were then converted to positive numbers. The Hallmarks on the left hand side are ranked in the same order as Figure 5.6 (gene ratio FX1 $>$ Dox $>$ IR). The FX1_Dox intersect is not included in this plot as none of the Hallmarks reached the FDR cut-off once this list was adjusted for direction of change. When the data has been adjusted for direction, almost the same list of Hallmarks gene-sets are significant, with the exception of new Hallmarks 'Notch Signaling', 'Apical Surface', 'Pancreas Beta Cells', and 'KRAS Signaling Dn'. All of these are found in the up-regulated category of the Dox data, and 'KRAS Signaling Dn' was also found to be up-regulated with IR. Only one Hallmark was lost completely- the 'Reactive Oxygen Species Pathway', which was only found in the doxorubicin column of Figure 5.6.

In the FX1 column of Figure 5.713 gene-sets were significant with directional analysis with 8 up-regulated and 5 down-regulated. The FX1 column did not gain any new gene-sets as compared to Figure 5.6 and lost 4 gene-sets: 'Adipogenesis", 'Coagulation', 'Estrogen Response Early', and 'Estrogen Response Late'. 'Coagulation' was the only one that the FX1 data-set didn't have in common with the Doxorubicin data-set with the non-directional analysis. The FX1 column had no unique gene-sets, and had 6 in common with both doxorubicin and IR, and 7 in common with just doxorubicin.

Doxorubicin had the most hits with the directional analysis (45 gene-sets), with 26 up-regulated and 19 down-regulated. The doxorubicin column gained a total of 9 new gene-sets as compared to Figure 5.6: 'Apical Surface', 'Coagulation', 'IL2 STAT5 Signaling', 'Inflammatory Response', 'KRAS Signaling Dn', 'KRAS Signaling Up', 'Myogenesis', 'Notch Signaling', and 'Pancreas Beta Cells', and losing one: 'Reactive Oxygen Species Pathway', as mentioned earlier. The doxorubicin 
column had 22 unique gene-sets, and is the only treatment that had unique genesets.

The IR column had a total of 15 significant gene sets with directional analysis, with 10 up-regulated and 5 down-regulated. The IR column gained 3 new gene-sets as compared to Figure 5.6: 'Coagulation', 'Estrogen Response Late', and 'KRAS Signaling Dn', and lost 4 gene-sets: 'Apoptosis', 'IL2 STAT Signaling', 'Inflammatory Response' and 'Xenobiotic Metabolism'. The IR column had no unique gene-sets, and had 6 in common with both FX1 and doxorubicin, and 9 in common with just Doxorubicin.

The 6 genesets in common between FX1, doxorubicin and IR in the directional analysis were almost entirely the same as those observed with non-directional analysis. With non-directional analysis these gene-sets were (in alphabetical order): 'Apoptosis', 'Epithelial Mesenchymal Transition', 'Glycolysis', 'Hypoxia', 'P53 Pathway', 'TNFA Signaling Via NFKB', and 'Xenobiotic Metabolism'. With directional analysis these gene-sets were: 'Epithelial Mesenchymal Transition', 'Glycolysis', 'Hypoxia', 'KRAS Signaling Up', 'P53 Pathway', and 'TNFA Signaling Via NFKB'.

Adding directionality into the analysis was useful for looking at differences in overall expression of different pathways between treatments. It must be noted that this directional analysis does not necessarily directly translate to activation or deactivation of a pathway, it simply reflects the overall changes in transcript for components of this pathway, which may reflect an activation or deactivation of that pathway. Of the gene-sets that all three treatments had significant hits for, 'TNFA Signaling Via NFKB' was ranked highest by gene ratio. This geneset was upregulated in both the FX1 and doxorubicin data, and down-regulated in the IR data. Tumour necrosis factor $\alpha(\mathrm{TNF} \alpha)$ can be produced in low levels by cancer cells, which can act in an autocrine way to encourage cell survival via the transcription factor nuclear factor kappa-light-chain-enhancer of activated B cells (NFkB) (216). Up-regulation of this pathway could have indicated a survival response by the cells to BCL6 inhibition (FX1 treatment) and doxorubicin treatment, and could have 
indicated that these treatments may have had a similar effect on the cells, despite their different mechanism of action. An alternative explanation may be that this survival response may reflect some off-target effects of FX1 on other kinases being similar to the effects of doxorubicin. Almost all of the gene-sets (with the exception of 'Myogenesis' and 'Xenobiotic Metabolism') that are significant for both FX1 and doxorubicin treated cells were going in the same direction. Ionising radiation appears to be acting differently to FX1 and doxorubicin with regards to the 'TNFA Signaling Via NFKB' gene-set by down-regulating it. However, almost all of the other gene-sets that are significant for both doxorubicin and IR are going in the same direction, with the exception of the 'TNFA Signaling Via NFKB' gene-set and the 'Glycolysis' gene-set.

Of the gene-sets that the FX1 and doxorubicin data-sets had in common, 'MTORC1 Signaling' was the highest ranked by gene ratio, and was down-regulated with both treatments. This geneset refers to the signalling downstream of mammalian target of rapamycin complex 1 (MTORC1). GBM is well known to have mutations in the PI3K/AKT/MTOR pathway, which leads to activation of downstream signalling (28). Signalling downstream of MTORC1 is pro-survival as it leads to amino acid and lipid synthesis as well as autophagy which encourages tumour cell proliferation and growth (217). It seems unusual that GBM cells would down-regulate a pro-survival pathway in response to both BCL6 inhibition and doxorubicin, especially considering both data-sets have up-regulation of other survival pathways. However, with the complex effect of both of these treatments have on cells, it is possible that these cells are prioritising certain pathways over others in the face of BCL6 inhibition or doxorubicin. 

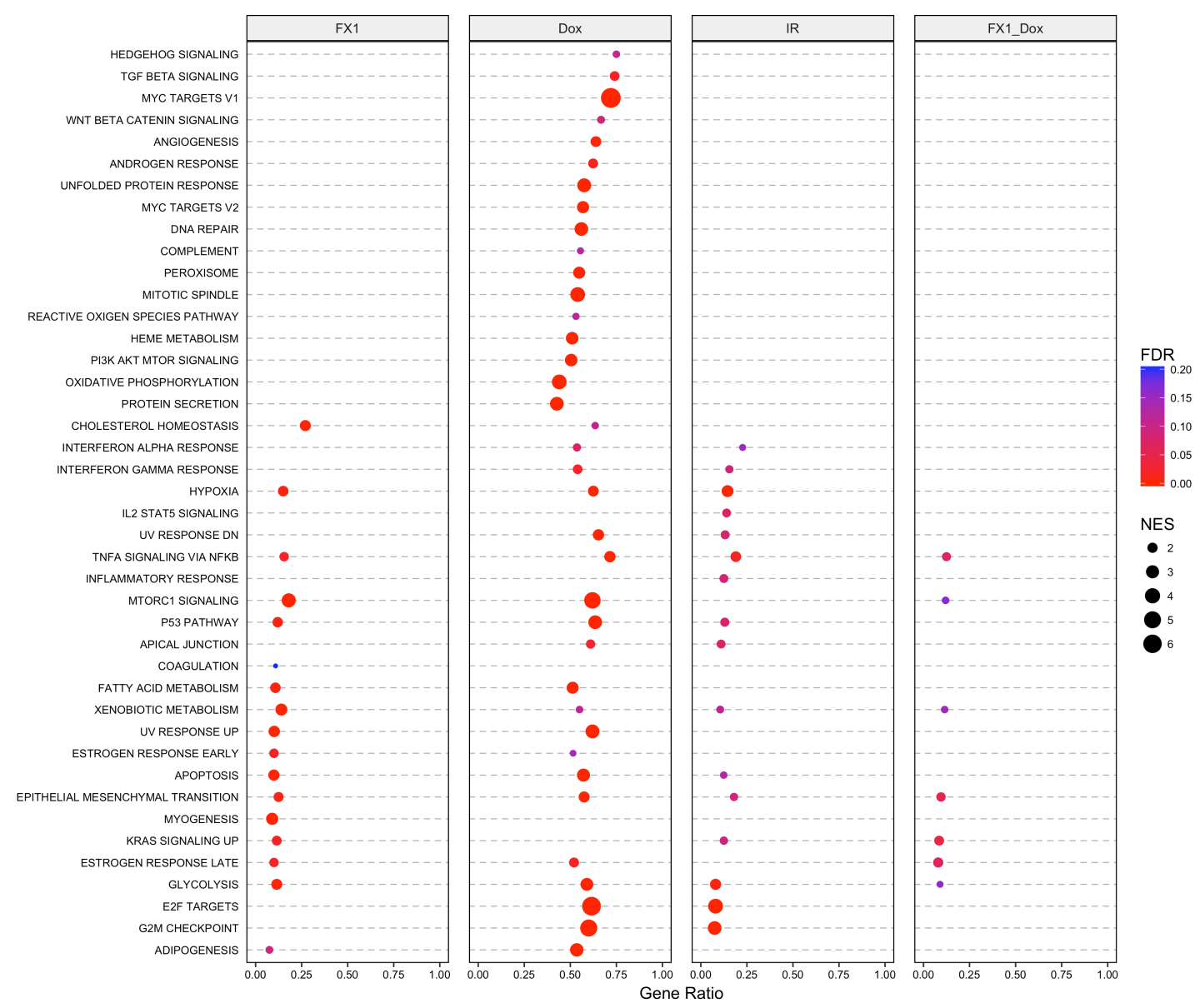

Figure 5.6: Significant GSEA Hallmarks Data-sets. Dotplot showing normalised false discovery rate (FDR), normalised enrichment score (NES), and gene ratio for the Hallmark collection gene-sets in the RNA seq data lists for FX1 v DMSO (FX1), doxorubicin $\mathrm{v}$ untreated (Dox), irradiation $\mathrm{v}$ untreated (IR) and the FX1/doxorubicin intersection (FX1_Dox, see Figure 5.4A). FDR is represented by colour, red representing the most significant $(\sim 0)$, pink $(\sim 0.1)$, purple $(\sim 0.15)$ and blue $(\sim 0.2)$ representing the least significant that still are $\leq 0.25$. NES is represented by size of the dot, the larger the dot the higher the NES. Gene ratio= number of genes in our data-set also in the gene-set/total number of genes in each hallmark gene-set. 


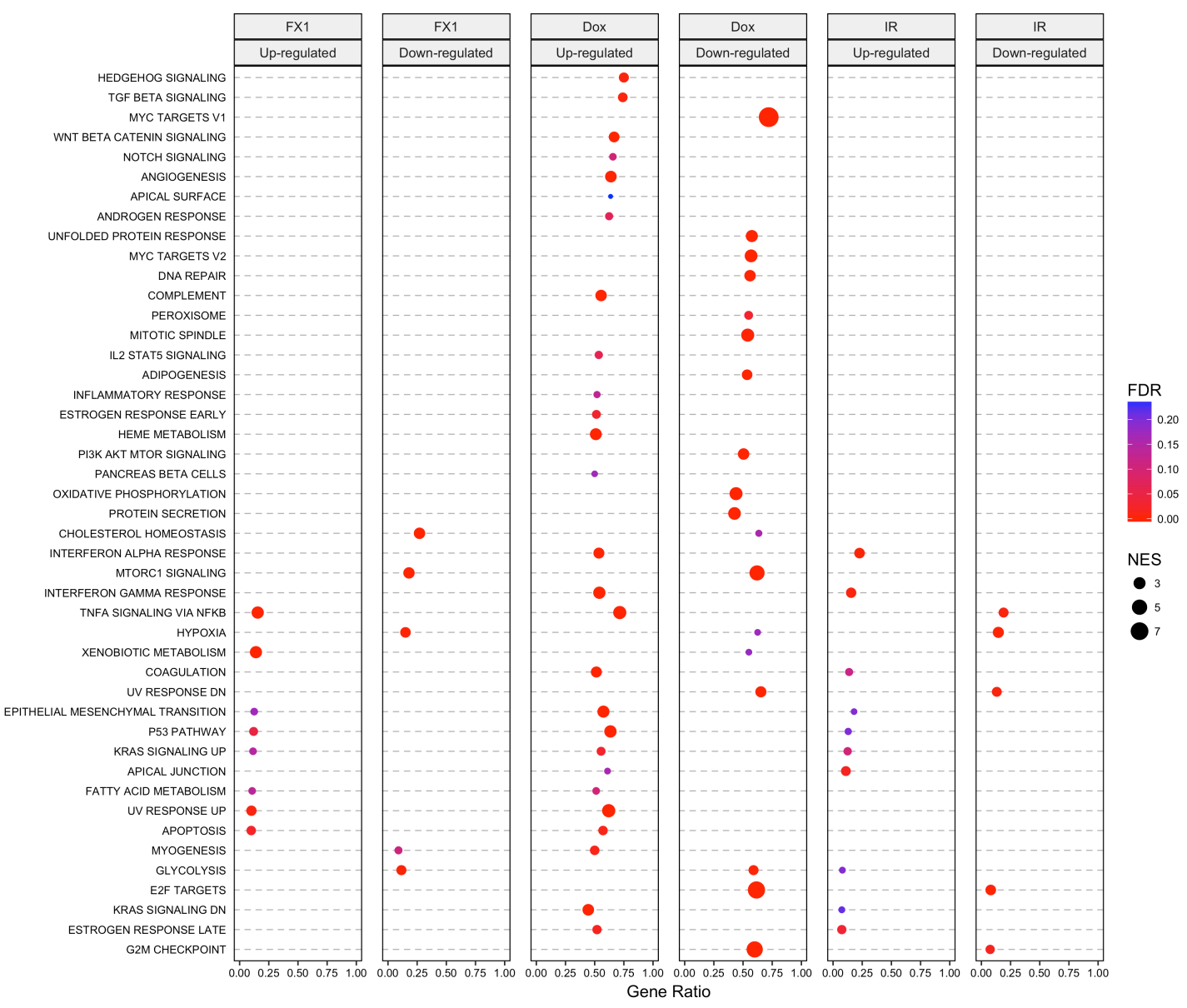

Figure 5.7: Up- and Down-regulated GSEA Hallmarks data-sets. Dotplot showing NES, FDR and gene ratio for the up-regulated and down-regulated (by positive or negative NES) Hallmark collection gene-sets for FX1 v DMSO (FX1), doxorubicin $\mathrm{v}$ untreated (Dox), and irradiation $\mathrm{v}$ untreated (IR). FDR is represented by colour, red representing the most significant $(\sim 0)$, pink $(\sim 0.1)$, purple $(\sim 0.15)$ and blue $(\sim 0.2)$ representing the least significant that still are $\leq 0.25$. NES is represented by size of the dot, the larger the dot the higher the NES. Gene ratio= number of genes in our data-set also in the gene-set/total number of genes in each hallmark gene-set. 


\subsection{Summary}

The RNA sequencing project was designed to look at the effect of FX1, doxorubicin and ionising radiation on LN18 cells. The RNA obtained from these different treatments was good quality. The lowest abundances were from the FX1 treated cells, but these were still well within the parameters required by NZGL. There is no obvious reason why there would be less RNA with FX1 treatment; the cells seemed generally healthy at harvest based on appearance, but this information does suggest that FX1 had a larger effect on the cells than was obviously apparent.

PCA analysis showed that the replicates were similar, and the treatments were different, particularly doxorubicin treatment. DEA showed doxorubicin was very different, with thousands of differentially expressed genes, making comparison to the other treatments difficult, as this had to be taken into account throughout the analysis. The FX1 and IR differential expression data-sets had similar numbers of differentially expressed genes.

BCL6 inhibition with FX1 treatment had little effect on previously described BCL6 target gene expression, and the majority of the BCL6 target genes investigated had some degree of reduced expression. This was further evidence towards the hypothesis that BCL6 has a novel function in the context of GBM and may even be primarily acting as a transcriptional activator.

Doxorubicin had a massive effect on gene expression, which has affected this analysis- a strict $\log 2 \mathrm{fc}$ threshold was used to reduce the number of genes that were dealt with in the initial DEA, but these had to be applied to all data-sets equally to be able to compare between them. This means that the number of genes that passed the threshold in both the FX1 and IR data-sets was low (106 and 131 respectively). Using this low number of genes was not very informative in the GSEA analysis, which is why this $\log 2 \mathrm{fc}$ threshold had to be changed. This does mean that the number of pathway "hits" with GSEA on the doxorubicin treatment data may not be at all relevant to the BCL6 pathway- even those that were in common with FX1 treatment. 
In terms of genes in common, FX1 and IR were almost completely distinct datasets, both had similar numbers in common with doxorubicin, but had only 3 genes in common between them. GSEA showed that FX1 and IR were more similar than it first seemed, with 7 gene-sets in common in the non-directional analysis and 6 in common in the directional analysis. This highlights the importance of gene set enrichment analysis; these two treatments have pathways/processes in common, even if they don't regulate the same specific genes.

When looking at differentially expressed genes, the majority of doxorubicin and FX1 genes in common were going in opposite directions. In particular, when the $\log 2 \mathrm{fc}$ of FX1 was down the $\log 2 \mathrm{fc}$ of doxorubicin was up, and this could be further indication that BCL6 is an activator in the context of doxorubicin.

However, this was contrasted by the directional GSEA results, which showed that almost all of the pathways involved were going in the same direction between FX1 and doxorubicin (except for 'Myogenesis' and 'Xenobiotic Metabolism'). This could mean that these treatments were having similar effects on the cells, and considering doxorubicin also has a strong effect on a whole host of other transcription factors, this may mean effects in common between FX1 and doxorubicin are not necessarily due to BCL6 inhibition. It could also mean that the GSEA analysis has not gone deep enough to really tease out the differences between these two data-sets. It may be difficult to tease out these differences as it is a relatively short list of genes in common between these two data-sets (678 genes), and it is possible that the directional analysis didn't produce significant results because of this short list.

This sequencing was only completed on LN18 cells, which limits the interpretation of the results produced from this analysis. Further investigation of the pathways identified here will need to include many more GBM cell lines as well as primary tissue.

It is important to note that FX1 has had a dramatic effect on the cells transcriptional profile, regardless of whether you look at the gene level or GSEA data. FX1 is a specific inhibitor of BCL6, a protein with very little history in GBM. This result, coupled with the information that FX1 has an $\mathrm{IC}_{50}$ in multiple GBM cell 
lines that is very similar to the $\mathrm{IC}_{50}$ in BCL6-dependent lymphomas, indicates that BCL6 plays a vital role in the survival of GBM cells, even if this role is still not completely clear. 


\section{General Discussion}

\subsection{Introduction}

This thesis set out to evaluate what role BCL6 plays in GBM, and the effect of therapy on BCL6 activity. This work showed that BCL6 was expressed at a basal level in both LN18 and U87-MG cell lines and further expression was induced by DNA damaging treatment in LN18. In addition, the three main co-repressors BCOR, NCOR, and SMRT were expressed at a basal level in both LN18 and U87MG cell lines. This suggested BCL6 would act as a transcriptional repressor, as it does in lymphoma (151). However, while BCL6 did have the machinery to be a transcriptional repressor, it did not act as predicted - it seemed to activate transcription instead. Endogenous BCL6 could bind DNA, and was found bound to positive control loci within exon 1 of the BCL6 gene and the TARS gene in untreated, doxorubicin treated, and irradiated LN18 cells. There was very little binding at the other previously described BCL6 target genes investigated, in both untreated cells, and cells treated with either treatment.

RNA sequencing was carried out to examine the transcriptional profile of GBM cells in response to BCL6 inhibition and DNA damage. This revealed that BCL6 inhibition with the small molecule inhibitor FX1 had a slight down-regulation effect on total transcription, and had little effect on previously described BCL6 target genes, which could be evidence that BCL6 had a novel function in LN18. Doxorubicin treatment caused opposing expression of a subset of FX1 regulated genes, perhaps consistent with increased BCL6 expression, as these genes went up with doxorubcin and down with FX1. GSEA revealed that the pathways in common between the FX1 and doxorubcin data-sets were related to cell survival. While irradiation and doxorubicin both induced DNA damage related pathways, as would be predicted, there was little in common between genes or pathways regulated by IR and FX1 treatments. This was also consistent with the lack of BCL6 up-regulation in LN18 or U87-MG treated with irradiation, despite previous observations of BCL6 up-regulation with IR treatment. 
Overall, the primary aims of this thesis to determine the presence and transcriptional activity of BCL6, to evaluate BCL6 binding of target genes and to look at transcriptional changes in response to BCL6 inhibition all in the context of GBM were evaluated successfully, but there were multiple interesting findings and limitations of the work that will be discussed in more detail.

\subsection{Determine that BCL6 is present and transcriptionally active in GBM cells}

In Chapter 3 of this thesis, the GBM cell lines LN18 and U87-MG were used to investigate the presence of BCL6 in response to therapy and its transcriptional activity. The presence of BCL6 and co-repressors BCOR, NCOR, and SMRT was assessed at transcript and protein level with doxorubicin, irradiation, and FX1 treatments, and showed that BCL6 was present without treatment in both cell lines and was up-regulated with doxorubicin treatment. The co-repressors were also present in both cell lines, untreated and with treatment. The transcriptional activity of BCL6 was assessed by BCL6-specific luciferase assay, which showed that overexpressed BCL6 acted as a repressor, but endogenous BCL6 induced by treatment seemed to act as an activator. BCL6 did not appear to be transcriptionally active in U87-MG.

\subsubsection{BCL6 is transcriptionally active in GBM cells, but it behaves differently than expected}

BCL6 was present in two GBM cell lines LN18 and U87-MG. In these cell lines, there was the necessary machinery, i.e. co-repressors, to be a transcriptional repressor. BCL6 was transcriptionally active in LN18 cells, but did not appear to have transcriptional activity in U87-MG cells. BCL6 was a transcriptional repressor when overexpressed in LN18, but endogenous BCL6 was not a repressor and even appeared to activate transcription with doxorubicin and irradiation treatments. This led to the hypothesis that BCL6 is a transcriptional activator in LN18, which was investigated further by RNA sequencing with BCL6 inhibitor FX1. The RNA sequencing showed that FX1 and doxorubicin treatments had a subset of differentially expressed genes that were down-regulated with FX1 and up-regulated 
with doxorubicin, which indicated that expression of these genes may be activated by BCL6. BCL6 target gene SH3PX2B was up-regulated with IR treatment and down-regulated with doxorubicin, which may indicate that expression of this gene is activated by BCL6 in LN18. These results are further supported by BCL6 being shown to be a transcriptional activator in GBM (187) and in human kidney cells (206).

\subsubsection{Choice of chemotherapy}

Temozolomide is the primary chemotherapeutic used to treat GBM, however it was not used for the experiments conducted for this thesis. This was because TMZ is highly insoluble, and previous experiments conducted in the McConnell lab found that to achieve the effective concentration of TMZ in vitro multiple doses of TMZ were required over a series of days in order to induce BCL6 expression (See Appendix: Fabre, Jones, et al., in revision). The McConnell lab has observed that multiple doses of DMSO, the vehicle required to dissolve TMZ at high concentration, can upregulate BCL6 expression in GBM cells, which can make it difficult to distinguish from TMZ. Doxorubicin is highly soluble and is not dissolved in DMSO, so it was much easier to work with in vitro, as a simpler dosing regimen could be used for these experiments. Doxorubicin reliably induced BCL6 expression in LN18 cells with one dose, which made experiments much shorter than they would have been with TMZ, which allowed more time for optimisation of experiments. Doxorubicin cannot get past the blood brain barrier, which is why it is not currently used to treat GBM. Much work is currently being done to formulate doxorubicin to get it past the blood brain barrier, and we hope to see a trial of this in the future (91-94). Experiments conducted with doxorubicin in this thesis are currently being repeated with TMZ in the McConnell lab.

\subsubsection{Use of cell lines}

One of the major limitations of this work is that after the luciferase assays were completed, the remainder of this study was conducted in one established GBM cell line, LN18. As discussed in the introduction of this thesis, the modelling of GBM is very difficult as it is a highly heterogeneous disease, both within and between 
tumours, and as such, data from one cell line is not going to be reflective of the entire disease. The original plan was to complete all of the experiments in this thesis on 4 cell lines: LN18 and U87-MG from ATCC, and NZG-0906 and NZG-1003, which are primary tumour cell lines developed in the McConnell lab. These lines were chosen based on the initial work done before this project commenced, which looked at the presence of BCL6 with and without therapy in multiple GBM cell lines, which included LN18, U87-MG, NZG-0906, and NZG-1003, as well as in primary tissue (See Appendix: Fabre, Jones, et al., in revision). Alongside the issues with the transcriptional activity of BCL6 in U87-MG, it became clear early on that there was not going to be enough time to complete all of these experiments on all 4 cell lines to the same depth explored in just LN18. This work needs to be repeated in these cell lines and others to establish if the transcriptional activity of BCL6 is the same across many subtypes of GBM.

\subsubsection{Limitations of the Luciferase Assay}

A limitation of the luciferase assay used to assess the transcriptional activity of BCL6 was that the plasmid used for this had no map available and did not express an internal control luciferase, e.g. renilla luciferase, to account for transfection efficiency. This was addressed in multiple ways, where transfection efficiency was assessed in every experiment with GFP transfection, and the plasmid PGL3 was also used, which does not have a BCL6 binding motif. GFP transfection does not necessarily reflect transfection efficiency with other plasmids, but as the BCL6 ${ }_{4}^{-}$ tkLUC plasmid does not express an internal renilla luciferase control, GFP was the best proxy for transfection efficiency that could be used. Transfection with the PGL3 plasmid was not a perfect comparison as it does not have the same promoter as $\mathrm{BCL6}_{4}$-tkLUC, however as no map of this plasmid was available, we were unable to produce an equivalent of this plasmid without the BCL6 binding sites. Also, luciferase values were normalised to cell numbers, which is not a standard analysis of luciferase assay results. This type of analysis was used as it had been observed that different treatments, particularly doxorubicin, had a substantial effect on cell health and may have interfered with the interpretation of the luciferase results. In the future, these experiments need to be repeated with a plasmid where the map is known, so proper controls can be used. 


\subsection{Investigate BCL6 binding to DNA and BCL6 target genes in GBM cells}

Once transcriptional activity had been shown in Chapter 3, Chapter 4 of this thesis investigated the DNA binding ability of BCL6 and potential target genes of BCL6 in GBM. DNA binding ability was assessed by EMSA, and showed that overexpressed BCL6 bound DNA in complex with its co-repressors. Endogenous or induced BCL6 did not appear to bind DNA by EMSA, but BCL6 ChIP did show binding to BCL6 target genes BCL6 Exon 1 and TARS. BCL6 did not appear to bind to the other target genes investigated- CCND2 and ATR.

\subsubsection{Endogenous BCL6 did not bind its consensus sequence in LN18}

When BCL6 was overexpressed in LN18 cells it was able to bind a single consensus sequence, and formed complexes with the known co-repressors BCOR, NCOR, and SMRT, shown by EMSA. Endogenous BCL6, with or without doxorubicin treatment, was unable to bind this same consensus sequence. This may have been due to the probe only containing one copy of the short binding sequence, and these experiments could be repeated with a probe with multiple BCL6 binding motifs. The ChIP assays showed that endogenous BCL6 can bind DNA, as BCL6 was able to bind to BCL6 Exon 1 and TARS, which both contain known BCL6 binding sequences.

\subsubsection{Endogenous BCL6 does act as a transcriptional repressor at the $T A R S$ locus}

BCL6 did not appear to be bound to any of the BCL6 target genes investigated by qChIP in LN18 cells, with the exception of TARS. RNA sequencing revealed that BCL6 is acting as a transcriptional repressor at the TARS locus, as it was strongly upregulated with BCL6 inhibition and down regulated with doxorubicin treatment. TARS encodes for threonyl-tRNA synthetase, a member of the aminoacyl tRNA synthetases which catalyse the aminoacylation of their respective amino acid (218). It was discovered to be a BCL6 target gene by ChIPseq in DLBCL cells (219), and as BCL6 is consistently bound to this locus, it is now used as a positive control in 
BCL6 ChIP assays (Dr. Katerina Hatzi, personal communication). The aminoacyl tRNA synthetases are highly evolutionarily conserved and are crucial for protein synthesis, so it is not clear why BCL6 would inhibit expression of this seemingly crucial enzyme (218).

\subsubsection{Variation in chromatin immunoprecipitation protocols}

When qChIP for BCL6 was performed on LN18 cells treated with doxorubicin in Prof. Ari Melnick's lab at Weill Cornell Medical College, NYC, BCL6 appeared to be bound to the well-known target genes TARS, CCND2, and ATR as well as the control BCL6 Exon 1 sequence. BCL6 was not bound to the negative control sequence BCL6 Intron 9. When these experiments were repeated in Dr. Melanie McConnell's lab at Victoria University of Wellington, New Zealand, the exact same results were not seen. Many modifications were made to these experiments to adapt them to the McConnell lab (sonication with probe sonicator) and to improve the specificity (Dynabeads ${ }^{\circledR}$ and $\beta$-actin control). All of these are likely to have made a difference to the final outcomes of the experiments. The key to successful chromatin IP is the preparation of chromatin, and the sonicator in the McConnell lab was not designed to shear DNA and wore down over time. Another key issue is that that actin can be found in the nucleus, and has been shown to co-localise with RNA pol II, which may have affected the magnitude of binding seen (208-210). To determine if the results were affected by these modifications, these experiments need to be repeated with the sonicator and negative control used initially in the Melnick lab.

\subsubsection{BCL6 ChIP sequencing}

With the exception of TARS, the qChIP experiments did not find known BCL6 target genes in LN18, neither endogenous BCL6 or in response to doxorubicin or radiation. The best way to find the BCL6 target genes in GBM would be to complete sequencing after ChIP was performed. ChIP sequencing (ChIPseq) experiments performed on breast cancer cells revealed that BCL6 target genes were quite different in breast cancer as compared to the well-known targets in lymphoma (180). As a result, it would be fair to hypothesise that BCL6 would have different 
targets in GBM. GBM ChIP sequencing was originally planned to be part of this project, but sonication with the probe sonicator became less efficient after multiple years of use, and could no longer produce fragments of the size required for ChIP sequencing when these experiments were attempted. To be able to give a comprehensive map of BCL6 target genes in GBM in response to doxorubicin and radiation treatment, this work needs to be completed with a sonicator designed for the purpose of DNA shearing.

\subsection{Investigate the effect of BCL6 inhibition on global transcription in GBM cells}

In Chapter 5, to further investigate the effect of BCL6 on transcription in GBM, RNA sequencing was performed on LN18 cells treated with BCL6 inhibitor FX1, doxorubicin and irradiation. Principal component, differential expression and geneset enrichment analyses were performed on the data, which produced a wealth of information about the effect of BCL6 inhibition as well as doxorubicin and irradiation on LN18.

\subsubsection{BCL6 inhibition had no effect on AXL kinase}

A report in 2017 suggested that BCL6 was bound to and activated transcription of receptor tyrosine kinase AXL (187). If AXL is a BCL6 target gene in GBM that is activated by BCL6, it would be expected that inhibition of BCL6 would lead to down-regulation of AXL expression. The AXL transcript was not significantly upor down-regulated in the LN18 differential expression analysis in the FX1 data-set, and when looked at by z-score expression of AXL did not seem to have changed with FX1 treatment. Further investigation is required to confirm the published report that AXL is a BCL6 target gene in GBM. 


\subsubsection{BCL6 inhibition had a profound effect on gene expression}

RNA Sequencing revealed that BCL6 inhibition with FX1 had a significant effect on gene expression in LN18 cells, which was interesting considering FX1 is designed to specifically bind the BTB domain of BCL6. Considering this specificity of FX1, the changes seen are more than would be expected if BCL6 did not play much of a role in GBM, and suggested that BCL6 does play a significant role in GBM. Clonogenicity assays conducted in the McConnell lab add further evidence to this. These were performed on a range of GBM cell lines treated with FX1, and it was found that FX1 treatment dramatically reduced clonogenicity, at $40 \mu \mathrm{M}$ no colonies were seen with all cell lines, and at $25 \mu \mathrm{M}$ a $50-70 \%$ decrease in colony formation was seen with FX1 treatment (See Appendix: Fabre, Jones, et al., in revision).

It was originally planned to create BCL6 somatic cell knockout lines using a zinc finger nuclease (ZFN) for BCL6, which would have been useful for determining the role of BCL6 in GBM. These experiments were attempted in multiple GBM cell lines but a BCL6 knockout cell was not isolated. It was discovered that any cells that had a successful BCL6 knockout had died, as successful BCL6 knockout was only detected in the dead cell fraction by PCR (Matthew Rowe, personal communication). The BCL6 knockout was detected in HEK293 cells, as the knockout was found in the live cell fraction by PCR, which showed that the construct was working. A ZFN was then obstained for ADP-dependent glucokinase (ADP-GPK) and this gene was successfully knocked out in the GBM cells previously used for the BCL6 knockout. The knockout was found in the live cell fraction by PCR, which showed that the transfection itself was not harming the cells. Overall, these experiments provided further evidence that BCL6 played an important role in these cells, since the loss of BCL6 seemed to cause these cells to die.

\subsubsection{Limited relationship between BCL6 inhibition and ionising radiation}

Ionising radiation led to a 3-fold increase in BCL6 reporter luciferase activity, which indicated that irradiation had led to a large increase in transcriptional activity of BCL6 in LN18. This was a much more dramatic increase in transcriptional activity as compared to doxorubicin. This finding provided strong evidence that BCL6 
induced by irradiation of LN18 was acting as a transcriptional activator. Experiments were continued with irradiation after this result, since there seemed to be something specific to irradiation that activated BCL6.

Differential expression analysis on the RNA sequencing data showed that the FX1 and IR data-sets had only three differentially expressed genes in common. Irradiation did seem to up-regulate BCL6 target gene SH3PXD2B alongside a slight down-regulation of the same gene with FX1 treatment, which may indicate that this is a BCL6 target gene in GBM, and this needs to be validated by ChIP and qPCR. When the GSEA was performed against the Hallmarks collection with the differentially expressed data, FX1 and IR did have some gene-sets in common, but these were usually also in common with doxorubicin, which may indicate that these gene-sets are more fundamental responses to stress caused by treatment than directly related to the treatments themselves. This continued a pattern of irradiation having a different effect on GBM cells compared to the other treatments. Experiments previously completed in the McConnell lab indicated that IR did cause up-regulation of BCL6 protein in a range of GBM cell lines including LN18 and U87-MG (See Appendix: Fabre, Jones, et al., in revision), but this was not seen in the experiments presented in this thesis for both LN18 and U87-MG cell lines, although BCL6 up-regulation was seen with doxorubicin, which had been observed before. Following the result that irradiation led to increased transcription via BCL6, it was interesting that the effect of BCL6 inhibition (FX1 treatment) on LN18 was almost completely distinct from irradiation. This finding needs to be investigated further, possibly by using the other databases in the MSigDB which cover a range of different things, including chromosomal position and motifs, as these may give further insight into commonalities between these two data-sets.

RNA sequencing may have revealed new potential target genes for BCL6 in GBM, and these need to be validated and investigated further in multiple cell lines. It also revealed pathways that could be targeted in combination with BCL6 inhibition in GBM and would be interesting to investigate in both in vitro and in vivo models. 


\subsection{Future Directions}

In the future, experiments need to be done to determine the efficacy of FX1 on in vivo models of glioblastoma, ideally ones that accurately reflect human disease, alongside standard chemotherapy and radiation treatment. It has yet to be determined whether certain types of glioblastoma or possibly glioma (i.e. MGMT +/- or IDH1 +/-) are more likely to respond to FX1 treatment, so further investigation into the transcriptional activity of BCL6 in different GBM tumours or cell lines will help inform if FX1 treatment should be favourable for certain patients.

\subsection{Summary}

BCL6 is a highly characterised driver of lymphoma and based on its involvement in preventing cell death in response to DNA damage, the McConnell lab decided to investigate if it had any role to play in therapy resistance of GBM. This thesis has expanded on the initial results that indicated BCL6 was up-regulated in response to therapy. The results presented in this thesis showed that BCL6 does seem to play an important role in GBM cells, though that role is not yet completely clear. 


\section{$7 \quad$ References}

1. Kettenmann H, Ransom B. The Concept of Neuroglia: A Historical Perspective. In: Neuroglia. Oxford University Press; 2004.

2. Butt AM. Structure and Function of Oligodenrocytes. In: Neuroglia. Oxford University Press; 2004. p. 36-47.

3. Nishiyama A, Komvita M, Suzuki R, Zhu X. Polydendrocytes (NG2 cells): multifunctional cells with lineage plasticity. Nat Rev Neurosci. 2009;9(22).

4. Verkhratsky A, Zorec R, Parpura V. Stratification of astrocytes in healthy and diseased brain. Brain Pathol. 2017 Sep;27(5):629-44.

5. Ge W-P, Miyawaki A, Gage FH, Jan YN, Jan LY. Local generation of glia is a major astrocyte source in postnatal cortex. Nature. 2012 Mar 28;484(7394):376-80.

6. Network TCGAR. Comprehensive, Integrative Genomic Analysis of Diffuse Lower-Grade Gliomas. N Engl J Med. 2015 Jun 25;372(26):2481-98.

7. Louis DN, Perry A, Reifenberger G, von Deimling A, Figarella-Branger D, Cavenee WK, et al. The 2016 World Health Organization Classification of Tumors of the Central Nervous System: a summary. Acta Neuropathol. 2016 Jun 9;131(6):803-220.

8. Tandon A, Schiff D. Therapeutic Decision Making in Patients with Newly Diagnosed Low Grade Glioma. Curr Treat Options Oncol. 2014 Dec 20;15(4):529-38.

9. Oberheim Bush NA, Chang S. Treatment Strategies for Low-Grade Glioma in Adults. J Oncol Pract. 2016 Dec;12(12):1235-41.

10. Buckner JC, Shaw EG, Pugh SL, Chakravarti A, Gilbert MR, Barger GR, et al. Radiation plus Procarbazine, CCNU, and Vincristine in Low-Grade Glioma. N Engl J Med. 2016 Apr 7;374(14):1344-55.

11. Stoddard BL, Dean A, Koshland DE. Structure of isocitrate dehydrogenase with isocitrate, nicotinamide adenine dinucleotide phosphate, and calcium at 2.5-A resolution: a pseudo-Michaelis ternary complex. Biochemistry. 1993 Sep 14;32(36):9310-6.

12. Al-Khallaf $H$. Isocitrate dehydrogenases in physiology and cancer: biochemical and molecular insight. Cell Biosci. 2017 Dec 3;7(1):37.

13. Molenaar RJ, Radivoyevitch T, Maciejewski JP, van Noorden CJF, Bleeker 
FE. The driver and passenger effects of isocitrate dehydrogenase 1 and 2 mutations in oncogenesis and survival prolongation. Biochim Biophys Acta Rev Cancer. 2014 Dec;1846(2):326-41.

14. Leonardi R, Subramanian C, Jackowski S, Rock CO. Cancer-associated isocitrate dehydrogenase mutations inactivate NADPH-dependent reductive carboxylation. J Biol Chem. 2012 Apr 27;287(18):14615-20.

15. Xu W, Yang H, Liu Y, Yang Y, Wang P, Kim S-H, et al. Oncometabolite 2Hydroxyglutarate Is a Competitive Inhibitor of $\alpha$-Ketoglutarate-Dependent Dioxygenases. Cancer Cell. 2011;19(1):17-30.

16. Noushmehr H, Weisenberger DJ, Diefes K, Phillips HS, Pujara K, Berman $\mathrm{BP}$, et al. Identification of a $\mathrm{CpG}$ island methylator phenotype that defines a distinct subgroup of glioma. Cancer Cell. 2010 May 18;17(5):510-22.

17. Turcan S, Rohle D, Goenka A, Walsh LA, Fang F, Yilmaz E, et al. IDH1 mutation is sufficient to establish the glioma hypermethylator phenotype. Nature. 2012 Feb 15;483(7390):479-83.

18. Ohgaki H, Kleihues P. The Definition of Primary and Secondary Glioblastoma. Clin Cancer Res. 2013;19(4).

19. Ohgaki H, Kleihues P. Genetic Pathways to Primary and Secondary Glioblastoma. Am J Pathol. 2007;170(5):1445-53.

20. Stupp R, Hegi ME, Mason WP, van den Bent MJ, Taphoorn MJ, Janzer RC, et al. Effects of radiotherapy with concomitant and adjuvant temozolomide versus radiotherapy alone on survival in glioblastoma in a randomised phase III study: 5-year analysis of the EORTC-NCIC trial. Lancet Oncol. 2009;10(5):459-66.

21. Balss J, Meyer J, Mueller W, Korshunov A, Hartmann C, von Deimling A. Analysis of the IDH1 codon 132 mutation in brain tumors. Acta Neuropathol. 2008 Dec 5;116(6):597-602.

22. Ceccarelli M, Barthel FP, Malta TM, Sabedot TS, Salama SR, Murray BA, et al. Molecular Profiling Reveals Biologically Discrete Subsets and Pathways of Progression in Diffuse Glioma. Cell. 2016;164(3):550-63.

23. Yang P, Zhang W, Wang Y, Peng X, Chen B, Qiu X, et al. IDH mutation and MGMT promoter methylation in glioblastoma: results of a prospective registry. Oncotarget. 2015 Dec 1;6(38):40896-906.

24. Fontana L, Tabano S, Bonaparte E, Marfia G, Pesenti C, Falcone R, et al. 
MGMT- Methylated Alleles Are Distributed Heterogeneously Within Glioma Samples Irrespective of IDH Status and Chromosome 10q Deletion. J Neuropathol Exp Neurol. 2016 Aug;75(8):791-800.

25. Patel AP, Tirosh I, Trombetta JJ, Shalek AK, Gillespie SM, Wakimoto H, et al. Single-cell RNA-seq highlights intratumoral heterogeneity in primary glioblastoma. Science (80- ). 2014;344(6190).

26. Verhaak RGW, Hoadley KA, Purdom E, Wang V, Qi Y, Wilkerson MD, et al. Integrated Genomic Analysis Identifies Clinically Relevant Subtypes of Glioblastoma Characterized by Abnormalities in PDGFRA, IDH1, EGFR, and NF1. Cancer Cell. 2010;17(1):98-110.

27. McLendon R, Friedman A, Bigner D, Van Meir EG, Brat DJ, M. Mastrogianakis $G$, et al. Comprehensive genomic characterization defines human glioblastoma genes and core pathways. Nature. 2008 Oct 23;455(7216):1061-8.

28. Brennan R.G.W. Verhaak, A. McKenna, B. Campos, H. Noushmehr, S. R. Salama, S. Zheng, D. Chakravarty, J. Z. Sanborn, S. H. Berman, R. Beroukhim, B. Bernard, C-J. Wu, G. Genovese, I. Shmulevich, J. BarnholtzSloan, L. Zou, R. Vegesna, S. A. Shukla, G. Ciri CW. The Somatic Genomic Landscape of Glioblastoma. Cell. 10 October. 2013;155(2):462-477.

29. Wiestler B, Capper D, Hovestadt V, Sill M, Jones DTW, Hartmann C, et al. Assessing CpG island methylator phenotype, 1p/19q codeletion, and MGMT promoter methylation from epigenome-wide data in the biomarker cohort of the NOA-04 trial. Neuro Oncol. 2014 Dec 1;16(12):1630-8.

30. Diserens AC, de Tribolet N, Martin-Achard A, Gaide AC, Schnegg JF, Carrel S. Characterization of an established human malignant glioma cell line: LN18. Acta Neuropathol. 1981 Mar;53(1):21-8.

31. PONTÉN J, MACINTYRE EH. LONG TERM CULTURE OF NORMAL AND NEOPLASTIC HUMAN GLIA. Acta Pathol Microbiol Scand. 1968 Aug 18;74(4):465-86.

32. Ishii N, Maier D, Merlo A, Tada M, Sawamura Y, Diserens A-C, et al. Frequent Co-Alterations of TP53, p16/CDKN2A, p14 ARF , PTEN Tumor Suppressor Genes in Human Glioma Cell Lines. Brain Pathol. 1999 Jul;9(3):469-79.

33. Lee SY. Temozolomide resistance in glioblastoma multiforme. Genes Dis. 
2016;3(3):198-210.

34. Allen M, Bjerke M, Edlund H, Nelander S, Westermark B. Origin of the U87MG glioma cell line: Good news and bad news. Sci Transl Med. 2016;8(354).

35. Jones TR, Bigner SH, Schold SC, Eng LF, Bigner DD, Bigner DD. Anaplastic human gliomas grown in athymic mice. Morphology and glial fibrillary acidic protein expression. Am J Pathol. 1981 Dec;105(3):316-27.

36. Miller TE, Liau BB, Wallace LC, Morton AR, Xie Q, Dixit D, et al. Transcription elongation factors represent in vivo cancer dependencies in glioblastoma. Nature. 2017 Jul 5;

37. Bao S, Wu Q, Mclendon RE, Hao Y, Shi Q, Hjelmeland AB, et al. Glioma stem cells promote radioresistance by preferential activation of the DNA damage response. Nature. 2006;444:756-60.

38. Eramo A, Ricci-Vitiani L, Zeuner A, Pallini R, Lotti F, Sette G, et al. Chemotherapy resistance of glioblastoma stem cells. Cell Death Differ. 2006 Jul 3;13(7):1238-41.

39. Suvà ML, Rheinbay E, Gillespie SM, Patel AP, Wakimoto H, Rabkin SD, et al. Reconstructing and Reprogramming the Tumor-Propagating Potential of Glioblastoma Stem-like Cells. Cell. 2014;157(3):580-94.

40. Roos A, Ding Z, Loftus JC, Tran NL. Molecular and Microenvironmental Determinants of Glioma Stem-Like Cell Survival and Invasion. Front Oncol. 2017 Jun 16;7:120.

41. Huang Q, Zhang Q-B, Dong J, Wu Y-Y, Shen Y-T, Zhao Y-D, et al. Glioma stem cells are more aggressive in recurrent tumors with malignant progression than in the primary tumor, and both can be maintained longterm in vitro. BMC Cancer. 2008 Dec 22;8(1):304.

42. Cheng L, Wu Q, Guryanova OA, Huang Z, Huang Q, Rich JN, et al. Elevated invasive potential of glioblastoma stem cells. Vol. 406, Biochemical and Biophysical Research Communications. 2011.

43. Lee J, Kotliarova S, Kotliarov Y, Li A, Su Q, Donin NM, et al. Tumor stem cells derived from glioblastomas cultured in bFGF and EGF more closely mirror the phenotype and genotype of primary tumors than do serumcultured cell lines. Cancer Cell. 2006;9(5):391-403.

44. William D, Mullins CS, Schneider B, Orthmann A, Lamp N, Krohn M, et al. 
Optimized creation of glioblastoma patient derived xenografts for use in preclinical studies. J Transl Med. 2017 Feb 9;15(1):27.

45. Carlson BL, Pokorny JL, Schroeder MA, Sarkaria JN. Establishment, maintenance and in vitro and in vivo applications of primary human glioblastoma multiforme (GBM) xenograft models for translational biology studies and drug discovery. Curr Protoc Pharmacol. 2011 Mar;Chapter 14(14):Unit 14.16.

46. Joo KM, Kim J, Jin J, Kim M, Seol HJ, Muradov J, et al. Patient-Specific Orthotopic Glioblastoma Xenograft Models Recapitulate the Histopathology and Biology of Human Glioblastomas In Situ. Cell Rep. 2013;3(1):260-73.

47. Seligman AM, Shear MJ, Alexander L. Studies in Carcinogenesis: VIII. Experimental Production of Brain Tumors in Mice with Methylcholanthrene. Am J Cancer. 1939;37(3).

48. Ausman JI, Shapiro WR, Rall DP. Studies on the Chemotherapy of Experimental Brain Tumors: Development of an Experimental Model. Cancer Res. 1970;30(9).

49. Akbasak A, Oldfield EH, Saris SC. Expression and modulation of major histocompatibility antigens on murine primary brain tumor in vitro. J Neurosurg. 1991 Dec;75(6):922-9.

50. Herrlinger U, Kramm CM, Johnston KM, Louis DN, Finkelstein D, Reznikoff G, et al. Vaccination for experimental gliomas using GM-CSFtransduced glioma cells. Cancer Gene Ther. 1997;4(6):345-52.

51. Plautz GE, Touhalisky JE, Shu S. Treatment of Murine Gliomas by Adoptive Transfer of ex Vivo Activated Tumor-Draining Lymph Node Cells. Cell Immunol. 1997 Jun;178(2):101-7.

52. Szatmari T, Lumniczky K, Desaknai S, Trajcevski S, Hidvegi EJ, Hamada H, et al. Detailed characterization of the mouse glioma 261 tumor model for experimental glioblastoma therapy. Cancer Sci. 2006 Jun;97(6):546-53.

53. Brinster RL, Chen HY, Messing A, van Dyke T, Levine AJ, Palmiter RD. Transgenic mice harboring SV40 t-antigen genes develop characteristic brain tumors. Cell. 1984 Jun;37(2):367-79.

54. de Vries NA, Beijnen JH, van Tellingen O. High-grade glioma mouse models and their applicability for preclinical testing. Cancer Treat Rev. 2009 Dec;35(8):714-23. 
55. Huszthy PC, Daphu I, Niclou SP, Stieber D, Nigro JM, Sakariassen PØ, et al. In vivo models of primary brain tumors: pitfalls and perspectives. Neuro Oncol. 2012 Aug;14(8):979-93.

56. Stylli SS, Luwor RB, Ware TMB, Tan F, Kaye AH. Mouse models of glioma. J Clin Neurosci. 2015 Apr;22(4):619-26.

57. Weissenberger J, Steinbach JP, Malin G, Spada S, Rülicke T, Aguzzi A. Development and malignant progression of astrocytomas in GFAP-v-src transgenic mice. Oncogene. 1997 Dec 18;14(17):2005-13.

58. Hambardzumyan D, Amankulor NM, Helmy KY, Becher OJ, Holland EC. Modeling Adult Gliomas Using RCAS/t-va Technology. Transl Oncol. 2009 May;2(2):89-95.

59. Alcantara Llaguno S, Chen J, Kwon C-H, Jackson EL, Li Y, Burns DK, et al. Malignant Astrocytomas Originate from Neural Stem/Progenitor Cells in a Somatic Tumor Suppressor Mouse Model. Cancer Cell. 2009;15(1):45-56.

60. Zuckermann M, Hovestadt V, Knobbe-Thomsen CB, Zapatka M, Northcott PA, Schramm K, et al. Somatic CRISPR/Cas9-mediated tumour suppressor disruption enables versatile brain tumour modelling. Nat Commun. 2015 Jun 11;6:7391.

61. Chen J, Li Y, Yu T-S, McKay RM, Burns DK, Kernie SG, et al. A restricted cell population propagates glioblastoma growth after chemotherapy. Nature. 2012 Aug 23;488(7412):522-6.

62. Zhu Y, Guignard F, Zhao D, Liu L, Burns DK, Mason RP, et al. Early inactivation of p53 tumor suppressor gene cooperating with NF1 loss induces malignant astrocytoma. Cancer Cell. 2005 Aug;8(2):119-30.

63. Matsuoka S, Huang M, Elledge SJ. Linkage of ATM to Cell Cycle Regulation by the Chk2 Protein Kinase. Science (80- ). 1998;282(5395).

64. Matsuoka S, Ballif BA, Smogorzewska A, McDonald ER, Hurov KE, Luo J, et al. ATM and ATR Substrate Analysis Reveals Extensive Protein Networks Responsive to DNA Damage. Science (80- ). 2007;316(5828).

65. Mattiroli F, Vissers JHA, van Dijk WJ, Ikpa P, Citterio E, Vermeulen W, et al. RNF168 Ubiquitinates K13-15 on H2A/H2AX to Drive DNA Damage Signaling. Cell. 2012 Sep;150(6):1182-95.

66. Maréchal A, Zou L. DNA damage sensing by the ATM and ATR kinases. Cold Spring Harb Perspect Biol. 2013 Sep 1;5(9):a012716. 
67. Olive KP, Tuveson DA, Ruhe ZC, Yin B, Willis NA, Bronson RT, et al. Mutant p53 Gain of Function in Two Mouse Models of Li-Fraumeni Syndrome. Cell. 2004;119(6):847-60.

68. Lang GA, Iwakuma T, Suh Y-A, Liu G, Rao VA, Parant JM, et al. Gain of Function of a p53 Hot Spot Mutation in a Mouse Model of Li-Fraumeni Syndrome. Cell. 2004;119(6):861-72.

69. Liu DP, Song H, Xu Y. A common gain of function of p53 cancer mutants in inducing genetic instability. Oncogene. 2010 Feb 18;29(7):949-56.

70. Erasimus H, Gobin M, Niclou S, Van Dyck E. DNA repair mechanisms and their clinical impact in glioblastoma. Mutat Res Mutat Res. 2016;769:19-35.

71. Hegde ML, Hazra TK, Mitra S. Early steps in the DNA base excision/singlestrand interruption repair pathway in mammalian cells. Cell Res. 2008 Jan;18(1):27-47.

72. Radhakrishnan SK, Jette N, Lees-Miller SP. Non-homologous end joining: Emerging themes and unanswered questions. DNA Repair (Amst). 2014;17:2-8.

73. Wang C, Lees-Miller SP. Detection and Repair of Ionizing RadiationInduced DNA Double Strand Breaks: New Developments in Nonhomologous End Joining. Int J Radiat Oncol. 2013 Jul;86(3):440-9.

74. Sartori AA, Lukas C, Coates J, Mistrik M, Fu S, Bartek J, et al. Human CtIP promotes DNA end resection. Nature. 2007 Nov 22;450(7169):509-14.

75. Limbo O, Chahwan C, Yamada Y, de Bruin RAM, Wittenberg C, Russell P. Ctp1 Is a Cell-Cycle-Regulated Protein that Functions with Mre11 Complex to Control Double-Strand Break Repair by Homologous Recombination. Mol Cell. 2007 Oct 12;28(1):134-46.

76. de Jager M, van Noort J, van Gent DC, Dekker C, Kanaar R, Wyman C. Human Rad50/Mre11 is a flexible complex that can tether DNA ends. Mol Cell. 2001 Nov;8(5):1129-35.

77. Jasin M, Rothstein R. Repair of strand breaks by homologous recombination. Cold Spring Harb Perspect Biol. 2013 Nov 1;5(11):a012740.

78. Roos WP, Bernd K. DNA damage-induced cell death: From specific DNA lesions to the DNA damage response and apoptosis. Cancer Lett. 2013;332(2):237-48.

79. Matt S, Hofmann TG. The DNA damage-induced cell death response: a 
roadmap to kill cancer cells. Cell Mol Life Sci. 2016;73(15):2829-50.

80. Riedl SJ, Salvesen GS. The apoptosome: signalling platform of cell death. Nat Rev Mol Cell Biol. 2007 May 21;8(5):405-13.

81. Youle RJ, Strasser A. The BCL-2 protein family: opposing activities that mediate cell death. Nat Rev Mol Cell Biol. 2008 Jan;9(1):47-59.

82. Onoyama Y, Abe M, Yabumoto E, Sakamoto T, Nishidai T. Radiation therapy in the treatment of glioblastoma. AJR Am J Roentgenol. 1976 Mar;126(3):481-92.

83. Walker MD, Green SB, Byar DP, Alexander E, Batzdorf U, Brooks WH, et al. Randomized Comparisons of Radiotherapy and Nitrosoureas for the Treatment of Malignant Glioma after Surgery. N Engl J Med. 1980 Dec 4;303(23):1323-9.

84. Oda E, Ohki R, Murasawa H, Nemoto J, Shibue T, Yamashita T, et al. Noxa, a BH3-Only Member of the Bcl-2 Family and Candidate Mediator of p53Induced Apoptosis. Science (80- ). 2000;288(5468).

85. Stupp R, Mason WP, van den Bent MJ, Weller M, Fisher B, Taphoorn MJB, et al. Radiotherapy plus Concomitant and Adjuvant Temozolomide for Glioblastoma. N Engl J Med. 2005 Mar 10;352(10):987-96.

86. Mojas N, Lopes M, Jiricny J. Mismatch repair-dependent processing of methylation damage gives rise to persistent single-stranded gaps in newly replicated DNA. Genes Dev. 2007 Dec 15;21(24):3342-55.

87. Roos WP, Batista LFZ, Naumann SC, Wick W, Weller M, Menck CFM, et al. Apoptosis in malignant glioma cells triggered by the temozolomide-induced DNA lesion O6-methylguanine. Oncogene. 2007 Jan 11;26(2):186-97.

88. Fu D, Calvo JA, Samson LD. Balancing repair and tolerance of DNA damage caused by alkylating agents. Nat Rev Cancer. 2012 Jan 12;12(2):104.

89. Molenaar RJ, Verbaan D, Lamba S, Zanon C, Jeuken JWM, Boots-Sprenger SHE, et al. The combination of IDH1 mutations and MGMT methylation status predicts survival in glioblastoma better than either IDH1 or MGMT alone. Neuro Oncol. 2014 Sep 1;16(9):1263-73.

90. Lyseng-Williamson KA, Duggan ST, Keating GM. Pegylated Liposomal Doxorubicin: A Guide to Its Use in Various Malignancies. BioDrugs. 2013 Oct 10;27(5):533-40.

91. Gaillard PJ, Appeldoorn CCM, Dorland R, van Kregten J, Manca F, Vugts 
DJ, et al. Pharmacokinetics, Brain Delivery, and Efficacy in Brain TumorBearing Mice of Glutathione Pegylated Liposomal Doxorubicin (2B3-101). Velasco G, editor. PLoS One. 2014 Jan 8;9(1):e82331.

92. Aryal M, Vykhodtseva N, Zhang Y-Z, McDannold N. Multiple sessions of liposomal doxorubicin delivery via focused ultrasound mediated blood-brain barrier disruption: A safety study. J Control Release. 2015 Apr;204:60-9.

93. Li J, Cai P, Shalviri A, Henderson JT, He C, Foltz WD, et al. A Multifunctional Polymeric Nanotheranostic System Delivers Doxorubicin and Imaging Agents across the Blood-Brain Barrier Targeting Brain Metastases of Breast Cancer. ACS Nano. 2014 Oct 28;8(10):9925-40.

94. Wen L, Tan Y, Dai S, Zhu Y, Meng T, Yang X, et al. VEGF-mediated tight junctions pathological fenestration enhances doxorubicin-loaded glycolipidlike nanoparticles traversing BBB for glioblastoma-targeting therapy. Drug Deliv. 2017 Jan 28;24(1):1843-55.

95. Yang F, Teves SS, Kemp CJ, Henikoff S. Doxorubicin, DNA torsion, and chromatin dynamics. Biochim Biophys Acta. 2014 Jan;1845(1):84-9.

96. Pommier Y, Leo E, Zhang H, Marchand C. DNA Topoisomerases and Their Poisoning by Anticancer and Antibacterial Drugs. Chem Biol. 2010 May 28;17(5):421-33.

97. Tacar O, Sriamornsak P, Dass CR. Doxorubicin: an update on anticancer molecular action, toxicity and novel drug delivery systems. J Pharm Pharmacol. 2013 Feb;65(2):157-70.

98. Hanahan D, Weinberg RA. Hallmarks of Cancer: The Next Generation. Cell. 2011;144(5):646-74.

99. Hunter C, Smith R, Cahill DP, Stephens P, Stevens C, Teague J, et al. A Hypermutation Phenotype and Somatic MSH6 Mutations in Recurrent Human Malignant Gliomas after Alkylator Chemotherapy. Cancer Res. 2006;66(8).

100. Cahill DP, Levine KK, Betensky RA, Codd PJ, Romany CA, Reavie LB, et al. Loss of the mismatch repair protein MSH6 in human glioblastomas is associated with tumor progression during temozolomide treatment. Clin Cancer Res. 2007 Apr 1;13(7):2038-45.

101. Yip S, Miao J, Cahill DP, Iafrate AJ, Aldape K, Nutt CL, et al. MSH6 mutations arise in glioblastomas during temozolomide therapy and mediate 
temozolomide resistance. Clin Cancer Res. 2009 Jul 15;15(14):4622-9.

102. Nguyen SA, Stechishin ODM, Luchman HA, Lun XQ, Senger DL, Robbins SM, et al. Novel MSH6 Mutations in Treatment-Naïve Glioblastoma and Anaplastic Oligodendroglioma Contribute to Temozolomide Resistance Independently of MGMT Promoter Methylation. Clin Cancer Res. 2014;20(18).

103. Agnihotri S, Gajadhar AS, Ternamian C, Gorlia T, Diefes KL, Mischel PS, et al. Alkylpurine-DNA-N-glycosylase confers resistance to temozolomide in xenograft models of glioblastoma multiforme and is associated with poor survival in patients. J Clin Invest. 2012 Jan;122(1):253-66.

104. Fosmark S, Hellwege S, Dahlrot RH, Jensen KL, Derand H, Lohse J, et al. APNG as a prognostic marker in patients with glioblastoma. PLoS One. 2017;12(6):e0178693.

105. Chen M-K, Hung M-C. Regulation of therapeutic resistance in cancers by receptor tyrosine kinases. Am J Cancer Res. 2016;6(4):827-42.

106. Mukherjee B, McEllin B, Camacho C V., Tomimatsu N, Sirasanagandala S, Nannepaga S, et al. EGFRvIII and DNA Double-Strand Break Repair: A Molecular Mechanism for Radioresistance in Glioblastoma. Cancer Res. 2009;69(10).

107. Valerie K, Yacoub A, Hagan MP, Curiel DT, Fisher PB, Grant S, et al. Radiation-induced cell signaling: inside-out and outside-in. Mol Cancer Ther. 2007;6(3).

108. Li H-F, Kim J-S, Waldman T. Radiation-induced Akt activation modulates radioresistance in human glioblastoma cells. Radiat Oncol. 2009 Oct 14;4:43.

109. Dittmann K, Mayer C, Fehrenbacher B, Schaller M, Raju U, Milas L, et al. Radiation-induced epidermal growth factor receptor nuclear import is linked to activation of DNA-dependent protein kinase. J Biol Chem. 2005 Sep 2;280(35):31182-9.

110. Wang S-C, Hung M-C. Nuclear translocation of the epidermal growth factor receptor family membrane tyrosine kinase receptors. Clin Cancer Res. 2009 Nov 1;15(21):6484-9.

111. Bianco J, Bastiancich C, Jankovski A, des Rieux A, Préat V, Danhier F. On glioblastoma and the search for a cure: where do we stand? Cell Mol Life Sci. 2017 Feb 17;1-16. 
112. Galli R, Binda E, Orfanelli U, Cipelletti B, Gritti A, De Vitis S, et al. Isolation and Characterization of Tumorigenic, Stem-like Neural Precursors from Human Glioblastoma. Cancer Res. 2004 Oct 1;64(19):7011-21.

113. Yuan X, Curtin J, Xiong Y, Liu G, Waschsmann-Hogiu S, Farkas DL, et al. Isolation of cancer stem cells from adult glioblastoma multiforme. Oncogene. 2004;23(58):9392-400.

114. Salmaggi A, Boiardi A, Gelati M, Russo A, Calatozzolo C, Ciusani E, et al. Glioblastoma-derived tumorospheres identify a population of tumor stemlike cells with angiogenic potential and enhanced multidrug resistance phenotype. Glia. 2006;54(8):850-60.

115. Lathia JD, Mack SC, Mulkearns-Hubert EE, Valentim CLL, Rich JN. Cancer stem cells in glioblastoma. Genes Dev. 2015 Jun 15;29(12):1203-17.

116. Schonberg DL, Lubelski D, Miller TE, Rich JN. Brain tumor stem cells: Molecular characteristics and their impact on therapy. Mol Aspects Med. 2014 Oct;39:82-101.

117. Osuka S, Van Meir EG. Overcoming therapeutic resistance in glioblastoma: the way forward. J Clin Invest. 2017 Feb 1;127(2):415-26.

118. Kaur B, Tan C, Brat DJ, Van meir EG. Genetic and hypoxic regulation of angiogenesis in gliomas. J Neurooncol. 2004 Nov;70(2):229-43.

119. McGee MC, Hamner JB, Williams RF, Rosati SF, Sims TL, Ng CY, et al. Improved intratumoral oxygenation through vascular normalization increases glioma sensitivity to ionizing radiation. Int J Radiat Oncol Biol Phys. 2010 Apr;76(5):1537-45.

120. Glass R, Synowitz M. CNS macrophages and peripheral myeloid cells in brain tumours. Acta Neuropathol. 2014 Sep 11;128(3):347-62.

121. POLIVKA JR J, POLIVKA J, HOLUBEC L, KUBIKOVA T, PRIBAN V, HES $O$, et al. Advances in Experimental Targeted Therapy and Immunotherapy for Patients with Glioblastoma Multiforme. Anticancer Res. 2017 Jan 1;37(1):21-33.

122. Neagu MR, Reardon DA. An Update on the Role of Immunotherapy and Vaccine Strategies for Primary Brain Tumors. Curr Treat Options Oncol. 2015 Nov 10;16(11):54.

123. Huang J, Liu F, Liu Z, Tang H, Wu H, Gong Q, et al. Immune Checkpoint in Glioblastoma: Promising and Challenging. Front Pharmacol. 2017;8:242. 
124. Reardon DA, Turner S, Peters KB, Desjardins A, Gururangan S, Sampson $\mathrm{JH}$, et al. A review of VEGF/VEGFR-targeted therapeutics for recurrent glioblastoma. J Natl Compr Canc Netw. 2011 Apr;9(4):414-27.

125. Field KM, Jordan JT, Wen PY, Rosenthal MA, Reardon DA. Bevacizumab and glioblastoma: Scientific review, newly reported updates, and ongoing controversies. Cancer. 2015 Apr 1;121(7):997-1007.

126. Cohen MH, Shen YL, Keegan P, Pazdur R. FDA drug approval summary: bevacizumab (Avastin) as treatment of recurrent glioblastoma multiforme. Oncologist. 2009 Nov;14(11):1131-8.

127. Balañá C, Etxaniz O, Bugés C, Martínez A. Approval denied by the European Medicines Agency (EMA) for bevacizumab in the treatment of high-grade glioma recurrence: a good idea or a grave error? Clin Transl Oncol. 2011 Mar 8;13(3):209-10.

128. Wick W, Weller M, van den Bent M, Stupp R. Bevacizumab and recurrent malignant gliomas: a European perspective. J Clin Oncol. 2010 Apr 20;28(12):e188-9; author reply e190-2.

129. Gilbert MR, Dignam JJ, Armstrong TS, Wefel JS, Blumenthal DT, Vogelbaum MA, et al. A Randomized Trial of Bevacizumab for Newly Diagnosed Glioblastoma. N Engl J Med. 2014 Feb 20;370(8):699-708.

130. Chinot OL, Wick W, Mason W, Henriksson R, Saran F, Nishikawa R, et al. Bevacizumab plus Radiotherapy-Temozolomide for Newly Diagnosed Glioblastoma. N Engl J Med. 2014 Feb 20;370(8):709-22.

131. Diaz RJ, Ali S, Qadir MG, De La Fuente MI, Ivan ME, Komotar RJ. The role of bevacizumab in the treatment of glioblastoma. J Neurooncol. 2017 Jul 19;133(3):455-67.

132. Schachter J, Ribas A, Long G V, Arance A, Grob J-J, Mortier L, et al. Pembrolizumab versus ipilimumab for advanced melanoma: final overall survival results of a multicentre, randomised, open-label phase 3 study (KEYNOTE-006). Lancet. 2017 Oct 21;390(10105):1853-62.

133. Sayour EJ, Mitchell DA. Manipulation of Innate and Adaptive Immunity through Cancer Vaccines. J Immunol Res. 2017;2017:3145742.

134. Stupp R, Taillibert S, Kanner AA, Kesari S, Steinberg DM, Toms SA, et al. Maintenance Therapy With Tumor-Treating Fields Plus Temozolomide vs Temozolomide Alone for Glioblastoma. JAMA. 2015 Dec 15;314(23):2535. 
135. Zhu P, Zhu J-J. Tumor treating fields: a novel and effective therapy for glioblastoma: mechanism, efficacy, safety and future perspectives. Chinese Clin Oncol. 2017 Aug;6(4):41-41.

136. Lok E, Swanson KD, Wong ET. Tumor treating fields therapy device for glioblastoma: physics and clinical practice considerations. Expert Rev Med Devices. 2015 Nov 2;12(6):717-26.

137. Rehman AA, Elmore KB, Mattei TA. The effects of alternating electric fields in glioblastoma: current evidence on therapeutic mechanisms and clinical outcomes. Neurosurg Focus. 2015 Mar;38(3):E14.

138. Stupp R, Heigi M, Idbaih A, Steinber D, Lhermitte B, Read W, et al. Abstract CT007: Tumor treating fields added to standard chemotherapy in newly diagnosed glioblastoma (GBM): Final results of a randomized, multi-center, phase III trial. Cancer Res. 2017;77(13).

139. U.S. Food and Drug Administration. Premarket Approval (PMA)- OPTUNE (formerly the NovoTTF-100A system) - expanded indication approval P100034S013 [Internet]. 2015 [cited 2017 Nov 7]. Available from: https://www.accessdata.fda.gov/scripts/cdrh/cfdocs/cfpma/pma.cfm?id=P $100034 \mathrm{~S} 013$

140. Alexander BM, Ba S, Berger MS, Berry DA, Cavenee WK, Chang SM, et al. Adaptive Global Innovative Learning Environment for Glioblastoma: GBM AGILE. Clin Cancer Res. 2017;

141. Ye B, Lista F, Lo Coco F, Knowles D, Offit K, Chaganti R, et al. Alterations of a zinc finger-encoding gene, BCL-6, in diffuse large-cell lymphoma. Science (80- ). 1993;262(5134).

142. Kerckaert J-P, Deweindt C, Tilly H, Quief S, Lecocq G, Bastard C. LAZ3, a novel zinc-finger encoding gene, is disrupted by recurring chromosome 3q27 translocations in human lymphomas. Nat Genet. 1993 Sep 1;5(1):66-70.

143. Baron BW, Nucifora G, McCabe N, Espinosa R, Le Beau MM, McKeithan TW, et al. Identification of the gene associated with the recurring chromosomal translocations $\mathrm{t}(3 ; 14)(\mathrm{q} 27 ; \mathrm{q} 32)$ and $\mathrm{t}(3 ; 22)(\mathrm{q} 27 ; \mathrm{q} 11)$ in B-cell lymphomas. Proc Natl Acad Sci U S A. 1993 Jun 1;90(11):5262-6.

144. Miki T, Kawamata N, Hirosawa S, Aoki N. Gene involved in the 3q27 translocation associated with B-cell lymphoma, BCL5, encodes a Kruppellike zinc-finger protein. Blood. 1994;83(1). 
145. Phan RT, Dalla-Favera R. The BCL6 proto-oncogene suppresses p53 expression in germinal-centre B cells. Nature. 2004 Dec 2;432(7017):635-9.

146. Ranuncolo SM, Polo JM, Dierov J, Singer M, Kuo T, Greally J, et al. Bcl-6 mediates the germinal center B cell phenotype and lymphomagenesis through transcriptional repression of the DNA-damage sensor ATR. Nat Immunol. 2007 Jul 10;8(7):705-14.

147. Ranuncolo SM, Polo JM, Melnick A. BCL6 represses CHEK1 and suppresses DNA damage pathways in normal and malignant B-cells. Blood Cells, Mol Dis. 2008 Jul;41(1).

148. Lenz G, Staudt LM. Aggressive Lymphomas. Schwartz RS, editor. N Engl J Med. 2010 Apr 15;362(15):1417-29.

149. Saito M, Novak U, Piovan E, Basso K, Sumazin P, Schneider C, et al. BCL6 suppression of BCL2 via Miz1 and its disruption in diffuse large B cell lymphoma. Proc Natl Acad Sci U S A. 2009 Jul 7;106(27):11294-9.

150. Nahar R, Ramezani-Rad P, Mossner M, Duy C, Cerchietti L, Geng H, et al. Pre-B cell receptor-mediated activation of BCL6 induces pre-B cell quiescence through transcriptional repression of MYC. Blood. 2011 Oct 13;118(15):4174-8.

151. Hatzi K, Melnick A. Breaking bad in the germinal center: how deregulation of BCL6 contributes to lymphomagenesis. Trends Mol Med. 2014;20(6):34352.

152. Ci W, Polo JM, Cerchietti L, Shaknovich R, Wang L, Yang SN, et al. The BCL6 transcriptional program features repression of multiple oncogenes in primary B cells and is deregulated in DLBCL. Blood. 2009;113(22).

153. Duy C, Hurtz C, Shojaee S, Cerchietti L, Geng H, Swaminathan S, et al. BCL6 enables $\mathrm{Ph}+$ acute lymphoblastic leukaemia cells to survive BCRABL1 kinase inhibition. Nature. 2011/05/20. 2011;473(7347):384-8.

154. Slone WL, Moses BS, Hare I, Evans R, Piktel D, Gibson LF, et al. BCL6 modulation of acute lymphoblastic leukemia response to chemotherapy. Oncotarget. 2016 Apr 26;7(17):23439-53.

155. Basso K, Dalla-Favera R. Roles of BCL6 in normal and transformed germinal center B cells. Immunol Rev. 2012 May;247(1):172-83.

156. Huynh KD, Fischle W, Verdin E, Bardwell VJ. BCoR, a novel corepressor involved in BCL-6 repression. Genes Dev. 2000 Jul 15;14(14):1810-23. 
157. Ghetu AF, Corcoran CM, Cerchietti L, Bardwell VJ, Melnick A, Privé GG. Structure of a BCOR Corepressor Peptide in Complex with the BCL6 BTB Domain Dimer. Vol. 29, Molecular Cell. 2008.

158. Li J, Wang J, Wang J, Nawaz Z, Liu JM, Qin J, et al. Both corepressor proteins SMRT and $\mathrm{N}-\mathrm{CoR}$ exist in large protein complexes containing HDAC3. EMBO J. 2000;19(16):4342-50.

159. Chen JD, Evans RM. A transcriptional co-repressor that interacts with nuclear hormone receptors. Nature. 1995 Oct 5;377(6548):454-7.

160. Gearhart MD, Corcoran CM, Wamstad JA, Bardwell VJ. Polycomb Group and SCF Ubiquitin Ligases Are Found in a Novel BCOR Complex That Is Recruited to BCL6 Targets. Mol Cell Biol. 2006 Sep 15;26(18):6880-9.

161. Sánchez C, Sánchez I, Demmers JAA, Rodriguez P, Strouboulis J, Vidal M. Proteomics Analysis of Ring1B/Rnf2 Interactors Identifies a Novel Complex with the Fbxl10/Jhdm1B Histone Demethylase and the Bcl6 Interacting Corepressor. Mol Cell Proteomics. 2007 May;6(5):820-34.

162. Farcas AM, Blackledge NP, Sudbery I, Long HK, McGouran JF, Rose NR, et al. KDM2B links the Polycomb Repressive Complex 1 (PRC1) to recognition of CpG islands. Elife. 2012 Dec 18;1:e00205.

163. Gao Z, Zhang J, Bonasio R, Strino F, Sawai A, Parisi F, et al. PCGF Homologs, CBX Proteins, and RYBP Define Functionally Distinct PRC1 Family Complexes. Mol Cell. 2012 Feb 10;45(3):344-56.

164. Hatzi K, Jiang Y, Huang C, Garrett-Bakelman F, Gearhart MD, Giannopoulou EG, et al. A Hybrid Mechanism of Action for BCL6 in B Cells Defined by Formation of Functionally Distinct Complexes at Enhancers and Promoters. Cell Rep. 2013;4(3):578-88.

165. Karagianni P, Wong J. HDAC3: taking the SMRT-N-CoRrect road to repression. Oncogene. 2007 Aug 13;26(37):5439-49.

166. Parekh S, Polo JM, Shaknovich R, Juszczynski P, Lev P, Ranuncolo SM, et al. BCL6 programs lymphoma cells for survival and differentiation through distinct biochemical mechanisms. Blood. 2007 Sep 15;110(6):2067-74.

167. Tunyaplin C, Shaffer AL, Angelin-Duclos CD, Yu X, Staudt LM, Calame KL. Direct repression of prdm1 by Bcl-6 inhibits plasmacytic differentiation. J Immunol. 2004 Jul 15;173(2):1158-65.

168. Mendez LM, Polo JM, Yu JJ, Krupski M, Ding BB, Melnick A, et al. CtBP Is 
an Essential Corepressor for BCL6 Autoregulation. Mol Cell Biol. 2008 Apr 1;28(7):2175-86.

169. Niu H, Ye BH, Dalla-Favera R. Antigen receptor signaling induces MAP kinase-mediated phosphorylation and degradation of the BCL-6 transcription factor. Genes Dev. 1998 Jul 1;12(13):1953-61.

170. Bereshchenko OR, Gu W, Dalla-Favera R. Acetylation inactivates the transcriptional repressor BCL6. Nat Genet. 2002 Oct 28;32(4):606-13.

171. Ye BH, Cattoretti G, Shen Q, Zhang J, Hawe N, Waard R de, et al. The BCL-6 proto-oncogene controls germinal-centre formation and Th2-type inflammation. Nat Genet. 1997 Jun;16(2):161-70.

172. Muramatsu M, Kinoshita K, Fagarasan S, Yamada S, Shinkai Y, Honjo T. Class switch recombination and hypermutation require activation-induced cytidine deaminase (AID), a potential RNA editing enzyme. Cell. 2000 Sep 1;102(5):553-63.

173. Klein U, Casola S, Cattoretti G, Shen Q, Lia M, Mo T, et al. Transcription factor IRF4 controls plasma cell differentiation and class-switch recombination. Nat Immunol. 2006 Jul 11;7(7):773-82.

174. Nurieva RI, Chung Y, Martinez GJ, Yang XO, Tanaka S, Matskevitch TD, et al. Bcl6 mediates the development of T follicular helper cells. Science. 2009 Aug 21;325(5943):1001-5.

175. Johnston RJ, Poholek AC, DiToro D, Yusuf I, Eto D, Barnett B, et al. Bcl6 and Blimp-1 Are Reciprocal and Antagonistic Regulators of T Follicular Helper Cell Differentiation. Science (80- ). 2009 Aug 21;325(5943):1006-10.

176. Yu D, Rao S, Tsai LM, Lee SK, He Y, Sutcliffe EL, et al. The Transcriptional Repressor Bcl-6 Directs T Follicular Helper Cell Lineage Commitment. Immunity. 2009 Sep;31(3):457-68.

177. Crotty S. T follicular helper cell differentiation, function, and roles in disease. Immunity. 2014 Oct 16;41(4):529-42.

178. Huang C, Hatzi K, Melnick A. Lineage-specific functions of Bcl-6 in immunity and inflammation are mediated by distinct biochemical mechanisms. Nat Immunol. 2013;14(4):380-8.

179. Cardenas MG, Yu W, Beguelin W, Teater MR, Geng H, Goldstein RL, et al. Rationally designed BCL6 inhibitors target activated B cell diffuse large B cell lymphoma. J Clin Invest. 2016 Sep 1;126(9):3351-62. 
180. Walker SR, Liu S, Xiang M, Nicolais M, Hatzi K, Giannopoulou E, et al. The transcriptional modulator BCL6 as a molecular target for breast cancer therapy. Oncogene. 2015 Feb 26;34(9):1073-82.

181. Wu Q, Liu X, Yan H, He Y, Ye S, Cheng X, et al. B-cell lymphoma 6 protein stimulates oncogenicity of human breast cancer cells. BMC Cancer. 2014;14:418.

182. Yu J-M, Sun W, Hua F, Xie J, Lin H, Zhou D-D, et al. BCL6 induces EMT by promoting the ZEB1-mediated transcription repression of E-cadherin in breast cancer cells. Cancer Lett. 2015 Sep;365(2):190-200.

183. Deb D, Rajaram S, Larsen JE, Dospoy PP, Marullo R, Li L-S, et al. Combination therapy targeting BCL6 and phospho-STAT3 defeats intratumor heterogeneity in a subset of non-small cell lung cancers. Cancer Res. 2017;

184. Wang Y-Q, Xu M-D, Weng W-W, Wei P, Yang Y-S, Du X. BCL6 is a negative prognostic factor and exhibits pro-oncogenic activity in ovarian cancer. Am J Cancer Res. 2015;5(1):255-66.

185. Sena P, Mariani F, Benincasa M, Ponz De Leon M, Di Gregorio C, Mancini $\mathrm{S}$, et al. Morphological and quantitative analysis of BCL6 expression in human colorectal carcinogenesis. Oncol Rep. 2013 Nov 13;31(1).

186. Ruggieri S, Tamma R, Marzullo A, Annese T, Marinaccio C, Errede M, et al. Translocation of the proto-oncogene Bcl-6 in human glioblastoma multiforme. Cancer Lett. 2014 Oct 10;353(1):41-51.

187. Xu L, Chen Y, Dutra-Clarke M, Mayakonda A, Hazawa M, Savinoff SE, et al. BCL6 promotes glioma and serves as a therapeutic target. Proc Natl Acad Sci U S A. 2017 Apr 11;114(15):3981-6.

188. Andrews S. Babraham Bioinformatics - FastQC A Quality Control tool for High Throughput Sequence Data [Internet]. 2010. Available from: https://www.bioinformatics.babraham.ac.uk/projects/fastqc/

189. Bolger AM, Lohse M, Usadel B. Trimmomatic: a flexible trimmer for Illumina sequence data. Bioinformatics. 2014 Aug 1;30(15):2114-20.

190. Bray NL, Pimentel H, Melsted P, Pachter L. Near-optimal probabilistic RNA-seq quantification. Nat Biotechnol. 2016 Apr 4;34(5):525-7.

191. Aken BL, Ayling S, Barrell D, Clarke L, Curwen V, Fairley S, et al. The Ensembl gene annotation system. Database. 2016 Jun 23;2016:baw093. 
192. Soneson C, Love MI, Robinson M. Differential Analyses for RNA-seq: transcript-level estimates improve gene-level inferences. F1000Research. $2015 ; 4$.

193. Love MI, Huber W, Anders S. Moderated estimation of fold change and dispersion for RNA-seq data with DESeq2. Genome Biol. 2014 Dec 5;15(12):550.

194. Subramanian A, Tamayo P, Mootha VK, Mukherjee S, Ebert BL, Gillette MA, et al. Gene set enrichment analysis: a knowledge-based approach for interpreting genome-wide expression profiles. Proc Natl Acad Sci U S A. 2005 Oct 25;102(43):15545-50.

195. Liberzon A, Subramanian A, Pinchback R, Thorvaldsdottir H, Tamayo P, Mesirov JP. Molecular signatures database (MSigDB) 3.0. Bioinformatics. 2011 Jun 15;27(12):1739-40.

196. Chang CC, Ye BH, Chaganti RS, Dalla-Favera R. BCL-6, a POZ/zinc-finger protein, is a sequence-specific transcriptional repressor. Proc Natl Acad Sci U S A. 1996 Jul 9;93(14):6947-52.

197. Ahmad KF, Melnick A, Lax S, Bouchard D, Liu J, Kiang C-L, et al. Mechanism of SMRT Corepressor Recruitment by the BCL6 BTB Domain. Mol Cell. 2003;12(6):1551-64.

198. Cerchietti LC, Ghetu AF, Zhu X, Da Silva GF, Zhong S, Matthews M, et al. A Small-Molecule Inhibitor of BCL6 Kills DLBCL Cells In Vitro and In Vivo. Cancer Cell. 2010 Apr 13;17(4):400-11.

199. L.C. Cerchietti R. Shaknovich, K. Hatzi, J.M. Polo, A. Chadburn, S.F. Dowdy, A. Melnick SNY. A peptomimetic inhibitor of BCL6 with potent antilymphoma effects in vitro and in vivo. Blood. 2009;113:3397-405.

200. Granadino-Roldán JM, Obiol-Pardo C, Pinto M, Garzón A, Rubio-Martínez J. Molecular dynamics analysis of the interaction between the human BCL6 BTB domain and its SMRT, NcoR and BCOR corepressors: The quest for a consensus dynamic pharmacophore. J Mol Graph Model. 2014;50:142-51.

201. Jones NM, Rowe MR, Shepherd PR, McConnell MJ. Targeted inhibition of dominant PI3-kinase catalytic isoforms increase expression of stem cell genes in glioblastoma cancer stem cell models. Int J Oncol. 2016;49(1):207-16.

202. Allman D, Jain A, Dent A, Maile R, Selvaggi T, Kehry M, et al. BCL-6 expression during B-cell activation. Blood. 1996;87(12). 
203. Yang JA, Tubo NJ, Gearhart MD, Bardwell VJ, Jenkins MK. Cutting Edge: Bcl6-Interacting Corepressor Contributes to Germinal Center T Follicular Helper Cell Formation and B Cell Helper Function. J Immunol. 2015;194(12).

204. Pasqualucci L, Migliazza A, Basso K, Houldsworth J, Chaganti RSK, DallaFavera R. Mutations of the BCL6 proto-oncogene disrupt its negative autoregulation in diffuse large B-cell lymphoma. 2003;101:2914-23.

205. Hatzi K, Nance JP, Kroenke MA, Bothwell M, Haddad EK, Melnick A, et al. BCL6 orchestrates Tfh cell differentiation via multiple distinct mechanisms. J Exp Med. 2015;212(4).

206. Wegner W, Burckhardt G, Henjakovic M. Transcriptional regulation of human organic anion transporter 1 by B-cell CLL/lymphoma 6. Am J Physiol - Ren Physiol. 2014;307(11).

207. Kubista M, Andrade JM, Bengtsson M, Forootan A, Jonák J, Lind K, et al. The real-time polymerase chain reaction. Mol Aspects Med. 2006 Apr 1;27(2-3):95-125.

208. Migocka-Patrzałek M, Makowiecka A, Nowak D, Mazur AJ, Hofmann WA, Malicka-Błaszkiewicz M. $\beta$ - and $\gamma$-Actins in the nucleus of human melanoma A375 cells. Histochem Cell Biol. 2015 Nov;144(5):417-28.

209. Hendzel MJ. The F-act's of nuclear actin. Curr Opin Cell Biol. 2014 Jun 1;28:84-9.

210. de Lanerolle P, Serebryannyy L. Nuclear actin and myosins: Life without filaments. Nat Cell Biol. 2011 Nov 2;13(11):1282-8.

211. Love MI, Huber W, Anders S. Moderated estimation of fold change and dispersion for RNA-seq data with DESeq2. Genome Biol. 2014 Dec 5;15(12):550.

212. Yan D, Wallingford JB, Sun T-Q, Nelson AM, Sakanaka C, Reinhard C, et al. Cell autonomous regulation of multiple Dishevelled-dependent pathways by mammalian Nkd. Proc Natl Acad Sci. 2001 Mar 27;98(7):3802-7.

213. Wharton KA, Zimmermann G, Rousset R, Scott MP. Vertebrate Proteins Related to Drosophila Naked Cuticle Bind Dishevelled and Antagonize Wnt Signaling. Dev Biol. 2001 Jun 1;234(1):93-106.

214. Farlie P, Reid C, Wilcox S, Peeters J, Reed G, Newgreen D. Ypel1: a novel nuclear protein that induces an epithelial-like morphology in fibroblasts. 
Genes Cells. 2001 Jul;6(7):619-29.

215. Liberzon A, Birger C, Thorvaldsdottir H, Ghandi M, Mesirov JP, Tamayo P. The Molecular Signatures Database Hallmark Gene Set Collection. Cell Syst. 2015;1(6):417-25.

216. Balkwill F. Tumour necrosis factor and cancer. Nat Rev Cancer. 2009 May 3;9(5):361-71.

217. Laplante M, Sabatini DM. mTOR Signaling. Cold Spring Harb Perspect Biol. 2012 Feb 1;4(2):a011593.

218. Ribas de Pouplana L, Schimmel P. Aminoacyl-tRNA synthetases: potential markers of genetic code development. Trends Biochem Sci. 2001 Oct 1;26(10):591-6.

219. Polo JM, Juszczynski P, Monti S, Cerchietti L, Ye K, Greally JM, et al. Transcriptional signature with differential expression of BCL6 target genes accurately identifies BCL6-dependent diffuse large B cell lymphomas. Proc Natl Acad Sci U S A. 2007 Feb 27;104(9):3207-12. 


\section{$8 \quad$ Appendix}

The oncogenic transcription factor BCL6 drives survival of glioblastoma and is up-regulated in response to DNA damage.

Marie-Sophie Fabre* ${ }^{1,2}$, Nicole M. Jones* ${ }^{1,2}$ Tania L. Slatter $^{3}$, Rosemary Gordon ${ }^{1}$, Ahmad Taha ${ }^{4}$, Janice A. Royds ${ }^{3}$, Noelyn Hung ${ }^{3}$, M. Leticia Castro ${ }^{1}$, Ari M. Melnick ${ }^{5}$, and Melanie J. McConnell ${ }^{1,2}$

1. Centre for Biodiscovery and School of Biological Sciences, Victoria University of Wellington, New Zealand

2. Malaghan Institute of Medical Research, Wellington, New Zealand

3. Department of Pathology, Dunedin School of Medicine, University of Otago, New Zealand

4. Neurosugery, Southern District Health Board, Dunedin, New Zealand

5. Weill Cornell Medical College, New York, NY USA

* These authors made an equal contribution

Key words: BCL6; glioblastoma; DNA damage; transcription factor; cell death

Corresponding Author: Melanie J. McConnell, PhD. Centre for Biodiscovery and School of Biological Sciences, Victoria University of Wellington. PO Box 600, Wellington 6140, New Zealand.

Phone +64 44635233 x8136; fax +64 4463 5331. melanie.mcconnell@,vuw.ac.nz 


\begin{abstract}
The prognosis for people with the high-grade brain tumor glioblastoma is very poor, due largely to poor cell death in response to therapy. The transcription factor BCL6 was identified as a transcription factor mediating survival in glioblastoma. Expression was observed in glioblastoma tumour specimens and cell lines. Treatment with ionizing radiation, temozolomide or doxorubicin induced endogenous BCL6 expression, which was shown to be able to bind DNA and was transcriptionally active. BCL6 expression and activity were reduced by siRNA, peptide inhibitor, small molecule inhibitor or dominant negative BCL6 mutant in a panel of glioblastoma cell lines. This caused a small increase in apoptosis, but a profound loss in proliferative ability. Both transcriptional and pro-survival activity were blocked by inhibitors of protein-protein interaction in the BTB/POZ domain of BCL6, but co-repressor expression was not required for BCL6 activity in glioblastoma. Instead it appears that BCL6 induced by therapy could activate transcription. Together these data demonstrate that BCL6 is an active transcription factor in glioblastoma, that it drives survival of cells, and that levels of BCL6 increased after DNA damage, potentially increasing the survival rate of therapytreated cells. This makes BCL6 an excellent therapeutic target in glioblastoma. By increasing sensitivity to standard DNA damaging therapy, BCL6 inhibitors have real potential to improve the outcome for people with this terrible disease, who currently have no effective options for therapy.
\end{abstract}




\section{Introduction}

The prognosis for people diagnosed with the WHO grade IV brain tumor glioblastoma is very poor, due largely to the lack of response to therapy. The goldstandard therapy for glioblastoma is surgery to debulk the tumor, followed by fractionated radiation and temozolomide chemotherapy [1]. This aims to induce significant DNA damage to the remaining, non-resected tumor - both single and double-stranded DNA breaks from radiation-induced radical species, and alkylation of purine residues by temozolomide. The expected cellular response to this DNA damage should be apoptosis. In glioblastoma, this does not occur - there is little or no apoptosis in response to therapy [2], so damaged cells continue to proliferate, exacerbating the mutagenic and genome instability effects of DNA damaging therapy. New approaches in glioblastoma such as targeted therapy and immunotherapy continue to be developed, but these are expensive, experimental, and have had very limited success [3]. If the block to cell death could be identified, glioblastoma could be sensitized to DNA damage induced by standard therapies, which would have an immediate impact on patient outcome.

Cell death blockade in response to DNA damage is observed during B-cell maturation, driven by the transcription factor BCL6. BCL6 dimers bind DNA using six zinc fingers at the $\mathrm{C}$-terminus, and recruit co-repressors and chromatin remodeling machinery via the BTB domain to target gene loci. BCL6 is normally expressed in germinal center B-cells during class switch recombination and somatic hyper-mutation, where it represses expression of cell cycle checkpoint and apoptosis genes. This prevents the usual cellular response to double-stranded breaks, allowing cells to successfully break and rearrange immune genes to generate unique immune receptors. Due to this anti-apoptotic activity BCL6 is a strong oncogene, with ectopic expression in B-cells a key driver event in lymphoma [4, 5].

Increasingly BCL6 has been found expressed in solid malignancies, including squamous cell carcinoma [6] colorectal [7] gallbladder [8], and breast cancer [9]. In most cases, BCL6 expression is associated with poor prognosis and worse outcome, although not always - BCL6 can suppress tumorigenesis in medulloblastoma [10] and is associated with a better prognosis in a subset of gastric lymphoma [11]. A recent publication showed that the BCL6 locus was translocated and expressed in a 
subset of glioblastoma with IDH1 expression [12] and very recently, BCL6 expression was observed in glioblastoma where it correlated with increased expression of the Axl kinase [13].

Apoptotic and cell death defects are critical in survival of glioblastoma and resistance to treatment, and these defects could be driven by BCL6. Apoptosis induced by chemotherapeutic agents was prevented by BCL6 over-expression in lymphoma cell lines, in part through enhancement of the antioxidant defense systems [14]. BCL6 is central to a receptor tyrosine kinase inhibitor (TKI) drugresistance pathway, and BCL6 inhibition eradicated drug-resistant leukemiainitiating cells [15]. In GBM, BCL6 expression can be found associated with defects in apoptosis - for example, BCL6 and the BCL6 target gene EP300 [16] are among anti-apoptosis genes in a signature of survival in primary glioblastoma [17].

Here, we confirmed that glioblastoma express BCL6, and further demonstrated that DNA damaging therapy up-regulated BCL6 expression in vitro and in intra-cranial tumor model in vivo. BCL6 was transcriptionally active, and inhibition of BCL6 activity in a panel of glioblastoma cells showed that it was essential for proliferation and survival of glioblastoma.

\section{Material and methods}

2.1 Tumour immunohistochemistry: Sixty-two glioblastomas were obtained from neurosurgical units in Dunedin, New Zealand. The study had National ethics approval (MEC/08/02/016/AM01) and all patients provided written informed consent. Tumors had mutations in TP53 identified by sequencing DNA extracted from frozen material. Tissue sections from paraffin-embedded material were subjected to heat-mediated antigen retrieval. BCL6 staining used the PG-B6p primary antibody (Dako, Glostrup, Denmark). MGMT staining used the MT3.1 primary antibody (Abcam, Cambridge, UK). Positive cells were identified using EDL (Dako) and DAB. Positive cells were counted in at least 10-high-powered fields (x400 magnification) using the Aperio Scancope CS digital pathology system (Aperio, Vista, CA, USA) and the percentage of positive cells per total cells measured. MGMT positive tumours had $>30 \%$ positive tumor cells. 
2.2 Cell lines: Human GBM lines LN18, U87-MG and T98G were obtained from ATCC and used within 20 passages. NZG-1003 and NZ-G0906 primary GBM cell lines were previously derived from primary tumors in our laboratory [18, 19]. Raji cells were a gift from Ian Morison, University of Otago, New Zealand. All cells were maintained at $37^{\circ} \mathrm{C} / 5 \% \mathrm{CO}_{2}$ and cultured in RPMI-1640 with $10 \%$ FBS without antibiotics. Regular PCR testing (e-Myco, Intron BioTechnology, Korea) showed cultures were mycoplasma free.

2.3 Intra-cranial tumors: Male C57/Bl6 mice, 8 weeks old, weighing 25-30 g, were injected intra-cranially with 25,000 viable GL261 cells as previously described, then exposed to 10Gy of whole-brain ionizing radiation, with 7Gy delivered to the brain [20] at the onset of symptoms (at least two consecutive days of weight loss). They were culled by $\mathrm{CO}_{2}$ inhalation either 24 or 48 hours after treatment and cardiac perfusion of saline performed before collection of brain tissue. Untreated tumor bearing mice were used as controls. All procedures were approved by the Victoria University of Wellington Animal Ethics committee, approval 2012R7M. Brain were then frozen and kept at $-80 \mathrm{C}$ until sectioning.

2.4 Drugs: Doxorubicin (Merck, Billercia, USA) was dissolved at $10 \mathrm{mM}$ in PBS. Cells were treated with 1-3 mM and harvested at 1-3 days. TMZ was dissolved in PBS at $0.33 \mathrm{mg} / \mathrm{mL}$, and cells treated with $10 \mu \mathrm{M}$ TMZ every 2 days for 7 treatments. Cells were harvested 2 days after the last treatment. Cells received ionizing radiation from a cesium-137 source (Gammacell 3000 Elan, Theratronics, Ottowa, CA). After irradiation, cells were incubated for 24 hours before harvest. The peptide mimetic inhibitor RI-BPI[21] was dissolved in sterile pre-gassed water pH6.2, at $50^{\circ} \mathrm{C}$ and used at $2 \mathrm{mM}$. The small molecule inhibitor FX1[22] was dissolved in DMSO at $25 \mathrm{mM}$.

2.5 Antibodies: Mouse monoclonal anti Bcl-6 D8, anti-mouse IgG-HRP and antirabbit IgG-HRP were from Santa Cruz Biotechnology, (Santa Cruz, CA); mouse monoclonal anti- $\alpha$-Tubulin Clone B-5-1-2 from Sigma-Aldrich (Auckland, NZ), goat anti-mouse Alexa 488 from Invitrogen (ThermoFisher Scientific, Auckland, NZ); FITC anti-active Caspase3 and annexinV-APC (BD Pharmingen, Auckland, NZ); mouse monoclonal anti-BCOR, mouse monoclonal anti-NCOR2 (SMRT), and mouse monoclonal anti- $\beta$-actin were from Sigma-Aldrich (Auckland, NZ); goat 
polyclonal anti-BCOR and rabbit polyclonal anti-NCOR were from Abcam (Cambridge, UK); mouse monoclonal anti-SMRT from Gene'Tex (Irvine, CA); mouse monoclonal anti-RNA polymerase II from EMD-Millipore (Auckland, NZ) 2.6 Western Blotting: Soluble protein was extracted into lysis buffer $(140 \mathrm{mM} \mathrm{NaCl}$, $50 \mathrm{mM}$ Tris $\mathrm{pH}$ 7.5-8, 1\% triton, protease inhibitor (Complete EDTA free, Roche, Auckland NZ)) and quantified using the DC assay (Bio-Rad, Auckland NZ). Forty microgram of protein was electrophoresed through 10\% SDS PAGE and transferred to PVDF membrane (Bio-Rad, Auckland, NZ). After blocking in 3\% skim milk at room temperature, upper part of the membrane was incubated with 1:500 (3\% skim milk) of anti-BCl6 monoclonal antibody D8 and lower part with 1:2,000 (3\% skim milk) of anti-tubulin. Goat anti-mouse IgG HRP secondary antibody was used at 1:10,000 ( $3 \%$ skim milk). Detection by enhanced chemiluminescence (Ultrasignal ECL kit, Pierce) was imaged with Gel Logic 4000 PRO Imaging System (Carestream, Rochester, NY USA). Raji lysate was a positive control for BCL6 expression. Corepressor blots were done as above, but whole cell lysate electrophoresed through 6\% acrylamide gels, and blocked in 5\% BSA. All corepressor primary antibodies were used at 1:1000 dilution. ECL detection used Western Lightning Pro (Perkin Elmer, Waltham MA), imaged on the Amersham Imager 600 (GE Healthcare Life Sciences, Auckland, NZ).

2.7 Immunofluorescence: Cells grown on sterile glass coverslips were fixed 15 minutes in $4 \%$ paraformaldehyde, then permeabilized 15 minutes on ice in $0.1 \%$ Triton X100 in PBS. Samples were blocked in 3\% BSA in PBS 1 hour then anti-BCL6 antibody (1:50) was added and incubated $4^{\circ} \mathrm{C}$ overnight. Coverslips were washed in PBS then incubated 1:500 anti-mouse Alexa 488 secondary antibody, 3\% BSA in PBS, room temperature 1 hour. After washing, samples were mounted by inversion onto mounting medium on glass slides. Images were acquired using a BX51 compound microscope (Olympus, Auckland NZ). For analysis of tumors, slides were thawed at room temperature for 10 minutes then rehydrated in PBS for 10 minutes. Non-specific binding was prevented by blocking antigenic sites for 30 minutes in PBS containing 1\% FBS. A mouse monoclonal anti- BCL-6 antibody (Dako PG B6p at 1/20 or D8, at 1/50) was diluted in incubation buffer (PBS containing $1 \% \mathrm{FBS}$ and $3 \%$ triton $\mathrm{X}-100$ ) and applied overnight at 4C. Slides were then washed 3x 15 minutes in PBS. A goat anti mouse IgG labelled with AF488 (A- 
11029, 1/250 dilution) was used as a secondary antibody and incubated for 1 hour at room temperature. After 3 washes in PBS, slides were mounted using ProLong ${ }^{\text {TM }}$ Gold Antifade Mount (ThermoFisher Scientific, Auckland, NZ).

2.8 Transfection: pcDNA3-hBCL6DZNF, pcDNA3-GFP BCL6 ${ }_{4}$-tkLUC, and pGL3 control plasmids were purified using PureLink ${ }^{\circledR}$ HiPure Plasmid Filter Maxiprep Kit (Invitrogen, Auckland NZ). All cells were transfected using Viafect (Promega, Auckland NZ) at 70\% confluence following manufacturers instructions, and cells were harvested at 24-48 hrs. siRNA against BCL6 and control (Santa Cruz Biotechnology, Santa Cruz, CA, USA) were used according to the manufacturers siRNA gene silencing protocol. For hypoxic transfection, cells were pre-incubated in $0.5 \%$ oxygen for 24 hours, then transfected and replaced into $0.5 \%$ oxygen for an additional 24 hours.

2.9 Clonogenic assay: Transfected cells were flow sorted according to GFP status (Influx, BD Biosciences) then 400, 200 and 100 GFP positive or negative cells were seeded into $10 \mathrm{~cm}$ dishes and incubated for 2 weeks. Colonies were washed twice with PBS followed by fixation with neutral buffered formalin, $6 \% \mathrm{v} / \mathrm{v}$ for 30 minutes followed by overnight staining with $0.5 \%$ methylene blue, on a rocking platform. Plates were washed in cold tap water until the water ran clear, drained and allowed to dry. Plates were scanned (GE ImageScanner III) and colonies $>50$ cells were counted using Fiji (ImageJ2) software. Plating efficiency and surviving fractions were calculated as follows: Plating efficiency $(\mathrm{PE})=$ number of colonies counted / number of colonies plated. Surviving fraction $(\mathrm{SF})=\mathrm{PE}$ sample / PE control.

2.10 Cell death and apoptosis: Cells were washed in PBS+1\% BSA with $50 \mathrm{ng} / \mathrm{mL}$ PI, and PI positive cells were identified by standard flow cytometry techniques using the LSRII flow cytometer with FACSDiva 6.2 acquisition software (BD Biosciences, Auckland, NZ). Data were analysed using Flowjo software (Flowjo, Ashland, OR, USA). Annexin V positive cells were detected using either AnxVFITC or AnxV-APC (ThermoFisher Scientific, Auckland NZ). For Caspase3 activation, cells were trypsinised, washed in PBS then fixed and permeabilised using the FoxP3 Fixation/Permeabilisation Concentrate and Diluent Reagent (e- 
Biosciences, San Diego, CA). Fixed-permeabilised cells were stained overnight with FITC anti-Active Caspase3 apoptosis kit (BD Pharmingen, Auckland NZ) then washed and analysed.

2.11 Luciferase reporter assay: Luciferase activity was assessed with the Promega Luciferase Assay System (Madison, WI). Cells were washed in PBS and then lysed and scraped in cell culture lysis reagent $(25 \mathrm{mM}$ Tris-phosphate, $2 \mathrm{mM}$ Dithiothreitol, $2 \mathrm{mM}$ 1,2-diaminocyclohexane-N,N,N',N,-tetraacetic acid, 10\% (v/v) glycerol, 1\% (v/v) Triton X-100). Lysate was added to a 96 well solid white flat bottom plate (Corning, NY). Luciferase assay reagent (LAR) was prepared according to the manufacturer's instructions. LAR was injected into the plate and read by the Tecan Infinite M1000 Pro Plate Reader (Männedorf, Switzerland). Light values were normalized to cell counts (luciferase value/number of cells) taken before lysis of the cells and then relative change (double transfection or treated value/single transfected untreated value) value in light compared to untreated single-transfected control was calculated.

2.12 Quantitative Reverse Transcriptase PCR( q-RT-PCR): RNA was extracted using the Zymo Quick RNA MiniPrep kit (Zymo Research, Irvine, CA) according to the manufacturer's instructions. RNA was quantified with the Qubit RNA high sensitivity assay (Life Technologies, Auckland, NZ) according to the manufacturer's instructions. Reverse transcription was performed using the iScript cDNA synthesis kit (BioRad, Auckland, NZ) according to the manufacturer's instructions, with 250 ng of RNA in each reaction. qPCR was performed with KAPA SYBR® FAST Universal One-Step qRT-PCR Kit (KAPA Biosystems, Wilmington, MA) and Qiagen Quantitect Primer assays (Qiagen, Hilden, Germany) for BCL6, BCOR, HPRT, NCOR1, and NCOR2 (SMRT) with $4 \mu \mathrm{l}$ cDNA per reaction. DCt was calculated relative to HPRT.

2.13 Nuclear Extract preparation and Electrophoretic Mobility Shift Assay (EMSA): Nuclear extracts were prepared from cells with the Buffer A and Buffer B system[23] (Buffer A:10 mM HEPES-KOH (pH 7.9); $1.5 \mathrm{mM} \mathrm{MgCl}_{2} ; 10 \mathrm{mM} \mathrm{KCl} ; 0.5 \mathrm{mM}$ Dithiothreitol). Buffer B: 20 mM HEPES-KOH (pH 7.9), $420 \mathrm{mM} \mathrm{NaCl}, 1.5 \mathrm{mM}$ $\mathrm{MgCl}_{2} 0.2 \mathrm{mM}$ EDTA, $0.5 \mathrm{mM}$ Dithiothreitol) and were prepared fresh for each 
EMSA performed. For EMSA, sequence-specific 3'-biotin labeled oligonucleotides with the native binding site for BCL6[24] were purchase from Integrated DNA Technologies (Singapore): 5'-GAAAATTCCTAGAAAGCATA-3'. EMSA procedure was done using the Lightshift Chemiluminescent EMSA Kit (Thermo Scientific, Auckland, NZ) according to the manufacturer's recommendation. In brief, $4 \mu \mathrm{g}$ of nuclear extract was incubated with $20 \mathrm{fmol}$ of biotin-labeled probe, 50 $\mathrm{ng} / \mu \mathrm{l}$ poly dI.dC (in $10 \mathrm{mM}$ Tris and $1 \mathrm{mM}$ EDTA, $\mathrm{pH} 7.5$ ), 2.5\% glycerol, $5 \mathrm{mM}$ $\mathrm{MgCl}_{2}, 0.05 \% \mathrm{NP}-40$ and $2 \mu \mathrm{l}$ of $10 \mathrm{x}$ Binding Buffer in a total volume of $20 \mu \mathrm{l}$ for $20 \mathrm{~min}$ at room temperature. For the competition assay, 4 pmol of identical unlabeled competitor DNA was added to this reaction. For the supershift assay, 2 $\mu \mathrm{g}$ of antibody was added. After incubation, probes were loaded onto a $6 \%$ native polyacrylamide gel, electrophoresed at $100 \mathrm{~V}$ until the bromophenol blue dye had migrated approximately two-thirds to three-quarters down the gel and further blotted crosslinked on a positively charged nylon membrane (Sigma Aldrich, Auckland, NZ). Lastly, biotin-labeled DNA protein complexes were detected by chemiluminescence on Amersham Imager 600 (GE Healthcare Life Sciences, Auckland, NZ), using the Lightshift Chemiluminescent EMSA Kit reagents (Thermo Fisher, Auckland, NZ).

2.14 Chromatin Immunoprecipitation: Cells were fixed in 1\% formaldehyde for $10 \mathrm{~min}$ at room temperature. The crosslinking reaction was stopped by addition of $125 \mathrm{mM}$ glycine and cells were washed twice in cold PBS and scraped and pelleted in cold PBS. Nuclear preparation was performed by addition of Swelling Buffer $(50 \mathrm{mM}$ Hepes-KOH (pH 7.5), 140 mM NaCl, 10\% glycerol, 1 mM EDTA (pH 8.0), 0.5\% IGEPAL, $0.25 \%$ Triton X-100), nuclei were pelleted and washed with Washing Buffer (10 mM Tris-Cl (pH 8.0), $200 \mathrm{mM} \mathrm{NaCl}, 1 \mathrm{mM}$ EDTA (pH 8.0), $0.5 \mathrm{mM}$ EGTA (pH 8.0)). Isolated nuclei were resuspended in Shearing Buffer (50 mM Tris$\mathrm{Cl}(\mathrm{pH}$ 8.1), 0.1\% SDS, $10 \mathrm{mM}$ EDTA ( $\mathrm{pH} 8.0)$ ) and sonicated to generate fragments of DNA $<400$ bp. Sonicated nuclei were incubated with either BCL6 (N3), RNA polymerase II or $\beta$-actin antibodies pre-bound to Protein G Dynabeads (Invitrogen, Auckland, NZ) in RIPA buffer $(50 \mathrm{mM} \mathrm{NaCl}, 1 \%$ IGEPAL, $0.5 \% \mathrm{Na}$ DOC, $0.1 \%$ SDs, $50 \mathrm{mM}$ Tris-Cl (pH 8.0), 5 mM EDTA (pH 8.0) overnight at $4{ }^{\circ} \mathrm{C}$. Immunocomplexes were recovered by magnet and washed twice with RIPA buffer, followed by increasing stringency ChIP wash buffers $(150 \mathrm{mM} \mathrm{NaCl}, 250 \mathrm{mM}$ 
$\mathrm{NaCl}, 250 \mathrm{mM} \mathrm{LiCl})$. Immunocomplexes were eluted by adding elution buffer (1\% SDS, $100 \mathrm{mM} \mathrm{NaHCO}$ ) and cross-linking was reverted by addition of $300 \mathrm{mM}$ $\mathrm{NaCl}$ and incubation at $65^{\circ} \mathrm{C}$ overnight. DNA was purified with the QIAQuick PCR Purification Kit (Qiagen, Hilden, Germany) according to the manufacturer's instructions. The ChIP product was used as template for RT-PCR reactions using SYBR Green (KAPA Biosystems, Wilmington, MA) and primers for BCL6 Exon 1, BCL6 Intron 9, and TARS. Enrichment of DNA bound to BCL6 was calculated relative to the signal obtained from the $\beta$-actin antibody, using the $2^{-\mathrm{DCt}}$ method.

\begin{tabular}{|l|l|l|}
\hline & Forward & Reverse \\
\hline BCL6 Exon 1 & $\begin{array}{l}\text { 5'- } \\
\text { GCAGTGGTAAAGTCCGAA } \\
\text { GC }\end{array}$ & 5'- \\
& AGCAACAGCAATAATCACCTG \\
\hline BCL6 Intron & 5'- & \\
\hline TARS & ATT' & 5'-AATTTGCCCAAAACATGGTT \\
& $\begin{array}{l}\text { TATCTACGGTGTCCGGGA } \\
\text { AG }\end{array}$ & \\
\hline
\end{tabular}

\section{Results}

BCL6 expression was analysed in glioma using publically available microarray data (Oncomine, Rhodes et al 2007). BCL6 transcript was higher in glioblastoma than in normal brain in several independent studies (Fig 1A), and the level of transcript increased with grade (data not shown). BCL6 protein expression was examined by immunohistochemistry in $>60$ GBM tumor specimens (Fig 1B). Tumor sections examined had an average of $10 \%$ BCL6 positive cells, although the number ranged from $0-45 \%$ (Fig 1C). In many sections the BCL6+ cells appeared to be perivascular, so the variation in BCL6+ cells between tumors may represent a coincidental difference in the vascularity of sections sampled. Next, the p53 and MGMT status of each tumor was determined and correlated to BCL6 expression (Fig 1C). The proportion of each tumor that was BCL6 positive was similar 
regardless of whether the tumor had wild-type or mutant p53. Intriguingly, MGMT expressing tumors had a higher proportion of BCL6-positive cells.

Next, BCL6 expression in a panel of glioblastoma cell lines was examined by immunofluorescence microscopy (Fig 1D and data not shown). BCL6 protein level varied - the LN18 cell line had substantial nuclear expression in most cells, while the primary tumour-derived line NZG-0906 had strong expression in a proportion of cells, and NZG-1003 had very low, but detectable nuclear expression in the majority of cells. Other glioblastoma lines, including U87-MG and T98G, also expressed nuclear BCL6 protein (data not shown).

To assess the effect of DNA damaging therapy on BCL6, the cell line panel were treated with temozolomide, ionizing radiation and doxorubicin, then BCL6 protein level and sub-cellular localization assessed by both western blot and immunofluorescence. Consistent with Fig 1D, LN18 cells had a low but generally detectable basal level of BCL6 expression (Fig 2A). Cells were treated with multiple doses of a physiologically achievable concentration of temozolomide $(10 \mathrm{mM})$ over 2 weeks. This led to significant BCL6 induction in all cells tested - LN18 (Fig 2A), T98G (Fig 2B), NZG-1003 and NZG-0906 (data not shown). Similarly, cells were exposed to 5 daily fractions of $2 \mathrm{~Gy}$ ionizing radiation, or one dose of $10 \mathrm{~Gy}$. Both of these induced BCL6 expression in all lines tested - LN18, T98G, NZG-0906, and NZG-1003 (Fig 2 and data not shown). The effect of doxorubicin was also determined, because although does not cross the blood-brain barrier in vivo it has demonstrable efficacy against glioblastoma in vitro [19]. All cell lines tested upregulated BCL6 with doxorubicin exposure, similar to temozolomide and ionising radiation. At very low dose, down to $15 \mathrm{nM}$, doxorubicin had little direct impact on viability of glioblastoma cell lines, but led to significant induction of BCL6 over 24 hours. The doxorubicin effect on BCL6 level increased with dose and was sustained up to 72 hours. Immunofluorescence of dox-treated cells confirmed that induced BCL6 was predominantly nuclear, (Fig 2C), suggesting that it should be transcriptionally active.

The effect of therapy on BCL6 expression was assessed in vivo using a murine intracranial model. Consistent with all the human cell lines, BCL6 protein was up- 
regulated by doxorubicin, radiation and temozolomide treatment in vitro (data not shown). GL261 cells were implanted intra-cranially into C57/BL6 mice, and once significant tumor burden was established by the onset of weight loss, 7 Gy of whole brain irradiation was delivered [20] then tissue collected 24 or 48 hours after irradiation. BCL6 protein was analysed by immunofluorescence in the brain tumor, and compared to normal brain in the opposite hemisphere (Fig 2D). Localisation of tumor was determined by DAPI-staining of nuclei, which showed the densely packed irregular nuclei typical of this model [19]. There was a low level of basal BCL6 expression in the tumors, which was higher $24 \mathrm{~h}$ post-irradiation, and prominent by 48 hours. No BCL6 was observed in the normal brain tissue, either before or after irradiation.

The transcriptional activity of BCL6 in glioblastoma was determined using a BCL6luciferase reporter construct (BCL6 ${ }_{4}$-tk-LUC) [25]. In order to determine the effect of BCL6 on transcription in glioblastoma, each reporter construct was cotransfected into either LN18 or U87-MG cells with wild-type BCL6. Western blotting showed that BCL6 was strongly over-expressed upon transfection (Fig 3A). There was consistently a slight negative effect on cell viability from over-expression of BCL6 (data not shown), so in order to control for this effect every transfection was imaged and relative cell number determined before cell lysis. Luciferase activity was measured in cell lysate, and normalized back to cell number. The level of luciferase was then compared to cells transfected with the respective reporter construct alone. These data clearly showed that over-expressed BCL6 specifically repressed transcription from the BCL6 reporter, indicating that the BCL6 repression pathway was intact and functional in the glioblastoma cell lines.

Given that doxorubicin and ionizing radiation both induced BCL6 protein, LN18 cells were transfected with either the BCL6 or control reporter plasmid, then treated with either doxorubicin or ionizing radiation to determine whether induced BCL6 had transcriptional repressor activity, similar to over-expressed BCL6. Three different doses of doxorubicin - 50, 100 and $200 \mathrm{nM}$ - were used for 48 hours prior to imaging, cell quantitation and lysis. Luciferase activity from the control plasmid decreased by $50 \%$ in response to doxorubicin, suggesting a general negative effect of doxorubicin, either on transcription, expression or activity of the luciferase 
enzyme. In contrast, luciferase activity from the BCL6 reporter increased, doubling relative to the untreated control (Fig 3C). When compared to the effect of dox on the control plasmid, the activation was even greater. This apparent activation of transcription was unexpected, given the transcriptional repression activity of BCL6 observed upon over-expression. In confirmation, an even larger increase was observed in cells treated with 10Gy of ionizing radiation - there was little or no effect of radiation on the control reporter, but activity from the BCL6 reporter increased by more than 300\% (Fig 3D). These data strongly suggested that therapy-induced BCL6 acted as an activator of transcription, rather than a repressor. Similar data were obtained from the U87-MG cell line (data not shown).

Transcriptional repression by BCL6 generally requires the co-repressor proteins BCOR, NCOR1 and SMRT (NCOR2), so co-repressor expression was examined by quantitative RT-PCR (Fig 4A) and Western blot (Fig 4B). Each co-repressor was expressed in untreated LN18 and U87-MG cells, and transcription of each was not altered by treatment with either doxorubicin or ionising radiation. Interestingly, doxorubicin treatment reproducibly dropped the protein level of each co-repressor in LN18 cells, as well as that of alpha tubulin, used as a loading control. Dox also modestly reduced co-repressor levels in U87-MG cells, but had little to no effect on beta-actin expression. Ionising radiation treatment did not affect co-repressor levels in either cell line. The loss of co-repressor protein was consistent with the lack of transcriptional repression by induced BCL6 in doxorubicin-treated cells.

We wanted to determine whether dox-induced BCL6 had the same DNA binding ability as over-expressed BCL6. An electrophoretic mobility shift assay was performed, using the BCL6 consensus binding site TTCCTAGAA. LN18 cells were transfected with wild-type BCL6 or GFP, then nuclear extracts isolated after 48 hours and incubated with biotin-labeled DNA probe, before electrophoresis of the probe-protein complex and detection with streptavidin-HRP. Three specific DNA-bound complexes containing over-expressed BCL6 were identified by competition with unlabeled probe (a, b and c, Fig 5A). Supershift analysis with specific antibodies demonstrated that endogenous co-repressors BCOR, NCOR1 and SMRT were in complex with BCL6, consistent with robust repression by overexpressed BCL6 observed in the luciferase assay. Cells were then incubated with 
doxorubicin concentration used in the reporter assay, before nuclear extracts were collected for analysis. However, no binding to the BCL6 consensus binding site could be observed, either with endogenous or with doxorubicin-induced BCL6 (Fig $5 B)$.

To determine whether endogenous BCL6 could bind DNA at all, chromatin immunoprecipitation was carried out in LN18 cells. Initially, the ability of endogenous BCL6 to bind to a positive control sequence was examined. Interaction was observed between BCL6 and exon 1 of the BCL6 gene, and not to the negative control region of BCL6 intron 9 (Fig 5C). The strength of the interaction varied somewhat but was consistently detected above background. BCL6 was also found on a region of the TARS gene, another known BCL6 interacting genomic region[26], confirming that endogenous BCL6 could bind DNA in an appropriate context, although not always at a high level. Doxorubicin treatment did not significantly change BCL6 binding at either the BCL6 or TARS loci, as assessed at 8 and 48 hours post-treatment. This suggested that DNA binding by BCL6 did not require the presence of the co-repressors, consistent with the lack of direct transcriptional repression.

In other cell types, BCL6 activity is dependent on co-repressor interaction with the lateral groove of the BTB domain. Given the lack of repression, and of corepressor expression in glioblastoma after doxorubicin treatment, we used two inhibitors that specifically block protein interactions at the BTB domain - the peptide mimetic RI-BPI and the small molecule FX1 - and compared them to a general loss of BCL6 activity, by siRNA and a dominant negative BCL6 mutant.

The effect of BCL6 reduction by siRNA was determined in three different lines LN18, NZG-1003, and T98G, and the effect on apoptosis quantified by analysis of active caspase-3 (Figure 6A). Consistent with poor apoptosis in glioblastoma, the total number of cells with active caspase-3 was low. However, apoptotic cells increased from control siRNA to BCL6-siRNA treated cells, 2-fold for NZG-1003 and T98G, and 7-fold in LN18. The efficacy of siRNA knockdown was measured by flow cytometry for intracellular BCL6 protein. Knock-down of BCL6 was not highly efficient but within the apoptotic population, BCL6 expression decreased 
(Fig 6A, right panel), consistent with a protective effect of BCL6 expression in glioblastoma.

As a comparison, BCL6 was inhibited using the peptide mimetic inhibitor RI-BPI, which specifically blocks BCL6 co-repressor interaction by binding in the lateral groove of the BTB domain, a major protein interaction domain [27]. Both NZG1003 and LN18 cell lines were treated with $2 \mathrm{mM}$ RI-BPI for 24 hours, then apoptosis quantified by annexin- $\mathrm{V}$ positivity. Consistent with the caspase- 3 data, an annexin-V positive apoptotic phenotype emerged with BCL6 inhibition (Figure 6B). Again, only a small population of cells were involved. In LN18 cells, anxV+/PIcells increased 10 -fold, from $0.2 \%$ to $2.1 \%$, while the anxv $+/ \mathrm{PI}+$ increased from $0.02 \%$ to $0.9 \%$. This led to a 10 -fold increase in the total anxV+ apoptotic cells, from $0.2 \%$ to $3 \%$. In NZG-1003, total anxV+ cells increased by $50 \%$ with BCL6 inhibition, from 12.4 to $17.9 \%$. There was a 2.5 -fold increase in an $\mathrm{VV}+/ \mathrm{PI}$ negative cells, from $1.9 \%$ to $4.8 \%$, as well as increased anxV+/PI+ population. Again, the cell numbers were low but the data were reproducible across 3-5 independent experiments.

The effect of total BCL6 reduction was equivalent to specifically blocking proteinprotein interaction at the $\mathrm{BTB}$ pocket, suggesting that the BTB pocket is a key mediator of BCL6 activity. This was not sufficient to induce widespread apoptosis. However, the induction of apoptosis per se is not critical for effective therapy - as long as cells die, the actual mechanism is arguably unimportant.

To look at "all death" after short-term loss of BCL6 activity, we simply used propidium iodide uptake. Three cell lines were transfected with DN-BCL6-GFP, a BCL6 construct with intact BTB dimerisation domain, but a deletion in the zinc finger domain. This dimerizes with and inhibits endogenous BCL6 binding DNA, hence blocking transcription activity of the endogenous protein. GFP expression (Figure 7A) allowed identification and purification of transfected cells. GFP + cells were gated and the effect of DN-BCL6-GFP on viability determined as the proportion of PI+ GFP+ cells (Figure 7B), compared to the effect of GFP alone. GFP expression had a negligible effect in LN18 cells, but was detrimental to NZG0906 and NZG-1003, with 9-16\% of GFP+ cells PI+ after 24hrs. In LN18 and 
NZG-0906, DN-BCL6-GFP expression doubled the proportion of PI+ cells, from $2-4 \%$ and from $16-31 \%$ respectively. In NZG-1003, DN-BCL6-GFP caused a 4fold increase in non-viable cells, from 9-36\%. In hypoxic conditions, hypoxia alone increased PI+ cells, but inhibition of BCL6 by DN-BCL6-GFP still increased the number of non-viable cells 2.5 times, from $4 \%$ to $10 \%$ (Fig $7 \mathrm{~A}$, right panel).

The proportion of non-viable cells after BCL6 inhibition was reproducible but small. To determine the long-term consequence of BCL6 inhibition, transfected DN-BCL6-GFP+ cells were sorted from the non-transfected GFP-cells, and the clonogenic potential of each sub-population compared (Figure 7B), again with GFP+ cells as a control. Relative plating efficiency, or the number of colonies formed from a specific number of sorted GFP+ cells was determined by comparison to colonies from the same number of sorted GFP- cells, was determined for DN-BCL6-GFP and GFP alone. GFP alone had a detrimental effect on LN18 cells, with a 30\% reduction in plating efficiency. However, DN-BCL6GFP doubled the effect, with a $60 \%$ reduction. In T98G there was no effect of GFP expression on long-term survival, and greater than $50 \%$ reduction in plating efficiency with DN-BCL6-GFP, demonstrating clearly that loss of BCL6 activity was sufficient to reduce viability. Similar results were seen with the other cell lines NZG1003 and NZG0906 (data not shown).

To compare loss of overall activity, to a specific interruption of the BTB protein interaction domain, clonogenicity was determined using the small molecule BCL6 inhibitor FX1 [22], which also binds the lateral groove. The IC50 for FX1 on LN18, NZG-1003 and NZG-0906 was determined, and shown to be 25-40 uM, similar to lymphoma cell lines (data not shown and [22]). LN18 cells were treated with either 25 or $40 \mathrm{uM}$ for 24 hours, then the relative plating efficiency compared between treated and untreated cells. At $40 \mathrm{uM}$, BCL6 inhibition decreased clonogenicity completely, so that no colonies were formed (data not shown). At $25 \mathrm{uM}$ there was a $50-70 \%$ reduction in colony formation across all cell lines (Fig 7C).

\section{Discussion}

In this study, we assessed the potential role of the BCL6 protein in glioblastoma survival and therapy resistance. We directly demonstrated a key role for BCL6 in 
survival of glioblastoma cells in vitro. Differential expression analysis showed that BCL6 transcript level is higher in GBM than in normal brain. These findings are consistent with the recent literature, with reported BCL6 overexpression in many cancer cells compared to normal tissue, including gall bladder, breast, and colorectal cancer $[6,7,9]$. That BCL6 may have been localized to perivascular regions of glioblastoma is particularly interesting. If this is confirmed upon further analysis it might suggest BCL6 is expressed in cancer stem cells that inhabit the perivascular niche [28]. Co-staining BCL6 with GBM cancer stem cell markers such as CD133, integrin a6, notch or IL8 receptors [29, 30] would support this, and this is currently under investigation.

Previous publications to link glioma and BCL6 [12] saw a correlation between BCL6 and p53, predominantly in IDH1+ glioblastoma, a subset of secondary tumors arising from lower grade glioma. Our collection was predominantly primary glioblastoma, but did include some secondary disease. We did not see any correlation between p53 status and BCL6 expression. However, an interesting correlation was observed between BCL6 and MGMT expression, with higher BCL6 expression observed in MGMT positive tumours. Recent data show MGMT expression is commonly expressed by macrophages, and some tumours classified as MGMT+ actually reflect high levels of MGMT+ macrophage infiltration [31]. The observed association between MGMT and BCL6 could therefore simply mean that BCL6 is higher in tumours with greater macrophage infiltration. The relevance of this is not yet clear - BCL6 expression in glioblastoma cells in vitro is clearly not in macrophages, and more investigation is required.

Consistent with literature in other cell types[32], genotoxic stress induced by the standard glioblastoma therapy temozolomide and ionizing radiation, induced more BCL6 expression in glioblastoma, which was nuclear-localized. The induced protein could bind DNA, as observed by chromatin immunoprecipitation, and activate expression from a reporter gene. The nature of the DNA sequence recognized by BCL6 in glioblastoma, and how it interacts with the transcriptional machinery in the cell, is not yet clear. A single copy of a known consensus binding site was not enough to bind endogenous BCL6, but 4 sites multimerised in front of a reporter could direct BCL6-mediated activity, and BCL6 could bind genomic 
DNA, as determined by chromatin immunoprecipitation, so the context is clearly important, and more work will be required to determine the requirements for BCL6 binding in glioblastoma. Clearly, the DNA sequence

context is important, and more work will be required to determine the requirements for BCL6 binding in

glioblastoma, and the identity of the BCL6 target genes.

More curious is the apparent transcriptional activation activity of BCL6 in glioblastoma. It is not unheard of for a transcription factor to switch from repression to activation - PLZF, a BTB/ZF protein closely related to BCL6 can be converted from a repressor to an activator in different cell contexts, by deacetylation followed by phosphorylation [33]. Whether a similar mechanism has occurred for BCL6 is glioblastoma is now under investigation. Interestingly, a recent publication also supports the hypothesis that BCL6 has become an activator in glioma - while not explicitly describing the mechanism or proving it was a direct effect, an association was seen between BCL6 and increased expression of the Axl tyrosine kinase [13].

We tested the hypothesis that BCL6 drives apoptosis resistance in glioblastoma, and loss of BCL6 would allow apoptosis to occur. The apoptotic effect of BCL6 loss was consistent but not strong, either with siRNA or with inhibitors. While BCL6 may have a small role in preventing apoptosis, supported by the previously observed correlation between apoptosis and BCL6 expression[12], we concluded that BCL6 activity is not largely responsible for poor apoptosis in glioblastoma. Our data are the first direct measure of the effect of BCL6 inhibition on apoptosis in glioblastoma, Taking an agnostic approach to cell death, measuring just loss of viability and long term proliferative potential. This clearly demonstrated that BCL6 is very important in glioblastoma survival- BCL6 inhibition using the small molecule FX1 at $40 \mathrm{uM}$ stopped all

long-term proliferation, which decreased significantly at $25 \mathrm{uM}$. This is supported by the recent finding that BCL6 inhibition stopped proliferation [13], although contrary to Xu et al. we can see no evidence of senescence in any of our BCL6inhibited cells. 
In order to confirm the importance of BCL6 for glioblastoma, and to begin to understand the mechanism of action, we made multiple attempts to knock out the BCL6 locus using nuclease-mediated genome editing. These were all unsuccessful while the BCL6 locus could be edited in other cell types, and other loci edited in glioblastoma cells, any glioblastoma cell that had nuclease editing of the BCL6 locus died within 48 hours and could not be selected (data not shown). These data strengthened our hypothesis that BCL6 is essential for survival of glioblastoma cells in vitro.

Evidence for a role of BCL6 in survival of people with glioblastoma would be important for translation of BCL6 inhibition to the clinic. We could not find proof that BCL6 was associated with a worse outcome in our cohort of tumour samples. However, analysis of copy number and survival using the Rembrandt database [34] provided a hint of support. Three GBM patients had loss of 3q27, including the BCL6 locus, and increased survival (data not shown). While three is not a significant sample, this supports our hypothesis that BCL6 plays an important role in survival of glioblastoma in vivo. Further, a recent publication has suggested that expression of BCL6 protein was associated with worse survival in a cohort of glioblastoma patients [13].

These data strongly suggest BCL6 is a genuine target for therapy in glioblastoma. People diagnosed with glioblastoma have few options for treatment, and those options are not effective. BCL6 inhibition has been shown to be highly effective in animal models of lymphoma and leukemia, and inhibitors are currently in clinical development. BCL6 inhibition in glioblastoma is highly feasible - several inhibitors, both peptide and small molecule, have been developed for BCL6 in lymphoma and leukemia $[4,27]$, and some have been identified that pass through the blood brain barrier and accumulate in the brain (unpublished data). In glioblastoma, BCL6 inhibition presents a highly tumor-specific target and has real potential to improve the outcome for people with this disease. 
Acknowledgements. We would like to thank Maria Celina Capistrano and Mathew Storey for their technical assistance. Financial support came from the Cancer Society of New Zealand CSNZ13/25; Genesis Oncology Trust 1155-RPG and 1457-RPG, and Victoria University of Wellington, to MJM. Cancer Society of New Zealand, Wellington Division, CT Collins PhD scholarship to NMJ. The funding bodies had no role in study design, analysis or interpretation of data, writing of the report or the decision to submit the manuscript for publication. 


\section{References}

1. Stupp, R., et al., Effects of radiotherapy with concomitant and adjuvant temozolomide versus radiotherapy alone on survival in glioblastoma in a randomised phase III study: 5-year analysis of the EORTC-NCIC trial. Lancet Oncol, 2009. 10(5): p. 459-66.

2. Stegh, A.H., et al., What drives intense apoptosis resistance and propensity for necrosis in glioblastoma? A role for Bcl2L12 as a multifunctional cell death regulator. Cell Cycle, 2008. 7(18): p. 2833-9.

3. Xu, Y.Y., et al., Development of targeted therapies in treatment of glioblastoma. Cancer Biol Med, 2015. 12(3): p. 223-37.

4. Parekh, S., G. Prive, and A. Melnick, Therapeutic targeting of the BCL6 oncogene for diffuse large B-cell lymphomas. Leuk Lymphoma, 2008. 49(5): p. 87482.

5. Cerchietti, L.C., et al., A small-molecule inhibitor of BCL6 kills DLBCL cells in vitro and in vivo. Cancer Cell, 2010. 17(4): p. 400-11.

6. Ribeiro, I.P., et al., Genetic gains and losses in oral squamous cell carcinoma: impact on clinical management. Cell Oncol (Dordr), 2014. 37(1): p. 2939.

7. Sena, P., et al., Morphological and quantitative analysis of BCL6 expression in human colorectal carcinogenesis. Oncol Rep, 2014. 31(1): p. 103-10.

8. Liang, P.I., et al., BCL6 overexpression is associated with decreased p19 ARF expression and confers an independent prognosticator in gallbladder carcinoma. Tumour Biol, 2014. 35(2): p. 1417-26.

9. Walker, S.R., et al., The transcriptional modulator BCL6 as a molecular target for breast cancer therapy. Oncogene, 2015. 34(9): p. 1073-82.

10. Tiberi, L., et al., A BCL6/BCOR/SIRT1 complex triggers neurogenesis and suppresses medulloblastoma by repressing Sonic Hedgehog signaling. Cancer Cell, 2014. 26(6): p. 797-812.

11. Chen, Y.W., et al., High BCL6 expression predicts better prognosis, independent of BCL6 translocation status, translocation partner, or BCL6deregulating mutations, in gastric lymphoma. Blood, 2006. 108(7): p. 2373-83.

12. Ruggieri, S., et al., Translocation of the proto-oncogene Bcl-6 in human glioblastoma multiforme. Cancer Lett, 2014. 353(1): p. 41-51. 
13. Xu, L., et al., BCL6 promotes glioma and serves as a therapeutic target. Proc Natl Acad Sci U S A, 2017. 114(15): p. 3981-3986.

14. Kurosu, T., et al., BCL6 overexpression prevents increase in reactive oxygen species and inhibits apoptosis induced by chemotherapeutic reagents in B-cell lymphoma cells. Oncogene, 2003. 22(29): p. 4459-68.

15. Duy, C., et al., BCL6 enables Ph+ acute lymphoblastic leukaemia cells to survive BCR-ABL1 kinase inhibition. Nature, 2011. 473(7347): p. 384-8.

16. Cerchietti, L.C., et al., BCL6 repression of EP300 in human diffuse large B cell lymphoma cells provides a basis for rational combinatorial therapy. J Clin Invest, 2010. 120(12): p. 4569-82.

17. Patel, V.N., et al., Network signatures of survival in glioblastoma multiforme. PLoS Comput Biol, 2013. 9(9): p. e1003237.

18. Authier, A., et al., Enhanced immunosuppression by therapy-exposed glioblastoma multiforme tumor cells. Int J Cancer, 2015. 136(11): p. 2566-78.

19. Broadley, K.W., et al., Side population is not necessary or sufficient for a cancer stem cell phenotype in glioblastoma multiforme. Stem Cells, 2011. 29(3): p. $452-61$.

20. Grasso, C., et al., Pharmacological doses of daily ascorbate protect tumors from radiation damage after a single dose of radiation in an intracranial mouse glioma model. Front Oncol, 2014. 4: p. 356.

21. Cerchietti, L.C., et al., A peptomimetic inhibitor of BCL6 with potent antilymphoma effects in vitro and in vivo. Blood, 2009. 113(15): p. 3397-405.

22. Cardenas, M.G., et al., Rationally designed BCL6 inhibitors target activated B cell diffuse large B cell lymphoma. J Clin Invest, 2016. 126(9): p. 3351-62.

23. Andrews, N.C. and D.V. Faller, A rapid micropreparation technique for extraction of DNA-binding proteins from limiting numbers of mammalian cells. Nucleic Acids Res, 1991. 19(9): p. 2499.

24. Wegner, W., G. Burckhardt, and M. Henjakovic, Transcriptional regulation of human organic anion transporter 1 by B-cell CLL/lymphoma 6. Am J Physiol Renal Physiol, 2014. 307(11): p. F1283-91.

25. Chang, C.C., et al., BCL-6, a POZ/zinc-finger protein, is a sequence-specific transcriptional repressor. Proc Natl Acad Sci U S A, 1996. 93(14): p. 6947-52. 
26. Polo, J.M., et al., Transcriptional signature with differential expression of BCL6 target genes accurately identifies BCL6-dependent diffuse large B cell lymphomas. Proc Natl Acad Sci U S A, 2007. 104(9): p. 3207-12.

27. Polo, J.M., et al., Specific peptide interference reveals BCL6 transcriptional and oncogenic mechanisms in B-cell lymphoma cells. Nat Med, 2004. 10(12): p. 1329-35.

28. Pietras, A., et al., Osteopontin-CD44 signaling in the glioma perivascular niche enhances cancer stem cell phenotypes and promotes aggressive tumor growth. Cell Stem Cell, 2014. 14(3): p. 357-69.

29. Infanger, D.W., et al., Glioblastoma stem cells are regulated by interleukin-8 signaling in a tumoral perivascular niche. Cancer Res, 2013. 73(23): p. 7079-89.

30. Lathia, J.D., et al., Cancer stem cells in glioblastoma. Genes Dev, 2015. 29(12): p. 1203-17.

31. Hsu, C.Y., et al., Exclusion of histiocytes/endothelial cells and using endothelial cells as internal reference are crucial for interpretation of MGMT immunohistochemistry in glioblastoma. Am J Surg Pathol, 2013. 37(2): p. 264-71.

32. Phan, R.T., et al., Genotoxic stress regulates expression of the protooncogene Bcl6 in germinal center B cells. Nat Immunol, 2007. 8(10): p. 1132-9.

33. Xu, D., et al., Promyelocytic leukemia zinc finger protein regulates interferon-mediated innate immunity. Immunity, 2009. 30(6): p. 802-16.

34. Madhavan, S., et al., Rembrandt: helping personalized medicine become a reality through integrative translational research. Mol Cancer Res, 2009. 7(2): p. 15767. 


\section{Figure Legends:}

Figure 1. BCL6 is expressed in glioblastoma tumours and cell lines. A. Plots of BCL6 mRNA expression in normal brain tissue (1) compared to GBM tissue (2) in three independent representative studies (Sun, Lee and, Murat Brain respectively) from Oncomine (https://www.oncomine.org/resource/login.html). B. Representative IHC staining of $3 / 62$ human GBM tumours, highlighting heterogeneous BCL6 protein expression (brown staining). C. BCL6 expression level distribution in 62 human GBM tumours (top), and after stratification by p53 mutation status (middle) and MGMT expression (bottom). D. Immunofluorescent staining for BCL6 in glioblastoma cell lines LN18, NZG10/03 and NZG09/06. Immunofluorescence data are representative of at least 3 independent experiments, and are similar to other glioblastoma cell lines. Adjustments to brightness and contrast were used to improve visibility upon printing, and were applied to the whole image.

Figure 2. BCL6 expression was induced by DNA-damaging therapy in glioblastoma. A. LN18 cells were untreated (U), or treated with $10 \mathrm{mM}$ temozolomide every 2 days for 12 days ( 7 treatments total, upper left panel), 10 Gy of ionizing radiation (upper right) or increasing doses of doxorubicin as described (lower left). LN18 cells were treated with $1 \mathrm{mM}$ doxorubicin for 24-72 hours (lower right panel). BCL6 and alpha tubulin (TUBA) were detected by western blot $24 \mathrm{~h}$ after treatment, with Raji cell lysate used as a positive control for BCL6 expression. B. Cell line T98G was treated with increasing doses of doxorubicin, or $10 \mathrm{~Gy}$ ionizing radiation for $24 \mathrm{~h}$, and with $10 \mathrm{mM}$ TMZ every 2 days for 12 days (left panel.) NZG-1003, LN18 and NZG-0906 were treated with doxorubicin for 24 hours (centre panel), NZG-0906 was treated with 10 Gy ionizing radiation at 8, 16, 24 hours (right panel). BCL6 and alpha-tubulin were detected by western, and Raji used as a positive control. U, untreated cells. C. LN18 (left) and NZG-0906 (right) were grown on coverslips, left untreated (center panels) or treated with $3 \mathrm{mM}$ (LN18) or $1 \mathrm{uM}$ (NZG-0906) doxorubicin (lower panels), and BCL6 detected by immunofluorescence. An isotype antibody was used as a control on treated cells (upper panels). All data shown are representative of at least 3 independent 
experiments. Cropped images retain all bands, immunofluorescence was adjusted only for brightness and contrast over the entire image.

Figure 3. BCL6 is transcriptionally active in glioblastoma. A. Western blot for BCL6, alpha tubulin (TUBA), and beta actin (ACTB) of LN18 cells transfected with BCL6wt, GFP or non-transfected (N.T) with K562 and Raji cells as BCL6 negative and positive controls, respectively (left panel). Cells were harvested 48 hours after transfection. Luciferase assay of LN18 cells co-transfected with both BCL6 $6_{4}$-tkLUC and BCL6wt plasmids or PGL3 and BCL6wt plasmids and harvested 48 hours after transfection (right panel). The luciferase values were first normalized to cell number and then expressed as a proportion of their single transfected control (either BCL6 $4^{-}$ tkLUC or PGL3 alone). B. Luciferase assay of LN18 cells transfected with either BCL6 $_{4}$-tkLUC or PGL3 plasmid and treated with either 50, 100, or $200 \mathrm{nM}$ doxorubicin. C. Luciferase assay of LN18 cells transfected with either BCL6 $4^{-}$ tkLUC or PGL3 plasmid and treated with 10 Gy ionizing radiation. All assays were performed 48 hours after treatment.

Figure 4. BCL6 co-repressors are expressed in glioblastoma. A. q-RT-PCR of BCL6 co-repressors BCOR, NCOR1, and SMRT (NCOR2) in LN18 and U87-MG cells treated with $3 \mu \mathrm{M}$ doxorubicin, $10 \mathrm{~Gy}$ ionizing radiation, or untreated. The value 1/DCt is shown (x-axis), with the DCt value calculated against HPRT Ct. B. Western Blot for BCOR, NCOR1, SMRT (NCOR2), alpha tubulin (TUBA), and beta actin (ACTB) in LN18 and U87-MG cells treated with $3 \mu \mathrm{M}$ doxorubicin (Dox), 10 y ionizing radiation (IR), or untreated (Unt). All cells were harvested 48 hours after treatment.

Figure 5. Both over-expressed and drug-induced BCL6 can bind DNA. A. EMSA of LN18 nuclear lysate transfected with BCL6wt (lanes 2-7) or GFP (lanes 8 \& 9). Complexes were formed with nuclear lysate and biotin labelled probe alone (lanes 2 and 8), with unlabelled competitor DNA (lanes 3 and 9), with BCL6 antibody (B1) (lane 4), with BCOR antibody (B2) (lane 5), with NCOR antibody (N) (lane 6), and with SMRT antibody (S) (lane 7). a, b, c indicate complexes formed with BCL6 and co-repressors. B. EMSA of LN18 nuclear lysate treated with $0.05,0.1,0.2$ and $3 \mu \mathrm{M}$ doxorubicin and harvested 48 hours after treatment (lanes 2-6). C. Relative binding 
(fold change as compared to $\beta$-actin) of BCL6 in LN18 treated with $3 \mu \mathrm{M}$ doxorubicin and harvested 8 or 48 hours after treatment, or left untreated to BCL6 Exon 1, BCL6 Intron 9 or TARS. Fold change was calculated using the $2^{\text {-DCt }}$ method.

Figure 6. Both loss of BCL6 and blockade of lateral groove increased apoptosis. A. NZG-1003, LN18 and T98G cells were transfected with control (left panel) or BCL6 (center panel) siRNA, and cells with active Caspase 3 determined by flow cytometric analysis (y-axis). Caspase-3 positive apoptotic cell gate was established from cells stained with a control antibody, and the percentage of cells in the apoptotic gate is given. BCL6 level was measured in parallel by intracellular staining (x-axis). In the center panel, BCL6 level was determined in the apoptotic population, and the control siRNA (light grey) compared to the BCL6 siRNA (dark grey). The median fluorescence intensity for each population is shown, data are representative of at least 3 independent experiments. B. NZG-1003 and LN18 cells were treated with the BCL6 peptide mimetic inhibitor RI-BPI, or a vehicle control, for 24 hours and annexin V/PI staining used to identify apoptotic and nonviable cells. Proportion of cells is given for each quadrant. All data are representative of multiple independent experiments.

Figure 7. Both BCL6 inhibition by dominant negative BCL6 and blockade of lateral groove decreased viability and clonogenic potential. A. LN18, NZG-1003 and NZG-0906 were transfected with either a control GFP construct (upper panels) or a DN-BCL6-GFP construct (lower panels). After 24 hours, GFP positive cells were gated and propidium iodide positivity analysed by flow cytometry as a measure of non-viability. Histogram gates were established on unstained cells, and the number of cells given for each gate. Hypoxia - LN18 cells were grown in $0.5 \%$ oxygen for 24 hours, transfected with either GFP or DN-BCL6-GFP, and replaced into 0.5\% oxygen for 24 hours before analysis of GFP+ PI+ cells. B. GFP+ and GFP- cells were physically sorted from LN18 and T98G cells transfected with GFP, or DNBCL6-GFP, then clonogenic plating efficiency determined for GFP+ and GFPcells from each transfection. C. LN18 and T98G cells were treated with 25 uM FX1 for 24 hours, then clonogenic plating efficiency determined. All data shown are representative of at least 3 independent experiments. 


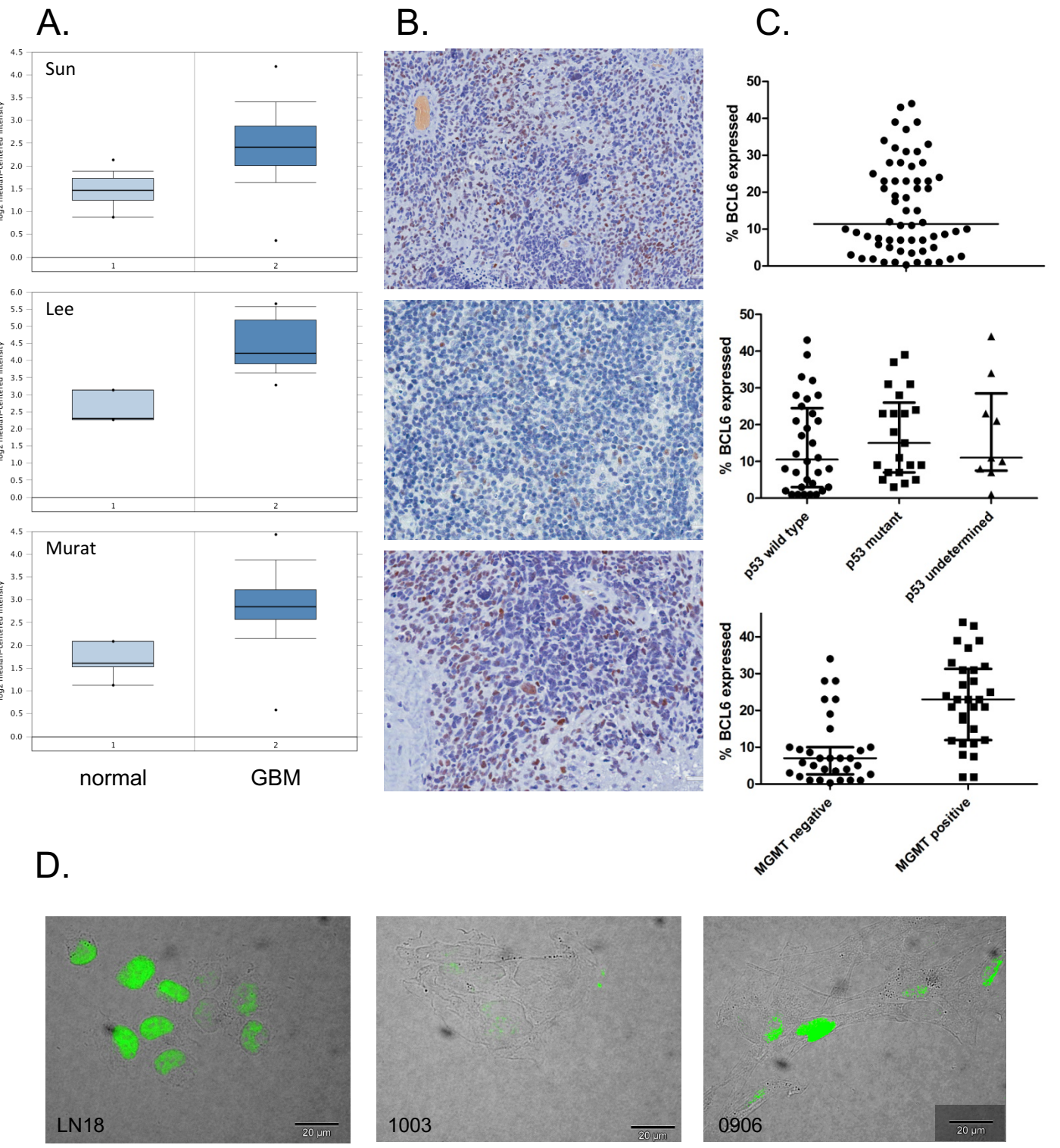

Figure 1 

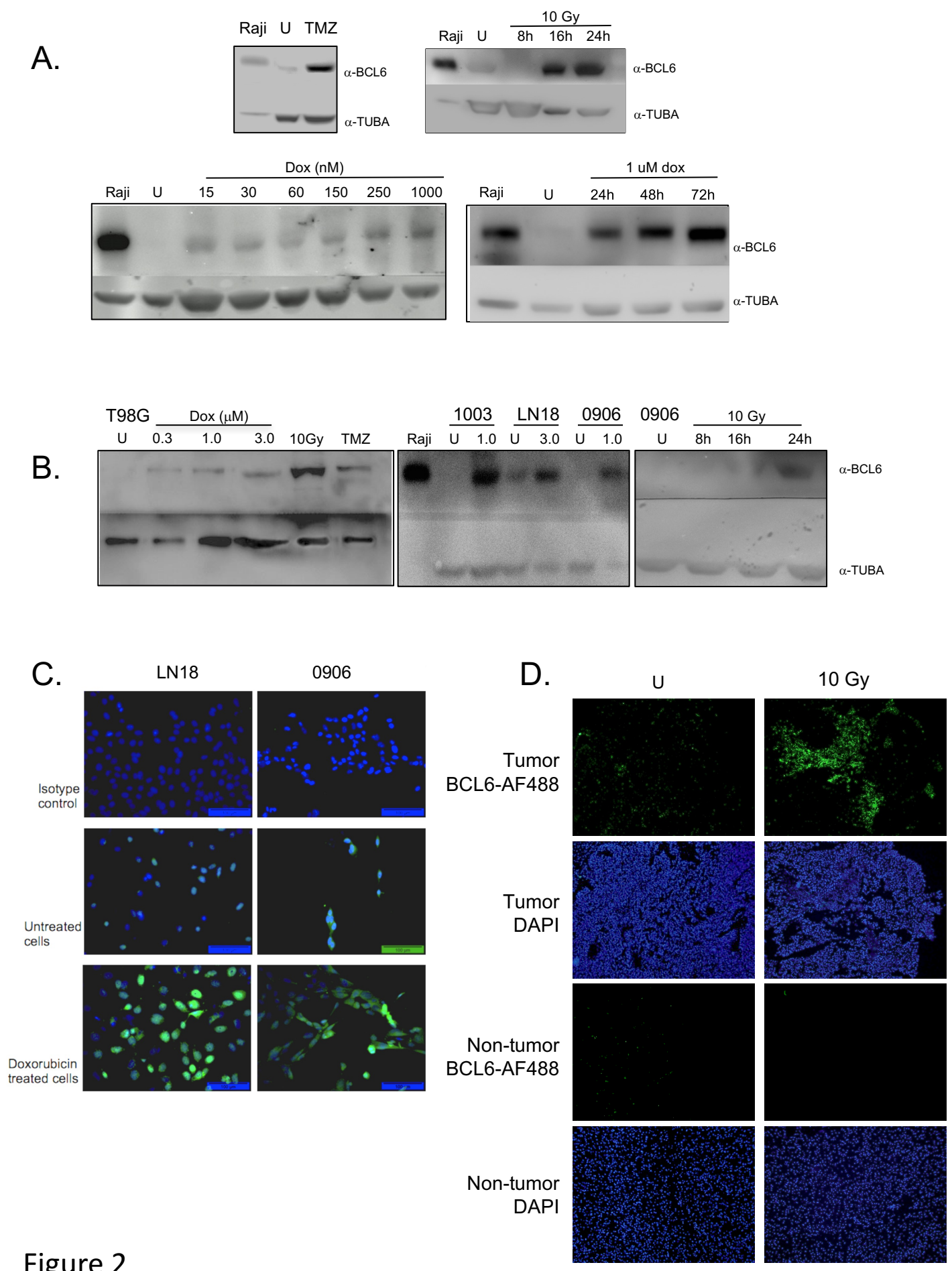

Figure 2 

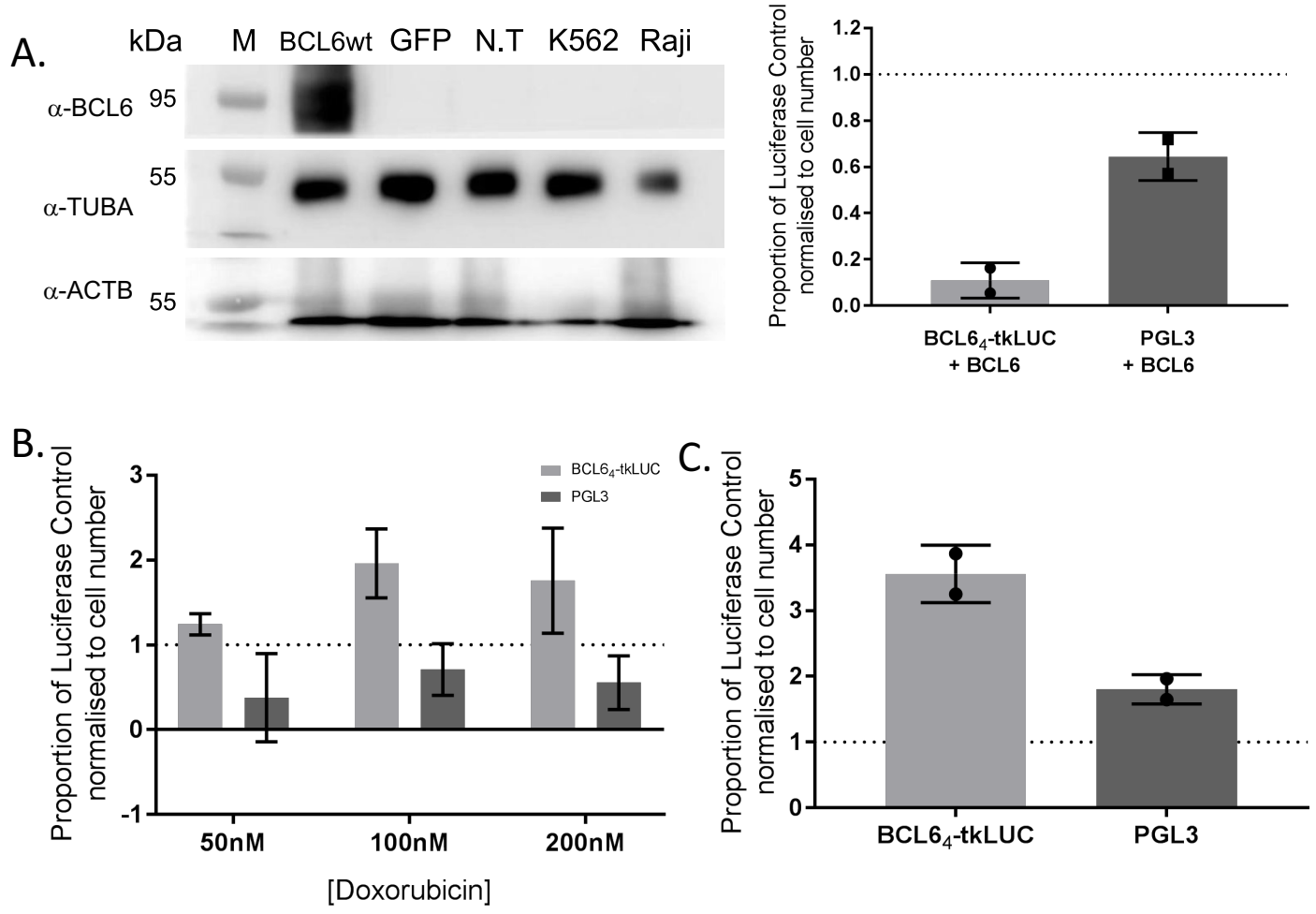

Figure 3 


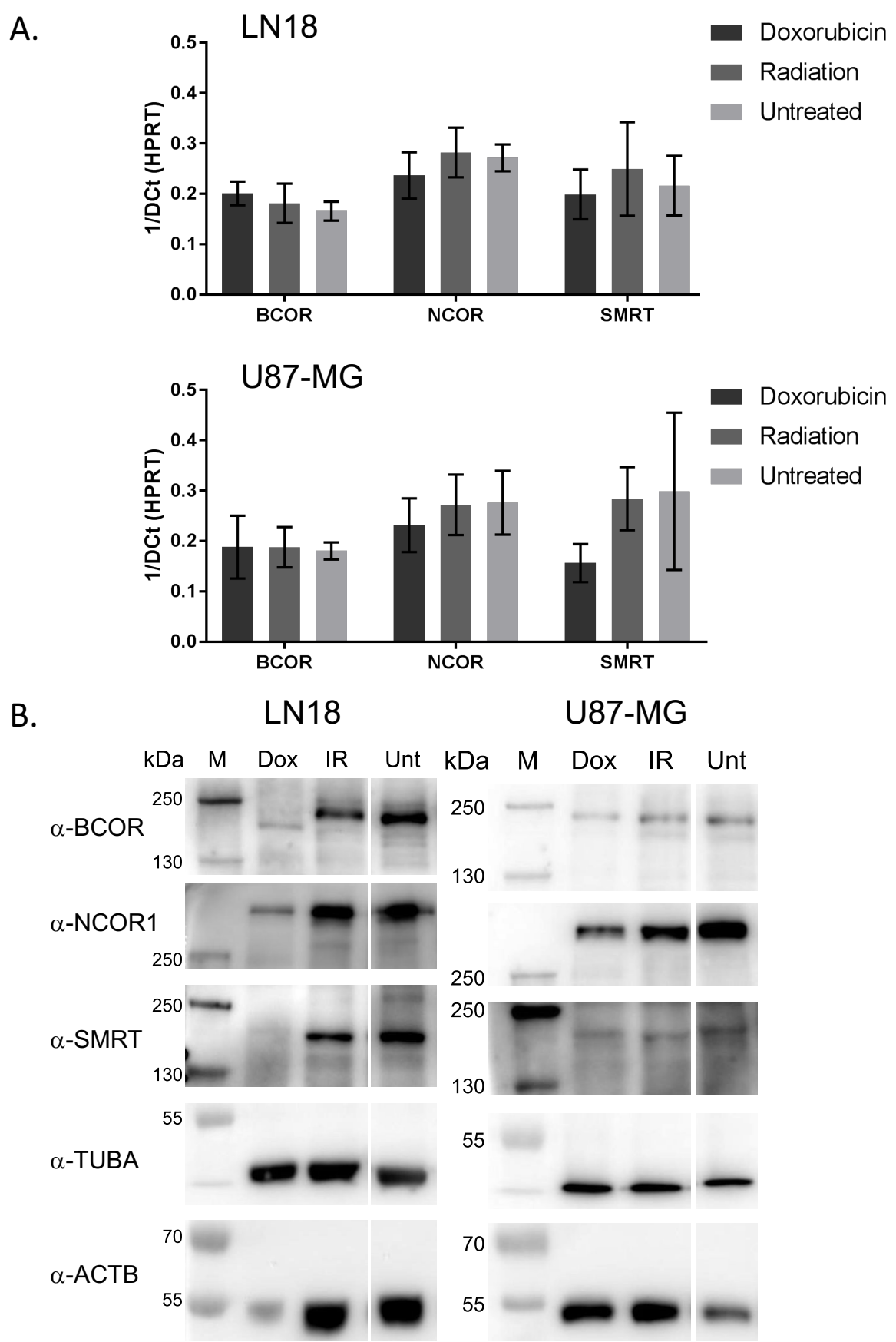

Figure 4 


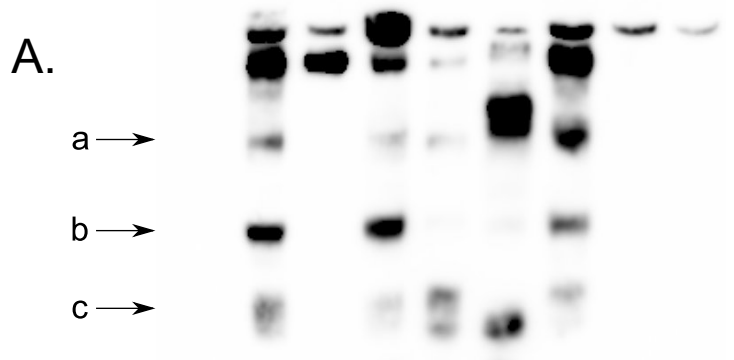

$\begin{array}{rccccccccc} & 1 & 2 & 3 & 4 & 5 & 6 & 7 & 8 & 9 \\ \text { BCL6 } & - & + & + & + & + & + & + & - & - \\ \text { GFP } & - & - & - & - & - & - & - & + & + \\ \text { Comp DNA } & - & - & + & - & - & - & - & - & + \\ \text { Antibody } & - & - & - & \text { B1 } & \text { B2 } & \text { N } & \text { S } & - & - \\ \text { Probe } & + & + & + & + & + & + & + & + & +\end{array}$

C.

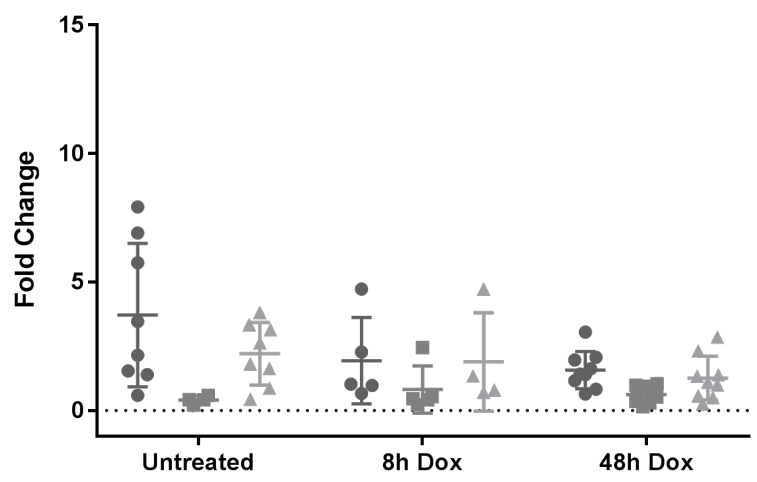

B.

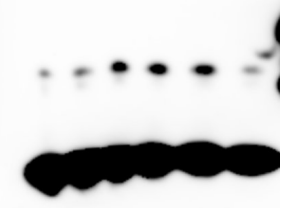

$$
\begin{array}{ccccccc} 
& 1 & 2 & 3 & 4 & 5 & 6 \\
\text { Untreated } & - & + & - & - & - & - \\
\text { Dox (uM) } & - & - & 0.05 & 0.1 & 0.2 & 3 \\
\text { Probe } & + & + & + & + & + & +
\end{array}
$$

Figure 5 

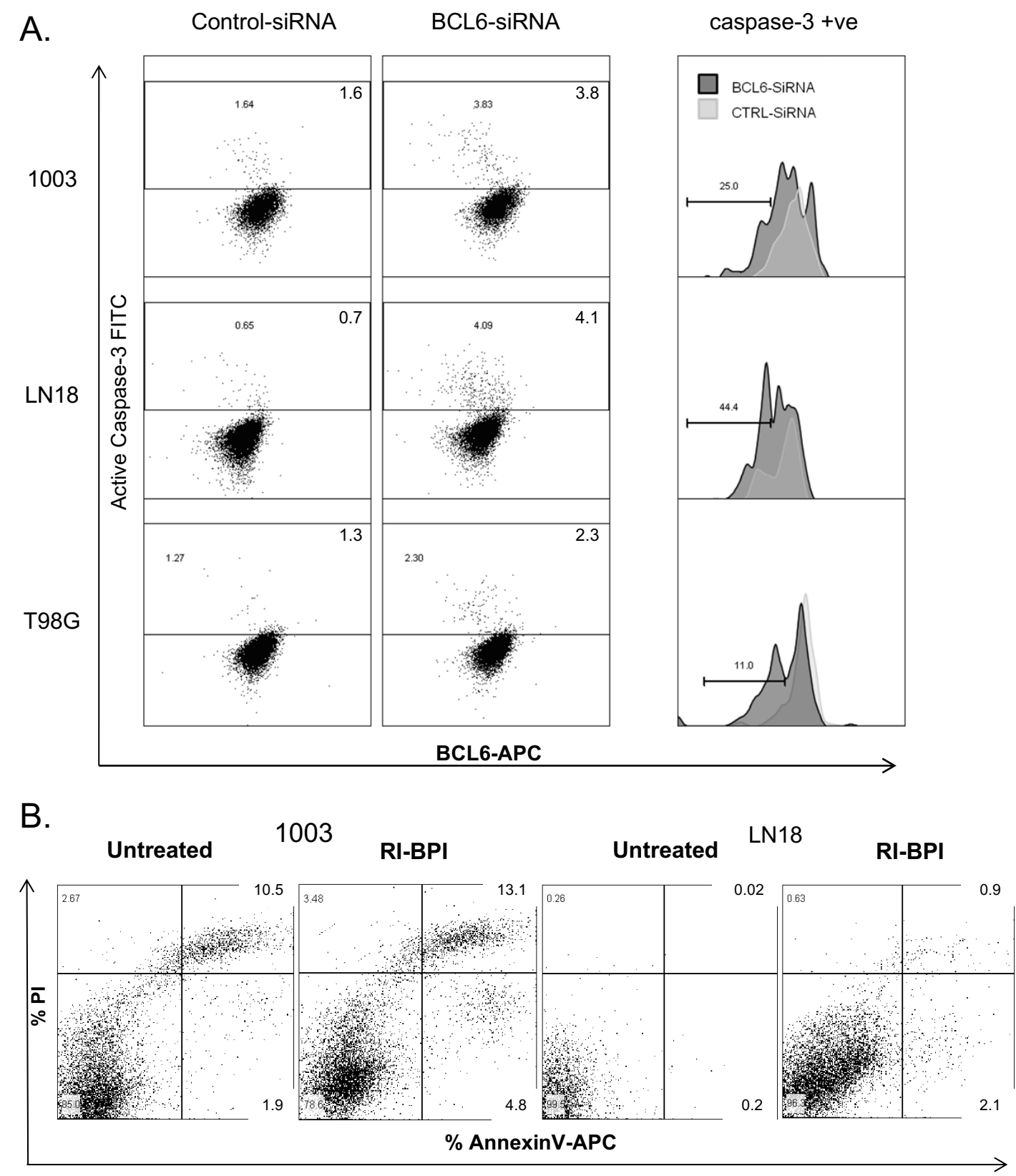

Figure 6 
A.

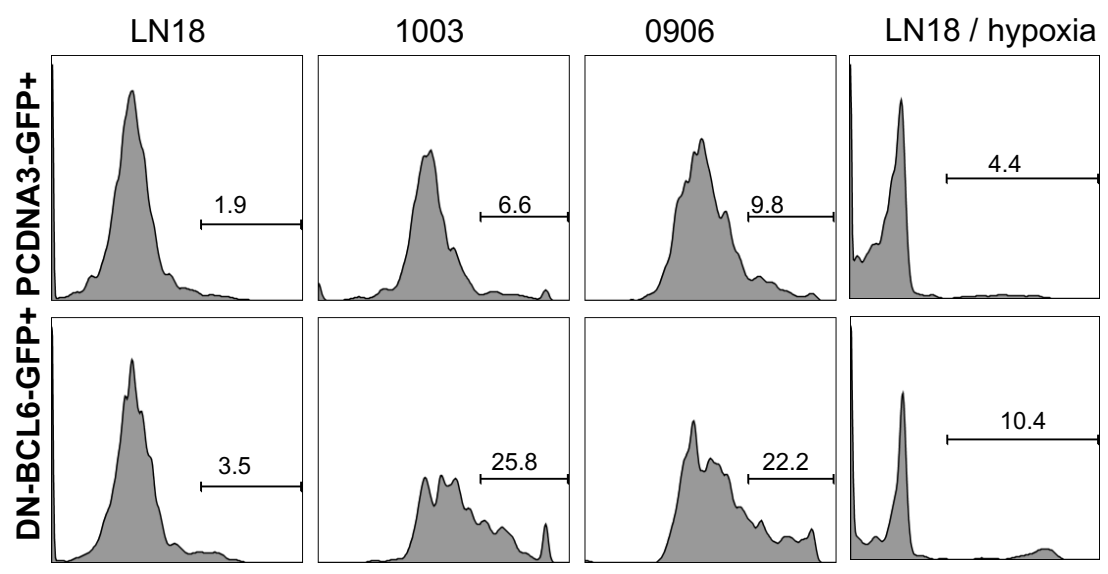

B.
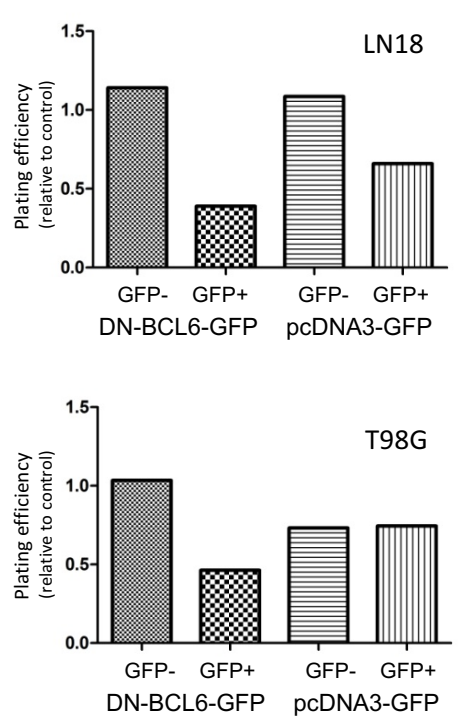

C.
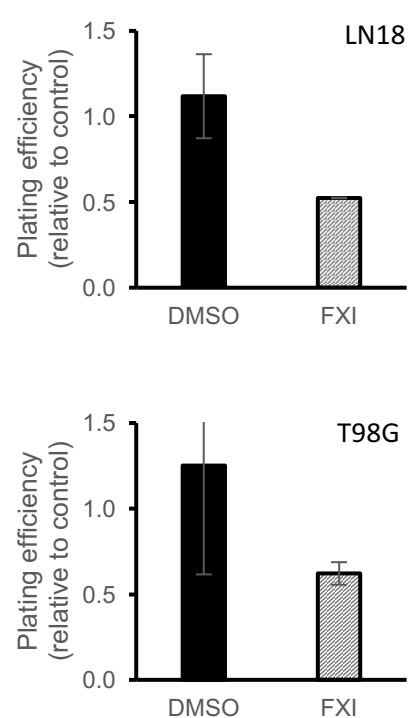

Figure 7 